University of Tennessee Health Science Center

UTHSC Digital Commons

$12-2016$

\title{
Endosomal Trafficking as a Determinant of Antifungal Tolerance in the Pathogenic Fungus Candida albicans
}

Arturo Luis Luna

University of Tennessee Health Science Center

Follow this and additional works at: https://dc.uthsc.edu/dissertations

Part of the Biological Phenomena, Cell Phenomena, and Immunity Commons, and the Medical Microbiology Commons

\section{Recommended Citation}

Luna, Arturo Luis (http://orcid.org/0000-0002-3129-4115), "Endosomal Trafficking as a Determinant of Antifungal Tolerance in the Pathogenic Fungus Candida albicans" (2016). Theses and Dissertations (ETD). Paper 417. http://dx.doi.org/10.21007/etd.cghs.2016.0417.

This Dissertation is brought to you for free and open access by the College of Graduate Health Sciences at UTHSC Digital Commons. It has been accepted for inclusion in Theses and Dissertations (ETD) by an authorized administrator of UTHSC Digital Commons. For more information, please contact jwelch30@uthsc.edu. 


\title{
Endosomal Trafficking as a Determinant of Antifungal Tolerance in the Pathogenic Fungus Candida albicans
}

\begin{abstract}
Several important antifungal drugs inhibit the synthesis of ergosterol, a lipid that modulates the thickness, fluidity and permeability of fungal cell membranes. These include the azole antifungals, which block ergosterol biosynthesis by inhibiting lanosterol demethylase (Erg11p). The resulting depletion of cellular ergosterol and the accumulation of 'toxic' sterol intermediates are both thought to cause plasma membrane dysfunction and ultimately growth arrest. However, the effects of ergosterol depletion upon the function of intracellular membranes and organelles are not well described. The purpose of this study was to characterize the effects of azole treatment upon the integrity of the Candida albicans vacuole, and determine if, in turn, vacuolar trafficking influences azole susceptibility. Profound fragmentation of the C. albicans vacuole was observed as an early consequence of azole treatment, before significant growth inhibition was observed. Using a genetic approach, we determined that vacuole fragmentation was a consequence of Erg11p inhibition rather than an off-target effect of azole treatment. Moreover, the degree of vacuole fragmentation following azole treatment was influenced by Erg3p, an enzyme in the ergosterol biosynthetic pathway involved in the production of toxic sterol intermediaries upon Erg11p inhibition. We also determined that vacuolar trafficking significantly impacts $C$. albicans susceptibility to azole antifungals and other ergosterol biosynthesis inhibitors. For instance, a vps $21 \Delta / \Delta$ mutant, blocked in membrane trafficking through the pre-vacuolar compartment (PVC), grew significantly more than wildtype controls in the presence of several azole antifungals under standard susceptibility testing conditions. Furthermore, the vps $21 \Delta / \Delta$ mutant was able to grow in the presence of the azoles despite depletion of cellular ergosterol. This phenotype resembles an exaggerated form of azole tolerance known as 'trailing growth', which has been described for some clinical isolates. In contrast, the vps $21 \Delta / \Delta$ mutant is hypersensitive to drugs that block alternate steps in ergosterol biosynthesis. The azole tolerance phenotype of the $C$. albicans vps $21 \Delta / \Delta$ mutant was independent of known azole resistance mechanisms such as the efflux pumps Cdr1p and Mdr1p. Moreover, the azole tolerance of the vps $21 \Delta / \Delta$ mutant was influenced by both $\mathrm{pH}$ and incubation temperature, consistent with trailing growth phenotypes. The $\mathrm{C}$. albicans vps $21 \Delta / \Delta$ mutant exhibits less plasma membrane permeabilization upon azole treatment, as determined by the release of a cytoplasmic luciferase reporter into the culture supernatant. Our results also reveal that the vps $21 \Delta / \Delta$ mutant has elevated levels of intracellular calcium and enhanced calcineurin activity, as evidenced by increased expression of a calcineurin responsive RTA2-GFP reporter construct in response to fluconazole. Furthermore, the azole tolerant phenotype of the vps $21 \Delta / \Delta$ mutant is dependent upon both calcium and calcineurin signaling. These findings underscore the importance of endosomal trafficking in determining the cellular consequences of azole treatment through modulation of intracellular calcium levels and calcineurin dependent responses. While we determined that deletion of VPS21 alone was not sufficient to confer a survival advantage upon C. albicans following azole treatment in the mouse model of vaginal candidiasis, the azole susceptibility of the vps $21 \Delta / \Delta$ mutant in the mouse model disseminated infection is yet to be tested. Moreover, it is unclear how, or if the azole tolerant phenotype of the vps $21 \Delta / \Delta$ mutant relates to that of trailing clinical isolates, or if these clinical isolates have abnormal endosomal trafficking.
\end{abstract}

\section{Document Type}

Dissertation

\section{Degree Name}

Doctor of Philosophy (PhD) 


\section{Program}

Biomedical Sciences

Research Advisor

Glen E. Palmer, Ph.D.

\section{Keywords}

Azoles, Candida albicans, Tolerance, Vacuolar Trafficking, Vps21p

\section{Subject Categories}

Biological Phenomena, Cell Phenomena, and Immunity | Medical Microbiology | Medical Sciences | Medicine and Health Sciences

\section{Comments}

One year embargo expires October 2017. 


\title{
Endosomal Trafficking as a Determinant of Antifungal Tolerance in the Pathogenic Fungus Candida albicans
}

\author{
A Dissertation \\ Presented for \\ The Graduate Studies Council \\ The University of Tennessee \\ Health Science Center
}

In Partial Fulfillment

Of the Requirements for the Degree

Doctor of Philosophy

From The University of Tennessee

By

Arturo Luis Luna

December 2016 
Portions of Chapter 2, 3, and 4 (C) 2015 by American Society for Microbiology. Portions of Chapter 4 and 6 (C) 2016 by American Society for Microbiology. All other material (C) 2016 by Arturo Luis Luna. All rights reserved. 


\section{DEDICATION}

To my parents, Luis and Virgilia, to my brothers and sister, for their love and support, and for encouraging me to pursue my goals. To my wife Kelly, for her love and for giving me the balance I need. 


\section{ACKNOWLEDGEMENTS}

My uttermost gratitude to my advisor, Glen Palmer, for all his teachings and lessons to make me a better scientist. The Spanish lessons may have to wait.

I would like to thank my committee members P. David Rogers, Brian M. Peters, Ben Kelly and David R. Nelson for their guidance.

Special thanks to the former and current members of the Palmer lab, Karen Eberle, Morgan Kerns, Hélène Tournu, Tracy Peters, Christian DeJarnette, and Arielle Butts, for their friendship, help and discussions. Also, I would like to thank Marjoleine Willems from the Peters Lab, as well as Andy Nishimoto, Jeffrey Rybak, Elizabeth Berkow and Sarah Whaley from the Rogers Lab, for their friendship and cooperation.

I would also like to acknowledge Fulbright Colombia and the Colombian Ministry of Culture, for the scholarship that allowed me to start my graduate studies in the United States.

I will always be grateful to my family and friends, who have supported me over the years in my career goals. 


\begin{abstract}
Several important antifungal drugs inhibit the synthesis of ergosterol, a lipid that modulates the thickness, fluidity and permeability of fungal cell membranes. These include the azole antifungals, which block ergosterol biosynthesis by inhibiting lanosterol demethylase (Erg11p). The resulting depletion of cellular ergosterol and the accumulation of 'toxic' sterol intermediates are both thought to cause plasma membrane dysfunction and ultimately growth arrest. However, the effects of ergosterol depletion upon the function of intracellular membranes and organelles are not well described. The purpose of this study was to characterize the effects of azole treatment upon the integrity of the Candida albicans vacuole, and determine if, in turn, vacuolar trafficking influences azole susceptibility.

Profound fragmentation of the $C$. albicans vacuole was observed as an early consequence of azole treatment, before significant growth inhibition was observed. Using a genetic approach, we determined that vacuole fragmentation was a consequence of Erg $11 \mathrm{p}$ inhibition rather than an off-target effect of azole treatment. Moreover, the degree of vacuole fragmentation following azole treatment was influenced by Erg3p, an enzyme in the ergosterol biosynthetic pathway involved in the production of toxic sterol intermediaries upon Erg11p inhibition.
\end{abstract}

We also determined that vacuolar trafficking significantly impacts $C$. albicans susceptibility to azole antifungals and other ergosterol biosynthesis inhibitors. For instance, a vps $21 \Delta / \Delta$ mutant, blocked in membrane trafficking through the pre-vacuolar compartment (PVC), grew significantly more than wild-type controls in the presence of several azole antifungals under standard susceptibility testing conditions. Furthermore, the vps $21 \Delta / \Delta$ mutant was able to grow in the presence of the azoles despite depletion of cellular ergosterol. This phenotype resembles an exaggerated form of azole tolerance known as 'trailing growth', which has been described for some clinical isolates. In contrast, the vps $21 \Delta / \Delta$ mutant is hypersensitive to drugs that block alternate steps in ergosterol biosynthesis.

The azole tolerance phenotype of the $C$. albicans vps $21 \Delta / \Delta$ mutant was independent of known azole resistance mechanisms such as the efflux pumps Cdrlp and Mdr1p. Moreover, the azole tolerance of the $v p s 21 \Delta / \Delta$ mutant was influenced by both $\mathrm{pH}$ and incubation temperature, consistent with trailing growth phenotypes. The $C$. albicans $v p s 21 \Delta / \Delta$ mutant exhibits less plasma membrane permeabilization upon azole treatment, as determined by the release of a cytoplasmic luciferase reporter into the culture supernatant. Our results also reveal that the vps $21 \Delta / \Delta$ mutant has elevated levels of intracellular calcium and enhanced calcineurin activity, as evidenced by increased expression of a calcineurin responsive $R T A 2-G F P$ reporter construct in response to fluconazole. Furthermore, the azole tolerant phenotype of the vps $21 \Delta / \Delta$ mutant is dependent upon both calcium and calcineurin signaling. These findings underscore the importance of endosomal trafficking in determining the cellular consequences of azole 
treatment through modulation of intracellular calcium levels and calcineurin dependent responses.

While we determined that deletion of VPS21 alone was not sufficient to confer a survival advantage upon C. albicans following azole treatment in the mouse model of vaginal candidiasis, the azole susceptibility of the vps $21 \Delta / \Delta$ mutant in the mouse model disseminated infection is yet to be tested. Moreover, it is unclear how, or if the azole tolerant phenotype of the vps $21 \Delta / \Delta$ mutant relates to that of trailing clinical isolates, or if these clinical isolates have abnormal endosomal trafficking. 


\section{TABLE OF CONTENTS}

CHAPTER 1. INTRODUCTION .................................................................................

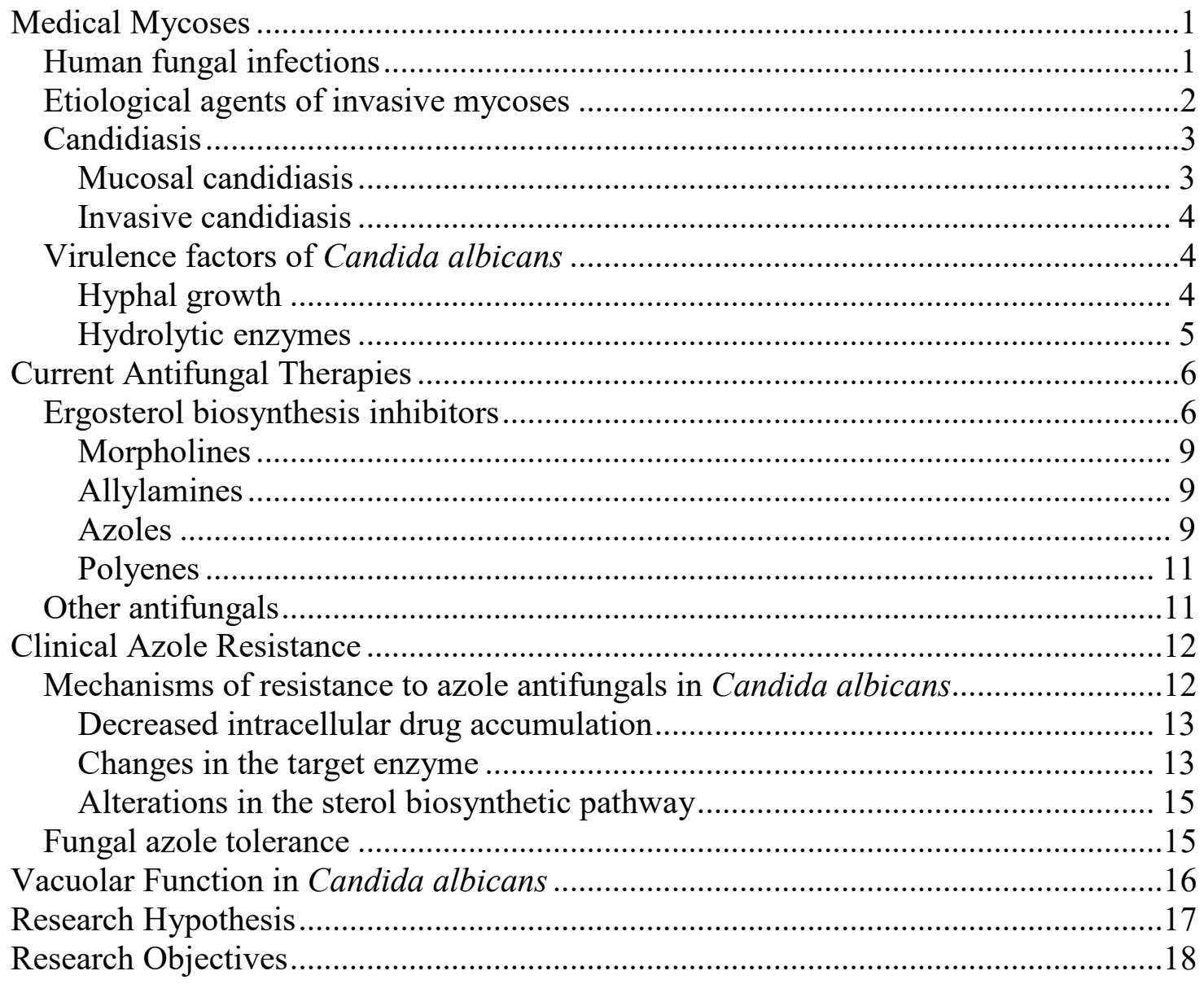

\section{CHAPTER 2. THE AZOLE ANTIFUNGALS SEVERELY DISRUPT VACUOLAR INTEGRITY IN CANDIDA ALBICANS THROUGH

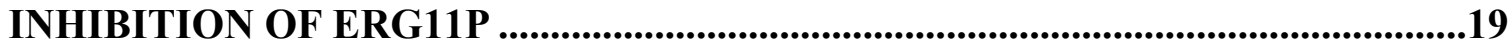

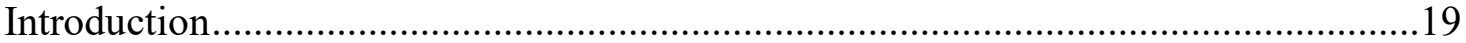

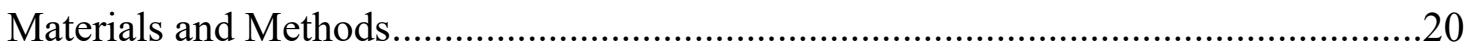

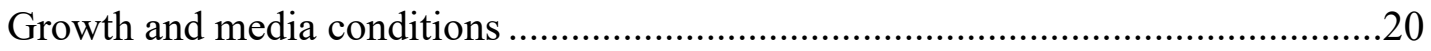

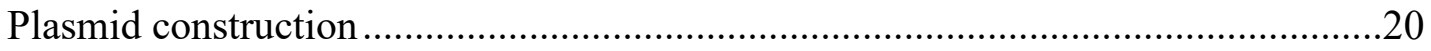

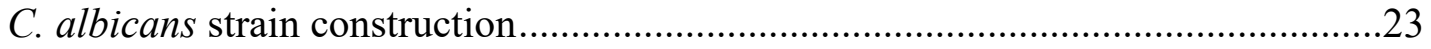

Fluorescence microscopy to determine vacuole morphology construction ...............25

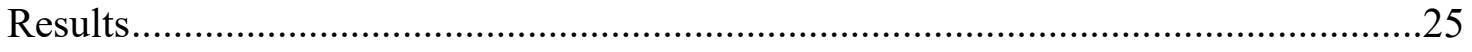

Azole antifungals cause severe vacuolar disruption in $C$. albicans ..........................25

Vacuole disruption caused by fluconazole treatment occurs before growth

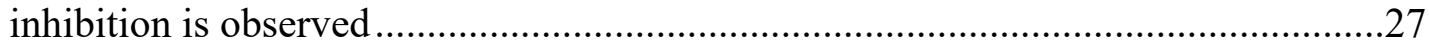

Disruption of vacuolar integrity occurs upon inhibition of Erg11p......................27

Erg $3 p$ modulates the severity of vacuole disruption in azole treated C. albicans .....33

Discussion. 


\section{CHAPTER 3. VACUOLAR TRAFFICKING THROUGH THE LATE ENDOSOME/PREVACUOLAR COMPARTMENT SIGNIFICANTLY IMPACTS AZOLE SUSCEPTIBILITY OF CANDIDA $A L B I C A N S$.}

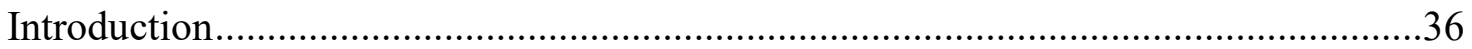

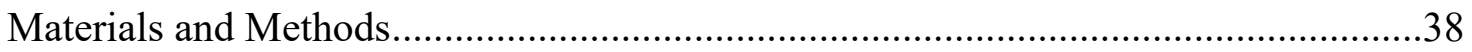

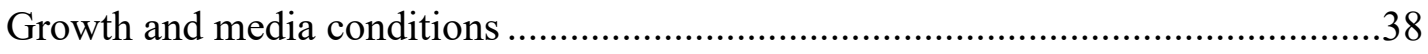

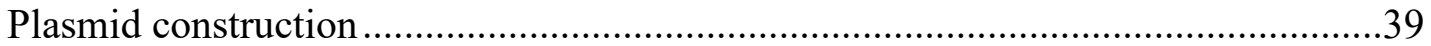

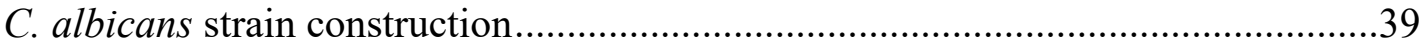

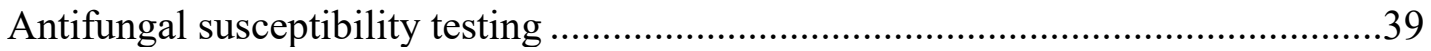

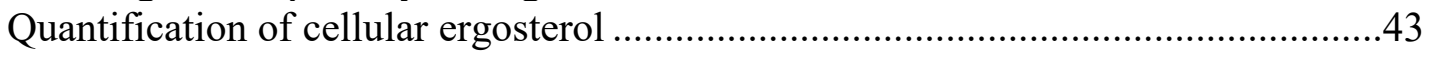

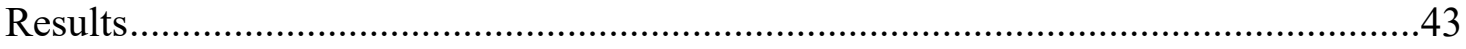

Vps21p mediated trafficking through the pre-vacuolar compartment influences fungal growth in the presence of azoles.

The VPS21 paralogs YPT52 and YPT53 do not significantly affect azole susceptibility

Susceptibility to azole antifungals is determined by the trafficking step

controlled by Vps21p rather than a Vps21p specific function

The $C$. albicans vps $21 \Delta / \Delta$ mutant exhibits an exaggerated 'trailing growth' phenotype.

The $v p s 21 \Delta / \Delta$ mutant is tolerant of Erg11p inhibition and ergosterol depletion compared to control strains.

Discussion

\section{CHAPTER 4. AZOLE TOLERANCE IN CANDIDA ALBICANS MUTANTS LACKING THE RAB GTPASE VPS21P IS DEPENDENT UPON CALCIUM SIGNALING}

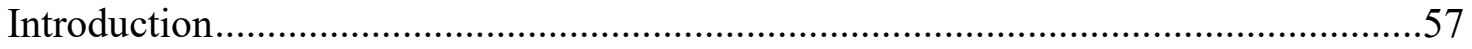

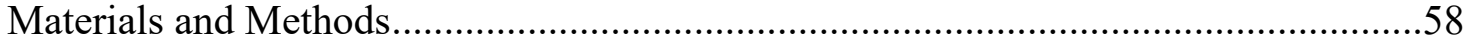

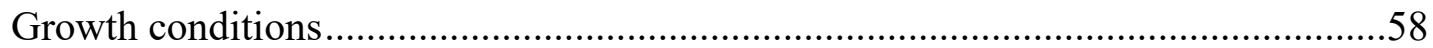

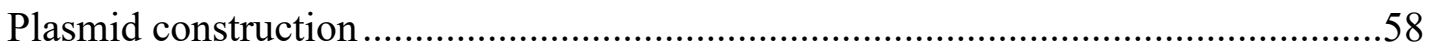

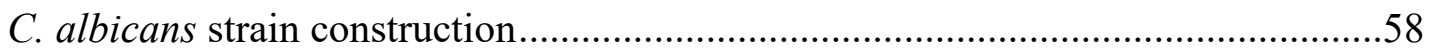

Luciferase based membrane integrity assay......................................................64

Biomass measurement and determination of cell viability .................................64

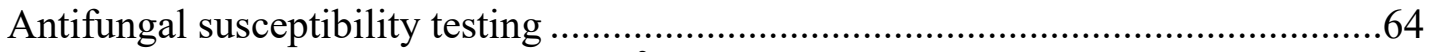

Determination of intracellular free $\mathrm{Ca}^{2+}$ levels ...................................................65

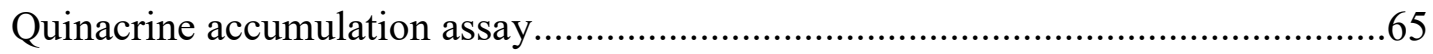

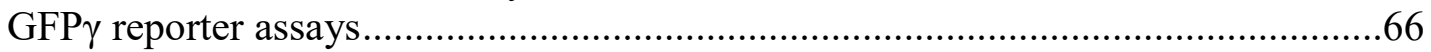

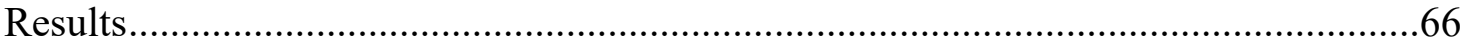

The $C$. albicans vps $21 \Delta / \Delta$ mutant is tolerant of azole induced membrane damage..66 Azole tolerance in the $C$. albicans vps $21 \Delta / \Delta$ mutant does not depend upon

Vph1p function

Azole tolerance in the C. albicans vps $21 \Delta / \Delta$ mutant is independent of the $\operatorname{Cdr} 1 \mathrm{p}$ and Mdr1p efflux pumps

The $C$. albicans vps $21 \Delta / \Delta$ mutant has elevated cytoplasmic calcium levels ............68

The $C$. albicans vps $21 \Delta / \Delta$ mutant's azole tolerance is dependent on extracellular calcium. 
The C. albicans vps $21 \Delta / \Delta$ mutant's azole tolerance is dependent upon

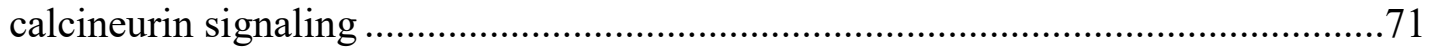

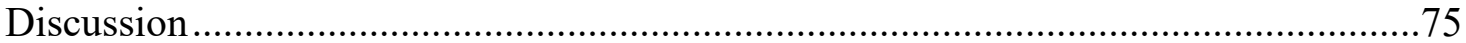

\section{CHAPTER 5. THE $C A N D I D A$ ALBICANS MUTANT LACKING THE RAB GTPASE VPS21P IS SUSCEPTIBLE TO AZOLE TREATMENT IN VIVO..........79}

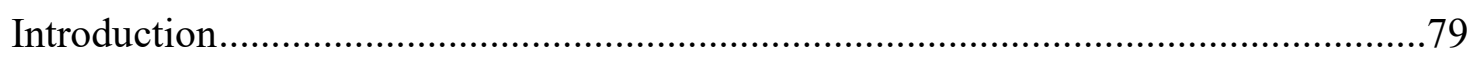

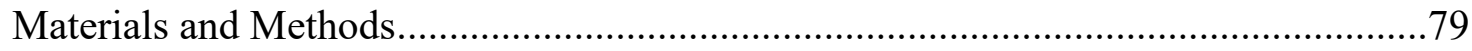

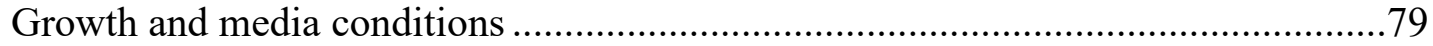

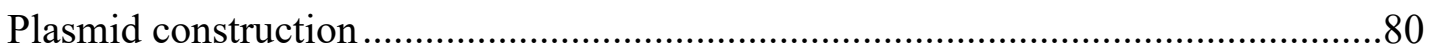

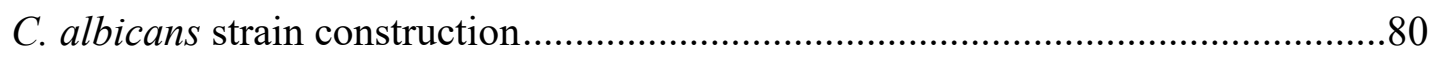

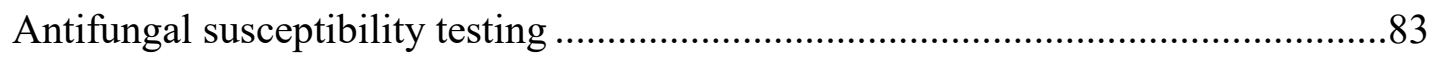

Mouse model of vaginal candidiasis and treatment of the $v p s 21 \Delta / \Delta$ mutant with

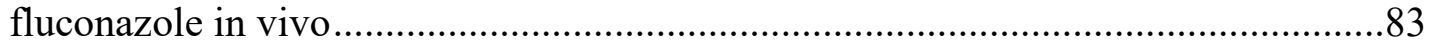

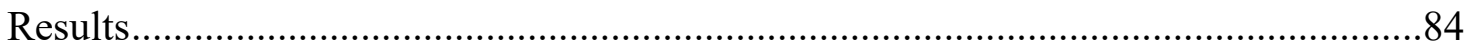

The $C$. albicans vps $21 \Delta / \Delta$ mutant is susceptible to azole treatment in a mouse model of vaginal candidiasis ...................................................................... 84

Overexpression of ERG11 antagonizes azole tolerance in the C. albicans

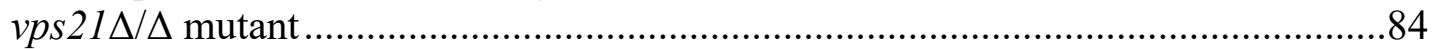

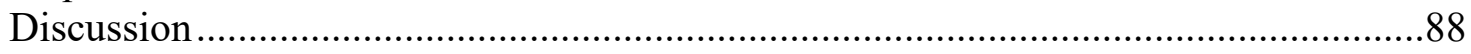

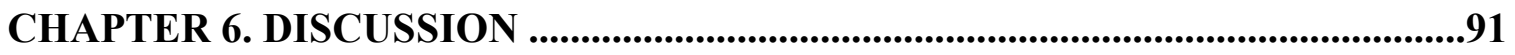

Azole Antifungals and Vacuolar Integrity.......................................................91

Vacuolar Trafficking as a Determinant of the Antifungal Efficacy of Ergosterol

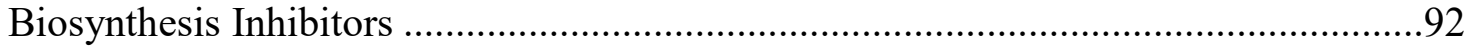

Connecting the Dots: Vacuolar Trafficking, $\mathrm{Ca}^{2+}$ Homeostasis and Azole Tolerance..93

Concluding Remarks................................................................................ 98

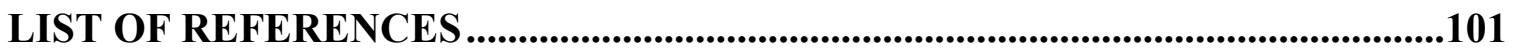

APPENDIX A. SUPPLEMENTAL FIGURES FOR CHAPTER 3 ........................117

APPENDIX B. SUPPLEMENTAL FIGURES FOR CHAPTER 4...........................121

APPENDIX C. LIST OF STRAINS DESCRIBED IN CHAPTER 6.......................123

VITA 


\section{LIST OF TABLES}

Table 2-1. List of oligonucleotides used in the study described in Chapter 2. ............21

Table 2-2. $\quad$ List of strains used in the study described in Chapter 2. .............................24

Table 3-1. List of oligonucleotides used in the study described in Chapter 3. .............40

Table 3-2. List of strains used in the study described in Chapter 3. ...........................41

Table 4-1. List of oligonucleotides used in the study described in Chapter 4. .............59

Table 4-2. List of strains used in the study described in Chapter 4. ..........................61

Table 5-1. List of oligonucleotides used in the study described in Chapter 5.............81

Table 5-2. List of strains used in the study described in Chapter 5. ...........................82 


\section{LIST OF FIGURES}

Figure 1-1. Ergosterol biosynthesis pathway.............................................................

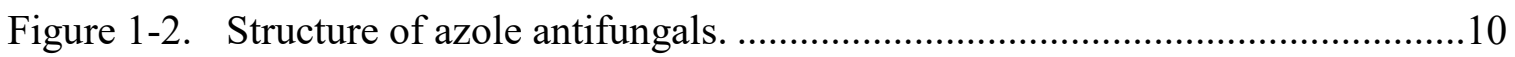

Figure 1-3. Azole resistance mechanisms in fungi.....................................................14

Figure 2-1. Azole antifungals cause severe disruption of the C. albicans vacuole.........26

Figure 2-2. The integrity of the C. albicans vacuole is severely compromised after

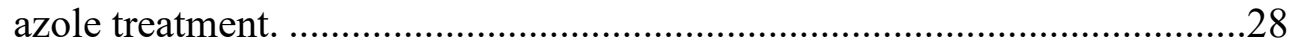

Figure 2-3. Inhibition of Erg11p causes vacuolar fragmentation in C. albicans.............29

Figure 2-4. Azole treatment severely disrupts the integrity of C. albicans vacuole. .......31

Figure 2-5. Loss of Erg3p reduces the impact of azole treatment upon vacuolar integrity in C. albicans.

Figure 3-1. Membrane trafficking pathways to the vacuole of C. albicans analyzed in this study.

Figure 3-2. Endosomal trafficking influences the growth of C. albicans in the presence of miconazole.

Figure 3-3. Endosomal trafficking defects alter C. albicans growth in the presence of fluconazole.

Figure 3-4. The Rab GTPase Vps21p impacts C. albicans growth in the presence of the triazole itraconazole and the imidazole ketoconazole

Figure 3-5. Endosomal trafficking impacts $C$. albicans susceptibility to other ergosterol biosynthesis inhibitors.

Figure 3-6. The Vps21p paralogs Ypt52p and Ypt53p have minimal effect upon $C$. albicans growth in the presence of fluconazole.

Figure 3-7. The C. albicans pep $12 \Delta / \Delta$ mutant has reduced susceptibility to fluconazole.

Figure 3-8. The dose-response growth profile of the $v p s 21 \Delta / \Delta$ mutant to fluconazole is distinct from that of an azole resistant clinical isolate.

Figure 3-9. The C. albicans vps $21 \Delta / \Delta$ mutant shows features consistent with an exaggerated trailing growth-like phenotype. 
Figure 4-1. The C. albicans vps $21 \Delta / \Delta$ mutant has delayed membrane damage following azole treatment.

Figure 4-2. Deletion of gene encoding for the V-ATPase subunit Vph1p from the vps $21 \Delta / \Delta$ mutant does not restore sensitivity to azole antifungals.

Figure 4-3. Growth of the C. albicans vps $21 \Delta / \Delta$ mutant in the presence of fluconazole does not depend upon the Cdrlp efflux pump.

Figure 4-4. The C. albicans vps $21 \Delta / \Delta$ mutant has elevated intracellular levels of $\mathrm{Ca}^{2+}$.

Figure 4-5. The $C$. albicans vps $21 \Delta / \Delta$ mutant's azole tolerance depends upon extracellular calcium.

Figure 4-6. Inhibition of calcineurin signaling suppresses the vps $21 \Delta / \Delta$ mutant's azole tolerance.

Figure 5-1. The C. albicans vps $21 \Delta / \Delta$ mutant is susceptible to azole treatment in a mouse model of vaginal candidiasis.

Figure 5-2. Overexpression of efflux pumps does not synergize or antagonize azole tolerance in the $C$. albicans vps $21 \Delta / \Delta$ mutant.

Figure 5-3. Overexpression of ERG11 reduces azole tolerance in the C. albicans vps $21 \Delta / \Delta$ mutant.

Figure 6-1. The vacuolar calcium pump Pmc1p and the $\mathrm{Ca}^{2+} / \mathrm{H}^{+}$exchanger Vcx $1 \mathrm{p}$ do not impact $C$. albicans susceptibility to fluconazole.

Figure 6-2. Accepted model of $\mathrm{Ca}^{2+}$ dependent response to azole antifungals in $C$. albicans.

Figure 6-3. Proposed model for the $\mathrm{Ca}^{2+}$ dependent azole tolerance in the $C$. albicans vps $21 \Delta / \Delta$ mutant.

Figure 6-4. Disruption of the ALP trafficking pathway in the C. albicans vps $21 \Delta / \Delta$ mutant eliminates azole tolerance.

Figure A-1. The C. albicans Vps21p paralogs Ypt52p and Ypt53p do not significantly affect susceptibility to miconazole.

Figure A-2. Deletion of VPS28 does not affect C. albicans susceptibility to fluconazole.

Figure A-3. The composition of sterols extracted from fluconazole-treated $v p s 21 \Delta / \Delta$ mutant is similar to that of the isogenic control. 
Figure B-1. Fluconazole induced expression of GFP from the RTA2 promoter is

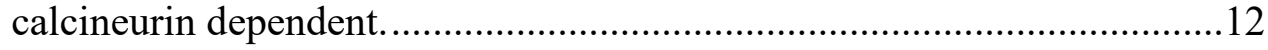

Figure B-2. The calcineurin signaling inhibitor FK506 eliminates the C. albicans vps $21 \Delta / \Delta$ mutant's azole tolerance. .................................................122 


\section{LIST OF ABBREVIATIONS}

AAALAC

$\mathrm{ABC}$

AIDS

ALP

AMD

ATP

$\mathrm{CDCl}_{3}$

CFU

CLSI

CPP

CPY

CsA

DMSO

DNA

Dox

EGTA

ESCRT

FITC

FKBP

FLU

gDNA
Association for Assessment and Accreditation of Laboratory Animal Care

ATP-binding cassette

Acquired immune deficiency syndrome

Alkaline phosphatase

Amiodarone

Adenosine triphosphate

Deuterated chloroform

Colony forming units

Clinical and Laboratory Standards Institute

Cpyl prepropeptide

Carboxypeptidase Y

Cyclosporine A

Dimethyl sulfoxide

Deoxyribonucleic acid

Doxycycline

Ethylene glycol tetraacetic acid

Endosomal sorting complexes required for transport

Fluorescein isothiocyanate

FK506 binding protein

Fluconazole

Genomic DNA 


\begin{tabular}{|c|c|}
\hline GFP & Green fluorescent protein \\
\hline HACS & High-affinity $\mathrm{Ca}^{2+}$ influx system \\
\hline HEPES & 4-(2-Hydroxyethyl)piperazine-1-ethanesulfonic acid \\
\hline HIV & Human immunodeficiency virus \\
\hline ICU & Intensive care unit \\
\hline IL-1 $\beta$ & Interleukin 1 beta \\
\hline $\mathrm{MCZ}$ & Miconazole \\
\hline MFS & Major facilitator superfamily \\
\hline MIC & Minimum inhibitory concentration \\
\hline MOPS & 3-(N-morpholino) propanesulfonic acid \\
\hline MVB & Multivesicular bodies \\
\hline NIS & Nisoldipine \\
\hline Nluc & Nano luciferase ${ }^{\circledR}$ \\
\hline NMR & Nuclear magnetic resonance \\
\hline NSF & N-ethylmaleimide-sensitive factor \\
\hline $\mathrm{OD}_{600 \mathrm{~nm}}$ & Optical density at $600 \mathrm{~nm}$ \\
\hline ORF & Open reading frame \\
\hline p.i. & Post-infection \\
\hline PBS & Phosphate buffered saline \\
\hline $\mathrm{PC}$ & Phase contrast \\
\hline PCR & Polymerase chain reaction \\
\hline $\mathrm{PVC}$ & Pre-vacuolar compartment \\
\hline ROS & Reactive oxygen species \\
\hline
\end{tabular}




$\begin{array}{ll}\text { RPMI } & \text { Roswell Park Memorial Institute } \\ \text { SAP } & \text { Secreted aspartyl proteinase } \\ \text { SDS } & \text { Sodium dodecyl sulfate } \\ \text { SNARE } & \begin{array}{l}\text { Soluble N-ethylmaleimide-sensitive factor activating protein } \\ \text { receptor }\end{array} \\ \text { TMS } & \text { Tetramethylsilane } \\ \text { TRITC } & \text { Tetramethylrhodamine isothiocyanate } \\ \text { V-ATPase } & \text { Vacuolar H } \text { H }^{+} \text {ATPase } \\ \text { VVC } & \text { Vulvovaginal candidiasis } \\ \text { WT } & \text { Wild-type } \\ \text { YNB } & \text { Yeast nitrogen base } \\ \text { YPD } & \text { Yeast extract-peptone-dextrose } \\ \text { 5-FOA } & \text { 5-Fluoroorotic acid }\end{array}$




\title{
CHAPTER 1. INTRODUCTION
}

\author{
Medical Mycoses
}

\section{Human fungal infections}

An estimated of 1.5 million fungal species exist in nature (1), with only 70,000 species described so far (2) and around 300 that have been reported to cause disease in humans (3). Within the last group, only a few are common pathogens of humans and other mammals (2). The latter situation seems to indicate a high degree of resistance of mammals to fungi which may be due to both an effective immune system against these organisms and an elevated basal body temperature that can be increased upon infection, thus limiting the opportunities for development of fungal infections (2). However, over the last few decades, fungi have emerged as important public health concerns (4) due to the increasing numbers in immunocompromised populations (including those suffering from cancer, HIV infection, diabetes, immunosuppressive therapy and cystic fibrosis) (5), invasive medical procedures (6) and global environmental changes (2), among other factors. In addition, fungi are eukaryotic organisms sisters to animals as part of Opisthokonta and hence they share a great number of features with mammalian cells, narrowing the number of therapeutic targets that could be exploited to fight fungal infections. In this regard, the number of antifungal drugs available remains limited.

The impact of fungal infections on human health is widely underestimated. Most human fungal infections are regarded as opportunistic and are associated with predisposing factors that include host immunosuppression, organ transplant, aging, AIDS and disruption of the microbiota (7). Human fungal pathogens cause a variety of diseases that include cutaneous lesions and acute pulmonary infections in immunocompetent individuals and inflammatory and life-threatening infections in immunocompromised patients (8). Superficial infections are the most common forms of disease caused by fungi and affect $\sim 1.7$ billion people worldwide (6). They are characterized as benign and selflimiting, do not involve tissue invasion and occur on keratinized tissue such as nails, hair and outer layers of the skin (9). However, infections of nails and skin can be distressing and unappealing, and in many cases the patients can experience pain (10). Common examples of superficial fungal infections include athlete's foot which affects 1 in 5 adults, and onychomycosis which affects from 5 to $25 \%$ of healthy individuals and increases its incidence in the elderly $(5,6,11)$. This category of fungal infections are mostly caused by dermatophytes (mainly Trichophyton spp, Microsporum spp and Epidermophyton spp) and to a lesser extent by yeast of the genera Candida spp and Malassezia spp, and in fewer cases by molds (9).

Subcutaneous fungal infections affect the dermis and subcutaneous tissue and usually result from invasion by any of several species of fungi after local trauma (12). Sporotrichosis (caused by Sporothrix schenkii), mycetoma (caused by Madurella mycetomatis, M. grisea, Pseudallescheria boydii among others), and 
chromoblastomycosis (caused by Fonsecaea pedrosi, F. compacta, Phialophora verrucosa, among others) are the most common subcutaneous mycoses. These mycoses occur more frequently in tropical and subtropical regions of the planet, where the etiological agents are widely distributed in soil, plants and animals (12). As for other fungal infections, immunocompromised individuals are at increased risk of developing subcutaneous mycoses (12).

Life-threatening infections are characterized by fungal dissemination to deep tissue and internal organs, causing symptoms such as fever and septic shock (5). This class of illness occurs in people having well established risk factors that include an impaired immunity due to genetic predisposition, neutropenia, immunosuppressive therapy and $\operatorname{AIDS}(5,6)$. Although invasive fungal infections occur with lower frequency than superficial infections, in immunocompromised individuals they are associated with high mortality rates that often exceed $50 \%$, leading to an estimated 1.5 million deaths worldwide per year, despite the availability of several antifungal drugs (6). The death toll is especially high in settings such as Sub-Saharan Africa where the incidence of HIV/AIDS is high and the diagnosis and treatment for invasive fungal infections is limited. In addition, the cost of treating systemic fungal infections is high; in the United States alone, the annual hospital cost due to invasive fungal infections is estimated to be $\$ 1.89$ billion (13).

\section{Etiological agents of invasive mycoses}

Invasive fungal infections in humans can be caused by a wide range of fungi, including members of the genera Histoplasma, Coccidioides, Blastomyces, Paracoccidioides, Fusarium spp, Sporothrix, Penicillium, Scedosporium, Mucor, Rhizopus and Rhizomucor (14-17). However, over $90 \%$ of all cases of invasive fungal infections are caused by species of Aspergillus, Cryptococcus, Candida and Pneumocystis $(6,18,19)$. Many of these fungi are widely distributed in nature or are considered part of the normal microbiota (e.g., several Candida species), and thus controlling the exposure of susceptible patients is impractical.

Due to the worldwide distribution of Aspergillus, most people breathe its conidia (spores) on a daily basis without harmful consequences to the immunocompetent populations. However, inhalation of Aspergillus spores can cause invasive infections in immunocompromised individuals and those with chronic obstructive pulmonary disease (6). Aspergillosis is characterized by the germination of conidia, followed by growth of invasive hyphae in the lungs (20). The annual number of cases of aspergillosis is estimated to be over 200,000 worldwide, with mortality rates of $50 \%$ in patients with correct diagnosis and treatment, but it can reach almost $100 \%$ if the diagnosis is not performed in a timely manner (6). In addition, fungal allergy and A. fumigatus infection have been linked to the development of severe asthma $(6,21,22)$. Accordingly, the global burden of severe asthma due to fungal sensitization is estimated to affect up to 12 million adults and contribute to the mortality/morbidity due to asthma, which reaches over 100,000 cases per year $(6,23)$. 
Cryptococcus is a globally distributed fungal pathogen. After inhalation, Cryptococcus spores or desiccated yeast cells enter the respiratory tract where they can be eradicated or isolated inside granulomas by the host's immune response, or cause an asymptomatic infection within the lungs $(24,25)$. However, Cryptococcus cells can disseminate hematogenously to other organs including the eyes, urinary tract, bones and central nervous system, if host immunity becomes dysfunctional (25). Infection is common in patients whose risk factors include AIDS and immunosuppressive therapy (6), with mortality rates of 15 to $20 \%$ in developed countries and 55 to $70 \%$ in Latin America and sub-Saharan Africa. For cryptococcal meningitis alone, the annual global burden is estimated to be over one million cases, with over 620,000 deaths in subSaharan Africa alone $(6,26)$ where the access to antiretroviral therapy against HIV/AIDS is severely limited.

A proper and timely diagnosis is imperative to treat invasive fungal infections (27). Despite this importance, difficulties in diagnosing these infections remain and consequently the high mortality rates of invasive fungal infections (28). The diagnostic tools available for fungal infections lack sensitivity and/or have limited specificity (29) and access is limited in low income countries (6). Histopathology continues to be a rapid and cost-effective means of providing a presumptive or definitive diagnosis of an invasive fungal infection; however, it allows only the identification of yeast or molds and often lacks enough sensitivity to determine the infecting specie or strain $(27,28)$. Blood and deep-tissue culture are insensitive and slow; for example, blood cultures from patients with deep-seated invasive infections often give negative results and blood cultures in the diagnosis of invasive candidiasis or aspergillosis have sensitivities of $\sim 50 \%$ and $0 \%$, respectively $(6,18)$.

\section{Candidiasis}

Candida species are normal constituents of the microbiota on the skin, oral cavity, gastrointestinal tract and vagina of humans (30). However, in susceptible individuals they are the etiological agents of a group of diseases known as candidiasis, that vary from superficial mucosal conditions such as oral thrush and vaginitis, to life-threatening systemic infections (31). Out of two hundred known Candida species, over a dozen species can cause disease in humans, and are collectively considered as the second most abundant causes of fungal disease worldwide (6). In terms of incidence in all disease manifestations, C. albicans is the leading species among its genera (6).

\section{Mucosal candidiasis}

Mucosal candidiasis is characterized by profusive growth of Candida on mucosal surfaces such as the vagina, esophagus, oropharynx and urinary tracts (5). Excessive Candida growth in the oral cavity is known as "oral thrush" and can include infection on the mucosa of the mouth and throat. This class of infections is common in immunocompromised patients (5); approximately $70 \%$ of HIV/AIDS patients develop 
oropharyngeal candidiasis (32), contributing to a global burden estimated to be around 10 million annual cases of oral thrush worldwide (6). Vulvovaginal thrush is common in otherwise healthy women; it is estimated that 50 to $75 \%$ of all women suffer from at least one episode of vulvovaginal candidiasis (VVC) in their childbearing years, from which 40 to $50 \%$ will experience a subsequent infection (33). Notably, 5 to $8 \%$ of women suffer of recurrent VVC, with 3 or more episodes per year (34). Mucosal infections of the oral cavity can be unpleasant and uncomfortable when ingesting meals (35), while infections of the vaginal tract can cause general discomfort, itching and burning sensation (36). $C$. albicans, C. glabrata, Aspergillus spp., Cryptococcus spp., and Blastomyces spp. among other fungi, are able to cause mucosal infections (37).

\section{Invasive candidiasis}

Invasive candidiasis occurs when any of several Candida species reach the blood and organs such as the heart, brain, eyes, and liver, generally due to breach of the integument or impaired immune function (38). Several predisposing factors can increase the risk of invasive Candida infections and include a severely immunocompromised state, the use of catheters, prolonged stays in intensive care units, severe trauma or invasive clinical procedures such as major gut surgery or liver transplantation, among others (6). Candida species are the most common invasive fungal pathogens with $C$. albicans accounting for more than $50 \%$ of all Candida infections (39) and a mortality rate of $\sim 46-75 \%(6,39)$.

Candida bloodstream infection, or candidemia, is the most common form of invasive candidiasis with an estimated 400,000 bloodstream infections occurring worldwide per year (6). In the United States, Candida spp. are ranked the fourth most common cause of nosocomial bloodstream infections in the intensive care unit (ICU) (40). Approximately 10,500 to 42,000 total cases of candidemia are estimated to occur annually in the United States (4). Moreover, between 2,800 and 11,200 deaths each year may be associated with nosocomial candidemia based on a crude mortality rate of $40 \%$ for Candida bloodstream infections (4). The annual economic burden for this type of infection is estimated to exceed one billion dollars (41).

\section{Virulence factors of Candida albicans}

During both mucosal and disseminated infection, C. albicans uses an array of virulence attributes that include the ability to switch from yeast to hyphae, and secretion of hydrolytic enzymes, among others, that promote host tissue damage (42).

\section{Hyphal growth}

C. albicans is a polymorphic fungus with the ability to switch reversibly between the ovoid-shaped budding yeast, pseudohyphae, and hyphae (43), a process often referred as morphogenesis. In the human host, $C$. albicans can be found as yeast, pseudohyphae or in its invasive filamentous hyphal form $(30,43,44)$. The morphological plasticity of $C$. 
albicans is considered a virulence determinant (45), since the ability to switch between both yeast and hyphae have key roles in pathogenesis. In this regard, it is assumed that the yeast form is better suited for dissemination to bloodstream and tissues due to its oval shape and relatively small size (46), while the hyphal form is better adapted for tissue invasion and damage as well as escape from immune cells as hyphae are capable of rupturing host cell membranes $(45,47,48)$. C. albicans mutants that are unable to form hyphae are not only typically avirulent in mouse models of disseminated or mucosal candidiasis. They also have decreased ability to invade and damage organs such liver and pancreas, have reduced ability to penetrate mucosal surfaces and are also prone to be killed by macrophages in vitro (49-52). In addition, strains that are unable to grow in the yeast form also have reduced virulence $(53,54)$, further supporting the importance of the ability of $C$. albicans to transition between yeast and hyphal forms. Importantly, since yeast and hyphal cells can both be found in infected organs, with a predominance of one particular form depending on the target organ (55), it has been suggested that both have a role in disease progression (32).

\section{Hydrolytic enzymes}

Several enzymes that are secreted by $C$. albicans have been associated with virulence. Among these, secreted aspartyl proteases (SAPs) and phospholipases are the best characterized.

Secreted aspartyl proteases. Aspartyl proteases are proteinases that have two highly conserved aspartate residues in the active site that are used to hydrolyze peptide bonds in proteins (56). The family of secreted aspartyl proteinases (SAP) in C. albicans contains at least ten members between 35 and $50 \mathrm{kDa}$ in size (56-58), whose primary function is to degrade proteins for nutrient acquisition (56). SAPs are synthesized as preproenzymes that are processed in the Golgi and released into the extracellular space via the secretory pathway. They have been linked not only to invasion and damage of host tissues, but also to adherence (59); SAPs are able to degrade host membrane proteins in order to facilitate invasion (56). This function also seems to confer a protective role against the host's immune system by degrading membrane proteins and proteins secreted by the host as part of the immune response against the fungus (56), including lactoferrin, immunoglobulins, complement components and cathepsin D $(56,59-61)$. SAP activity can induce an acute inflammatory immune response in the vaginal environment (62) and has been shown to lead to activation of the proinflammatory cytokine IL-1 $\beta$ (63).

Although there are multiple SAPs, the requirement of each one for disease development seems to vary between different infection sites. For example, Sap1p-Sap6p appear to be required for virulence in models of disseminated candidiasis $(64,65)$, but the requirement of each one of them seems to vary in several in vitro models of mucosal disease $(66,67)$. Sap2 has been shown to be required for disease progression in a rat model of vaginal candidiasis (68), and to induce inflammatory responses by itself in a mouse model of vaginal candidiasis (62). In addition, several studies have shown a positive correlation between the level of SAP activity and virulence of mucosal isolates of $C$. albicans $(56,69-71)$. Clinical isolates of $C$. albicans from symptomatic patients 
with vaginal candidiasis were shown to have higher SAP activity in vitro than isolates from asymptomatic patients $(69,70,72)$, as did clinical isolates from HIV-positive patients with oral candidiasis versus isolates from HIV-negative patients.

Phospholipases. Phospholipases are enzymes that hydrolyze acyl and phosphate esters in phospholipids. In C. albicans phospholipases have been associated with penetration and damage of host tissue by degradation of the host's cell membrane phospholipids (73). Several types of phospholipases, including phospholipase $A_{1}, A_{2}, C$, and $\mathrm{D}$, lysophospholipase, and lysophospholipase-transacylase, have been identified in $C$. albicans $(74,75)$. However, phospholipase B1 (Plb1p) is the only phospholipase that has been shown to be required for virulence in vivo. Deletion of $P L B 1$ led to attenuation of virulence in a mouse model of disseminated (73) and intragastric candidiasis (76). Moreover, Plb1p has been detected at the hyphal tips during penetration of tissues (77).

\section{Current Antifungal Therapies}

A large number of fungal pathogens can cause disease in humans, each of them with different susceptibilities to the antifungal drugs available. Of concern is that current antifungal therapies have led to only modest success in reducing the high mortality rates caused by invasive fungal infections, in part due to drug toxicity, narrow spectrum of activity and drug resistance (6). In addition, the difficulties in providing a timely and accurate diagnosis of invasive fungal infections further limits the chances of reducing those mortality rates by delaying the selection of appropriate treatments (78).

\section{Ergosterol biosynthesis inhibitors}

A number of enzymes in the ergosterol biosynthetic pathway (Figure 1-1A) are targeted by several classes of antifungal drug currently in clinical use; these include the morpholines, allylamines and azoles. The membrane lipid ergosterol (Figure 1-1B), the primary fungal sterol, is important to modulate the thickness, fluidity and permeability of cellular membranes $(79,80)$. Ergosterol is crucial for cellular processes such as sporulation, pheromone signaling and plasma membrane fusion and also contributes to the correct function of a wide variety of membrane-bound proteins (81). The function of a multitude of proteins including those responsible for nutrient uptake requires ergosterol (82). In addition, ergosterol is a major component of secretory vesicles in Saccharomyces cerevisiae. Loss of ergosterol in the plasma membrane leads to defects that include loss of fluidity and function that in turn inhibits cell growth and cell division (83). Genetic or pharmacological inhibition of ergosterol biosynthesis can also impact mitochondrial morphogenesis and function in yeast. Several ergosterol biosynthetic (erg) mutants have aberrant mitochondrial morphologies and a number of ergosterol biosynthesis inhibitors are known to induce reactive oxygen species (ROS) and reduce mitochondrial ATPase activity (84-86). Moreover, deletion of the genes responsible for early steps in ergosterol biosynthesis in fungi is lethal, underscoring the importance of the synthesis of this lipid (87). 
Figure 1-1. Ergosterol biosynthesis pathway.

(A) A linear model of the ergosterol biosynthetic pathway is depicted. The enzymes that catalyze each step and the intermediates produced (boldface) are shown. The classes of antifungals that block the ergosterol biosynthetic pathway and their targets are displayed in red. (B) The structures of ergosterol (left) and its functional analog in animal cells cholesterol (right) differ from each other by two double-bonds and a methyl group at C24. 
A.

Acetyl CoA (2x)

Erg10p

Acetoacetyl-CoA

Erg13p

HMG-CoA

Hmglp

Mevalonic Acid

Erg12p

Mevalonate-5-phosphate

Erg8p

Mevalonate-5-pyrophosphate

Erg19p $\downarrow$

Isopentenyl-pyrophosphate

Erg20p

Farnesyl-pyrophosphate (2x)

Erg9p $\downarrow$

Squalene

Erglp $\downarrow \longmapsto$ Allylamines

2,3-Oxidosqualene

$\operatorname{Erg} 7 \mathrm{p}$

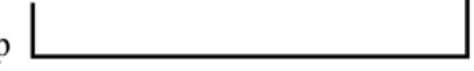

B.

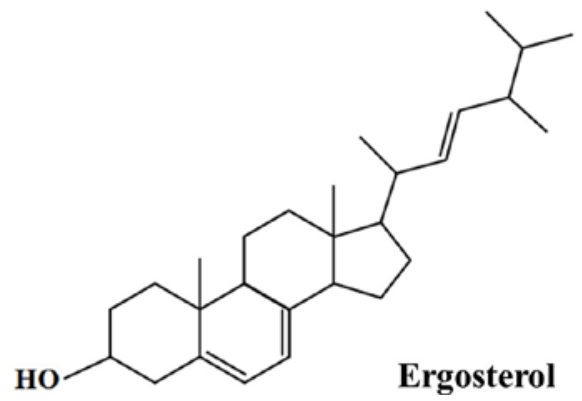

Lanosterol

Erg11p $\downarrow \longmapsto$ Azoles

4,4-Dimethylcholesta-8,14,24-trienol

Erg24p

4,4-Dimethyl zymosterol

Erg25p
Erg26p
Erg27p

4,4-Methyl zymosterol

$\operatorname{Erg} 25 \mathrm{p}$

Erg26p

Erg27p

Zymosterol

Erg6p $\downarrow$

Fecosterol

Erg2 $\mathrm{p} \downarrow$

Episterol

Erg3p

Ergosta-5,7,24(28)-trienol

Erg5p

Ergosta-5,7,22,24(28)-tetraenol

Erg4p

Ergosterol $\longmapsto$ Polyenes

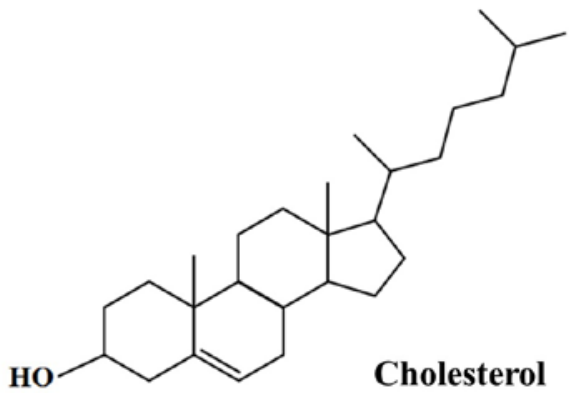




\section{Morpholines}

The morpholine antifungals, which include the topical amorolfine and the agricultural pesticides tridemorph and fenpropimorph, inhibit two enzymes within the late ergosterol biosynthetic pathway, sterol- $\Delta^{7}-\Delta^{8}$-isomerase and sterol- $\Delta^{14}$-reductase, the products of the ERG2 and ERG24 genes, respectively (Figure 1-1A) (88). Growth inhibition by these drugs is believed to be the result of the accumulation of sterol precursors in the fungal cell and/or the depletion of the major fungal sterol ergosterol (88). In vitro susceptibility testing indicates that amorolfine has higher efficacy against dermatophytes and dimorphic fungi. However, when tested in animal models of fungal infections, amorolfine only showed activity in animal models of dermatophytosis and vaginal candidiasis (89).

\section{Allylamines}

Allylamines inhibit the product of the gene ERG1, squalene epoxidase, which catalyzes the epoxidation of squalene to 2,3-oxidosqualene (Figure 1-1A) (90). The resulting squalene accumulation and ergosterol depletion affect the integrity of the plasma membrane and increases cell permeability, which ultimately leads to growth inhibition and cell death (90). This group of drugs includes terbinafine and naftifine. Terbinafine, is routinely used in the treatment of nail infections (83).

\section{Azoles}

The azoles are the most widely used and important class of antifungal drugs, which inhibit lanosterol-14-demethylase (Erg11p, Figure 1-1A), a cytochrome P450 enzyme (81). A nitrogen atom in the azole ring binds to the iron atom in the heme group of the active site (91). Inhibition of Erg $11 \mathrm{p}$ leads to the accumulation of $14 \alpha$-methylated sterol intermediates that are converted by Erg3p to 14 $\alpha$-methylergosta-8,24(28)-dien$3 \beta, 6 \alpha$-diol, which accumulates in the plasma membrane resulting in increased permeability, defective membrane fluidity and ultimately growth inhibition (92).

The azoles can be divided into two groups based on the number of nitrogen atoms in the azole ring. Imidazoles contain two nitrogen atoms while triazoles contain three (Figure 1-2A and B) $(5,93)$. Imidazoles were the first subclass to be developed for antifungal therapy. Several imidazoles such as miconazole (Figure 1-2B, left) and clotrimazole have been shown to possess additional mechanisms in addition to Erg11p inhibition that mediate their antifungal activity, including direct membrane damage at high concentration, inducing ROS production, actin cytoskeleton damage and impairment of ATP production. These additional mechanisms may explain why several imidazoles are cidal at high concentrations. Although effective antifungals, the elevated toxicity of imidazoles when administered orally or parentally restrict their use to topical applications. In addition, several of them have poor intestinal absorption, cannot cross the cerebrospinal barrier and cause severe side effects including liver and gastrointestinal complications. These shortcomings led to the development of the triazoles. The first triazole available for clinical use was fluconazole (Figure 1-2B, right) in 1990. It is 
A. Imidazole Ring

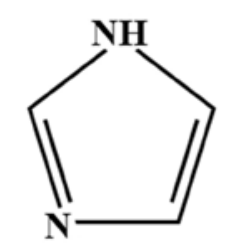

B.<smiles>CCCCOC(Cn1ccnc1)c1ccc(Cl)cc1Cl</smiles>

Triazole Ring

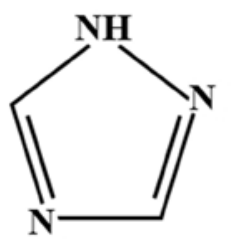

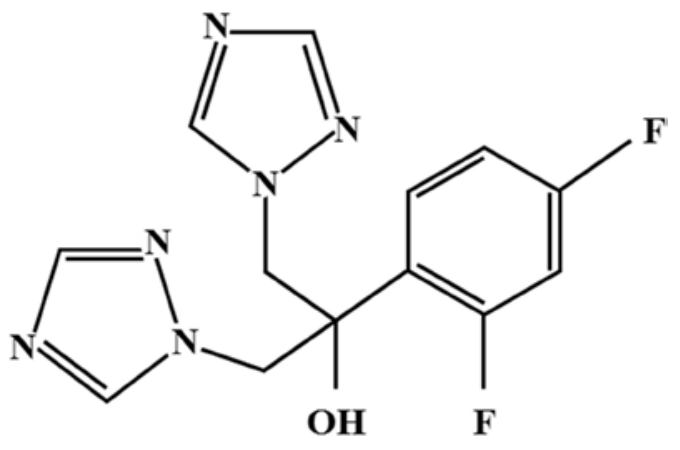

Fluconazole

Figure 1-2. Structure of azole antifungals.

(A) Azole antifungals are classified with regard to the number of nitrogen atoms in the azole rings: imidazoles (left) contain two nitrogen atoms while triazoles (right) contain three nitrogen atoms in the azole ring. (B) The chemical structure of the imidazole drug miconazole (left) and the triazole antifungal fluconazole (right). 
highly soluble in water favoring its oral and parenteral use, has good intestinal absorption, can cross the blood-brain barrier and has a broad spectrum of activity against several groups of fungi. Although the triazoles have proven efficacy with good safety profiles, azole resistant fungi have emerged from their continuous use (94).

The third generation of azole antifungals, also called the "second-generation" triazoles, was developed in order to overcome the limitations of the "first-generation" triazoles, which included limited spectrum of activity, acquired resistance and toxicity among others. Examples of third-generation triazoles include voriconazole, ravuconazole and posaconazole, which possess increased potency, wider spectrum of activities, better safety profiles, and they represent new alternatives against invasive fungal infections such as aspergillosis in immunocompromised populations $(93,95)$.

\section{Polyenes}

Polyenes antifungals include amphotericin B (AmB), natamycin and nystatin. Polyenes bind directly to plasma membrane ergosterol (Figure 1-1A and B), with a secondary mechanism that consists of the formation of channels in the plasma membrane that enhances cell killing (96-99) due to leakage of ions and loss of the proton gradient $(100,101)$. In addition, polyenes are able to cause inhibition of plasma membrane chitin synthase at high concentration (102). Polyenes are effective against a multitude of fungal pathogens that include Candida, Aspergillus, Zygomycetes, Cryptococcus and other dimorphic species (102). However, polyenes are also able to bind the ergosterol analog cholesterol (Figure 1-2B) on human cell membranes, which explains to a certain extent its toxicity (103). For instance, AmB is highly toxic when administered systemically, with renal impairment as the main side effect $(102,104)$.

\section{Other antifungals}

Another class of antifungals of clinical importance consists of the echinocandins. These drugs are non-competitive inhibitors of the enzyme 1,3- $\beta$-D glucan synthase, responsible for the synthesis of $\beta-1,3-\mathrm{D}$-glucan, a polysaccharide important in maintaining the structure and integrity of the fungal cell wall as well as cell growth of fungi $(105,106)$. Inhibition of $1,3-\beta-D$ glucan synthase leads to the formation of defective cell walls that prevent the fungal cells from maintaining shape and which lack the rigidity required to tolerate osmotic pressure, thus resulting in cell lysis of yeast (106) and the formation of aberrant hyphae in molds (107).

This class of drugs includes caspofungin and micafungin, which are used clinically against Candida and Aspergillus, but have no activity against Cryptococcus, Trichosporon, Pseudallescheria, Alternaria, Zygomycetes, Fusarium and other fungi $(106,108-111)$. In addition, they are expensive, only available as intravenous formulations, and acquired resistance has been reported (112). 


\section{Clinical Azole Resistance}

The development of resistance to the azoles is a problem of increasing medical significance. The extensive use of fluconazole in the previous two decades has fostered the emergence of several intrinsically resistant Candida species such as C. glabrata and C. krusei (113), and the development of acquired resistance in C. albicans and other fungal species (114-116). In addition, a high level of resistance toward azoles is also well known for C. inconspicua, C. rugosa, and C. norvegensis (117). The overall resistance in clinical isolates of Candida spp. to fluconazole and voriconazole is $\sim 3-6 \%$, and the level of resistance has remained constant over a decade (118). However, a recent report revealed clinical azole resistance in $12.5-18.8 \%$ of $C$. albicans isolates, $10.2-13.6 \%$ of C. tropicalis and up to $25 \%$ of $C$. glabrata. (118). Likewise, triazole resistance in Aspergillus fumigatus is increasingly being recognized with up to $6 \%$ of clinical isolates were resistant (119). Furthermore, concern has been expressed over the possibility of selection for resistance in opportunistic fungi such as Aspergillus spp. in the environment as azole fungicides are widely used in agriculture (120). Hence the development of environmentally azole resistant fungi may represent a source of cross-resistant isolates in the clinics.

\section{Mechanisms of resistance to azole antifungals in Candida albicans}

Microbiological drug resistance can be defined as the reduced susceptibility, or lack thereof, to a particular antifungal during in vitro susceptibility testing observed when the minimum inhibitory concentration (MIC) of such drug surpasses the established susceptibility breakpoint for a fungal species (107). On the other hand, clinical resistance can be defined as the inability to eliminate a fungal infection using an antifungal that is known to possess in vitro and in vivo activity against a particular species (107).

Fungal drug resistance can be intrinsic or acquired. The first is an innate property of a fungal species and occurs without previous exposure to the drug, and it is exemplified by fluconazole resistance in C. krusei and echinocandin resistance in Cryptococcus neoformans. Acquired resistance develops after drug exposure and is exemplified by the development of resistance to azoles in C. albicans (107).

Several acquired mechanisms of azole resistance have been characterized in $C$. albicans and usually appear in a stepwise manner (81). These include overexpression and mutations within the ERG11 gene and overexpression of efflux pumps, such as Cdrlp and Mdr1p. However, a combination of the above mechanisms is usually necessary to confer a significant reduction in azole susceptibility (121), and notably, the resistance of many fungal isolates is not fully accounted for by any of the mechanisms described so far (122). Thus, it is imperative to understand the mechanisms of azole resistance and tolerance in order to devise therapeutic strategies to enhance their efficacy, overcome drug resistance and extend their span of clinical use. Additionally, accurate interpretation of azole susceptibility testing of fungal isolates is a central component of patient management. However, this can be complicated by phenomena such as trailing growth 
$(123,124)$, which is observed in Candida spp. in vitro during standard susceptibility testing. Trailing growth occurs when a clinical isolate appears to be sensitive to azole treatment after 24 hours of drug exposure but appear to be resistant at 48 hours (123). Yet, the molecular basis as well as the clinical and biological relevance of the trailing growth phenomenon remains unclear.

Azole resistance mechanisms described for $C$. albicans can be grouped into three main categories: decreased intracellular drug accumulation, changes in the target enzyme for the drug, and alteration in sterol biosynthesis.

\section{Decreased intracellular drug accumulation}

The main mechanism contributing to reduction in the accumulation of azole antifungals within the fungal cell is increased drug efflux (81) as result of overexpression of efflux pumps that extrude azoles back into the extracellular environment (Figure 1-3). Early studies on these mechanisms of azole resistance in clinical isolates of $C$. albicans highlighted the elevated mRNA expression of members of the ATP-binding cassette (ABC) superfamily and major facilitator superfamily (MFS) (125). The ABC and MFS transporters are membrane-bound proteins that use the energy generated from ATP hydrolysis or the proton gradient, respectively, to move a wide variety of compounds across membranes, including drugs, sugars, and amino acids, among others $(126,127)$. Remarkably, the prevalence of efflux pumps as a mechanism of azole resistance appears to be high; Perea et al (128) found that $85 \%$ of azole resistant isolates evaluated exhibited elevated expression of the genes encoding for efflux pumps.

In C. albicans, overexpression of $C D R 1$ and $C D R 2$ encoding efflux pumps belonging to the $\mathrm{ABC}$ transporter family has been associated with cross-resistance to azoles and amorolfine $(125,129)$. Although more $C D R$ genes have been identified $(81$, $129,130)$, their role in azole resistance is unclear.

It has been shown that increased $C D R 1, C D R 2$ and $M D R 1$ mRNA levels in clinical isolates are the result of increased transcription of their respective genes (130). This is often a consequence of gain of function mutations in the genes encoding the transcription factors Tac1p (131) (which regulates CDR1 and CDR2) and Mrrlp (132) (which regulates $M D R 1$ ) (133), leading to hyperactivity of the transcription factor and the overexpression of the target genes.

\section{Changes in the target enzyme}

The transcription of several genes involved in ergosterol biosynthesis, including $E R G 9, E R G 1, E R G 7, E R G 11, E R G 25$ and $E R G 3$ is upregulated following treatment with azole antifungals in susceptible isolates of $C$. albicans, presumably as a response to depletion of ergosterol and changes in cellular sterol composition $(81,134)$. However, increased expression of ERG11 has been shown to be associated with azole resistance in a number of matched sets of susceptible and resistant clinical isolates (Figure 1-3) (125, 128). Another study showed that $35 \%$ of azole resistant isolates were found to have 


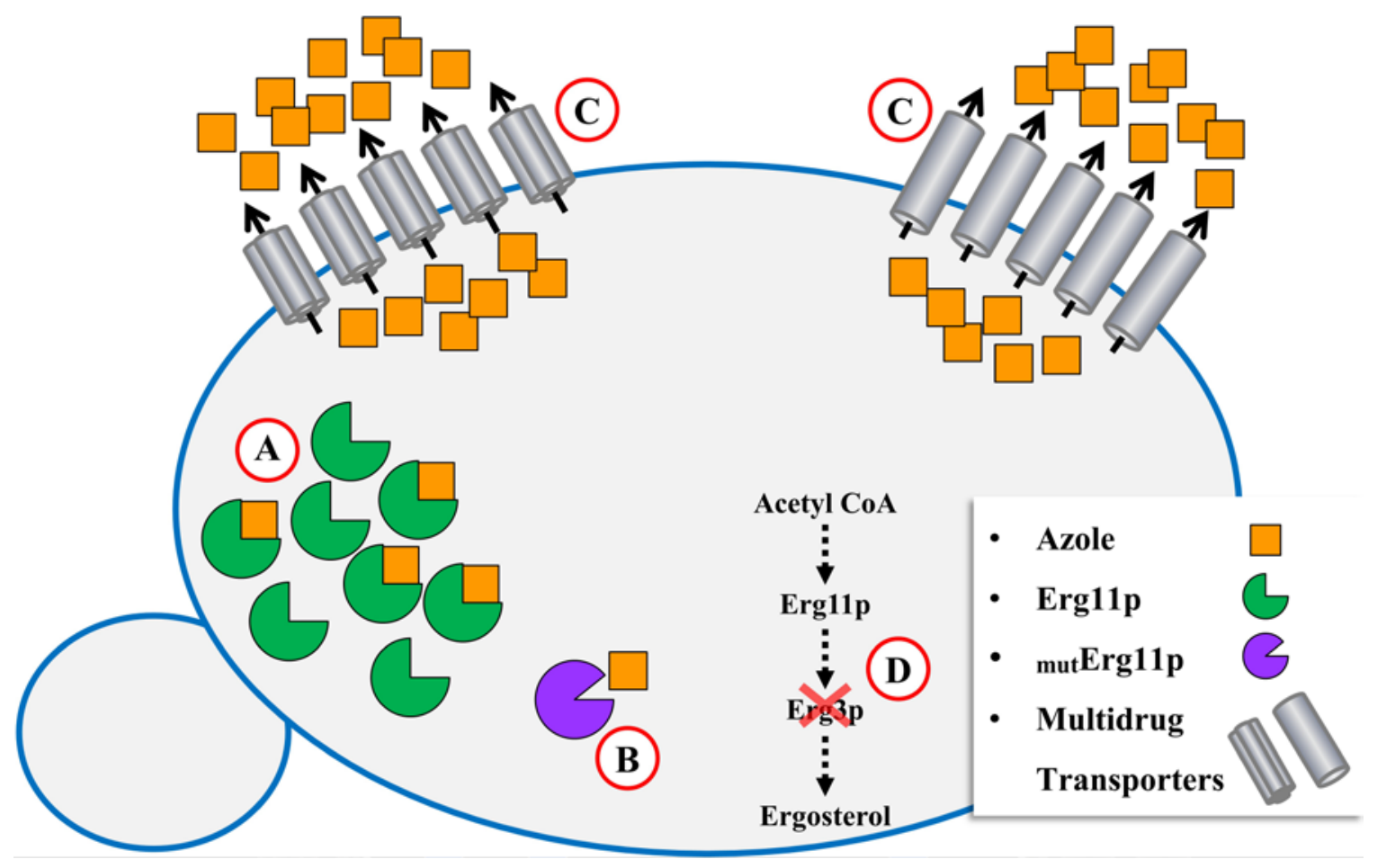

Figure 1-3. Azole resistance mechanisms in fungi.

(A) Several mechanisms that contribute to azole resistance have been identified in pathogenic fungi. (A) Overexpression of the target enzyme lanosterol demethylase (Erg11p) allows for the replacement of azole-inhibited proteins. (B) Mutations within the ERG11 gene lead to amino acid susbtitutions in the enzyme (mutErg11p) that can reduce affinity for the drug. (C) Overexpression of multidrug transporters such as Cdr1p and Mdr1p result in reduced accumulation of the azoles within the fungal cell. (D) Mutations in genes of the ergosterol biosynthetic pathway, such as ERG3, which result in loss of function allows fungi to survive azole exposure by avoiding the production of toxic sterol intermediaries that otherwise accumulate in the plasma membrane. 
elevated ERG11 mRNA levels compared to susceptible isolates (128). The transcription factor Upc2p regulates the expression of ERG1 $(135,136)$. Overexpression and gain of function mutations within $U P C 2$ have been shown to be associated with azole resistance (135). Activating mutations in UPC2 can lead to constitutive overexpression of ERG11 and hence, contribute to azole resistance $(137,138)$.

In addition, point mutations within $E R G 11$ leading to amino acid substitutions around the active site of the enzyme have been linked to azole resistance by reducing the affinity of Erg 11p for the drug (Figure 1-3) (139). Events of gene conversion leading to the selection for allelic variants that confer resistance under selective pressure have been reported $(83,140)$.

\section{Alterations in the sterol biosynthetic pathway}

Azole treatment leads to the accumulation of 14 $\alpha$-methylergosta-8,24(28)-dien$3 \beta, 6 \alpha$-diol, which is believed to be a toxic sterol byproduct of Erg3p activity accompanying Erg11 inhibition and has been directly linked to the antifungal activity of azoles (141). However, certain azole resistant clinical isolates accumulate $14 \alpha-$ methylfecosterol following azole treatment instead of the toxic sterol, suggesting loss of Erg3p function (142). This alternative sterol allows C. albicans to overcome growth inhibition otherwise caused by azoles. Thus, mutations that abolish Erg3p activity are regarded as a mechanism of azole resistance (Figure 1-3) (142).

\section{Fungal azole tolerance}

The static nature of the azoles allows for fungal cells to persist even at high concentrations of the drugs. Azole tolerance is defined as the persistent growth of a fungal strain at concentrations above the MIC. A well-known example of azole tolerance is the trailing growth phenomenon that is observed during azole susceptibility testing of C. albicans isolates. Trailing isolates appear to be susceptible to azole treatment in vitro at 24 hours; however, by the 48 hour time-point, they appear to be resistant to azole treatment (143). This phenotype is commonly observed when evaluating susceptibility to triazole drugs such as fluconazole and itraconazole (123). However, experiments performed in mouse models of disseminated infection indicate that $C$. albicans trailing isolates are susceptible to azole treatment in vivo (143-145).

Several mechanisms have been linked to azole tolerance in fungi. The best studied mechanisms of azole tolerance are related to calcium dependent signaling, mainly through the calcineurin signaling pathway $(146,147)$. Calcineurin is a $\mathrm{Ca}^{2+}$ and calmodulin dependent serine/threonine protein phosphatase that links $\mathrm{Ca}^{2+}$-dependent signaling to a multitude of cellular processes (148). Pharmacological or genetic inhibition of calcineurin signaling sensitizes several pathogenic fungi to azole exposure $(147,149)$. For example, combination of fluconazole and cyclosporine A (a cyclophilin inhibitor) or FK506 (a FKBP inhibitor) has synergistic effects in that addition of CsA or FK506 enhance the antifungal efficacy of fluconazole $(149,150)$. Moreover, deletion of genes 
encoding for upstream and downstream elements of the calcineurin signaling pathway, such as the transcription factor Crzlp $(151,152)$ or the chaperone Hsp90p (153), increases fungal killing by azole treatment. Furthermore, several proteins involved in maintenance of calcium homeostasis have been shown to be required for the survival of several fungi to azole treatment, as deletion of the genes encoding for such proteins enhance the antifungal effects of the azoles (146). These include the plasma membrane calcium channel formed by Cch1p and Mid1p (154).

\section{Vacuolar Function in Candida albicans}

Within the fungal cell, the vacuole is one of the largest organelles (155). While the functions of this acidic membrane-bound compartment have been defined using the model $S$. cerevisiae $(49,156)$, the findings have been extrapolated to pathogenic fungi and is used as a model for the mammalian lysosome. These functions include degradation of macromolecules for the recycling of their building blocks, metabolite storage, stress tolerance, ion homeostasis and response to starvation in yeast (156).

An important feature of the vacuole is that many of its functions depend on maintaining an acidic lumen (157), a task that depends on the activity of the vacuolar $\mathrm{H}^{+}$ATPase (V-ATPase), an enzyme complex responsible for the establishment of a proton gradient across the vacuolar membrane driven by ATP hydrolysis (158). For example, amino acid storage within the vacuole relies on the proton gradient and activity of at least eight $\mathrm{H}^{+}$/amino acid exchangers in the vacuolar membrane (156). The main amino acids found to be stored in the vacuole through this system are arginine, lysine, histidine, phenylalanine, tryptophan, tyrosine, glutamine, asparagine, isoleucine and leucine (156).

The vacuole is also the major site of storage of $\mathrm{Ca}^{2+}$ in the fungal cell. $\mathrm{Ca}^{2+}$ dependent signaling requires the maintenance of low levels of this ion in the cytosol under resting conditions (159). The initiation of $\mathrm{Ca}^{2+}$ signals entail its release from intracellular stores and influx from the extracellular media in response to different stimuli, followed by efflux from the cell and storage back into organelles until resting levels are achieved (160). Importantly, several $\mathrm{Ca}^{2+}$ pumps and channels involved in this process are located on the vacuolar membrane and include the pump Pmclp, the $\mathrm{Ca}^{2+} / \mathrm{H}^{+}$ exchanger Vcxlp and the channel Yvclp $(160,161)$.

Furthermore, the vacuole works as a site of storage for polyphosphate. This macromolecular anion serves in amino acid and cation retention. It is also important for storage of inorganic phosphate and $\mathrm{pH}$ regulation (156). Remarkably, more than $90 \%$ of cellular $\mathrm{Ca}^{2+}$ is associated with large chains of polyphosphate inside the vacuole (161).

The vacuole also functions as a storage of alkali metals such as $\mathrm{K}^{+}$and $\mathrm{Na}^{+}$and it is important to maintain a high $\mathrm{K}^{+} / \mathrm{Na}^{+}$ratio in the cell (159). This process also depends on the proton gradient created by the V-ATPase and the activity of the cation/ $\mathrm{H}^{+}$ antiporter Vnx1p (162). The vacuole is also important for detoxification of metal ions. Accumulation of ions such as $\mathrm{Cd}^{2+}, \mathrm{Co}^{2+}$ and $\mathrm{Cu}^{2+}$ at high concentrations in the 
cytoplasm is toxic, but they can be sequestered in the vacuole using a proton gradient dependent mechanism $(163,164)$. On the other hand, $\mathrm{Fe}^{2+}$ can also be sequestered in the vacuole via a mechanism independent of vacuole acidification (165).

Mutants of S. cerevisiae and C. albicans with severe vacuolar dysfunction are hyper-susceptible to osmotic stress (156). This includes mutants with defects in protein sorting, polyphosphate formation, acidification, vacuole biogenesis, ion regulation, and homeostasis of several amino acids (156).

In C. albicans and other pathogenic fungi, the vacuole plays a pivotal role in pathogenesis. Vacuolar defects result in several pathogenesis related phenotypes, including susceptibility to a wide variety of stresses and loss of virulence-associated traits $(49,51,166)$. Vacuolar expansion has been shown to be important for the migration of cellular material during hyphal formation, while regulation of vacuolar fragmentation and fusion help control cell size and branching $(167,168)$. Moreover, C. albicans mutants with severe vacuolar defects have reduced rates of planktonic growth (49), have reduced capacity to form hyphae and are unable to kill phagocytes, a virulence attribute important during invasion of host tissue and escape from immune cells $(49,166)$. In addition, several mutants with altered vacuolar function fail to cause disease in a mouse model of disseminated candidiasis $(51,166)$. Mutants severely affected in vacuole biogenesis are not only unable to kill systemically infected mice but are also rapidly cleared from the target organs (51).

Recent evidence points to an important role of ergosterol in vacuolar function. Ergosterol has been shown to be required for endocytic trafficking to the vacuole (169) and for the priming step during homotypic vacuole-vacuole fusion in S. cerevisiae (170). In addition, several non-essential $S$. cerevisiae ergosterol biosynthetic mutants have a fragmented vacuole morphology $(170,171)$. Furthermore, C. albicans mutants lacking Erg2p or Erg24p have impaired vacuolar function and were avirulent in a mouse model of disseminated candidiasis (172). Yet, the consequences of treatment with ergosterol biosynthesis inhibitors of clinical importance upon vacuolar function and the contribution of vacuole dysfunction to their antifungal activity remain to be fully understood.

\section{Research Hypothesis}

The membrane lipid ergosterol is the most common target of several classes of antifungals. It has been widely assumed that both the depletion of cellular ergosterol and the accumulation of 'toxic' sterol intermediates in the plasma membrane account for the antifungal activity of ergosterol biosynthesis inhibitors (92). Nonetheless, the cellular pathways affected by ergosterol depletion remain poorly understood (87). Moreover, it remains unknown whether the antifungal activity of the azoles is only due to plasma membrane dysfunction, or if it is compounded by the dysfunction of other intracellular membranes. 
Although the bulk of cellular ergosterol is found in the plasma membrane, other intracellular membrane compartments such as the fungal vacuole also contain ergosterol $(87,173)$. Thus it is likely that the function of other intracellular membranes is profoundly impacted by ergosterol availability following treatment with ergosterol biosynthesis inhibitors.

In a high-throughput screen for compounds that target the fungal vacuole, several azole antifungals were identified as causing disruption of this organelle in C. albicans (unpublished results). After considering the importance of the vacuole in C. albicans biology, I hypothesize that disruption of vacuolar integrity contributes to the antifungal activity of the azoles and, in turn, vacuolar dysfunction can impact the antifungal efficacy of this class of drugs.

\section{Research Objectives}

In order to address the research hypothesis, I have established 4 research objectives:

- To characterize the effects of azole treatment upon the integrity of the C. albicans vacuole (Chapter 2).

- To determine if membrane trafficking to the vacuole affects susceptibility to azole antifungals (Chapter 3).

- To determine the mechanisms by which a C. albicans mutant lacking the Rab GTPase Vps21p is tolerant to azole antifungals (Chapter 4).

- To study the interactions between endosomal trafficking and known mechanisms of azole resistance (Chapter 5). 


\section{CHAPTER 2. THE AZOLE ANTIFUNGALS SEVERELY DISRUPT VACUOLAR INTEGRITY IN CANDIDA ALBICANS THROUGH INHIBITION OF ERG11P*}

\section{Introduction}

Several of the most successful antifungal therapies currently used in both the clinical and agriculture settings target the biosynthesis of the membrane lipid, ergosterol. Azoles are the most important class of antifungals; they inhibit ergosterol biosynthesis by preventing the function of lanosterol-14 $\alpha$-demethylase (Erg11p), which in turn causes plasma membrane damage, presumably as a consequence of both ergosterol depletion and the accumulation of toxic sterol intermediaries. Ergosterol is the major sterol in fungal cell membranes, and plays an essential role in maintaining and regulating plasma membrane integrity, fluidity and permeability. In addition, ergosterol is also required for the normal function of a number of plasma membrane proteins, including several proteins involved in nutrient uptake at the plasma membrane such as the tryptophan permease Tat $2 p(82)$ and the amino acid permease Gap1 (174), the activity of chitin synthase (175, 176), and the association of beta-1,3-glucanosyltransferase Gas 1p and Pmalp with lipid rafts (177). Accordingly, ergosterol is required for several biologically important cellular functions including cellular signaling (178), sporulation in S. cerevisiae (179), as well as polarized hyphal growth in C. albicans (180).

The bulk of cellular ergosterol is found in the plasma membrane. As such, it is widely assumed that the primary consequence of ergosterol depletion, and the accumulation of sterol-diol following azole treatment is plasma membrane dysfunction. However, other intracellular membrane compartments like the mitochondria, endoplasmic reticulum, trans-Golgi network and the fungal vacuole, are also known to contain smaller amounts of ergosterol $(87,173)$. Thus it is likely that the function of other intracellular membranes is profoundly impacted by ergosterol availability following treatment with ergosterol biosynthesis inhibitors. For example, inhibition of ergosterol biosynthesis can severely compromise mitochondrial function (181). However, the consequences of azole treatment upon the function and integrity of intracellular membranes and organelles are not well characterized.

A recent high-throughput screen conducted in our laboratory identified several imidazole antifungals as disrupting vacuolar integrity in C. albicans. Previous studies have indicated that ergosterol is required for endocytic trafficking from the plasma membrane to the fungal vacuole (95), and to support homotypic vacuole-vacuole fusion

* Modified by permission of American Society for Microbiology. Luna-Tapia A, Kerns ME, Eberle KE, Jursic BS, Palmer GE. 2015. Trafficking through the late endosome significantly impacts Candida albicans tolerance of the azole antifungals. Antimicrob Agents Chemother 59:2410-2420. doi:10.1128/AAC.04239-14. 
in an in vitro biochemical assay. In addition, ergosterol biosynthetic (erg) mutants of Sacharomyces cerevisiae exhibit defects in vacuolar fusion and are known to have aberrant vacuole morphologies $(96,97)$. Furthermore, it has recently been proposed that ergosterol depletion following azole treatment impairs the function of the V-ATPase (79) responsible for vacuolar acidification, which in turn is required for many processes in this organelle. This is of significance as defects in vacuolar function and acidification substantially diminish C. albicans capacity to endure physiological stress, produce tissue invasive hyphae, and cause lethal infections in mice (49, 51, 166, 182-184). Furthermore, severe defects in vacuolar biogenesis can severely impair $C$. albicans growth rates (49). However, it remains unknown whether azole-induced vacuolar disruption contributes to the overall antifungal activity of the azoles, and in turn, how defective vacuolar function may influence susceptibility to the azoles. Thus, the objective of this chapter is to characterize the effects of azole treatment upon the integrity of the C. albicans vacuole.

\section{Materials and Methods}

\section{Growth and media conditions}

All C. albicans strains were routinely grown on yeast extract-peptone-dextrose (YPD) agar plates or in liquid medium at $30^{\circ} \mathrm{C}$ and supplemented with uridine (50 $\mu \mathrm{g} / \mathrm{mL}$ ) when necessary. Transformant selection was carried out on minimal YNB medium ( $6.75 \mathrm{~g} / \mathrm{L}$ yeast nitrogen base without amino acids, $2 \%$ dextrose, $2 \%$ Bacto agar), supplemented with the appropriate auxotrophic requirements as described for $S$. cerevisiae (185) or uridine at $50 \mu \mathrm{g} / \mathrm{mL}$. All C. albicans strains were stored frozen at $80^{\circ} \mathrm{C}$ in $20 \%$ glycerol.

For growth curves, overnight cultures in YPD at $30^{\circ} \mathrm{C}$ were sub-cultured into 15 $\mathrm{mL}$ fresh YNB medium at an initial cell concentration of $1 \times 10^{6}$ cells $/ \mathrm{mL}$ in the presence of $1 \mu \mathrm{g} / \mathrm{mL}$ fluconazole or $0.5 \%$ dimethyl sulfoxide (DMSO - drug free control), and incubated at $30^{\circ} \mathrm{C}$ with shaking. Samples were taken every 30 minutes and optical density was determined spectroscopically at $600 \mathrm{~nm}$.

\section{Plasmid construction}

All oligonucleotides used in this study are listed in Table 2-1. Plasmid pKE1:GFP-YPT72 (186) for expression of the GFP-Ypt72 fusion protein to label the vacuolar membrane was made by amplifying the coding sequence of the previously described GFP-YPT72 gene fusion (51) with primer set GFPSALIF + YPT72ORFRMluI, and cloning the product downstream of the $A C T 1$ promoter, between the SalI and $M l u \mathrm{I}$ sites of the pKE1 expression vector (187). Plasmid pKE1:CPP-GFP was constructed as follows. The coding sequence of the predicted C. albicans Cpylp prepropetide (codons 1-129) (188), was amplified by PCR from SC5314 gDNA using the primers CPPORFFSalI and CPPORFR-EagI, and cloned between the SalI and EagI restriction 
Table 2-1. List of oligonucleotides used in the study described in Chapter 2.

\begin{tabular}{|c|c|}
\hline Primer & Sequence (5'-3') \\
\hline ARG4DETF & ATCAATTAACACAGAGATACC \\
\hline ARG4DETR & CCGAGCTTGGCGTAATCATGG \\
\hline ARG4INTF2 & AAGCTAGTGTGGAAAGAAGAG \\
\hline ARG4INTR2 & AATGACTGAATTATGTCGGTC \\
\hline CPPORFF-SaII & TCAGTCGACATGAAGTTATCAAAATCCAC \\
\hline CPPORFR-EagI & TCAGGGCCGACCACCACCACCTTTAATTC \\
\hline ERG11AMPF2-KpnI & TCAGGTACCAATGAAAATGTCGTGGGGAGC \\
\hline ERG11DISF & $\begin{array}{l}\text { TTCTTTCCATATTACTTGTCTTCTTTTTATTATATATATAAG } \\
\text { TTTCTTTTCAAGAAGATCATAACTCAATGTTTTCCCAGTC } \\
\text { ACGACGTT }\end{array}$ \\
\hline ERG11DISR & $\begin{array}{l}\text { TGTGTTAATCCAACTAAGTAACAAAATGAAAACAATCTG } \\
\text { AACACTGAATCGAAAGAAAGTTGCCGTTTTATGTGGAAT } \\
\text { TGTGAGCGGATA }\end{array}$ \\
\hline ERG11F-KpnI-SaII & TCAGGTACCGTCGACGCCAACAGAATTACTACCCAA \\
\hline ERG11ORFF & TCAGTCGACATGGCTATTGTTGAAACTGTC \\
\hline ERG11ORFR & TCAACGCGTTGAATCGAAAGAAAGTTGCCG \\
\hline ERG11R-KpnI-MluI & TCAGGTACCACGCGTTTGGGTAGTAATTCTGTTGGC \\
\hline ERG11SEQR1 & $\begin{array}{l}\text { TTTTCATAACATTGGCAACCC } \\
\text { AAATCAATTTTTATATATAAATAGACAAAGAAAGGGAAT }\end{array}$ \\
\hline ERG11TETOF & $\begin{array}{l}\text { TCAATCGTTATTCTTTCCATATTACTTGTCGTAATACGACT } \\
\text { CACTATAGGG } \\
\text { ATCTGTTGTGTAACACTAAGGGACAAAAAATAATTAATG }\end{array}$ \\
\hline ERG11TETOR & $\begin{array}{l}\text { CCATCAATGACAGTTTCAACAATAGCCATCTAGTTTTCT } \\
\text { GAGATAAAGCTG }\end{array}$ \\
\hline ERG3AMPF-SacI & TCAGAGCTCGACTACGCGAGACCACACTTGC \\
\hline ERG3AMPR-SacI & TCAGAGCTCGTGAAATTACTTACAATATGGAG \\
\hline ERG3DETF & GCTGATGTTTTCCCTAAAGATGG \\
\hline ERG3DETR & GTAAGAGTTACCAAGTCTATCCC \\
\hline ERG3DISF & $\begin{array}{l}\text { CCCTTCCCATTTCTTTCCCTATTGTGCATATAAGTTCAATC } \\
\text { TTTTTTTCTTTCTTTCGGATTCGGTTTAGTGTGGAATTGT } \\
\text { GAGCGGATA }\end{array}$ \\
\hline ERG3DISR & $\begin{array}{l}\text { GGAAAAATAGTCAATGGTCCAAAACAAAGATGTACCAA } \\
\text { TCATTGTTCAACATATTCTCTATCGTCAACTTGTTTTCCC } \\
\text { AGTCACGACGTT }\end{array}$ \\
\hline GFPAMP-MluI & TCAACGCGTTTATTTGTACAATTCATCC \\
\hline GFPEAGF & TCACGGCCGATGTCTAAAGGTGAAGAATTATTC \\
\hline GFPSALIF & TCAGTCGACATGTCTAAAGGTGAAGAATTATTC \\
\hline HIS1DETF & TCATCCTCCAGGATCCCGCGG \\
\hline HIS1DETR & TGTCACCTAAATAGCTTGGCG \\
\hline HIS1INTF2 & ACTGTATCCTCTTCTGTCCCC \\
\hline HIS1INTR2 & CGACCATATGGGAGAGCTCCC \\
\hline
\end{tabular}


Table 2-1. (Continued).

\begin{tabular}{ll}
\hline \multicolumn{1}{c}{ Primer } & \multicolumn{1}{c}{ Sequence (5'-3') } \\
\hline LUXINTDETF & CTGACCTTTAGTCTTTCCTGC \\
LUXINTDETR & CAGTAGTACTTGTTGTTGTATCG \\
TETODETF & GTTGACACTTCTAAATAAGCG \\
URA3DISF1 & ATGACAGTCAACACTAAGACC \\
URA3DISF2 & CCACTAAGGAATTCCTTGAATCAAGAAGAAGGATTTGAT \\
URA3DISR2 & TGGCT \\
URA3DISR3-SacII & CAAATAAGCATTCCAACCAGC \\
URA3INTF & TCACCGCGGCAATATGAGTCTTGATTAAGC \\
URA3INTF2 & TTATACCATCCAAATCCCGCG \\
URA3INTR & CAATTATAAATGTGAAGGGGG \\
YPT72ORFR-MluI & TCAACGCGTTTTGTTCTCTTTCTTCACCTG \\
\hline
\end{tabular}


sites of pKE1 (187). A C. albicans codon-optimized GFP coding sequence was then amplified by PCR from plasmid pGFPURA3 (189) using primers GFPEAGF and GFPAMPR-MluI, and cloned in-frame and downstream of the CPY1 prepropeptide coding sequence, between EagI and MluI sites to yield the CPP-GFP fusion construct.

Plasmid pGUD1 was constructed as follows. A $210 \mathrm{bp}$ portion of the 3' end of the URA3 ORF was amplified from pGEM-URA3 (190) with primer set URA3DISF2 + URA3DISR2, and cloned into the vector pGEM ${ }^{\circledR}$-T Easy (Promega). A 193 bp portion of the 5' end of the URA3 ORF was then amplified using the primer set URA3DISF1 and URA3DISR3-SacII. This product was then digested with SphI and SacII and cloned between the same sites of the pGEM ${ }^{\circledR}-T$ Easy vector, adjacent to the 3 ' end of the URA3 gene. This produced an ura 3 allele lacking a $390 \mathrm{bp}$ central portion of the ORF.

\section{C. albicans strain construction}

Strains used in this study are listed in Table 2-2. Gene deletion strains were constructed by the PCR-based approach described by Wilson et al., (190), using the ura $3 \Delta / \Delta$ his $1 \Delta / \Delta \arg 4 \Delta / \Delta$ strain BWP17 (kindly provided by Dr. Aaron Mitchell, Carnegie Mellon University).

$E R G 3$ deletion cassettes were amplified by PCR with primer pair ERG3DISF + ERG3DISR, using pRS-ARG4 $\triangle$ SpeI or pGEM-HIS1 (190) as templates. Each ERG3 allele was sequentially deleted from strain BWP17 using ARG4 and HIS1 markers to generate $\operatorname{erg} 3 \Delta / \Delta u r a 3 \Delta / \Delta$ gene deletion mutants. Correct integration of deletion cassettes was confirmed at each step by PCR with the following primer sets: ARG4INTF2 + ERG3AMPR-SacI and ARG4INTR2 + ERG3AMPF-SacI (ARG4 integration), or HIS1INTF2 + ERG3AMPR-SacI and HIS1INTR2 + ERG3AMPF-SacI (HIS1 integration). The lack of an intact $E R G 3$ allele was confirmed using primer pair ERG3DETF and ERG3DETR.

Strain THE1 (191) was kindly provided by H. Nakayama and M. Arisawa (Nippon Roche). One allele of ERG11 was deleted using the recyclable URA3-dpl200 selection marker, which was amplified from pDDB57 with primer pair ERG11DISF + ERG11DISR. Correct integration was then confirmed by PCR using primer pair URA3INTR + ERG11R-KpnI-MluI. The resulting strain was then made ura3- through 5fluoroorotic acid (5'FOA) selection on YNB medium supplemented with uridine and 1 $\mu \mathrm{g} / \mathrm{mL} 5^{\prime}$ FOA (192) in order to isolate $u r a 3^{-}$segregants that had excised the URA3 marker gene. Excision of the $U R A 3$ gene was confirmed using the flanking primer set ERG11AMPF2 + ERG11R-KpnI-MluI (1307 bp product) (186).

The native 5' UTR of the second ERG11 allele was then replaced with a URA3tet $O$ promoter cassette which was amplified from plasmid p97CAU (191), using ERG11TETOF + ERG11TETOR primers. Correct promoter replacement was confirmed using primer pair TETODETF + ERG11SEQR1 to confirm integration of the tet $O$ 
Table 2-2. List of strains used in the study described in Chapter 2.

\begin{tabular}{|c|c|c|}
\hline Strain & Relevant Genotype & Reference \\
\hline CAI4 & ura3 $\Delta / \Delta$ & $(193)$ \\
\hline BWP17 & $\operatorname{ura} 3 \Delta / \Delta$ his $1 \Delta / \Delta \arg 4 \Delta / \Delta$ & $(190)$ \\
\hline THE1 & ura $3 \Delta / \Delta$ ade $2 \Delta / \Delta E N O 1 / \Delta: E N O 1$-tetR-ScHAP4AD-3× HA-ADE2 & $(191)$ \\
\hline CAI4 + GFP-YPT72 & ura3 $\Delta / \Delta: U R A 3: G F P-Y P T 72$ & This study \\
\hline CAI4 + CPP-GFP & ura3A/A:URA3:CPP-GFP & This study \\
\hline $\operatorname{erg} 3 \Delta / \Delta+$ GFP-YPT72 & ura3 $/ \Delta: U R A 3: G F P-Y P T 72$ his $1 \Delta / \Delta \arg 4 \Delta / \Delta \operatorname{erg} 3 \Delta: H I S 1 / \operatorname{erg} 3 \Delta: A R G 4$ & This study \\
\hline $\operatorname{erg} 11 \Delta / E R G 11+$ GFP-YPT72 & 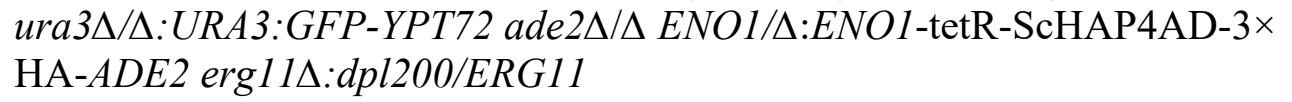 & This study \\
\hline $\operatorname{erg} 11 \Delta /$ tetO-ERG11 + GFP-YPT72 & $\begin{array}{l}\text { ura3 } \Delta / \Delta: U R A 3: G F P-Y P T 72 \text { ade } 2 \Delta / \triangle E N O 1 / \Delta: E N O 1: \text { tetR-ScHAP4AD-3× } \\
\text { HA-ADE2 erg11 } 1 \Delta: \text { dpl200/tetO-ERG11 }\end{array}$ & This study \\
\hline
\end{tabular}


promoter upstream of the remaining ERG11 ORF, and ERG11AMPF2-KpnI + ERG11SEQR1 to confirm the absence of a native ERG11 allele (186).

Next the $\operatorname{erg} 11 \Delta /$ tetO-ERG11 strain was transformed with a URA3 deletion cassette that was amplified from plasmid pGUD1 with primer pair URA3DISF1 + URA3DISR2. This consisted of the URA3 ORF lacking a $390 \mathrm{bp}$ internal sequence. Transformants were then selected on 5'FOA plates (see above), and correct disruption of the URA3 ORF confirmed using primer pair URA3DISF1 + URA3DISR2 that flank the internal deletion sequence of URA3. The absence of an intact URA3 ORF (792 bp), and presence of a truncated product (402 bp) confirmed that the URA3 selection marker had been successfully disrupted (186).

Plasmid pKE1:GFP-YPT72 linearized with NheI was introduced into the $\mathrm{ura3}^{-} \mathrm{C}$. albicans strain CAI4 (193), erg $3 \Delta / \Delta$ ura $3 \Delta / \Delta$ and $\operatorname{erg} 11 \Delta /$ tetO-ERG1 lura3 $\Delta / \Delta$, and transformants selected on YNB medium lacking uridine. For construction of the CPPGFP expressing strain of C. albicans, the pKE1:CPP-GFP expression plasmid was linearized with $N h e I$, transformed into strain CAI4, and $U R A 3^{+}$transformants selected. Correct integration of either expression construct fully restores the URA3 and adjacent IROI loci, and this was confirmed by PCR analysis using primer pair LUXINTDETF + LUXINTDETR which yields a 2199 bp product following restoration of the ura3 locus.

\section{Fluorescence microscopy to determine vacuole morphology construction}

Vacuole morphology of C. albicans strains was determined using a GFP-Ypt72p or CPP-GFP fusion protein expressed from pKE1. Cells were observed with an Olympus BX51 fluorescence microscope with a 100X objective and a fluorescein isothiocyanate (FITC) filter set to detect GFP fluorescence. For each field matching phase contrast and FITC fluorescence images were acquired using a QImaging MicroPublisher 3 RTV camera (QImaging) and the CellSens ${ }^{\circledR}$ Digital Imaging Software (Olympus).

Adjustments in the brightness and/or contrast of images were made using the CellSens $\mathbb{R}$ Digital Imaging software. All images presented for a given time point/condition of each experiment were scaled identically to permit direct comparison. In some instances, imaging was performed using a Cytation 5 ${ }^{\mathrm{TM}}$ Cell Imaging Multi-Mode Reader (BioTek).

\section{Results}

\section{Azole antifungals cause severe vacuolar disruption in $C$. albicans}

During a high-throughput screen, we recently identified a variety of imidazole antifungals, including miconazole and ketoconazole, as causing disruption of C. albicans vacuolar integrity (unpublished results). This was further confirmed by fluorescence microscopy using a strain expressing the GFP-tagged GTPase Ypt72p which localizes to the surface of $C$. albicans vacuole (51). As shown in Figure 2-1A, treatment with 
A.
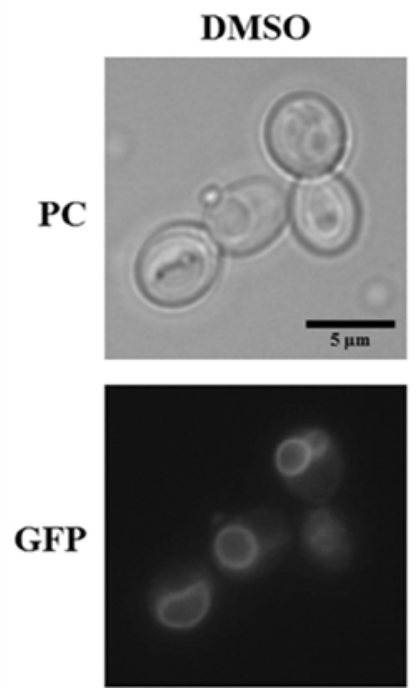

MCZ
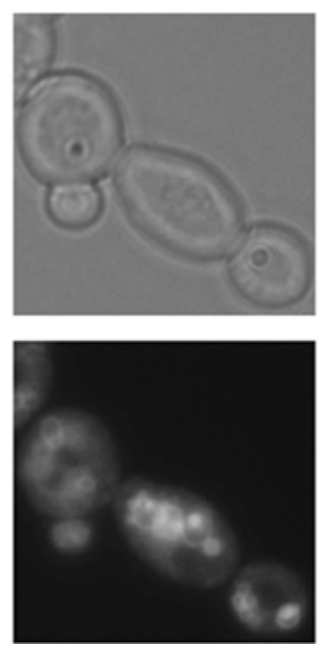

B.
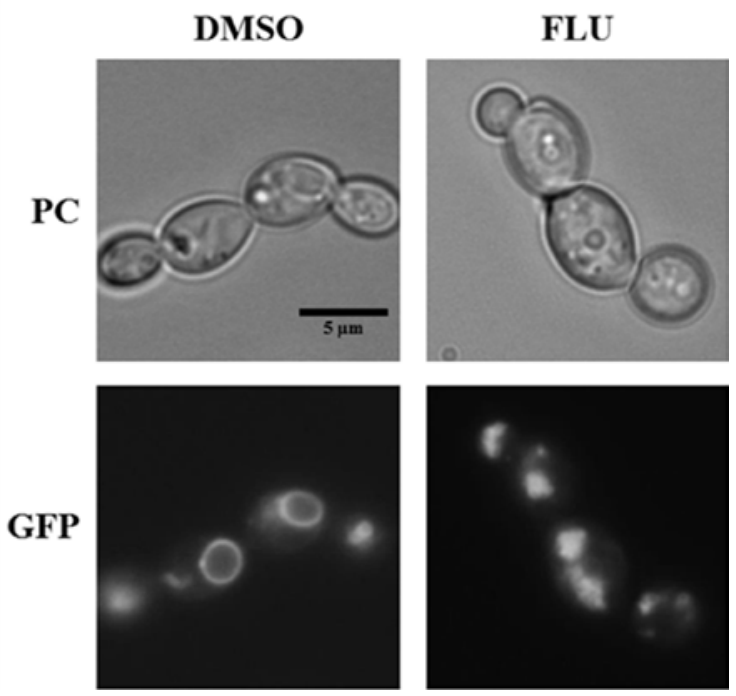

Figure 2-1. Azole antifungals cause severe disruption of the $C$. albicans vacuole. A C. albicans strain expressing GFP-Ypt72p was treated with (A) $1.5 \mu \mathrm{g} / \mathrm{mL}$ of miconazole (MCZ), (B) $1 \mu \mathrm{g} / \mathrm{mL}$ of fluconazole (FLU) or $0.5 \%$ DMSO in YNB and grown at $30^{\circ} \mathrm{C}$ for 4 and 6 hours, respectively. Imaging of vacuolar integrity was performed by fluorescence microscopy using a FITC filter set to detect GFP fluorescence. PC: phase contrast. 
miconazole caused severe disruption of vacuolar integrity, further confirming the results obtained in the chemical screen.

Several imidazoles have been proposed to possess secondary antifungal mechanisms that account for their fungicidal activity at high concentrations, which are independent of their inhibition of ergosterol biosynthesis $(85,86,194,195)$; however, these mechanisms have not been described for the triazoles. We therefore tested if the fungistatic triazole fluconazole, caused similar perturbation of the C. albicans vacuole. Using the $C$. albicans strain expressing GFP-Ypt72p fusion protein, we determined that treatment with fluconazole caused similar disruption of vacuolar integrity as did miconazole (Figure 2-1B). A similar observation was made when the vacuoles were prelabeled with the lipophilic dye FM4-64, which is endocytosed and accumulates within the vacuolar membrane (Figure 2-2). This indicates that both imidazole and triazoles share a common mechanism leading to C. albicans vacuole disruption, presumably Erg11p inhibition.

\section{Vacuole disruption caused by fluconazole treatment occurs before growth inhibition is observed}

In order to determine the sequence of events leading to vacuole disruption following azole treatment, we performed a time-course experiment. The $C$. albicans GFP-Ypt72p tagged strain was sub-cultured into YNB medium $\pm 1 \mu \mathrm{g} / \mathrm{mL}$ fluconazole, and growth, cell viability as well as vacuolar morphology compared at 30 minute intervals. When growth was measured as $\mathrm{OD}_{600 \mathrm{~nm}}$, statistically significant growth inhibition was initially observed in the presence of fluconazole at the 420 minutes time point (Figure 2-3A). However, when cell viability was compared in the same cultures as colony forming units (CFU), fluconazole was found to significantly reduce CFU's vs. the drug free control at the 330 minute time point (Figure 2-3C). In the presence of fluconazole, significant vacuolar fragmentation was initially observed at the 240 minute time point and is pronounced by 270 minutes (Figure. 2-3B). This precedes the reduction in $\mathrm{CFU}$ and the effect on $\mathrm{OD}_{600 \mathrm{~nm}}$, suggesting that vacuolar degeneration is an early event following azole treatment and not merely a secondary consequence of a prolonged growth arrest. Similar observations were also made using a C. albicans strain expressing a GFP tagged version of Carboxypeptidase Y (Figure 2-4), which labels the vacuolar lumen.

\section{Disruption of vacuolar integrity occurs upon inhibition of Erg11p}

In order to confirm that the observed vacuolar defects were a direct consequence of Erg11p inhibition, rather than an 'off-target' effect of the azoles, we constructed a strain in which the transcription of the ERG11 gene could be shut-down using a doxycycline repressible promoter. In the presence of doxycycline, the tetO-ERG11 strain showed similar degeneration of GFP-Ypt72p labeled compartments to those observed following fluconazole treatment (Figure 2-3D). This confirmed that the vacuolar defects 


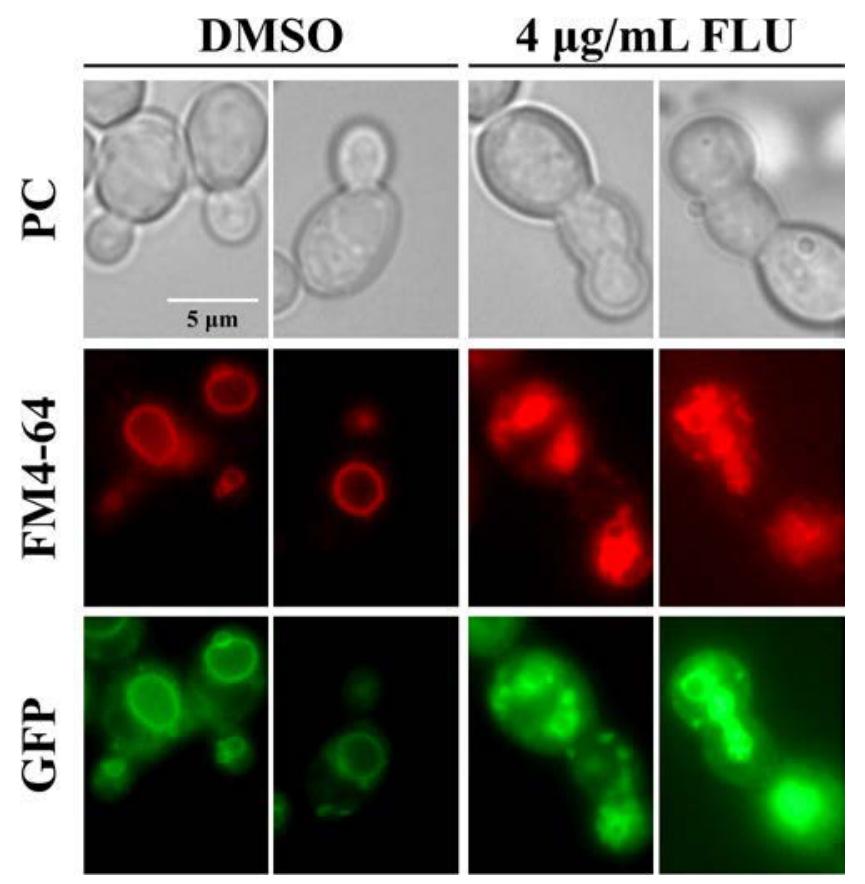

Figure 2-2. The integrity of the $C$. albicans vacuole is severely compromised after azole treatment.

The vacuole of a $C$. albicans strain expressing the GFP-Ypt72p fusion protein was labeled with FM4-64 in YPD medium for 30 minutes. Cells were then sub-cultured into fresh YNB medium $\pm 4 \mu \mathrm{g} / \mathrm{mL}$ of fluconazole (FLU) at $1 \times 10^{6}$ cells $/ \mathrm{mL}$ and grown at $30^{\circ} \mathrm{C}$ for 4 hours. Imaging of vacuolar integrity was performed by fluorescence microscopy using a FITC filter set to detect GFP fluorescence or TRITC for FM4-64 detection. 
Figure 2-3. Inhibition of Erg11p causes vacuolar fragmentation in $C$. albicans. (A, B and C) A C. albicans strain expressing the GFP-Ypt72p fusion protein was subcultured into YNB broth supplemented with either $1 \mu \mathrm{g} / \mathrm{mL}$ fluconazole or $0.5 \%$ DMSO (minus drug control) at $\sim 1 \times 10^{6}$ cells $/ \mathrm{mL}$ and incubated at $30^{\circ} \mathrm{C}$ with shaking. Samples were taken at 30 minutes intervals and growth measured as $\mathrm{OD}_{600 \mathrm{~nm}}(\mathrm{~A})$ and cell viability as colony forming units (CFU). Vacuolar integrity was also observed by fluorescence microscopy using a FITC filter set (C) and matching phase contrast (PC) images also obtained. Data in panels (A) and (B) are the mean and standard deviation of two independent experiments, and the images in panel (C) are representative of each time point in the same two experiments. Growth and CFU's were compared \pm fluconazole for each time point using a two tailed $t$-test. ${ }^{*} P<0.05 ; \S P<0.001$. (D) The $C$. albicans $E R G 11$ gene was placed under the transcriptional control of a doxycycline repressible promoter and the GFP-Ypt72p expression construct introduced into the tetO-ERG11 strain. The tetO-ERG11 strain was then sub-cultured into YNB medium $\pm 5 \mu \mathrm{g} / \mathrm{mL}$ of doxycycline (Dox) at $1 \times 10^{5}$ cells $/ \mathrm{mL}$ and incubated at $30^{\circ} \mathrm{C}$ with shaking. After 8 hours vacuole morphology was observed as described above. 

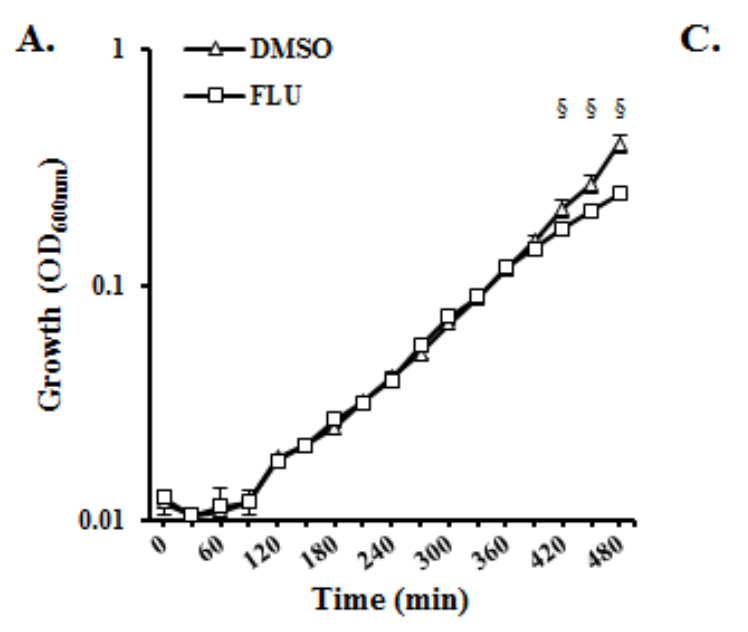

B.
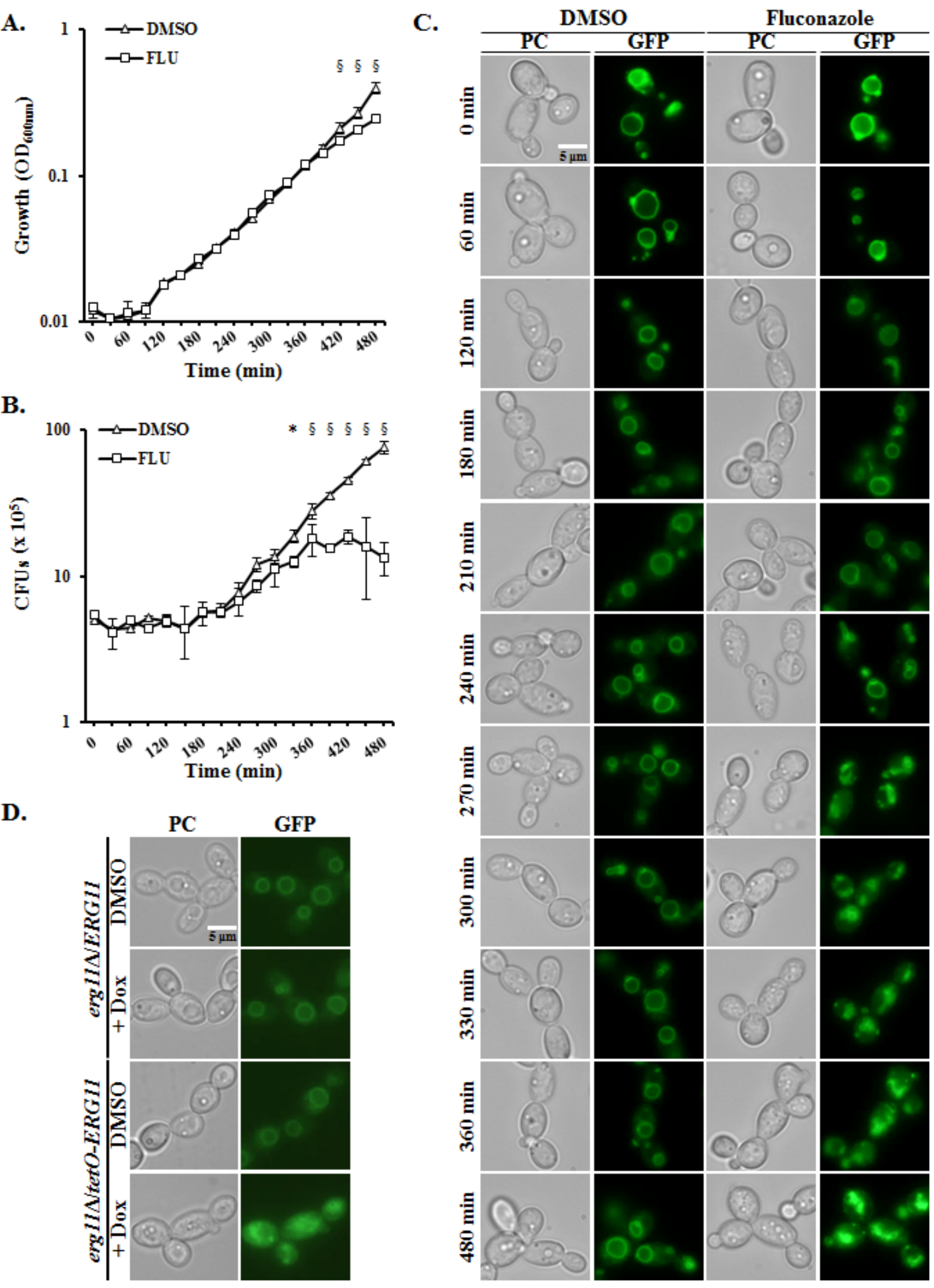
Figure 2-4. Azole treatment severely disrupts the integrity of $C$. albicans vacuole. A fusion protein consisting of the first 129 amino acids of Carboxypeptidase Y (Cpylp) fused to GFP was expressed in C. albicans under the ACT1 promoter, in order to fluorescently label the vacuolar lumen. YNB broth $\pm 1 \mu \mathrm{g} / \mathrm{mL}$ of fluconazole (FLU) was inoculated at $1 \times 10^{6}$ cells $/ \mathrm{mL}$. Samples were taken hourly and vacuolar integrity was observed by fluorescence microscopy using a FITC filter set. 


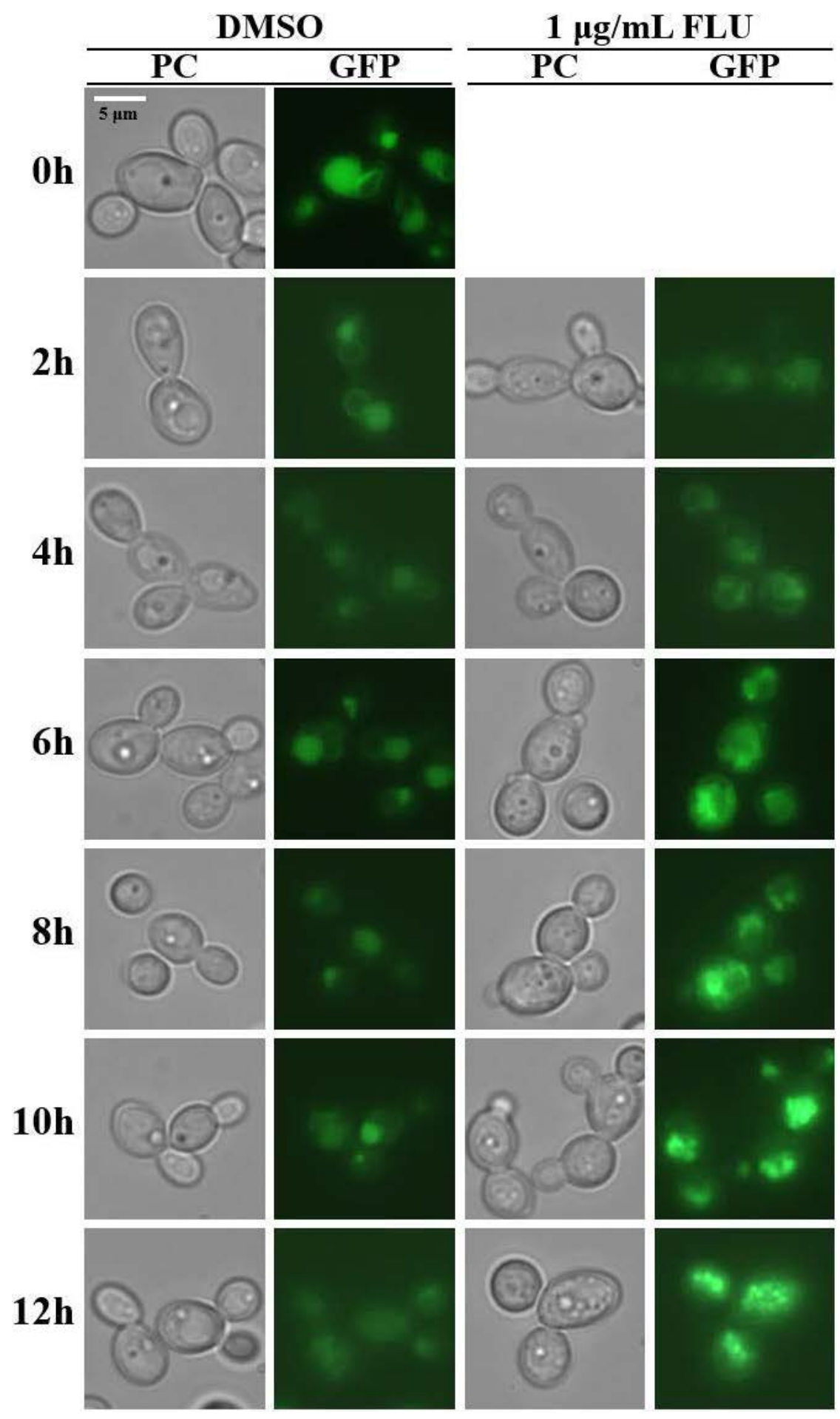


induced by azole treatment are a direct consequence of Erg $11 \mathrm{p}$ inhibition, and thus presumably result from depletion of cellular ergosterol.

\section{Erg3p modulates the severity of vacuole disruption in azole treated $C$. albicans}

Ergosterol depletion together with accumulation of the toxic sterol $14 \alpha-$ methylergosta-8,24(28)-dien-3 $\beta, 6 \alpha$-diol in the plasma membrane following azole treatment are thought to result in loss of its integrity and ultimately growth arrest (141). The production of $14 \alpha$-methylergosta-8,24(28)-dien-3 $\beta, 6 \alpha$-diol depends upon Erg3p (C-5 sterol desaturase) activity since loss of Erg3 $p$ function results in the accumulation of its precursor $14 \alpha$-methylfecosterol in the plasma membrane, which allows continued growth following azole treatment $(81,141)$. Whether production of toxic sterols mediated by Erg3p upon azole exposure contributes to loss of integrity of intracellular membranes such as the fungal vacuole is unknown.

In order to test whether Erg3p function impacts vacuole integrity after azole exposure, we constructed an $\operatorname{erg} 3 \Delta / \Delta$ mutant expressing the fusion protein GFP-Ypt72p and determined its vacuole morphology by fluorescence microscopy. As shown in Figure 2-5, the $\operatorname{erg} 3 \Delta / \Delta$ mutant exhibited a rather normal vacuole morphology in the absence of fluconazole. While treatment with $2 \mu \mathrm{g} / \mathrm{mL}$ of fluconazole caused severe loss of vacuole integrity in the WT control strain as previously shown, the severity of vacuole disruption in the $\operatorname{erg} 3 \Delta / \Delta$ mutant was reduced under similar conditions (Figure 2-5). This suggests that an active Erg3p, and thus presumably the toxic sterols that it synthesizes following azole treatment, exacerbates the severity of vacuole disruption.

\section{Discussion}

Azoles inhibition of fungal growth is often assumed to be a consequence of plasma membrane dysfunction caused by both depletion of cellular ergosterol, and the accumulation of 'toxic' sterol intermediates (196). Here, we established that inhibition of lanosterol demethylase (Erg11p) with azoles or through transcriptional repression led to significant perturbation of vacuolar integrity in the fungal pathogen C. albicans. Moreover, vacuolar degeneration was an early consequence of azole treatment. We have previously demonstrated that $C$. albicans mutants with severe defects in vacuolar biogenesis, have impaired growth, stress tolerance, polarized hyphal growth and pathogenicity $(49-51,166)$. Thus it is possible that vacuolar dysfunction may make a significant contribution to the overall antifungal activity of the azoles. Previous studies have also noted that several non-essential Saccharomyces cerevisiae ergosterol biosynthetic mutants have a fragmented vacuole morphology, and ergosterol is required for homotypic vacuole-vacuole fusion $(170,171)$. We have shown that mutants of $C$. albicans lacking Erg2p or Erg24p, the targets of the morpholine antifungals, have distinct degrees of degeneration of vacuolar integrity (172), which is worsened when both targets are pharmacologically repressed with ergosterol biosynthesis inhibitors such as amorolfine and fenpropimorph. Like the C. albicans erg $2 \Delta / \Delta$ and $\operatorname{erg} 24 \Delta / \Delta$ mutants, the 


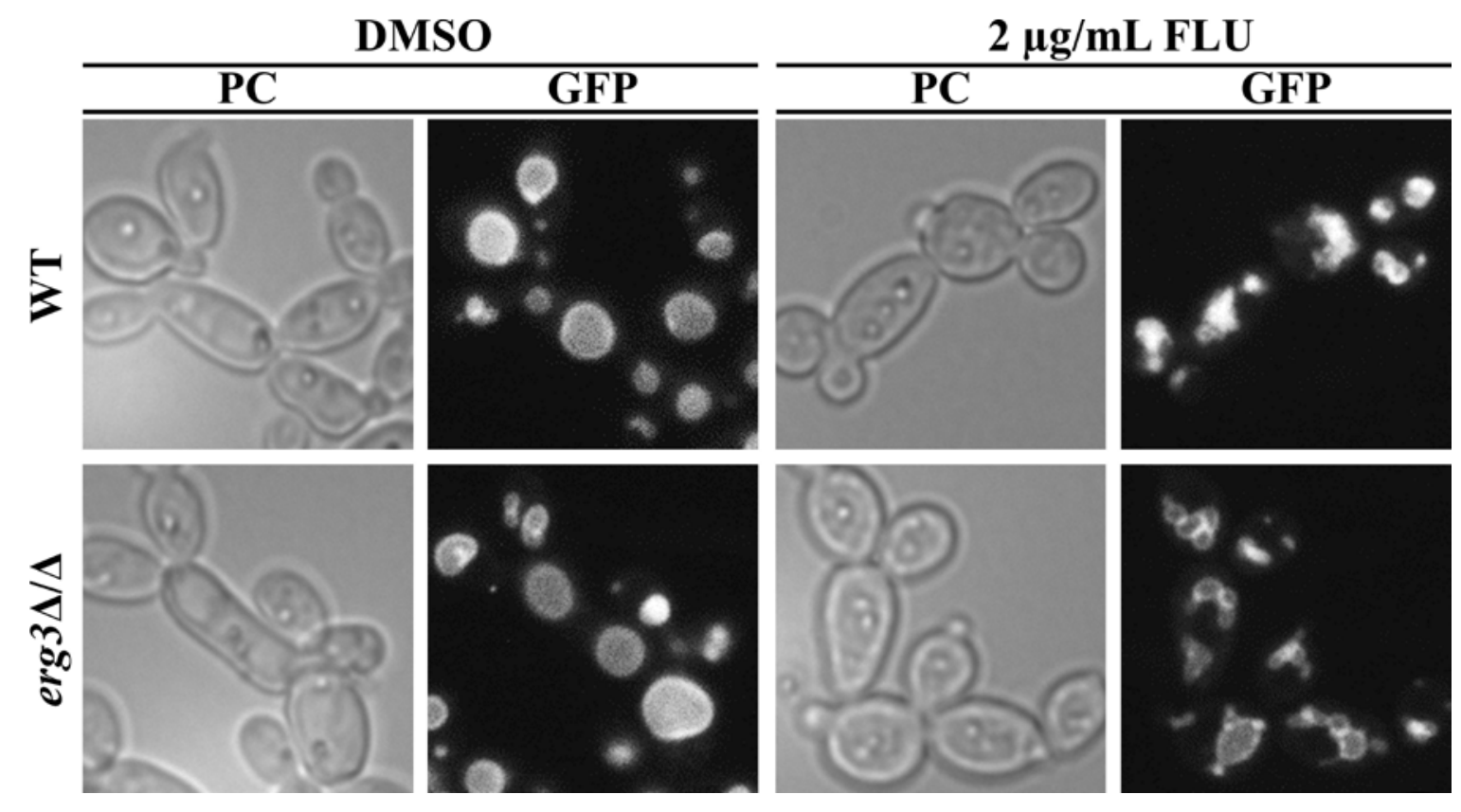

Figure 2-5. Loss of Erg3p reduces the impact of azole treatment upon vacuolar integrity in $C$. albicans.

C. albicans WT (CAI4) and erg $3 \Delta / \Delta$ mutant strains expressing the GFP-Ypt72p fusion protein were treated with $2 \mu \mathrm{g} / \mathrm{mL}$ of fluconazole or $0.5 \%$ DMSO for 6 hours in YNB medium. Vacuole morphology was determined with a Cytation ${ }^{\mathrm{TM}} 5$ Cell Imaging MultiMode Reader using a GFP filter cube. PC: phase contrast. 
$\operatorname{erg} 3 \Delta / \Delta$ mutant is expected to have no ergosterol. However, the $\operatorname{erg} 3 \Delta / \Delta$ mutant has a lesser degree of vacuolar degeneration than the former mutants. In addition, the $C$. albicans $\operatorname{erg} 3 \Delta / \Delta$ mutant has a lesser degree of deterioration of vacuolar integrity when treated with azole antifungals than a WT strain. This indicates that the degree of vacuolar degeneration does not depend only on the absence of ergosterol, but rather depends on the specific steps blocked along the ergosterol biosynthetic pathway. This may be explained by differences in the physical or chemical properties of the specific sterol intermediates that accumulate following blockage of a particular step. Different sterol species have been shown biochemically to confer different structural properties upon membranes (197), and thus the accumulation of a particular sterol may alter the structure of a cellular membrane.

Recent studies by Rao and colleagues have also suggested that ergosterol is required for the activity of the V-ATPase responsible for vacuolar acidification, which in turn is required for normal vacuolar membrane fusion $(198,199)$. However, it is likely that ergosterol depletion in itself affects the structural integrity of the vacuole, and potentially the activity of a multitude of other vacuolar membrane proteins. Thus the precise interdependence between ergosterol availability, V-ATPase activity, vacuolar integrity and the antifungal efficacy of the azoles, remains to be elucidated. 


\section{CHAPTER 3. VACUOLAR TRAFFICKING THROUGH THE LATE ENDOSOME/PREVACUOLAR COMPARTMENT SIGNIFICANTLY IMPACTS AZOLE SUSCEPTIBILITY OF CANDIDA ALBICANS*}

\section{Introduction}

Recent evidence points to a significant role of ergosterol in the function of the fungal vacuole. For example, ergosterol is required for endocytosis (169) and homotypic vacuole fusion $(170,171)$, and has been suggested to be required for the activity of the VATPase responsible for vacuolar acidification (79). In Chapter 2 we showed that Erg11p inhibition following exposure to azole antifungals drastically impacts the integrity of the C. albicans vacuole and this is dependent upon Erg11p inhibition. Moreover, we determined that severe fragmentation of the vacuole can be observed as an early event following azole treatment, occurring before significant growth inhibition is observed. Thus it is likely that the vacuolar defects observed upon azole treatment contribute to the antifungal activity of the azoles, as vacuolar dysfunction renders $C$. albicans more susceptible to a wide variety of stresses, can impair growth and hyphae development, and reduces its pathogenic capacity in vivo $(49,51,166)$. Nevertheless, whether vacuolar dysfunction can impact the antifungal activity of the azoles is unknown.

Normal membrane trafficking to the fungal vacuole is required for $C$. albicans to respond to a multitude of stressors, hyphal growth, biofilm formation and its ability to cause disease. Many mutants with impaired vacuolar membrane trafficking are deficient in one or more of the aforesaid biological processes $(50,51,187,200)$. Several membrane trafficking pathways that deliver material to the vacuole have been characterized in the non-pathogenic model yeast $S$. cerevisiae (Figure 3-1), all of which make significant contributions to normal vacuolar function (201). The alkaline phosphatase (ALP) trafficking pathway delivers its cargo directly from the Golgi to the vacuole (Figure 3-1). Membrane trafficking through the ALP pathway requires the AP3 adaptor complex which consists of 4 proteins namely Apl6p, Apl5p, Apm3p and Aps3p (201).

The so called carboxypeptidase Y (CPY) trafficking pathway transports cargo from the Golgi to the vacuole, via an intermediate trafficking step at the late endosome/pre-vacuolar compartment (PVC, Figure 3-1). Targeting and fusion of Golgiderived vesicles with the PVC require the t-SNARE [Soluble N-ethylmaleimide-sensitive factor (NSF) attachment protein receptor] Pep12p/Vps6p in the target membrane (202), the v-SNARE protein Vtilp on the transport vesicle (203), the SM (Sec1/Munc18p-like)

\footnotetext{
* Modified by permission of American Society for Microbiology. Luna-Tapia A, Kerns ME, Eberle KE, Jursic BS, Palmer GE. 2015. Trafficking through the late endosome significantly impacts Candida albicans tolerance of the azole antifungals. Antimicrob Agents Chemother 59:2410-2420. doi:10.1128/AAC.04239-14.
} 


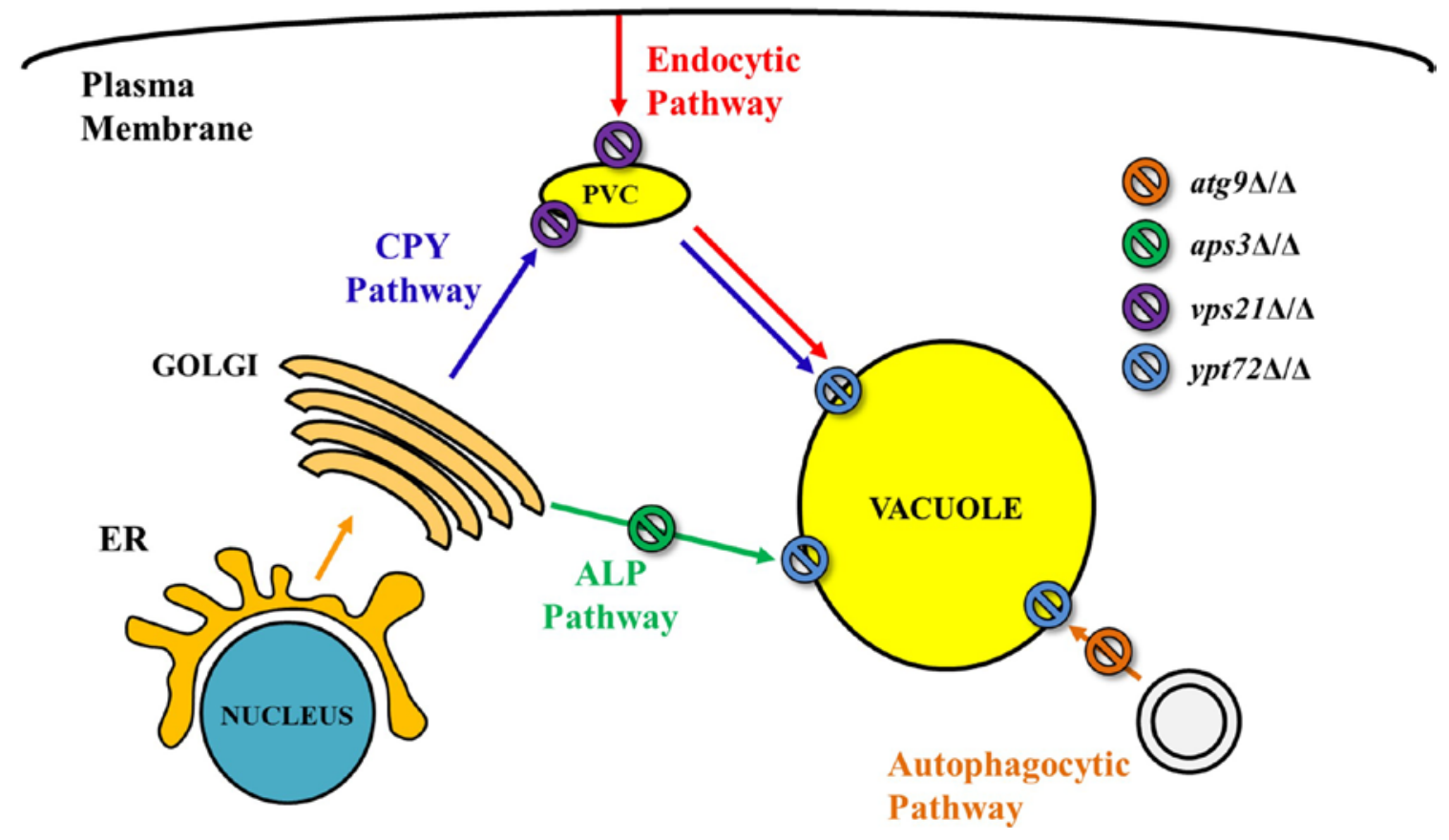

Figure 3-1. Membrane trafficking pathways to the vacuole of $C$. albicans analyzed in this study.

The alkaline phosphatase pathway (ALP) delivers material from the Golgi apparatus to the vacuole and is dependent upon Aps3p. Both the carboxypeptidase Y (CPY) and the endocytic pathways carry material to the vacuole from the Golgi apparatus or the plasma membrane, respectively, via the late endosome/prevacuolar compartment; both are dependent upon Vps21p. The autophagocytic pathway transports material from the cytoplasm to the vacuole and is dependent upon Atg9p. Additionally, the role of Ypt72p (the C. albicans homolog of yeast's Ypt7p) which controls membrane fusion events at the vacuole itself, was analyzed in this study. Mutants blocked in ALP trafficking (aps3 $\Delta / \Delta)$, endosomal trafficking $(v p s 21 \Delta / \Delta)$, autophagy $(\operatorname{atg} 9 \Delta / \Delta)$ or membrane fusion at the vacuolar membrane $(y p t 72 \Delta / \Delta)$ were examined. 
protein Vps41p, the small Rab GTPase Vps21p (204), the Rab exchange factor Vps9p (205), the Rab effector Vac1p/Vps19p/Pep7p (206), and membrane tethering factors of the class C Vps complex formed by Vps11p/Pep5p, Vps18p/Pep3p, Vps16p, and Vps33p (201). Once at the PVC, proteins destined for the vacuole are sorted, packed into membrane vesicles for transport to and fusion with the vacuolar membrane, or for retrograde transport back to the Golgi (201).

The endocytic trafficking pathway carries out the internalization of extracellular material and plasma membrane components for recycling or degradation in the fungal vacuole via endocytosis. Similar to the CPY pathway, the endocytic pathway converges at the PVC (201). At this stage, the fusion of vesicles arriving from the plasma membrane with the $\mathrm{PVC}$ requires the same components regulating membrane fusion of vesicles transported through the CPY trafficking pathway, including the Rab GTPase Vps21p (207).

During autophagy, bulk or selected material from the cytoplasm is sequestered into double-membrane compartments called autophagosomes that are delivered to the vacuole for degradation (Figure 3-1). Autophagy is induced in response to multiple stresses including nutrient starvation (208), or certain developmental events (209). Among the various proteins involved in autophagocytic trafficking, Atg9p is strictly required for autophagosome formation (210).

The last trafficking step of the ALP, CPY and autophagocytic pathways is characterized by fusion of transport vesicles with the vacuole (Figure 3-1), a process controlled by the Rab GTPase Ypt7p in yeast, together with other components that include the SNARE complex formed by Vam3p, Vtilp, Vps43p/Vam7p and Ykt6p, and the Class C complex $(203,211,212)$.

The objective of the studies described in this chapter was to determine if vacuolar dysfunction, in the context of membrane trafficking to the vacuole, can impact azole susceptibility in C. albicans.

\section{Materials and Methods}

\section{Growth and media conditions}

C. albicans was routinely grown on yeast extract-peptone-dextrose (YPD) at $30^{\circ} \mathrm{C}$, supplemented with $50 \mu \mathrm{g} / \mathrm{mL}$ uridine when necessary. Transformant selection was carried out on minimal YNB medium $(6.75 \mathrm{~g} / \mathrm{L}$ yeast nitrogen base without amino acids, $2 \%$ dextrose, $2 \%$ Bacto agar), supplemented with the appropriate auxotrophic requirements as described for $S$. cerevisiae (185) or $50 \mu \mathrm{g} / \mathrm{mL}$ uridine. 


\section{Plasmid construction}

Plasmid pLUX (213), was kindly provided by Dr. William Fonzi (Georgetown University). Plasmids pLUXVPS21 and pKE1 have been previously described $(51,187)$. Plasmid pGUD1 was constructed as described in Chapter 2. All oligonucleotides used in this study are listed in Table 3-1.

\section{C. albicans strain construction}

Strains used in this study are listed in Table 3-2. The vps $21 \Delta / \Delta, y p t 72 \Delta / \Delta$, $\operatorname{atg} 9 \Delta / \Delta, \operatorname{aps} 3 \Delta / \Delta, y p t 52 \Delta / \Delta, y p t 53 \Delta / \Delta, v p s 21 \Delta / \Delta y p t 52 \Delta / \Delta, v p s 21 \Delta / \Delta y p t 53 \Delta / \Delta$, $y p t 52 \Delta / \Delta y p t 53 \Delta / \Delta$ and $v p s 21 \Delta / \Delta y p t 52 \Delta / \Delta y p t 53 \Delta / Y P T 53^{T 27 N}$ mutants were constructed in previous studies $(50,51,187,214)$. The $C$. albicans pep $12 \Delta / \Delta$ mutant and isogenic control strains (200), were kindly provided by Dr. Samuel A. Lee (University of New Mexico). The azole susceptible clinical isolate TW1 [isolate 1 from reference (125)] and the matched azole resistant isolate TW17 [isolate 17 from reference (125)] were kindly provided by Dr. Theodore C. White (University of Missouri-Kansas City). Control strain YJB6284 (215) was kindly provided by Dr. Judith Berman (University of Minnesota) and SC5314 has been previously described (216).

C. albicans was transformed with DNA constructs using the lithium acetate procedure (217). Gene deletion strains were constructed by the PCR-based approach described by Wilson et al., (190), using the $u \mathrm{ra} 3 \Delta / \Delta$ his $1 \Delta / \Delta \arg 4 \Delta / \Delta$ strain BWP17 (kindly provided by Dr. Aaron Mitchell, Carnegie Mellon University). The doxycycline repressible tetO-ERG11 strains were made using the system described by Nakayama and colleagues (191) as described in Chapter 2.

\section{Antifungal susceptibility testing}

Antifungal susceptibility testing of all the strains included in this study was performed using the broth micro-dilution method described in the CLSI document M27A3 (218) in a 96-well plate format. All drugs for susceptibility testing used in this study were diluted in DMSO in 2-fold dilutions at 200 times the final concentration. Fluconazole (Sigma-Aldrich) concentrations tested ranged from $64 \mu \mathrm{g} / \mathrm{mL}$ to 0.0313 $\mu \mathrm{g} / \mathrm{mL}$, Miconazole (Sigma-Aldrich) from $50 \mu \mathrm{g} / \mathrm{mL}$ to $0.024 \mu \mathrm{g} / \mathrm{mL}$, Ketoconazole (Sigma-Aldrich) from $32 \mu \mathrm{g} / \mathrm{mL}$ to $0.015 \mu \mathrm{g} / \mathrm{mL}$, Itraconazole (Sigma-Aldrich) from 16 $\mu \mathrm{g} / \mathrm{mL}$ to $0.007 \mu \mathrm{g} / \mathrm{mL}$ and Amphotericin B (LKT Laboratories, Inc.) from $16 \mu \mathrm{g} / \mathrm{mL}$ to $0.001 \mu \mathrm{g} / \mathrm{mL}$. RPMI 1640 medium (Sigma-Aldrich) was prepared according to the CLSI document. The medium was buffered with $0.165 \mathrm{M}$ morpholinepropanesulfonic acid (MOPS) and $\mathrm{pH}$ adjusted using $\mathrm{NaOH}$ and $\mathrm{HCl}$. Cell inoculum was $\sim 1 \times 10^{3}$ cells per well. Plates were incubated without shaking at $35^{\circ} \mathrm{C}$ for 24 or 48 hours unless otherwise stated. The content of each well was carefully resuspended by pipetting up and down before $\mathrm{OD}_{600 \mathrm{~nm}}$ was measured using a Biotek Synergy Mx plate reader. 
Table 3-1. List of oligonucleotides used in the study described in Chapter 3.

\begin{tabular}{ll}
\hline \multicolumn{1}{c}{ Primer } & \multicolumn{1}{c}{ Sequence (5'-3') } \\
\hline ERG11AMPF2-KpnI & TCAGGTACCAATGAAAATGTCGTGGGGAGC \\
& TTCTTCCATATTACTTGTCTTCTTTATTATATATATAAG \\
ERG11DISF & TTTCTTTCAAGAAGATCATAACTCAATGTTTTCCCAGTC \\
& ACGACGTT \\
ERG11DISR & TGTGTTAATCCAACTAAGTAACAAAATGAAAACAATCTG \\
& AACACTGAATCGAAAGAAAGTTGCCGTTTTATGTGGAAT \\
ERG11R-KpnI-MIuI & TGTGAGCGGATA \\
ERG11SEQR1 & TTTTCATAACATTGGCAACCC \\
& AAATCAATTTTATATATAAATAGACAAAGAAAGGGAAT \\
ERG11TETOF & TCAATCGTTATTCTTCCATATTACTTGTCGTAATACGACT \\
& CACTATAGGG \\
ERG11TETOR & ATCTGTTGTGTAACACTAAGGGACAAAAAATAATTAATG \\
& CCATCAATGACAGTTTCAACAATAGCCATCTAGTTTTCT \\
LUXINTDETF & GAGATAAAGCTG \\
LUXINTDETR & CTGACCTTTAGTCTTTCCTGC \\
TETODETF & CAGTAGTACTTGTTGTTGTATCG \\
URA3DISF1 & GTTGACACTTCTAAATAAGCG \\
URA3DISR2 & ATGACAGTCAACACTAAGACC \\
URA3INTF2 & CAAATAAGCATTCCAACCAGC \\
URA3INTR & TTATACCATCCAAATCCCGCG \\
VPS21AMPR & CAATTATAAATGTGAAGGGGG \\
VPS21DETF & TCATCAGGATCCGTAAAATTGCACGACTGTGCC \\
VPS21DETF & GAGATATAACGATTGCATTGG \\
& GCAACATGTGCTTCTTGGAGC \\
VPS21DISF & CCATCATTATTTCTACTATTTGTATTGCATTGTCGCATT \\
& CAGGATCAAAGAGCCAAAATACATACTAGCATGTGGA \\
VPS21DISR & ATTGTGAGCGGATA \\
VPS21SEQF & ACCATGGAGATCTGTACACACTTGCCTATTTGTATATTA \\
\hline & CCTTAACTTCAGTCTCCTTGTTATTTATATTTTCCCAGT \\
& CACGACGTT \\
\hline
\end{tabular}


Table 3-2. List of strains used in the study described in Chapter 3.

\begin{tabular}{|c|c|c|}
\hline Strain & Relevant Genotype & Reference \\
\hline SC5314 & Wild-type/clinical isolate & \\
\hline BWP17 & $\operatorname{ura} 3 \Delta / \Delta$ his $1 \Delta / \Delta \arg 4 \Delta / \Delta$ & $(190)$ \\
\hline YJB6284 & 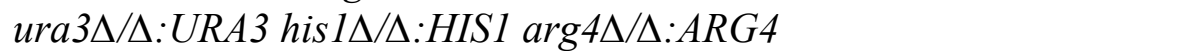 & $(215)$ \\
\hline THE1 & ura $3 \Delta / \Delta$ ade $2 \Delta / \triangle E N O 1 / \Delta: E N O 1$-tetR-ScHAP4AD-3 $\times$ HA-ADE2 & $(191)$ \\
\hline TW1 & Matched azole susceptible clinical isolate & $(125)$ \\
\hline TW17 & Matched azole resistant clinical isolate & $(125)$ \\
\hline$v p s 21 \Delta / \Delta$ & ura3 $\Delta / \Delta: U R A 3$ his $1 \Delta / \Delta \arg 4 \Delta / \Delta$ vps $21 \Delta: H I S 1 / v p s 21 \Delta: A R G 4$ & $(51)$ \\
\hline vps $21 \Delta / \Delta+V P S 21$ & ura3 $\Delta / \Delta: U R A 3: V P S 21$ his $1 \Delta / \Delta \arg 4 \Delta / \Delta$ vps $21 \Delta: H I S 1 / v p s 21 \Delta: A R G 4$ & $(51)$ \\
\hline 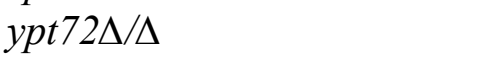 & 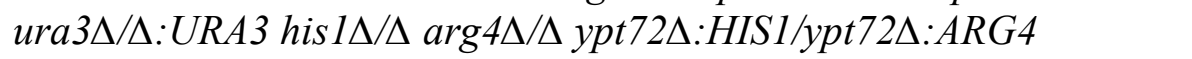 & $(51)$ \\
\hline 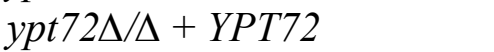 & 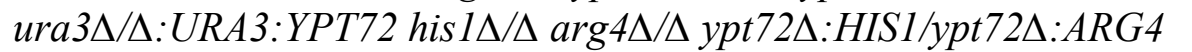 & $(51)$ \\
\hline $\operatorname{atg} 9 \Delta / \Delta$ & ura3 $\Delta / \Delta: U R A 3$ his $1 \Delta / \Delta \arg 4 \Delta / \Delta \operatorname{atg} 9 \Delta: H I S 1 / \operatorname{atg} 9 \Delta: A R G 4$ & $(214)$ \\
\hline $\operatorname{atg} 9 \Delta / \Delta+A T G 9$ & 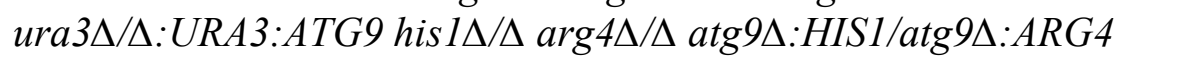 & $(214)$ \\
\hline $\operatorname{aps} 3 \Delta / \Delta$ & ura $3 \Delta / \Delta: U R A 3$ his $1 \Delta / \Delta \arg 4 \Delta / \Delta$ aps $3 \Delta: H I S 1 / a p s 3 \Delta: A R G 4$ & $(50)$ \\
\hline $\operatorname{aps} 3 \Delta / \Delta+A P S 3$ & ura $3 \Delta / \Delta: U R A 3: A P S 3$ his $1 \Delta / \Delta \arg 4 \Delta / \Delta$ aps $3 \Delta: H I S 1 / a p s 3 \Delta: A R G 4$ & $(50)$ \\
\hline 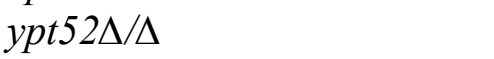 & 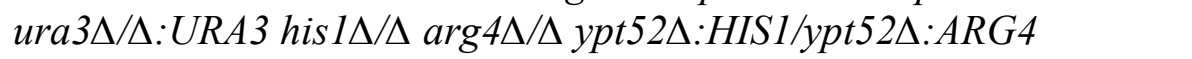 & $(187)$ \\
\hline$y p t 52 \Delta / \Delta+Y P T 52$ & 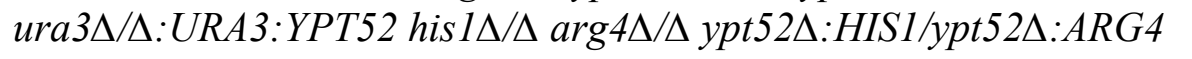 & $(187)$ \\
\hline ypt $53 \Delta / \Delta$ & 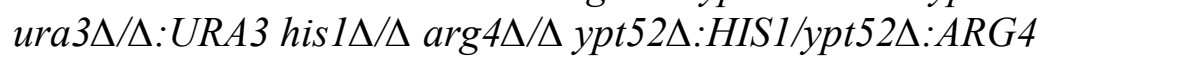 & $(187)$ \\
\hline 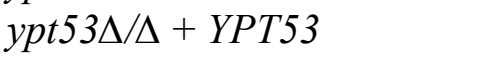 & 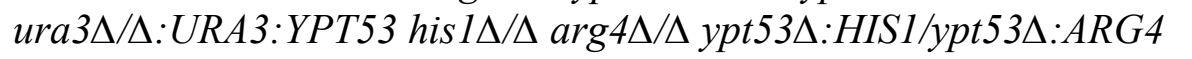 & $(187)$ \\
\hline$y p t 52 \Delta / \Delta y p t 53 \Delta / \Delta$ & 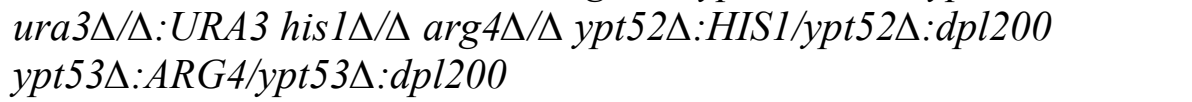 & $(187)$ \\
\hline$y p t 52 \Delta / \Delta y p t 53 \Delta / \Delta+Y P T 52$ & 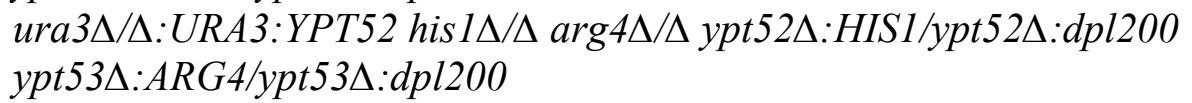 & $(187)$ \\
\hline$y p t 52 \Delta / \Delta y p t 53 \Delta / \Delta+Y P T 53$ & 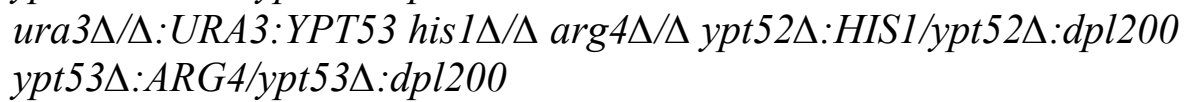 & $(187)$ \\
\hline$v p s 21 \Delta / \Delta y p t 52 \Delta / \Delta$ & 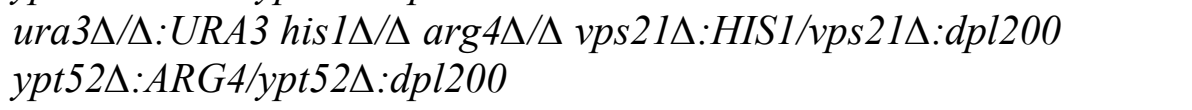 & $(187)$ \\
\hline
\end{tabular}




\section{Table 3-2. (Continued).}

\begin{tabular}{|c|c|c|}
\hline Strain & Relevant Genotype & Reference \\
\hline$v p s 21 \Delta / \Delta y p t 52 \Delta / \Delta+V P S 21$ & 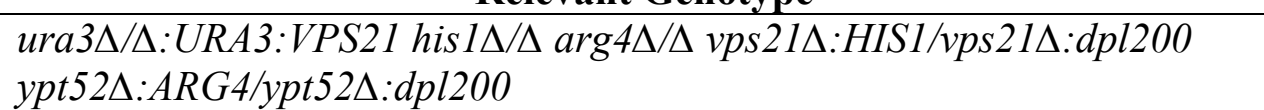 & $(187)$ \\
\hline vps $21 \Delta / \Delta y p t 52 \Delta / \Delta+Y P T 52$ & 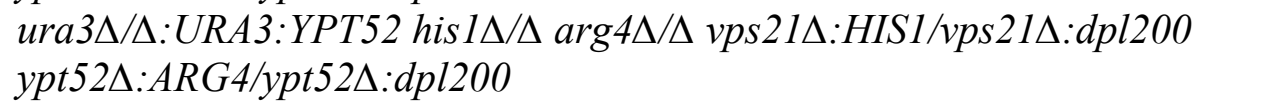 & $(187)$ \\
\hline $\operatorname{vps} 21 \Delta / \Delta y p t 53 \Delta / \Delta$ & 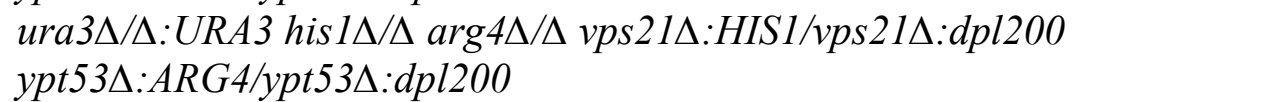 & $(187)$ \\
\hline$v p s 21 \Delta / \Delta y p t 53 \Delta / \Delta+V P S 21$ & 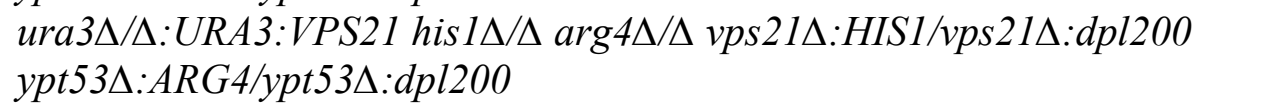 & $(187)$ \\
\hline vps $21 \Delta / \Delta y p t 53 \Delta / \Delta+Y P T 53$ & 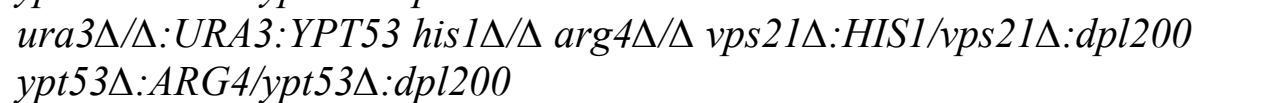 & $(187)$ \\
\hline vps $21 \Delta / \Delta y p t 52 \Delta / \Delta y p t 53 \Delta / Y P T 53^{T 27 N}$ & 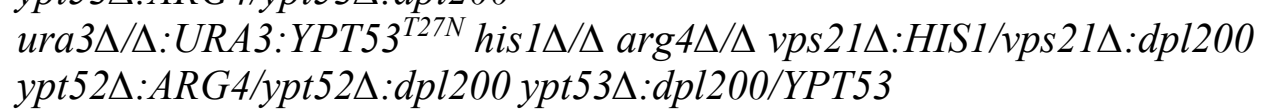 & $(187)$ \\
\hline pep $12 \Delta / \Delta$ & ura $3 \Delta / \Delta \arg 4 \Delta / \Delta$ his $1 \Delta / \Delta: H I S 1$ pep $12 \Delta:$ dpl200/pep12A:ARG4 & $(200)$ \\
\hline pep $12 \Delta / \Delta+$ PEP12 & ura $3 \Delta / \Delta \arg 4 \Delta / \Delta$ his $1 \Delta / \Delta: H I S 1: P E P 12$ pep $12 \Delta: d p l 200 / p e p 12 \Delta: A R G 4$ & $(200)$ \\
\hline $\operatorname{erg} 11 \Delta / E R G 11$ & 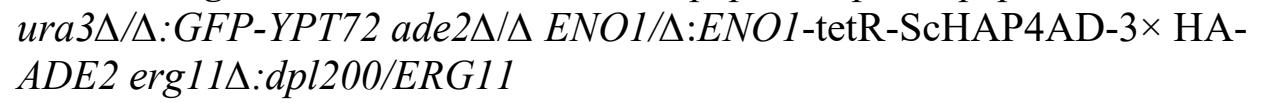 & This study \\
\hline $\operatorname{erg} 11 \Delta / \operatorname{tetO}-E R G 11$ & $\begin{array}{l}\text { ura3 } \Delta / \Delta: U R A 3 \text { ade } 2 \Delta / \triangle E N O 1 / \Delta: E N O 1: \text { tetR-ScHAP4AD-3× HA-ADE2 } \\
\text { erg11 } 1 \Delta: \text { dpl200/tet } O-E R G 11\end{array}$ & This study \\
\hline $\operatorname{erg} 11 \Delta / \operatorname{tetO}-E R G 11$ vps $21 \Delta / \Delta$ & $\begin{array}{l}\text { ura3 } \Delta / \Delta: U R A 3 \text { ade } 2 \Delta / \triangle E N O 1 / \Delta: E N O 1: \text { tetR-ScHAP4AD-3× HA-ADE2 } \\
\text { erg11 }: \text { dpl200/tetO-ERG11 vps } 21 \Delta: d p l 200 / v p s 21 \Delta: d p l 200\end{array}$ & This study \\
\hline $\operatorname{erg} 11 \Delta / \operatorname{tetO}-E R G 11$ vps $21 \Delta / \Delta+V P S 21$ & 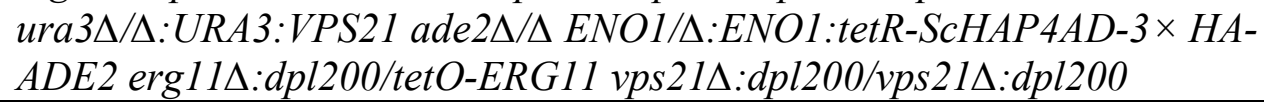 & This study \\
\hline
\end{tabular}




\section{Quantification of cellular ergosterol}

$50 \mathrm{~mL}$ YNB broth plus either $4 \mu \mathrm{g} / \mathrm{mL}$ fluconazole or $0.5 \%$ DMSO was inoculated with $2 \times 10^{6} \mathrm{C}$. albicans cells $/ \mathrm{mL}$ and grown at $35^{\circ} \mathrm{C}$ for 24 hours with shaking at $200 \mathrm{rpm}$. Total cellular sterols were then extracted using n-heptane as described by Arthington-Skaggs et al. (219). n-heptane from the organic phase was evaporated under reduced pressure $(0.03 \mathrm{~mm} \mathrm{Hg})$ at $-20^{\circ} \mathrm{C}$. White powdery residue was dissolved in $0.5 \mathrm{~mL} \mathrm{CDCl}_{3}(99.8 \% \mathrm{D}$ contains $1 \% \mathrm{v} / \mathrm{v} \mathrm{TMS})$ and it was subject to proton NMR (Nuclear magnetic resonance) spectroscopy analysis. NMR was recorded on Varian $500 \mathrm{MHz}$ INOVA instrument. The amounts of the ergosterol in these samples were estimated by comparison with the NMR integrals of methyl C-18 group at 0.562 ppm to an internal TMS standard. The amount of ergosterol in these samples was calculated by standardizing these integrals with the C-18 methyl integral of a standard ergosterol sample (1 mg/mL; Sigma-Aldrich) that was prepared and the NMR was recorded by an identical procedure.

\section{Results}

\section{Vps21p mediated trafficking through the pre-vacuolar compartment influences fungal growth in the presence of azoles}

In the previous chapter we revealed that treatment with azole antifungals cause severe disruption of $C$. albicans vacuolar integrity. To gain further insight into the interaction between the antifungal activity of the azoles and vacuolar integrity, we tested the susceptibility of $C$. albicans mutants blocked in four distinct vacuolar trafficking steps (Figure 3-1), using the CLSI (Clinical and Laboratory Standards Institute) standard protocol for antifungal susceptibility testing (218). The susceptibility of mutants blocked in either autophagy $(\operatorname{atg} 9 \Delta / \Delta)(214)$, or in a direct Golgi-to-vacuole trafficking pathway $(\operatorname{aps} 3 \Delta / \Delta)(50)$, to miconazole (Figure 3-2A and 2B) and fluconazole (Figure 3-3A and 3B) is not significantly different from their isogenic control strains. However, a $v p s 21 \Delta / \Delta$ mutant, lacking a Rab GTPase that regulates membrane fusion and trafficking through the pre-vacuolar compartment (PVC), apparently has significantly reduced susceptibility to miconazole (Figure 3-2C), fluconazole (Figure 3-3C), itraconazole (Figure 3-4A) and ketoconazole (Figure 3-4B) as judged by the enhanced growth of the mutant versus the control strains in the presence of any of these azole antifungals. Interestingly, a $y p t 72 \Delta / \Delta$ mutant lacking a Rab GTPase (the homolog of Ypt7p of S. cerevisiae) that localizes to the vacuole itself also showed slightly elevated growth in the presence of both fluconazole and miconazole (Figure 3-3D and Figure 3-2D, respectively), but the difference was less dramatic than for the vps $21 \Delta / \Delta$ mutant. This suggests that trafficking through the PVC is more important with respect to determining azole sensitivity, than trafficking to the vacuole per se. The susceptibility of the vps $21 \Delta / \Delta$ mutant to Amphotericin $\mathrm{B}$, which acts by directly binding ergosterol, is not significantly different from the isogenic control strain (Figure 3-4C). Furthermore, the vps $21 \Delta / \Delta$ mutant has an increased susceptibility to the allylamine antifungal terbinafine (Figure 3-5A), which 
A.
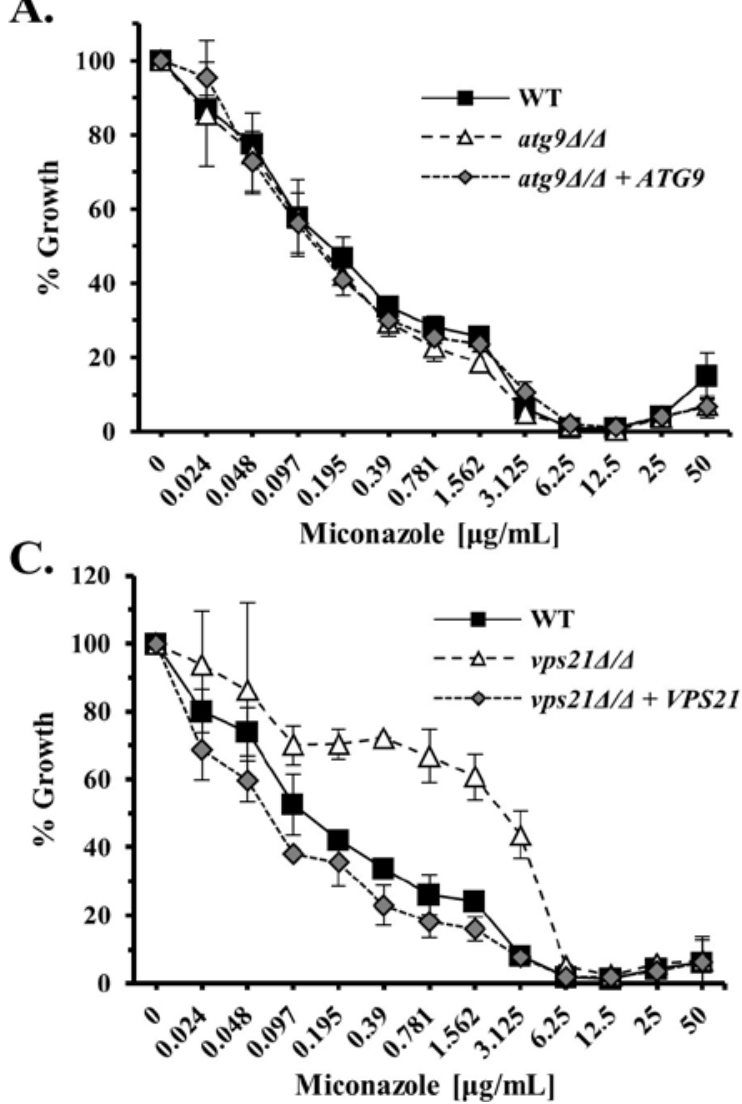

B.

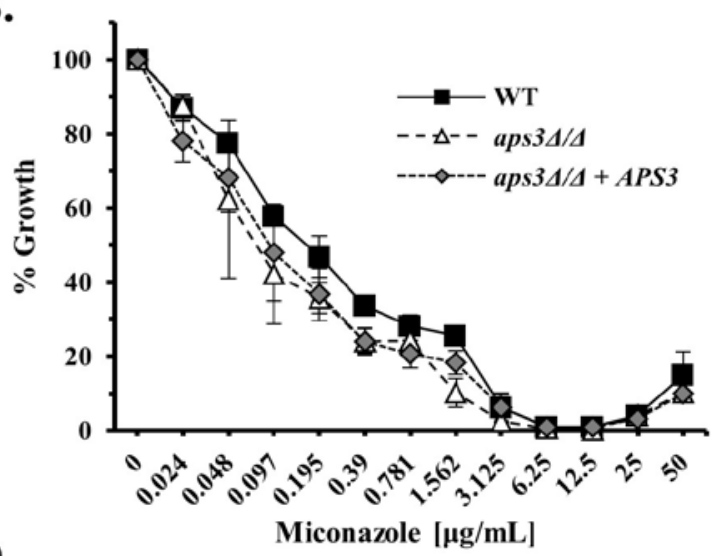

D.

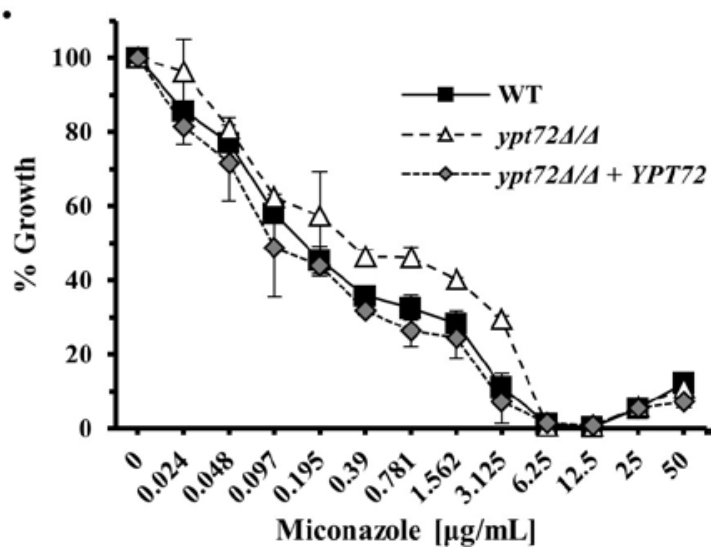

Figure 3-2. Endosomal trafficking influences the growth of $C$. albicans in the presence of miconazole.

The susceptibility of several C. albicans vacuolar trafficking mutants to miconazole was compared to 'wild-type' (YJB6284) and 'reconstituted' control strains using the standard CLSI broth microdilution protocol. After 48 hours incubation, growth was measured as $\mathrm{OD}_{600 \mathrm{~nm}}$ and expressed as a percentage of the growth in the minus drug (DMSO alone) control wells. (A) An atg $9 \Delta / \Delta$ mutant blocked in autophagy. (B) An aps $3 \Delta / \Delta$ mutant blocked in a direct Golgi-to-vacuole trafficking pathway. (C) A vps21 $1 \Delta / \Delta$ mutant blocked in membrane fusion and trafficking through the PVC. (D) An $y p t 72 \Delta / \Delta$ mutant blocked in membrane fusion at the vacuolar membrane. The mean and standard deviation of two independent experiments are presented in each panel. 
A.

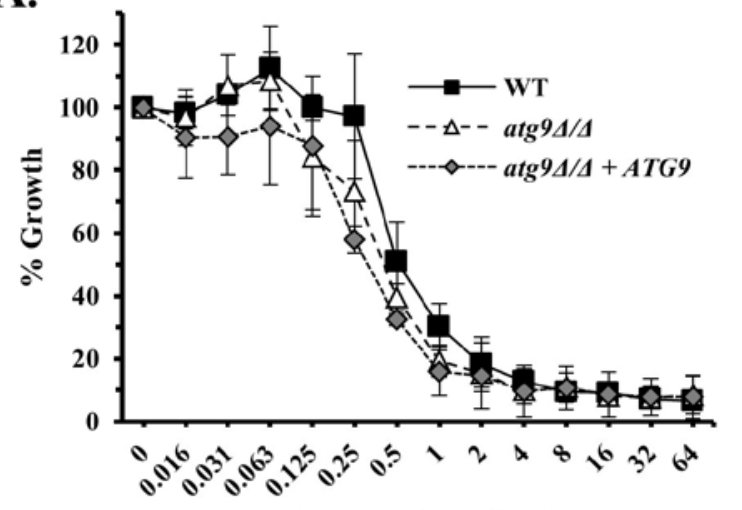

C.

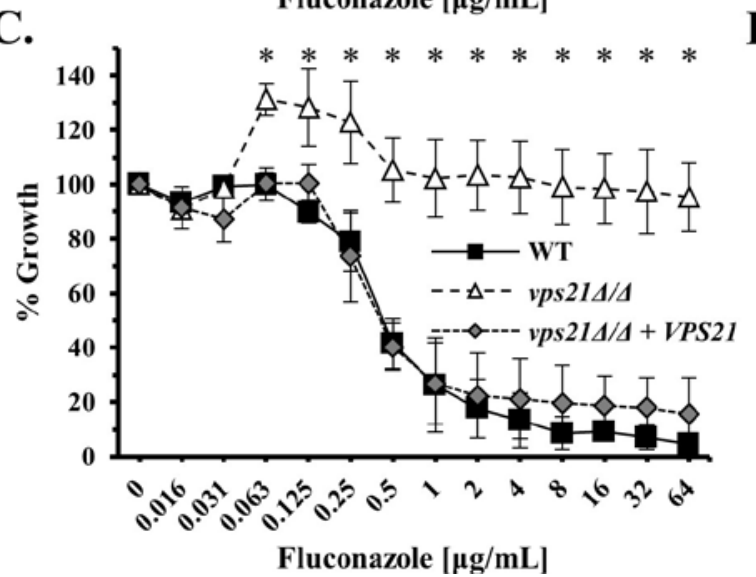

B.

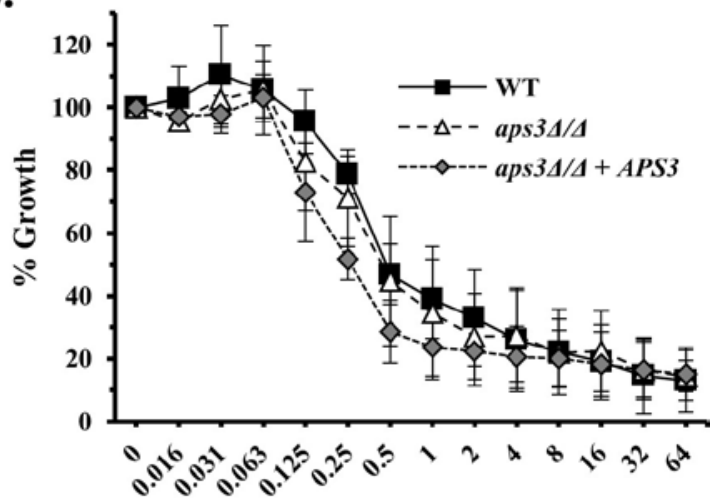

D.

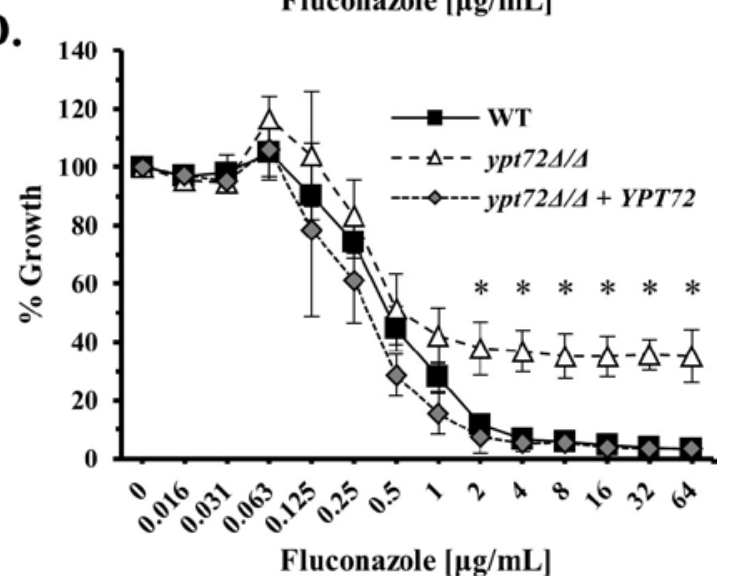

Figure 3-3. Endosomal trafficking defects alter $C$. albicans growth in the presence of fluconazole.

The susceptibility of several C. albicans vacuolar trafficking mutants to fluconazole was compared to 'wild-type' (YJB6284) and 'reconstituted' control strains using the standard CLSI broth microdilution protocol. After 48 hours incubation, growth was measured as $\mathrm{OD}_{600 \mathrm{~nm}}$ and expressed as a percentage of the growth in the minus drug (DMSO alone) control wells. (A) An atg $9 \Delta / \Delta$ mutant blocked in autophagy. (B) An aps $3 \Delta / \Delta$ mutant blocked in a direct Golgi-to-vacuole trafficking pathway. (C) A vps21 $1 \Delta / \Delta$ mutant blocked in membrane fusion and trafficking through the PVC. (D) A ypt72 $\Delta / \Delta$ mutant blocked in membrane fusion at the vacuolar membrane. The mean and standard deviation of three independent experiments are presented in each panel. The growth of each mutant was compared to that of the 'wild-type' control at each drug concentration using a two-tailed $t$-test. $* P<0.05$. 


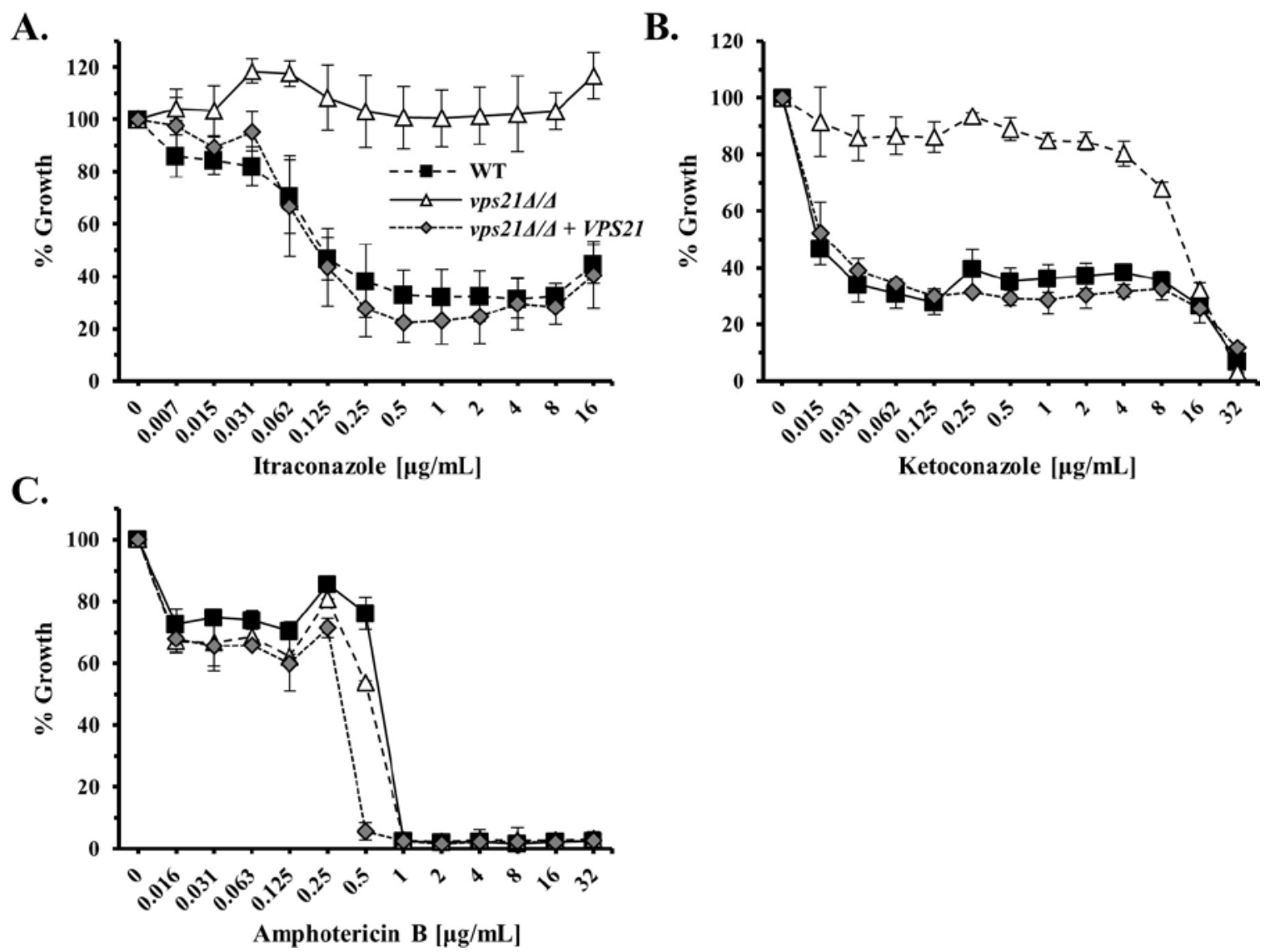

Figure 3-4. The Rab GTPase Vps21p impacts $C$. albicans growth in the presence of the triazole itraconazole and the imidazole ketoconazole.

The $C$. albicans vps $21 \Delta / \Delta$ mutant and isogenic control strains were tested for their susceptibility to (A) itraconazole, (B) ketoconazole, and (C) the polyene amphotericin B using the standard CLSI broth microdilution protocol. After 48 hours incubation, growth was measured as $\mathrm{OD}_{600 \mathrm{~nm}}$ and expressed as a percentage of the growth in the minus drug (DMSO alone) control wells. The mean and standard deviation of two independent experiments are presented in each panel. 
A.

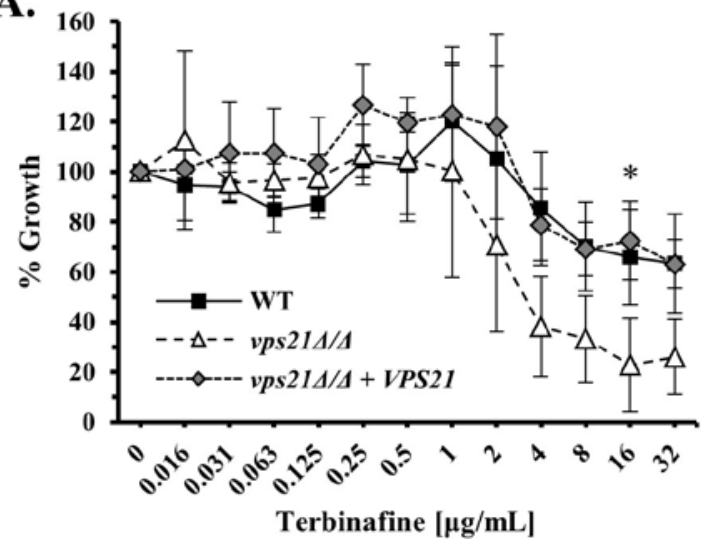

B.

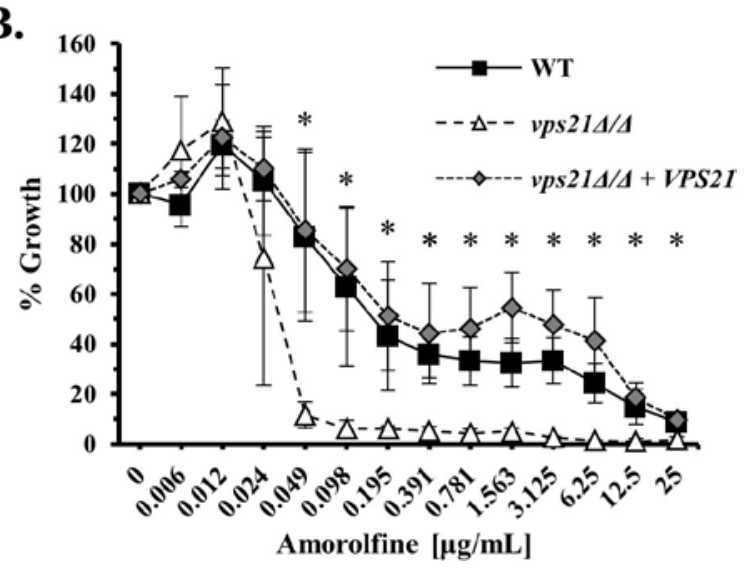

Figure 3-5. Endosomal trafficking impacts $C$. albicans susceptibility to other ergosterol biosynthesis inhibitors.

The susceptibility of the $C$. albicans vps $21 \Delta / \Delta$ mutant to the allylamine terbinafine (A) and the morpholine amorolfine (B) was also determined as described above, except that growth was measured after 24 hours incubation. The mean and standard deviation of three independent experiments are presented in each panel. The growth of each mutant was compared to that of the 'wild-type' control at each drug concentration using a twotailed $t$-test. $* P<0.05$. 
inhibits an earlier step of the ergosterol biosynthetic pathway, and to amorolfine (Figure 3-5B), which acts downstream of Erg11p. Thus the $v p s 21 \Delta / \Delta$ mutants' phenotype seems to be specific to the azoles, rather than a general 'drug resistance' phenotype.

\section{The VPS21 paralogs YPT52 and YPT53 do not significantly affect azole susceptibility}

We recently described two additional GTPases that localize to the PVC, are closely related to, and share significant functional overlap with Vps21p (187). However, the $y p t 52 \Delta / \Delta$ and $y p t 53 \Delta / \Delta$ mutants lacking these GTPases are unaffected in their susceptibility to miconazole (Appendix A, Figure A-1A and B), as is a $y p t 52 \Delta / \Delta y p t 53 \Delta / \Delta$ double mutant (Appendix A, Figure A-1C). While the $y p t 52 \Delta / \Delta$ single mutant has slightly elevated growth in the presence of fluconazole compared to the isogenic control strain (Figure 3-6A), the $y p t 53 \Delta / \Delta$ mutant is unaffected (Figure 3-6B). Furthermore, vps21 $1 \Delta / \Delta y p t 52 \Delta / \Delta$ (Appendix A, Figure A-1E), vps21 $1 \Delta / \Delta y p t 53 \Delta / \Delta$ (Appendix A, Figure A-1F), and a triple GTPase mutant (Figure 3-6C), were all affected by fluconazole to a similar extent as the $v p s 21 \Delta / \Delta$ single mutant. Thus despite significant functional overlap between these three Rab GTPases, it seems that the Vps $21 p$ GTPase has the greatest effect upon C. albicans growth in the presence of the azoles under standard MIC testing conditions.

\section{Susceptibility to azole antifungals is determined by the trafficking step controlled by Vps21p rather than a Vps21p specific function}

To further determine if the growth phenotype shown by the C. albicans vps $21 \Delta / \Delta$ mutant in the presence of the azoles represents a specific function of the Vps21p GTPase, or is a feature of the trafficking step controlled by Vps $21 \mathrm{p}$, we tested the azole susceptibility of a $C$. albicans pep $12 \Delta / \Delta$ mutant (200). Pep12p is a $t$-SNARE protein that is a downstream effector of $\mathrm{Vps} 21 \mathrm{p}$, and also facilitates membrane fusion events at the PVC (202). The growth of the pep $12 \Delta / \Delta$ mutant (Figure 3-7) shows a similar doseresponse to fluconazole as that described above for the vps21 $1 \Delta / \Delta$ mutant (Figure 3-3C), suggesting that this phenotype is a result of a block in PVC transport rather than a specific function of the Vps21p GTPase.

Interestingly, a $C$. albicans vps $28 \Delta / \Delta$ mutant lacking a subunit of the ESCRT I protein sorting complex that controls biogenesis of multivesicular bodies (MVB, which form by invagination of the limiting endosomal membrane for internalization of membrane proteins that are to be sorted to the vacuole) (220) was no more sensitive or tolerant to fluconazole treatment than the control strains (Appendix A, Figure A-2). This underscores the importance of the trafficking step mediated by Vps $21 \mathrm{p}$ in determining azole susceptibility in $C$. albicans over other membrane trafficking events within the PVC. 


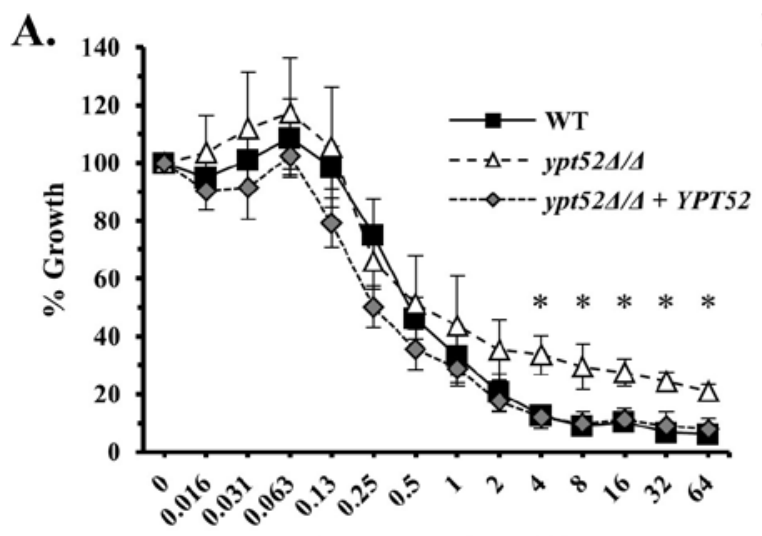

C.

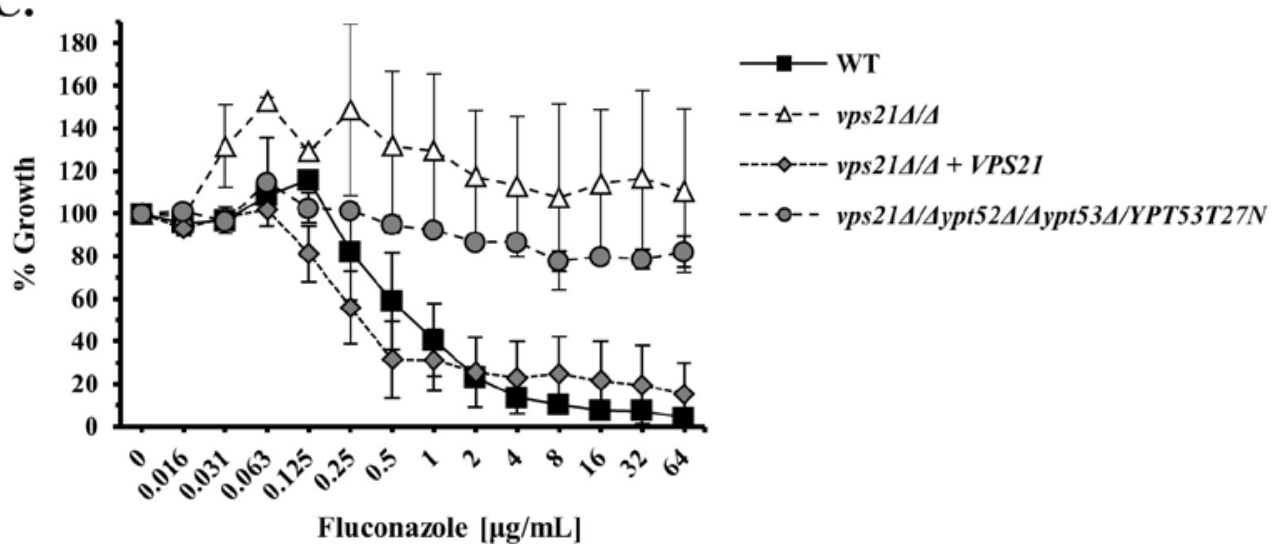

B.

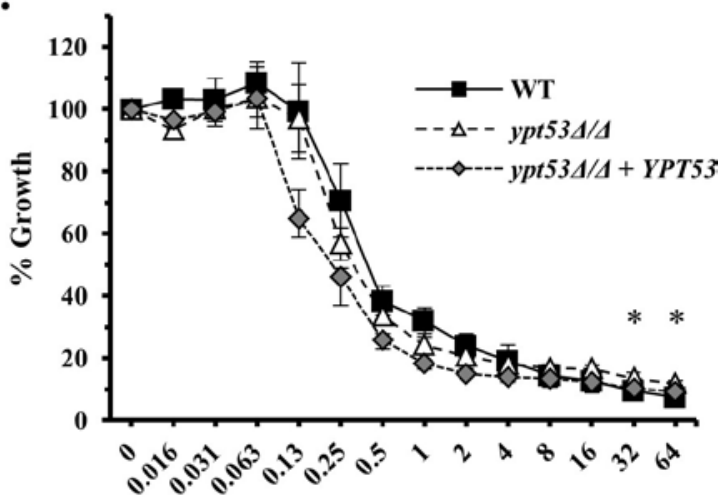

Fluconazole $[\mu \mathrm{g} / \mathrm{mL}]$

Figure 3-6. The Vps21p paralogs Ypt52p and Ypt53p have minimal effect upon $C$. albicans growth in the presence of fluconazole.

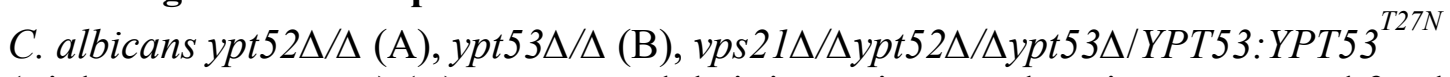
(triple GTPase mutant) (C) mutants and their isogenic control strains were tested for their susceptibility to fluconazole using the standard CLSI broth microdilution protocol. After 48 hours incubation, growth was measured as $\mathrm{OD}_{600 \mathrm{~nm}}$ and expressed as a percentage of the growth in the minus drug (DMSO alone) control wells. The data in each panel is the mean and standard deviation of three independent experiments. The growth of each mutant was compared to that of the 'wild-type' control at each drug concentration using a two-tailed $t$-test. $* P<0.05$. 


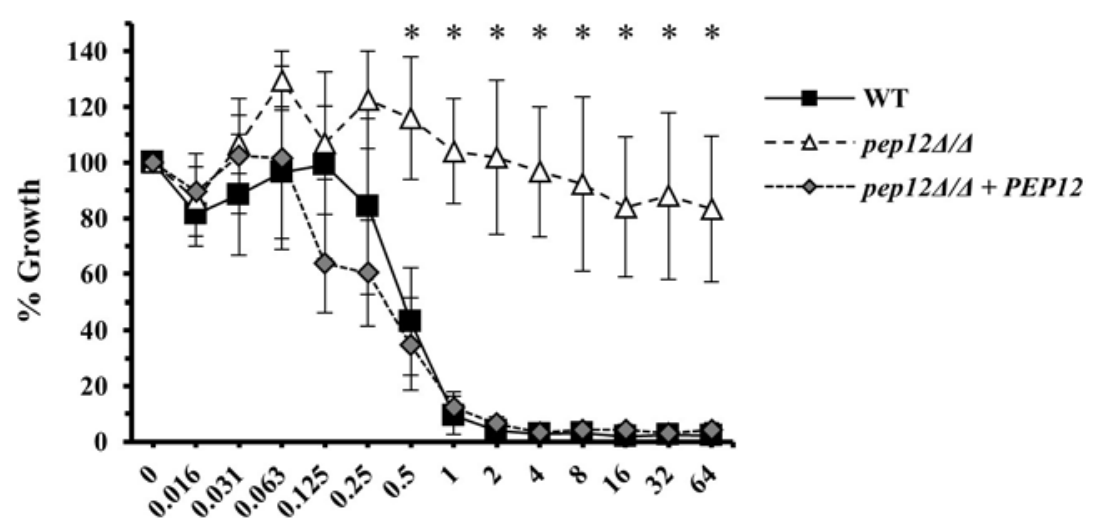

Fluconazole $[\mu \mathrm{g} / \mathrm{mL}]$

Figure 3-7. The $C$. albicans pep $12 \Delta / \Delta$ mutant has reduced susceptibility to fluconazole.

C. albicans pep $12 \Delta / \Delta$ mutants and their isogenic control strains were tested for their susceptibility to fluconazole using the standard CLSI broth microdilution protocol. After 48 hours incubation, growth was measured as $\mathrm{OD}_{600 \mathrm{~nm}}$ and expressed as a percentage of the growth in the minus drug (DMSO alone) control wells. The mean and standard deviation of three independent experiments is shown. The growth of the pep $12 \Delta / \Delta$ mutant was compared to that of the 'wild-type' control at each drug concentration using a two-tailed $t$-test. ${ }^{*} P<0.05$. 


\section{The $C$. albicans vps21 $\triangle \Delta$ mutant exhibits an exaggerated 'trailing growth' phenotype}

The dose-response profiles of the $v p s 21 \Delta / \Delta$ mutant to fluconazole are distinct from that of a well characterized azole resistant isolate TW17 (125) (Figure 3-8A and B). When growth was compared at the earlier time point of 24 hours, it could be discerned that the growth of the $v p s 21 \Delta / \Delta$ mutant was inhibited at similar concentrations of fluconazole to the isogenic control strain, but the degree of growth inhibition was significantly less (Figure 3-9A). In addition, above concentrations of $0.5 \mu \mathrm{g} / \mathrm{mL}$ no further 'dose-dependent' decrease in growth was observed. The differential doseresponse profiles at 24 vs. 48 hours (Figure 3-9A and B) are characteristic of the 'trailing growth' phenomenon (124). Clinical isolates exhibiting 'trailing growth' typically exhibit MIC's of $\leq 1 \mu \mathrm{g} / \mathrm{mL}$ at the 24 hour time point, but $\geq 64 \mu \mathrm{g} / \mathrm{mL}$ at 48 hours. Using a strict cutoff of $80 \%$ growth inhibition ( $\mathrm{MIC}_{80}$ ) the vps $21 \Delta / \Delta$ mutant would be considered azole resistant at both 24 and 48 hour time points. However, adopting a less stringent criterion of $50 \%$ growth inhibition $\left(\mathrm{MIC}_{50}\right.$ ), the $v_{p s} 21 \Delta / \Delta$ mutants' differential response at $24 \mathrm{vs.}$ 48 hours conforms to that of 'trailing growth'. While trailing isolates are generally considered to be susceptible to azole treatment in vivo $(143,144)$, the phenomenon can complicate in vitro antifungal susceptibility testing since it can lead to misclassification of susceptible isolates as resistant ones, impacting therapeutic decisions. Furthermore, the mechanisms underlying trailing growth are not well understood. We therefore examined if the increased growth of the $v p s 21 \Delta / \Delta$ mutant in the presence of the azoles was true resistance, or an exaggerated 'trailing growth' phenotype. It has been previously reported that adjusting the $\mathrm{pH}$ of the RPMI growth medium from 7 to a more acidic $\mathrm{pH}$ is sufficient to eliminate trailing growth, but not true azole resistance (124). At $\mathrm{pH} 3$, the $v p s 21 \Delta / \Delta$ mutant still grew more than the isogenic control strain in the presence of fluconazole (Figure 3-9C), but the differential was substantially diminished. It has also been reported that adjusting the incubation temperature from $35^{\circ} \mathrm{C}$ to $25^{\circ} \mathrm{C}$ or $42^{\circ} \mathrm{C}$ eliminates trailing growth (221). The $v p s 21 \Delta / \Delta$ mutant was completely re-sensitized to fluconazole at either 25 or $42^{\circ} \mathrm{C}$ (Figure 3-9D and $\mathbf{E}$ ), further supporting that the $v p s 21 \Delta / \Delta$ mutants reduced growth inhibition in the presence of the azoles is an exaggerated form of the trailing growth phenomenon, rather than outright resistance.

\section{The vps $21 \Delta \Delta$ mutant is tolerant of Erg11p inhibition and ergosterol depletion compared to control strains}

The dose-response data for the 24 hour time point suggest that the potency of the azoles with respect to $\operatorname{Erg} 11 \mathrm{p}$ inhibition is unchanged in the vps $21 \Delta / \Delta$ mutant, but that the in vitro antifungal efficacy (i.e. impact on growth), is reduced under standard testing conditions. We therefore considered the possibility that the vps $21 \Delta / \Delta$ mutant has a decreased dependence upon ergosterol for growth, or that it was more tolerant than wildtype of the toxic sterol intermediates that accumulate upon azole treatment. To test this we compared cellular ergosterol levels in the vps $21 \Delta / \Delta$ and isogenic control strain, in the presence and absence of fluconazole. This confirmed that both vps $21 \Delta / \Delta$ and control strains are similarly depleted of ergosterol following treatment with fluconazole 
A.

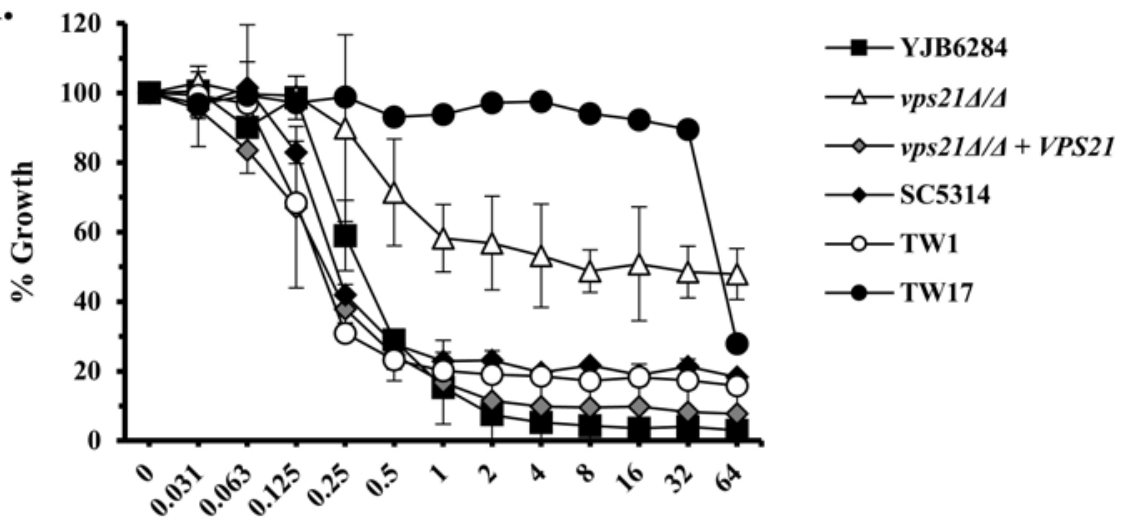

B.

Fluconazole $[\mu \mathrm{g} / \mathrm{mL}], 24 \mathrm{~h}$

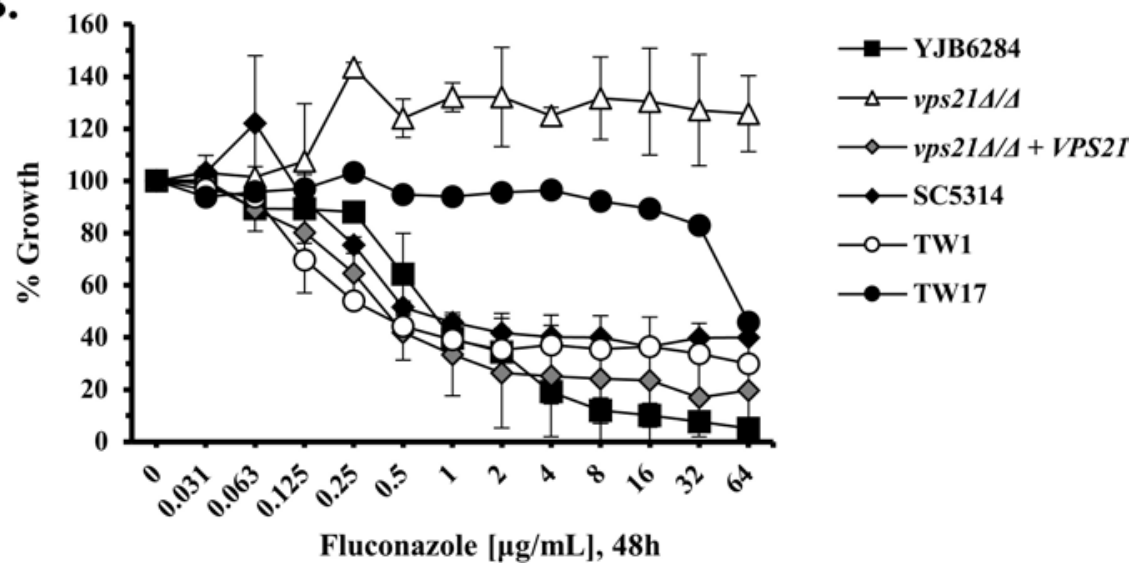

Figure 3-8. The dose-response growth profile of the vps21 $\Delta / \Delta$ mutant to fluconazole is distinct from that of an azole resistant clinical isolate.

A well described, azole susceptible isolate (TW1) and an azole resistant matched strain

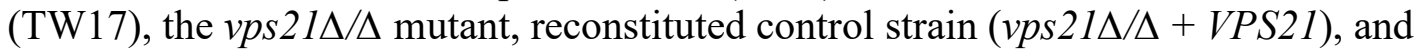
additional "wild-type" control strains (SC5314 and YJB6284) were tested for their susceptibility to fluconazole using the standard CLSI broth microdilution protocol. Growth was determined as $\mathrm{OD}_{600 \mathrm{~nm}}$ after 24 hours (A) and 48 hours (B) incubation and expressed as a percentage of the growth in the minus drug (DMSO) control wells. The means and standard deviations of two biological replicates is shown. 
Figure 3-9. The $C$. albicans vps $21 \Delta / \Delta$ mutant shows features consistent with an exaggerated trailing growth-like phenotype.

The susceptibility of the vps $21 \Delta / \Delta$ mutant and control strains to fluconazole was tested using the standard CLSI protocol and growth compared at either 24 hours (A) or 48 hours (B). The same experiment was also conducted with RPMI medium that was adjusted to a $\mathrm{pH}$ of $3(\mathrm{C})$; or at $\mathrm{pH} 7$ with incubation at either 25 (D) or $42^{\circ} \mathrm{C}(\mathrm{E})$ and growth compared after 48 hours of incubation. Data in panels A-E are the mean and standard deviation of three independent experiments. The growth of the $v p s 21 \Delta / \Delta$ mutant was compared to the WT control at each concentration of fluconazole using a two-tailed $t$-test; ${ }^{*} P<0.05$. (F) The vps $21 \Delta / \Delta$ mutant and isogenic control strains were cultured in YNB broth $\pm 4 \mu \mathrm{g} / \mathrm{mL}$ of fluconazole at $35^{\circ} \mathrm{C}$ for 24 hours, before total cellular sterols were extracted and ergosterol content quantified by NMR analysis. Ergosterol content is expressed as a percentage of the samples wet weight. A C. albicans erg $2 \Delta / \Delta$ mutant (no fluconazole) was used as negative control for ergosterol. (G) Both VPS21 alleles were deleted from the tetO-ERG11 strain. The growth of the resulting vps $21 \Delta / \Delta$, 'reconstituted' and 'wild-type' (YJB6284) control strains was then compared using a broth microdilution assay in YNB medium supplemented with a range of doxycycline concentrations. Growth was measured as $\mathrm{OD}_{600 \mathrm{~nm}}$ and expressed as a percentage of growth occurring in the minus doxycycline control wells. The mean and standard deviation of three independent experiments is indicated, and statistical analysis was performed as described above. 
A.

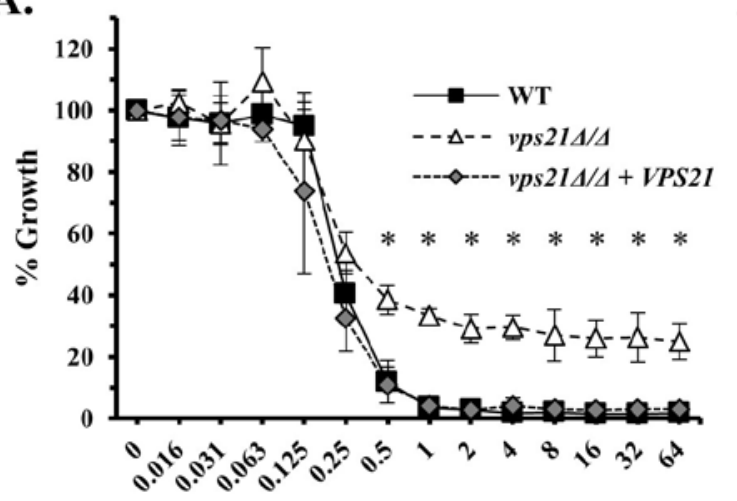

Fluconazole $[\mu \mathrm{g} / \mathrm{mL}]$

pH 7.0, 24 hrs

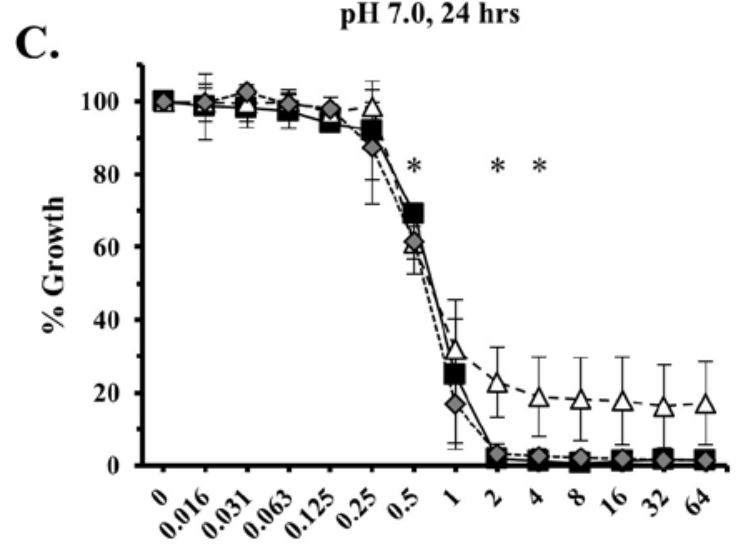

Fluconazole $[\mu \mathrm{g} / \mathrm{mL}]$

E. pH 3.0, 48 hrs

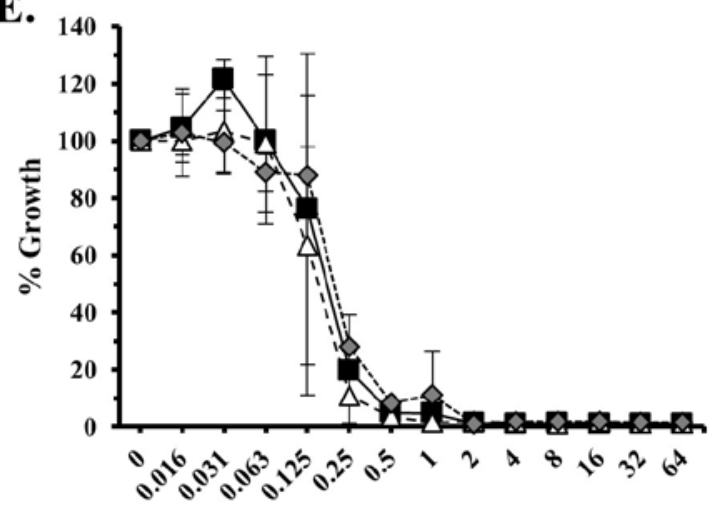

Fluconazole $[\mu \mathrm{g} / \mathrm{mL}]$

G. $42^{\circ} \mathrm{C}$

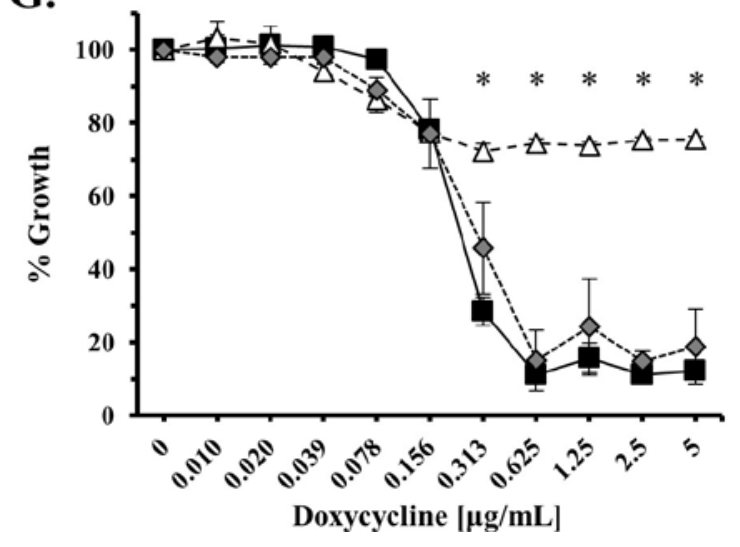

B.

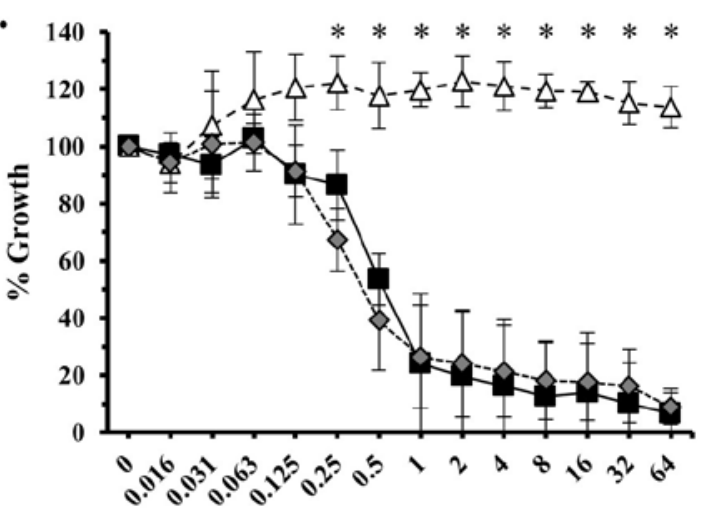

Fluconazole $[\mu \mathrm{g} / \mathrm{mL}]$

D.

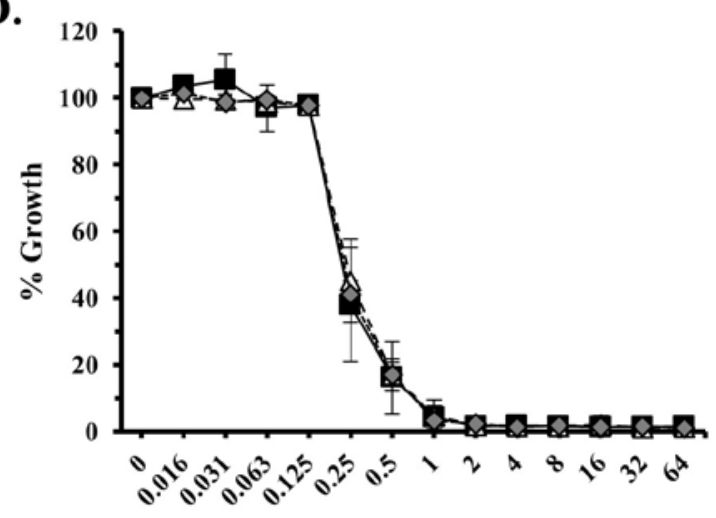

Fluconazole $[\mu \mathrm{g} / \mathrm{mL}]$

F.

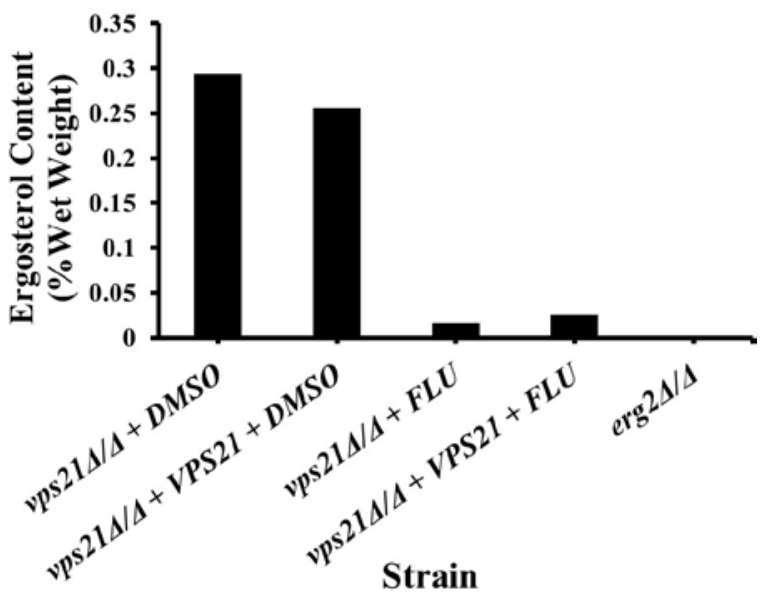

- - $\Delta-$ - tetO-ERG11vps214/A

$\cdots---$ tetO-ERG11vps214/A + VPS2I 
(Figure 3-9F). Furthermore, NMR analysis of cellular n-heptane extracts from mutant and control cells indicates that there are no significant differences in the composition of the sterol intermediates that accumulate upon fluconazole treatment (Appendix A,

Figure A-3A and B). Thus the elevated growth of the $v p s 21 \Delta / \Delta$ mutant is unlikely to be accounted for by altered azole uptake, efflux or target inhibition.

The above results suggest that despite fluconazole inhibiting Erg11p (and ergosterol biosynthesis) to similar extents, the vps $21 \Delta / \Delta$ mutant is able to grow more than its isogenic control, at least under standard MIC testing conditions. To provide further support for this argument, we deleted both VPS21 alleles from the tetO-ERG11 strain described above. This enabled us to repress Erg11p activity at a transcriptional level and independently of the azole drugs. Doxycycline suppressed the growth of the isogenic control strain, but growth was suppressed to a much lesser extent in the vps $21 \Delta / \Delta$ background (Figure 3-9G). Together, these data suggest that the elevated growth of the $v p s 21 \Delta / \Delta$ mutant in the presence of the azoles relates more to a mitigation of the consequences of Erg11p inhibition and ergosterol depletion, rather than a reduction in Erg11p inhibition.

\section{Discussion}

An important finding of this study is that vacuolar trafficking pathways through the PVC are an important determinant of antifungal tolerance. Blocking the PVC trafficking steps controlled by the Rab GTPase Vps21p and $t$-SNARE Pep12p does not seem to affect the azoles capacity to inhibit ergosterol biosynthesis (i.e. potency), but significantly reduces the resulting impact upon fungal growth under standard susceptibility testing conditions $\left(35^{\circ} \mathrm{C}, \mathrm{pH} 7\right)$. The differential dose-response curves at 24 vs. 48 hours, as well as the $\mathrm{pH}$ and temperature dependence of the $v p s 21 \Delta / \Delta$ mutants' decreased susceptibility to the azole antifungals are consistent with it being an exaggerated form of the 'trailing growth' phenotype that has been described for about $18 \%$ of $C$. albicans isolates (145). The mechanism(s) that underlie trailing growth are not well understood, and whether altered endosomal trafficking is a contributing factor to the trailing growth observed in clinical isolates remains to be established. Tests on a limited number of clinical isolates have suggested that the trailing growth phenotype is usually associated with in vivo susceptibility to azole therapy $(143,144)$. Thus we would expect the vps $21 \Delta / \Delta$ and pep $12 \Delta / \Delta$ mutants to be susceptible in vivo. Similarly, it will be important to determine if altered endosomal trafficking contributes to the differences in in vitro as well as in vivo antifungal efficacy that are observed between clinical isolates.

Others have reported that a $C$. albicans vps $15 \Delta / \Delta$ mutant, defective in retrograde trafficking from the PVC back to the Golgi is highly sensitive to fluconazole (222). Furthermore, two mutants lacking components of the ESCRT complex that facilitates the formation of intra-lumenal multi-vesicular bodies from the PVC's surface membrane are also sensitive to fluconazole (223). A further finding of potential clinical relevance is that the vps $21 \Delta / \Delta$ mutant is significantly more susceptible to terbinafine and amorolfine, antifungal medications that target alternate steps within the ergosterol biosynthetic 
pathway. This suggests that membrane trafficking through the PVC compartment is an important determinant of susceptibility to the antifungal agents that target ergosterol biosynthesis. Furthermore, chemical agents that interfere with specific endosomal trafficking steps may sensitize pathogenic fungi, and enhance the antifungal efficacy of therapies that target the ergosterol biosynthetic pathway.

Several major components of sterol biosynthesis are localized to the endoplasmic reticulum (173). While the mechanisms responsible for sterol redistribution to other cellular membranes are poorly understood (224), they are believed to involve both vesicular transport and non-vesicular carrier-protein mediated processes (224-226). Thus one explanation may be that membrane trafficking defects at the PVC could lead to the sub-cellular redistribution of the sterol intermediates that accumulate upon treatment with ergosterol biosynthesis inhibitors. The methylated sterols that accumulate upon azole treatment are thought to contribute to cellular membrane dysfunction and growth inhibition (196). Thus the redistribution of these potentially toxic sterols to distinct compartments within the fungal cell could determine the specific cellular organelles affected, and thus the cellular consequences of antifungal treatment. For example, blocking Vps21p mediated membrane trafficking through the PVC may divert the transport of the 'toxic' sterol intermediates produced within the endoplasmic reticulum upon azole treatment, away from a cellular compartment that is especially sensitive, and redistribute them to a cellular compartment that can better tolerate them. Conversely, the same mechanism renders the $v p s 21 \Delta / \Delta$ mutant more sensitive to the sterol intermediates species that accumulate following treatment with terbinafine or amorolfine. Viewed in this light, the impact of antifungal treatment upon the fungal cell is determined not only by ergosterol depletion, but a combination of the chemical and physical properties of the specific sterol intermediate that accumulates (i.e. which steps of ergosterol are blocked), as well as the cellular destination to which they are transported. 


\section{CHAPTER 4. AZOLE TOLERANCE IN CANDIDA ALBICANS MUTANTS LACKING THE RAB GTPASE VPS21P IS DEPENDENT UPON CALCIUM SIGNALING* $\dagger$}

\section{Introduction}

A form of azole tolerance known as "trailing growth" denotes the persistent growth of some clinical isolates of $C$. albicans and other Candida species, at drug concentrations higher than the minimum inhibitory concentration (MIC). A "trailing" isolate exhibits azole susceptibility at the 24 hour time-point but appears similar to an azole resistant isolate after 48 hours incubation under standard susceptibility testing conditions (144), despite substantial reduction in cellular ergosterol levels $(143,219)$. However, experiments carried out in animal models of mucosal and disseminated candidiasis indicate that strains displaying trailing growth in vitro are susceptible to azoles in vivo $(143,144)$.

The mechanisms underlying trailing growth as well as the biological significance of this phenomenon remain unclear. Some studies have suggested that upregulation of genes related to several azole resistance mechanisms such as ERG11,CDR1,CDR2 and $M D R 1$ in response to azole treatment may play a role in the trailing growth phenomenon (227). However, no direct correlation has been established between ERG11, CDR1 and $M D R 1$ expression levels, or particular amino acid substitutions in Erg11p with the trailing phenomenon (123).

In Chapter 3 we showed that endosomal trafficking is a key determinant of the consequences of ergosterol biosynthesis inhibition, as a $C$. albicans vps $21 \Delta / \Delta$ mutant deficient in membrane fusion events at the PVC is tolerant of the azole antifungals, but more sensitive to exposure to the allylamine terbinafine and morpholine amorolfine. Remarkably, the $v p s 21 \Delta / \Delta$ mutant was able to grow significantly more than the control strains in the presence of the azole antifungals despite ergosterol depletion, a phenotype that resembled an exaggerated form of the trailing growth phenomenon that has been described for some clinical isolates. However, the mechanisms by which the $v p s 21 \Delta / \Delta$ mutant exerts this phenotype are unknown. The objective of the studies described in this

\footnotetext{
* Modified by permission of American Society for Microbiology. Luna-Tapia A, Kerns ME, Eberle KE, Jursic BS, Palmer GE. 2015. Trafficking through the late endosome significantly impacts Candida albicans tolerance of the azole antifungals. Antimicrob Agents Chemother 59:2410-2420. doi:10.1128/AAC.04239-14.

$\uparrow$ Modified by permission of American Society for Microbiology. Luna-Tapia A, Tournu H, Peters TL, Palmer GE. 2016. Endosomal trafficking defects can induce calcium dependent azole tolerance in Candida albicans. Antimicrob Agents Chemother 60:000 000. doi:10.1128/AAC.01034-16.
} 
chapter is to define the mechanisms underlying the azole tolerance of the vps $21 \Delta / \Delta$ mutant.

\section{Materials and Methods}

\section{Growth conditions}

C. albicans was routinely grown in YPD medium (1\% yeast extract, $2 \%$ peptone, $2 \%$ dextrose) at $30^{\circ} \mathrm{C}$, supplemented with uridine $(50 \mu \mathrm{g} / \mathrm{mL})$ when necessary. Transformant selection was carried out on minimal YNB medium $(6.75 \mathrm{~g} / \mathrm{L}$ yeast nitrogen base without amino acids, $2 \%$ dextrose, $2 \%$ Bacto agar), supplemented with the appropriate auxotrophic requirements as described for $S$. cerevisiae (185) or $50 \mu \mathrm{g} / \mathrm{mL}$ uridine.

\section{Plasmid construction}

Plasmid pLUX (213), was kindly provided by Dr. William Fonzi (Georgetown University). Plasmids pLUXVPS21 (51), pKE1 (187), and pKE1-NLUC (172) were previously described. All oligonucleotides used in this study are listed in Table 4-1.

The VPH1 ORF with 5' and 3' UTR sequences was amplified from SC5314 genomic DNA (gDNA) with HiFi Platinum Taq (Invitrogen) and primer set VPH1AMPF + VPH1AMPR-KpnI, and cloned between the $S a c I$ and KpnI restriction sites of pLUX to produce plasmid pLUXVPH1.

pLUXCDR1 was produced by amplifying the CDR1 ORF with 5' and 3' untranslated region sequences from SC5314 genomic DNA (gDNA) with HiFi Platinum Taq (Invitrogen) and primer set CDR1AMPF2 + CDR1AMPR2, and then cloned between the NsiI and $A g e I$ restriction sites of pLUX.

For construction of the reporter plasmid pRTA2prGFP $\gamma(228), 1000 \mathrm{bp}$ of the RTA2 promoter were amplified from SC5314 gDNA with HiFi Platinum Taq (Invitrogen) and primer pairs RTA2prF-KpnI + RTA2prR-SalI, and cloned between the KpnI and SalI sites of pKE1 in place of the $A C T 1$ promoter. The GFP $\gamma$ coding sequence was then amplified using GFPAMPF-SalI + GFPAMPR-MluI, and cloned downstream of the RTA2 promoter between $S a l \mathrm{I}$ and $M l u \mathrm{I}$ sites.

\section{C. albicans strain construction}

Strains used in this study are listed in Table 4-2. Transformation of C. albicans with DNA constructs was performed using the lithium acetate method (217). Gene deletion strains were constructed by the PCR-based approach described by Wilson et al. 
Table 4-1. List of oligonucleotides used in the study described in Chapter 4.

\begin{tabular}{|c|c|}
\hline Primer & Sequence (5'-3') \\
\hline ARG4DETF & ATCAATTAACACAGAGATACC \\
\hline ARG4DETR & CCGAGCTTGGCGTAATCATGG \\
\hline ARG4INTF2 & AAGCTAGTGTGGAAAGAAGAG \\
\hline ARG4INTR2 & AATGACTGAATTATGTCGGTC \\
\hline & ATATCACATATATATTCTATTTATTTTTTGTACTTAATAAT \\
\hline CDR1DISF & $\begin{array}{l}\text { TTCTTTAAAAGGTCAAAAACGAAAAAATTGTTTTCCCAGT } \\
\text { CACGACGTT }\end{array}$ \\
\hline & ATAGTCTAAAAACGTCTATTATATTTTAGACGTTTGAGAT \\
\hline CDR1DISR & $\begin{array}{l}\text { ACCACCATGTCAAAAAACAAACTGTTTAATTGTGGAATT } \\
\text { GTGAGCGGATA }\end{array}$ \\
\hline CDR1AMPF2 & GTAGCAAGTGTGTCAAGAACC \\
\hline CDR1AMPR2 & TCAACCGGTCAGATGAGAAACACTTTTTCCC \\
\hline CDR1DETF2 & GGAGCTCAACGGAAAATTGCC \\
\hline CDR1DETF3 & GGTGCCATGACTCCTGCTACCG \\
\hline CDR1DETR2 & AGATGAGAAACACTTTTTCCC \\
\hline CDR1DETR3 & CACCAGCGACACCAACTAGGGC \\
\hline GFPAMPF-SalI & TCAGTCGACATGTCTAAAGGTGAAGAATTATTC \\
\hline GFPAMPR-MluI & TCAACGCGTTTATTTGTACAATTCATCC \\
\hline HIS1DETF & TCATCCTCCAGGATCCCGCGG \\
\hline HIS1DETR & TGTCACCTAAATAGCTTGGCG \\
\hline HIS1INTR2 & CGACCATATGGGAGAGCTCCC \\
\hline LUXINTDETF & CTGACCTTTAGTCTTTCCTGC \\
\hline LUXINTDETR & CAGTAGTACTTGTTGTTGTATCG \\
\hline MDR1DISF & $\begin{array}{l}\text { TTCCGTAACAATCATATTATAATTTTACATTGCCCCAATA } \\
\text { GCAATACATATACTTACATAGAACTTCATAGTTTTCCCAG } \\
\text { TCACGACGTT }\end{array}$ \\
\hline MDR1DISR & $\begin{array}{l}\text { CTTTTCTCTTTTTAATTATTGATTAATGTATCTATAACACG } \\
\text { ATATATCTATAGGAAAACAATGACACCTCTGTGGAATTGT } \\
\text { GAGCGGATA }\end{array}$ \\
\hline MDR1AMPF & TCAGGATCCTCACACGAACAAAAACAACCC \\
\hline MDR1AMPR & TCAGGATCCTAATGAGAACATTGTGTCCGCG \\
\hline MDR1DETF & AATAGTTGTCACTTGGGATG \\
\hline MDR1DETR & AGCACCCAAACTCCAAGCGGC \\
\hline RTA2prF-KpnI & TCAGGTACCGAATGTTAACTAAGGGGTGG \\
\hline RTA2prR-SalI & TCAGTCGACAGTTGAAGGGTGGGAATGAACTG \\
\hline URA3INTF & TTAGTGTTACGAATCAATGGC \\
\hline URA3INTF2 & TTATACCATCCAAATCCCGCG \\
\hline URA3INTF2 & TTATACCATCCAAATCCCGCG \\
\hline URA3INTR & CAATTATAAATGTGAAGGGGG \\
\hline URA3INTR2 & CAAGAAGAAGGATTTGATTGGC \\
\hline
\end{tabular}


Table 4-1. (Continued).

\begin{tabular}{ll}
\hline \multicolumn{1}{c}{ Primer } & \multicolumn{1}{c}{ Sequence (5'-3') } \\
\hline & CAACGACTAAAAGATAACCGTCATTCTCTTCACTAATATA \\
VPH1DISF & CTTCTGTTCCCCCCCCTCTCTCTTATCCCTGTGGAATTGT \\
& GAGCGGATA \\
& GCTTTAATGAATGAATCTAAACAAGTTAAAAACCCCAAC \\
VPH1DISR & TCTATGGTTCTAGGCTTATAAAACACTGTCTGTTTCCCA \\
& GTCACGACGTT \\
VPH1AMPF & TCTGGAGCTCCGTATCTGCACC \\
VPH1AMPR-KpnI TCAGGTACCCAATATTTATTGACATTGCTAGGG \\
VPH1DETF & CACAATTAGAAAGTGGTGGTGCC \\
VPH1DETF2 & CGGAAACAATGTCATCTATGG \\
VPH1DETR & CAACATATTTAATAATCCTGGTGG \\
VPH1DETR2 & GGATGAATAATCTCGATGAGC \\
\hline
\end{tabular}


Table 4-2. List of strains used in the study described in Chapter 4.

\begin{tabular}{|c|c|c|}
\hline Strain & Relevant Genotype & Reference \\
\hline SC5314 & Wild-type/clinical isolate & $(229)$ \\
\hline CAI4 & $u \mathrm{ra} 3 \Delta / \Delta$ & $(193)$ \\
\hline BWP17 & $\operatorname{ura} 3 \Delta / \Delta$ his $1 \Delta / \Delta \arg 4 \Delta / \Delta$ & $(190)$ \\
\hline YJB6284 & ura3 $\Delta / \Delta: U R A 3$ his $1 \Delta / \Delta: H I S 1 \arg 4 \Delta / \Delta: A R G 4$ & $(215)$ \\
\hline$v p s 21 \Delta / \Delta$ & ura3 $\Delta / \Delta: U R A 3$ his $1 \Delta / \Delta \arg 4 \Delta / \Delta$ vps $21 \Delta: H I S 1 / v p s 21 \Delta: A R G 4$ & $(51)$ \\
\hline $\operatorname{vps} 21 \Delta / \Delta+V P S 21$ & ura3 $\Delta / \Delta: U R A 3: V P S 21$ his $1 \Delta / \Delta \arg 4 \Delta / \Delta$ vps $21 \Delta: H I S 1 / v p s 21 \Delta: A R G 4$ & $(51)$ \\
\hline$c d r 1 \Delta / \Delta$ & ura3 $\Delta / \Delta: U R A 3$ his $1 \Delta / \Delta \arg 4 \Delta / \Delta \operatorname{cdrl} 1 \Delta: H I S 1 / c d r 1 \Delta: A R G 4$ & This study \\
\hline$c d r l \Delta / \Delta+C D R 1$ & 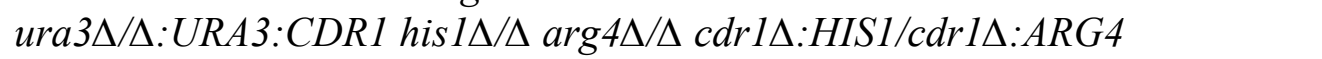 & This study \\
\hline$v p s 21 \Delta / \Delta c d r 1 \Delta / \Delta$ & 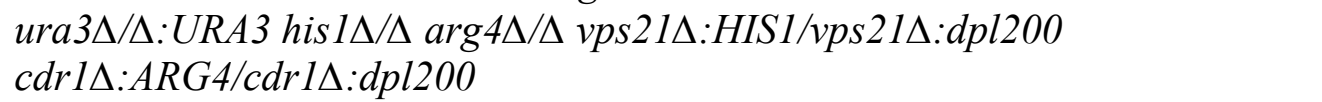 & This study \\
\hline$v p s 21 \Delta / \Delta c d r 1 \Delta / \Delta+\operatorname{VPS} 21$ & 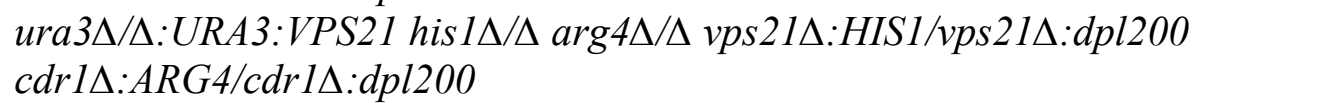 & This study \\
\hline$v p s 21 \Delta / \Delta c d r 1 \Delta / \Delta+C D R 1$ & 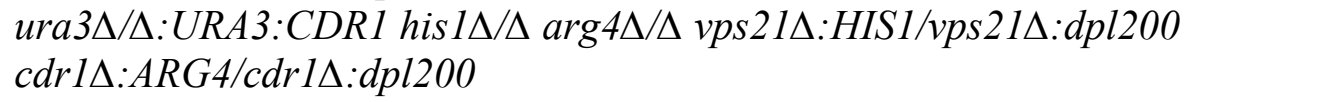 & This study \\
\hline$v p h 1 \Delta / \Delta$ & 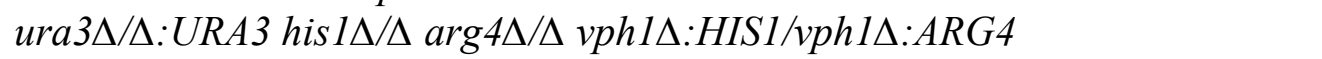 & This study \\
\hline$v p h 1 \Delta / \Delta+V P H 1$ & 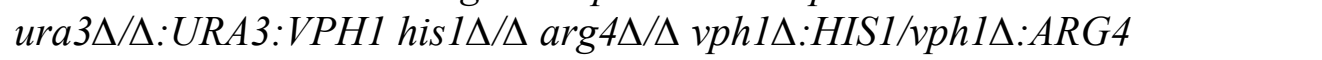 & This study \\
\hline$v p s 21 \Delta / \Delta v p h 1 \Delta / \Delta$ & 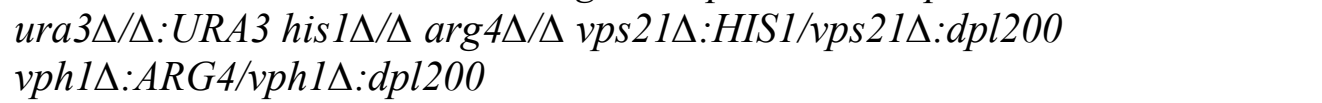 & This study \\
\hline$v p s 21 \Delta / \Delta v p h 1 \Delta / \Delta+V P S 21$ & 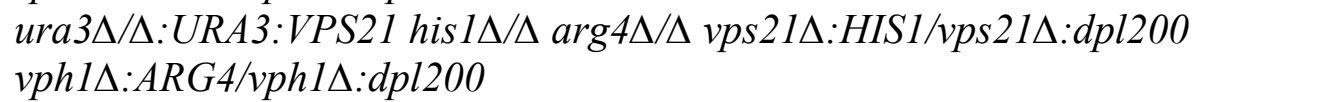 & This study \\
\hline$v p s 21 \Delta / \Delta v p h 1 \Delta / \Delta+V P H 1$ & 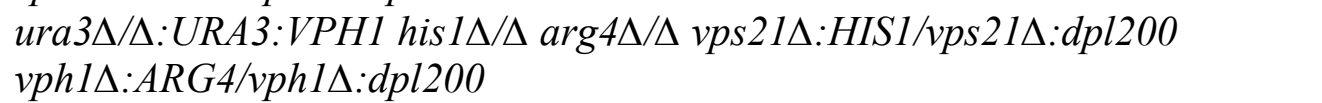 & This study \\
\hline $\mathrm{CAI} 4+A C T 1 p r N L U C$ & ura3 $\triangle / \triangle: U R A 3: A C T 1 p r N L U C$ & This study \\
\hline $\operatorname{vps} 21 \Delta / \Delta+A C T 1 p r N L U C$ & ura3 $\Delta / \Delta: U R A 3: A C T 1 p r N L U C$ his $1 \Delta / \Delta \arg 4 \Delta / \Delta$ vps $21 \Delta: H I S 1 / v p s 21 \Delta: A R G 4$ & This study \\
\hline $\mathrm{CAI} 4+R T A 2 p r G F P \gamma$ & ura3 $\Delta / \Delta: U R A 3: R T A 2 p r G F P \gamma$ & This study \\
\hline$v p s 21 \Delta / \Delta+R T A 2 p r G F P \gamma$ & 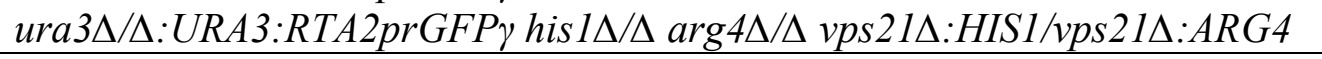 & This study \\
\hline
\end{tabular}


(190) using the $u r a 3 \Delta / \Delta$ his $1 \Delta / \Delta \arg 4 \Delta / \Delta$ strain BWP17 (kindly provided by Dr. Aaron Mitchell, Carnegie Mellon University). Strain CAI4 was kindly provided by Dr. William Fonzi (Georgetown University).

The vps $21 \Delta / \Delta$, aps $3 \Delta / \Delta$, and vps $21 \Delta / \Delta a p s 3 \Delta / \Delta$ mutants were constructed in previous studies $(50,51,187,214)$. Control strain YJB6284 (215) was kindly provided by Dr. Judith Berman (Tel Aviv University).

CDR1 deletion cassettes were amplified by PCR with HiFi Platinum Taq (Invitrogen) and primers CDR1DISF and CDR1DISR, using pRS-ARG4 $\triangle$ SpeI, pGEMHIS1, or pDDB57 (containing a recyclable URA3-dpl200 marker) $(190,230)$ as templates. Each $C D R 1$ allele was sequentially deleted by using HISI and $A R G 4$ markers to generate $c d r 1 \Delta / \Delta u r a 3 \Delta / \Delta$ gene deletion mutants. Correct integration of deletion cassettes was confirmed at each step by PCR with the following primers sets: ARG4DETF + CDR1DETF2 and ARG4DETR + CDR1DETR2 (ARG4 integration), or HIS1DETF + CDR1DETF2 and HIS1DETR + CDR1DETR2 (HIS1 integration). The absence of an intact $C D R 1$ allele was confirmed using primer pair CDR1DETF3 + CDR1DETR3. Finally, isogenic mutant and $C D R 1$ reconstituted strains were produced by transforming the $c d r 1 \Delta / \Delta u r a 3 \Delta / \Delta$ mutant with either pLUX (vector alone) or pLUXCDR1 following restriction digestion with NheI. Correct integration of the pLUX vector fully restores the $U R A 3$ and adjacent $I R O 1$ loci, and this was confirmed by PCR analysis using primer pair LUXINTDETF + LUXINTDETR which yields a 2199 bp product following restoration of the URA3 and IROI loci.

A vps $21 \Delta / \Delta c d r 1 \Delta / \Delta$ double mutant was also made from a vps $21 \Delta / \Delta$ ura $3 \Delta / \Delta \arg 4 \Delta / \Delta$ strain previously described (51). The first allele of $C D R 1$ was deleted with the URA3-dpl200 selection marker and the second allele with the $A R G 4$ marker. Replacement of the first CDR1 allele with the URA3-dpl200 selection marker was confirmed using primer pairs URA3INTF + CDR1DETF2 and URA3INTR + CDR1DETR2. The resulting vps $21 \Delta / \Delta c d r 1 \Delta / \Delta$ double mutant was then selected on YNB medium supplemented with uridine and $1 \mu \mathrm{g} / \mathrm{mL} 5$-FOA (192) to select for $\mathrm{ura3}^{-}$ segregants that had excised the $U R A 3$ marker gene. Correct excision of the URA3 marker from the $c d r 1 \Delta: U R A 3-d p l 200$ loci was confirmed using the flanking primer pairs CDR1DETF2 and CDR1DETR2. Prototrophic and isogenic mutant and reconstituted control strains were then made by transforming the vps $21 \Delta / \Delta c d r 1 \Delta / \Delta$ double mutant with NheI digested pLUX, pLUXVPS21 (51), and pLUXCDR1. Correct integration of the plasmids and reconstitution of the URA3 and IROI loci was confirmed as described above.

The MDRI ORF was deleted using an identical strategy to that described above for $C D R 1$, except the deletion cassettes were amplified with primers MDR1DISF and MDR1DISR. Correct integration of the MDR1 deletion cassettes was confirmed using primer pairs ARG4DETF + MDR1AMPF and ARG4DETR + MDR1AMPR (ARG4 integration), HIS1DETF + MDR1AMPF and HIS1DETR + MDR1AMPR (HIS1 integration), or URA3INTF + MDR1AMPF and URA3INTR + MDR1AMPR (URA3 integration). Deletion of both $M D R 1$ alleles was also confirmed by the absence of a PCR 
product using primer pair MDR1DETF + MDR1DETR which binds to sequences within the deleted portion of $M D R 1$. Prototrophic and isogenic mutant and reconstituted control strains were then made by transforming the $m d r 1 \Delta / \Delta$ mutant and vps $21 \Delta / \Delta m d r 1 \Delta / \Delta$ double mutant with NheI digested pLUX. Correct plasmid integration and restoration of $U R A 3$ and IROI loci was determined as previously described.

VPH1 deletion cassettes were amplified by PCR with primers VPH1DISF + VPH1DISR, using pRS-ARG4 $\triangle$ SpeI, pGEM-HIS1, or pDDB57 (containing a recyclable URA3-dpl200 marker) $(190,230)$ as templates. Each $V P H 1$ allele was sequentially deleted using $H I S 1$ and $A R G 4$ markers to generate $v p h 1 \Delta / \Delta u r a 3 \Delta / \Delta$ gene deletion mutants (228). Correct integration of the deletion cassettes was confirmed at each step by PCR with the following primers sets: ARG4INTF2 + VPH1DETR2 and ARG4INTR2 + VPH1DETF2 for $A R G 4$ integration or HIS1INTR2 + VPH1DETF2 for HIS1 integration. The absence of an intact $V P H 1$ allele was confirmed using primer pair VPH1DETF + VPH1DETR. Isogenic mutant and $V P H 1$ reconstituted strains were produced by transforming the vph $1 \Delta / \Delta u r a 3 \Delta / \Delta$ mutant with either $\mathrm{pLUX}$ (vector alone) or $\mathrm{pLUXVPH1} \mathrm{after}$ linearization with NheI. Correct plasmid integration and restoration of URA3 and IROI loci was determined as described above.

Construction of a $v p s 21 \Delta / \Delta v p h 1 \Delta / \Delta$ double mutant from a vps $21 \Delta / \Delta u r a 3 \Delta / \Delta \arg 4 \Delta / \Delta$ strain previously described (51) was carried out by deleting the first allele of $V P H 1$ with the URA3-dpl2O0 selection marker and the second allele with the ARG4 marker (228). Replacement of the first VPH1 allele with the URA3-dpl200 selection marker was confirmed using primer pairs URA3INTF2 + VPH1DETR2 and URA3INTR2 + VPH1DETF2. The resulting vps $21 \Delta / \Delta v p h 1 \Delta / \Delta$ double mutant was then selected on YNB-agar plates supplemented with uridine and 5-fluoroorotic acid (5FOA)(192) to select for $u \mathrm{ra}^{-}$segregants that had excised the $U R A 3$ marker gene. Correct excision of the URA3 marker from the vph1A:URA3-dpl200 loci was confirmed using the flanking primer pair VPH1AMPF + VPH1AMPR-KpnI. Prototrophic and isogenic mutant and reconstituted control strains were then made by transforming the $v p s 21 \Delta / \Delta v p h 1 \Delta / \Delta$ double mutant with pLUX, pLUXVPS21, or pLUXVPH1 digested with NheI (228). Correct plasmid integration and restoration of URA3 and IROI loci was determined as previously described.

C. albicans strains expressing cytoplasmic Nano Luciferase ${ }^{\circledR}$ (Nluc) (231) (produced with permission from Promega Corporation), were produced using the previously described pKE1-NLUC plasmid (172), which was linearized using NheI, and transformed into $\mathrm{ura}^{-}$recipient strains. Strains carrying the $R T A 2-G F P \gamma$ reporter were made by transforming CAI4 and vps21 $\Delta / \Delta u r a 3^{-}$strains with the NheI digested pRTA2prGFP $\gamma$. Correct plasmid integration and restoration of $U R A 3$ and IROI loci was determined as described above. 


\section{Luciferase based membrane integrity assay}

C. albicans strains expressing a cytoplasmic version of Nano Luciferase (Nluc) (231) were cultured overnight in YPD at $30^{\circ} \mathrm{C} .200 \mu \mathrm{l}$ of each culture was then transferred to an Eppendorf tube and the cells pelleted using a microfuge. The culture supernatant was then removed, the cells resuspended in $1 \mathrm{~mL}$ YNB and further diluted 1:100 in YNB medium. $200 \mu \mathrm{L}$ of each cell suspension was then dispensed into the wells of a round bottomed 96 -well plate. After 24 hour incubation in a $30^{\circ} \mathrm{C}$ or $35^{\circ} \mathrm{C}$ standing incubator, the cells in each well were resuspended by mixing using a multichannel pipette. The plates were then centrifuged at $1200 \mathrm{rpm}$ in a benchtop centrifuge to pellet the cells. $50 \mu \mathrm{L}$ of the culture supernatant was then transferred to a white flat-bottomed 96 well plate and Nluc activity determined using the Nano-Glo® Luciferase Assay Reagent (Promega Corporation), as per the manufacturer's instructions. Luminescence was then measured using a Cytation ${ }^{\mathrm{TM}} 5$ plate reader (BioTek Instruments, Inc.). Growth in each well from the overnight incubation was also determined by measuring OD $_{600 \mathrm{~nm}}$ from samples diluted 1:10 in distilled water using a separate flat bottomed 96-well plate, and each luminescence reading normalized to the corresponding $\mathrm{OD}_{600 \mathrm{~nm}}$ reading.

\section{Biomass measurement and determination of cell viability}

C. albicans strains were grown overnight in $\mathrm{YNB}$ at $30^{\circ} \mathrm{C}$, washed and subcultured into $50 \mathrm{~mL}$ of fresh YNB at $2 \times 10^{6}$ cells $/ \mathrm{mL}$, in the presence of $5 \mu \mathrm{g} / \mathrm{mL}$ of fluconazole or $0.5 \%$ DMSO and incubated at 30 or $35^{\circ} \mathrm{C}$ for 24 hours with shaking. Cell viability was then determined as colony forming units (CFU) from culture samples following appropriate dilution in YNB and plating to YPD agar plates. $40 \mathrm{~mL}$ of each culture was also pelleted at $3500 \mathrm{rpm}$, washed by resuspension in $5 \mathrm{~mL}$ of deionized $\mathrm{H}_{2} \mathrm{O}$, and harvested by filtration using a preweighed $0.22 \mu \mathrm{m}$ GS filter membrane (Millipore). Cell pellets were dehydrated at $65^{\circ} \mathrm{C}$ for 24 hours and dry weight determined (228).

\section{Antifungal susceptibility testing}

Antifungal susceptibility testing of all the strains was performed using the broth micro-dilution method described in the CLSI document M27-A3 (218) in a 96-well plate format. All drugs for susceptibility testing used here were diluted in DMSO in 2-fold dilutions of 200 times the final concentration. RPMI 1640 medium (Sigma-Aldrich) was prepared according to the CLSI document (218), and the medium was buffered with morpholinepropanesulfonic acid (MOPS) and $\mathrm{pH}$ adjusted to 7.0 using $\mathrm{NaOH}$. Plates were incubated without shaking at $35^{\circ} \mathrm{C}$ for 24 or 48 hours unless otherwise stated. The content of each well was carefully resuspended by pipetting up and down before OD $_{600 \mathrm{~nm}}$ was measured using a Cytation ${ }^{\mathrm{TM}} 5$ plate reader (BioTek Instruments, Inc.). Checkerboard assays with fluconazole (Sigma-Aldrich) and the calcineurin inhibitors CsA (Sigma-Aldrich) or FK506 (Tacrolimus, Tocris) were performed in a 96-well plate in MOPS-buffered RPMI 1640 pH 7.0 according to the CLSI standard. Susceptibility 
testing using combinations of fluconazole $(1 \mu \mathrm{g} / \mathrm{mL})$ plus nisoldipine $(0.39-50 \mu \mathrm{M})$ or ethylene glycol tetraacetic acid (EGTA, $50-500 \mu \mathrm{M}$ ) was performed as described above. Plates were incubated and growth measured as described before.

\section{Determination of intracellular free $\mathrm{Ca}^{2+}$ levels}

The relative levels of intracellular free $\mathrm{Ca}^{2+}$ were determined by using the $\mathrm{Ca}^{2+}$ sensitive dye Fura 2-AM (EMD Millipore Corporation). C. albicans strains were grown overnight in YPD at $30^{\circ} \mathrm{C}$, washed in PBS, and approximately $\sim 10^{7}$ cells resuspended in $200 \mu \mathrm{L}$ PBS pH 7.2 (without $\mathrm{CaCl}_{2}$ and $\mathrm{MgCl}_{2}$ ) plus $10 \mu \mathrm{M}$ Fura 2-AM. Each cell suspension was then dispensed into the wells of a round bottomed 96-well plate. Following incubation at $37^{\circ} \mathrm{C}$ for 1 hour, cells were pelleted and washed three times with PBS. Fluorescence was determined by dual excitation at $340 \mathrm{~nm}$ and $380 \mathrm{~nm}$ with a single emission at $505 \mathrm{~nm}$ in a Cytation ${ }^{\mathrm{TM}} 5$ plate reader (BioTek Instruments, Inc.) (228) with the relative $\mathrm{Ca}^{2+}$ levels expressed as the ratio of fluorescence intensities upon excitation at $380 \mathrm{~nm}$ and $340 \mathrm{~nm}(232)$.

\section{Quinacrine accumulation assay}

Quinacrine is a weak base that accumulates in acidic membrane organelles such as the fungal vacuole in yeast (233) and is used as a qualitative measurement of organelle acidification. The unprotonated form of quinacrine enters the cell and becomes protonated upon entry into an acidic organelle, accompanied by a boost in its fluorescence intensity (234). Labeling of the $C$. albicans vacuole with quinacrine was performed as described by Baggett et al (233) with the following modifications. $10 \mathrm{mM}$ quinacrine stocks were prepared in $1 \mathrm{M}$ HEPES $\mathrm{pH} 7.6$ and stored at $-20^{\circ} \mathrm{C}$ until use. Cells were grown overnight in YPD medium at $30^{\circ} \mathrm{C}$ in a rotator. Approximately $5 \times 10^{7}$ cells per sample of each strain were harvested by centrifugation at $8000 \mathrm{rpm}$ for 30 seconds and resuspended in $100 \mu \mathrm{L}$ of assay buffer (100 mM HEPES plus $2 \%$ glucose). Quinacrine was added to one set of samples at a final concentration of $25 \mu \mathrm{M}$; an equivalent volume of 1 M HEPES pH $7.6+2 \%$ glucose was added to a second set of samples to determine cell autofluorescence. A minus cell control containing assay buffer and $25 \mu \mathrm{M}$ quinacrine was included to determine quinacrine binding to plastic. Samples were incubated during 5 minutes at room temperature in the dark; then were pelleted at $8000 \mathrm{rpm}$ for 30 seconds and cells resuspended in $100 \mu \mathrm{L}$ of fresh assay buffer without quinacrine and placed on ice until use. $100 \mu \mathrm{L}$ of each sample were transferred to a black, flat bottom 96-well plate. Three samples per set per biological replicate were used. 100 $\mu \mathrm{L}$ of assay buffer alone was added to three additional wells to determine medium background. Fluorescence intensity was determined in a Cytation ${ }^{\mathrm{TM}} 5$ Cell Imaging Multi-Mode Reader (BioTek Instruments, Inc.). Excitation and emission were set at 490 $\mathrm{nm}$ and $525 \mathrm{~nm}$, respectively, with a $9 \mathrm{~nm}$ bandwidth. Net fluorescence intensity was normalized to the $\mathrm{OD}_{600 \mathrm{~nm}}$ of each sample and expressed as a fold change from the fluorescence intensity of the WT control strain (YJB6284). 


\section{GFP $\gamma$ reporter assays}

C. albicans strains expressing GFP $\gamma$ (235) from the RTA2 promoter were grown overnight in YPD at $30^{\circ} \mathrm{C}$. Cells were then washed and resuspended in YNB and $\sim 1.3 \mathrm{x}$ $10^{6}$ cells per well were dispensed into a round bottomed 96-well plate. Cells were treated with either $1 \mu \mathrm{g} / \mathrm{mL}$ fluconazole or $0.5 \% \mathrm{DMSO}$, and incubated at $35^{\circ} \mathrm{C}$ for 24 hours. GFP $\gamma$ fluorescence intensity was then quantified using a Cytation ${ }^{\mathrm{TM}} 5$ plate reader (BioTek Instruments, Inc.) with excitation at $488 \mathrm{~nm}$ and emission at $507 \mathrm{~nm}$. Growth was determined by measuring $\mathrm{OD}_{600 \mathrm{~nm}}$ for normalization of the fluorescence signal.

\section{Results}

\section{The $C$. albicans vps $21 \Delta / \Delta$ mutant is tolerant of azole induced membrane damage}

We previously showed that the vps $21 \Delta / \Delta$ mutant exhibits enhanced growth in the presence of the azole antifungals versus WT controls, despite ergosterol depletion. We therefore examined plasma membrane integrity following azole mediated ergosterol depletion, to determine if the vps $21 \Delta / \Delta$ mutant exhibited signs of membrane damage. A cytoplasmic version of Nano Luciferase (Nluc - Promega Corp.) (231) was expressed in mutant and control strains, and Nluc release into the culture supernatant was determined following treatment with fluconazole for 24 hours. Interestingly, fluconazole treatment at $35^{\circ} \mathrm{C}$, caused significant Nluc release from the WT strain indicating loss of plasma membrane integrity (Figure 4-1A). However, at this temperature the vps $21 \Delta / \Delta$ mutant released significantly less Nluc upon fluconazole treatment than WT, indicating less membrane damage. In contrast, at $30^{\circ} \mathrm{C}$ the mutant released similar levels, or even slightly more Nluc than WT (Figure 4-1B), indicating that fluconazole induces similar levels of membrane permeabilization in either strain at the lower temperature. This is consistent with our previous observation that the continued growth of the vps $21 \Delta / \Delta$ mutant in the presence of the azoles (as measured by $\mathrm{OD}_{600 \mathrm{~nm}}$ ), occurs at $35^{\circ} \mathrm{C}$ but not $30^{\circ} \mathrm{C}$.

To further confirm the temperature dependence of the vps $21 \Delta / \Delta$ mutant's azole tolerance, we compared its growth in the presence and absence of fluconazole using two additional measures, dry weight and colony forming units (Figure 4-1C and D). By both measures, at $35^{\circ} \mathrm{C}$ the $v p s 21 \Delta / \Delta$ mutant was more tolerant of fluconazole than WT, while at $30^{\circ} \mathrm{C}$ the growth of both strains was severely inhibited. As such, it appears that at $35^{\circ} \mathrm{C}$ the azole antifungals cause less membrane damage to the vps $21 \Delta / \Delta$ mutant than WT, and this likely accounts for its continued growth. 
A.

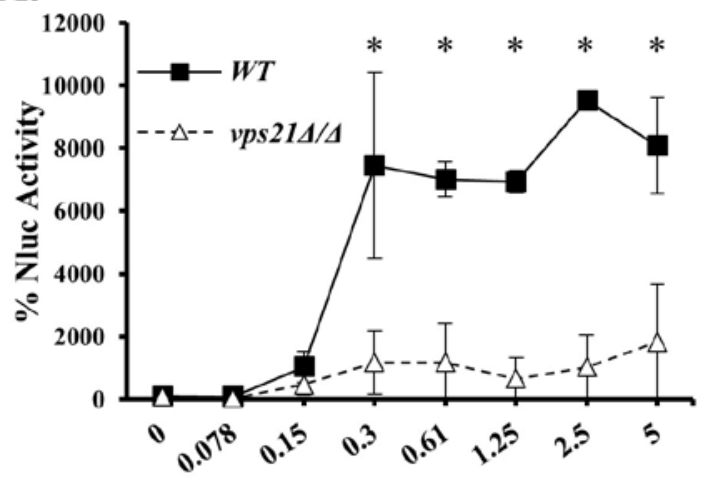

Fluconazole $[\mu \mathrm{g} / \mathrm{mL}] ; 35^{\circ} \mathrm{C}$

C.

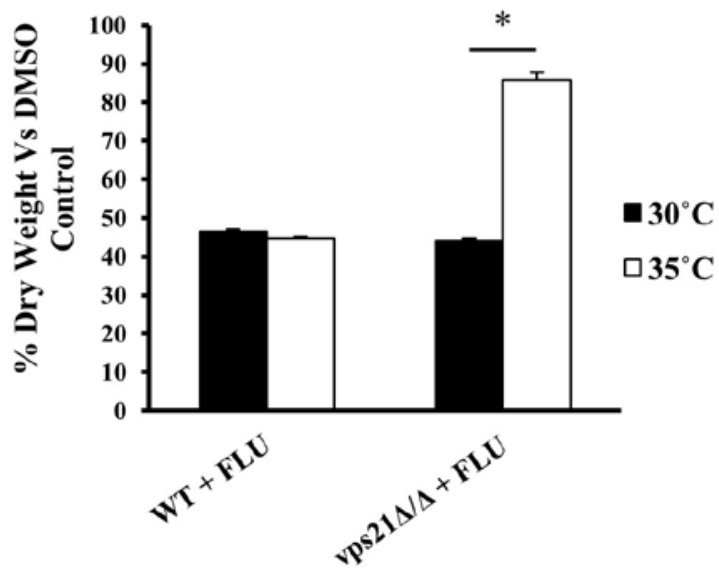

B.

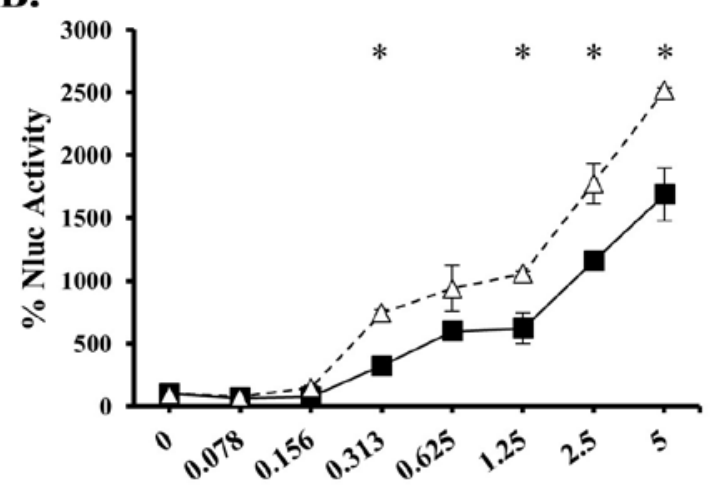

Fluconazole $[\mu \mathrm{g} / \mathrm{mL}] ; 30^{\circ} \mathrm{C}$

D.

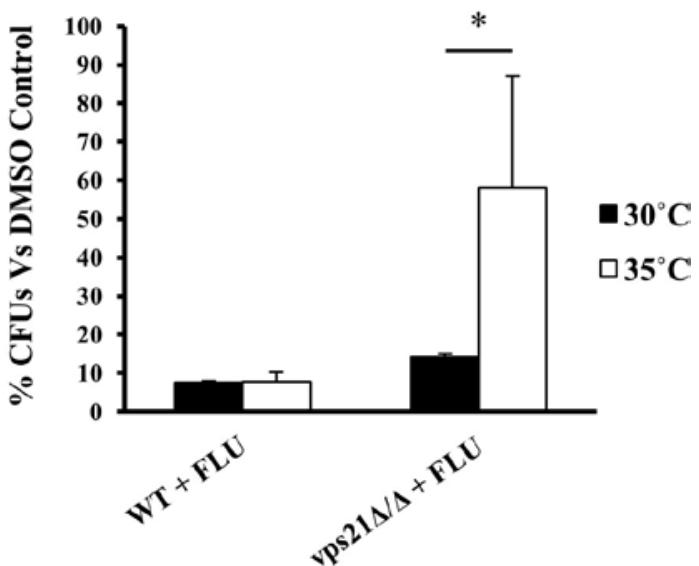

Figure 4-1. The $C$. albicans vps $21 \Delta / \Delta$ mutant has delayed membrane damage following azole treatment.

Nluc was expressed in the vps $21 \Delta / \Delta$ mutant and wild-type (CAI4) control strain, which were grown in 96-well plates with various concentrations of fluconazole. Culture supernatant was removed after 24 hours of incubation at (A) $35^{\circ} \mathrm{C}$ or (B) $30^{\circ} \mathrm{C}$, Nluc activity assayed, and expressed as a percentage of the minus drug controls. The means of three biological replicates are shown for each drug concentration, and error bars indicate the standard deviation. The means of each group were compared using a two-tailed $t$-test, $* P<0.05$. (C-D) The vps $21 \Delta / \Delta$ mutant and WT were grown in YNB at $2 \times 10^{6}$ cells $/ \mathrm{mL}$ in the presence of $0.5 \%$ DMSO or $5 \mu \mathrm{g} / \mathrm{mL}$ of fluconazole (FLU) for 24 hours at $30^{\circ} \mathrm{C}$ or $35^{\circ} \mathrm{C}$. Biomass as measured by $(\mathrm{C})$ dry weight and cell viability as measured by (D) CFUs were determined and expressed as a percentage of the DMSO control for each strain under each condition. The means \pm standard deviations of two biological replicates are shown for all panels. The means of each group were compared using a two-tailed $t$ test, $* P<0.05$. 


\section{Azole tolerance in the $C$. albicans vps21 $1 \Delta$ mutant does not depend upon Vph1p function}

A recent study has proposed that inhibition of the V-ATPase proton pump may in part underlie the antifungal activity of the azoles (79). In yeast, delivery of the V-ATPase to the vacuolar membrane depends on trafficking through the PVC. A S. cerevisiae vps 214 mutant is deficient in the delivery of the V-ATPase to vacuole, and thus its ability to acidify the vacuole is impaired, as evidenced by decreased accumulation of the weak base quinacrine within the fungal vacuole (236). We therefore determined whether vacuolar acidification was affected in our $v p s 21 \Delta / \Delta$ mutant. Unexpectedly, our $v p s 21 \Delta / \Delta$ mutant accumulated more quinacrine than the isogenic control strains, indicating a more acidified vacuole (Figure 4-2A). In order to determine whether the altered vacuolar acidification within the vps $21 \Delta / \Delta$ mutant contributed to its azole tolerance, we deleted both alleles of VPH1, a gene encoding for a vacuole-specific subunit of the V-ATPase (184). However, loss of VPHI does not affect the susceptibility of the 'wild-type' control strain to fluconazole, or the trailing growth phenotype of the vps $21 \Delta / \Delta$ mutant (Figure 42B). These data demonstrate that the azole tolerance of the vps $21 \Delta / \Delta$ mutant does not depend upon V-ATPase activity.

\section{Azole tolerance in the $C$. albicans vps $21 \Delta \Delta$ mutant is independent of the Cdr1p and Mdr1p efflux pumps}

Many plasma membrane proteins are targeted for degradation within the vacuole following endocytic trafficking via the PVC. We have previously reported that the $C$. albicans vps $21 \Delta / \Delta$ mutant has reduced rates of endocytosis (51). Thus we considered that reduced rates of uptake and degradation, may increase the 'half-life' of the well described Cdrlp and Mdrlp efflux pumps at the cell surface, and the resulting increase in abundance of these pumps may contribute to the tolerance of the vps $21 \Delta / \Delta$ mutant. To test this we deleted both alleles of either $C D R 1$ or $M D R 1$ from the $v p s 21 \Delta / \Delta$ mutant and examined the azole susceptibility of the resulting vps $21 \Delta / \Delta c d r 1 \Delta / \Delta$ and $v p s 21 \Delta / \Delta m d r 1 \Delta / \Delta$ double mutants. Loss of MDR 1 had no impact on the MIC of either the 'wild-type' control or the vps $21 \Delta / \Delta$ mutant (not shown) as it is not typically expressed in susceptible strains (140). While loss of CDRl caused a slight increase in azole susceptibility in the WT, it did not significantly affect the susceptibility of the vps 21 $\Delta / \Delta$ mutant (Figure 4-3). These results suggest that the azole tolerance of the vps $21 \Delta / \Delta$ mutant does not depend upon the activity of either the Mdr1p or Cdr1p efflux pumps.

\section{The $C$. albicans $v p s 21 \Delta / \Delta$ mutant has elevated cytoplasmic calcium levels}

$\mathrm{Ca}^{2+}$ based signaling has been shown to induce adaptive responses that promote C. albicans survival following azole treatment $(147,237)$. Moreover, intracellular $\mathrm{Ca}^{2+}$ is compartmentalized in different organelles, with the fungal vacuole being the major storage site (238). To determine if the vacuolar trafficking defects of the vps $21 \Delta / \Delta$ 
A.

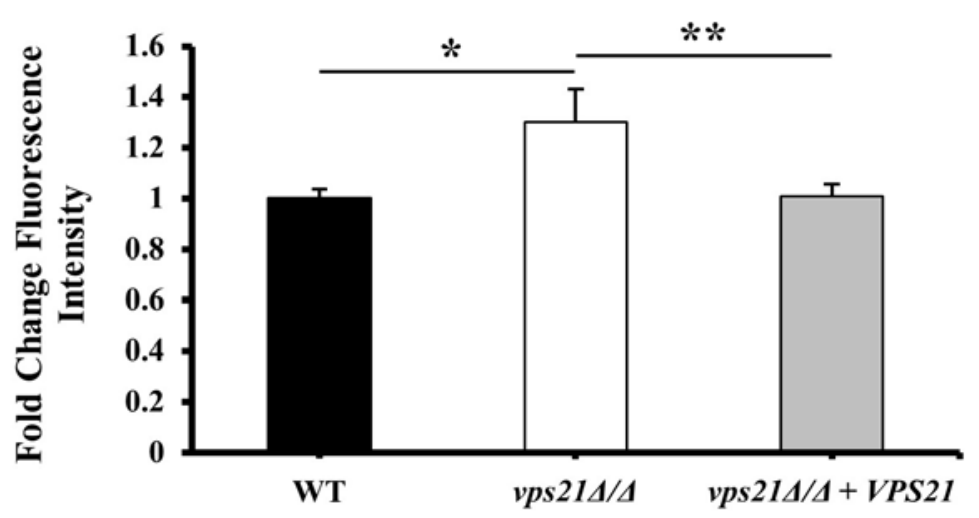

B.

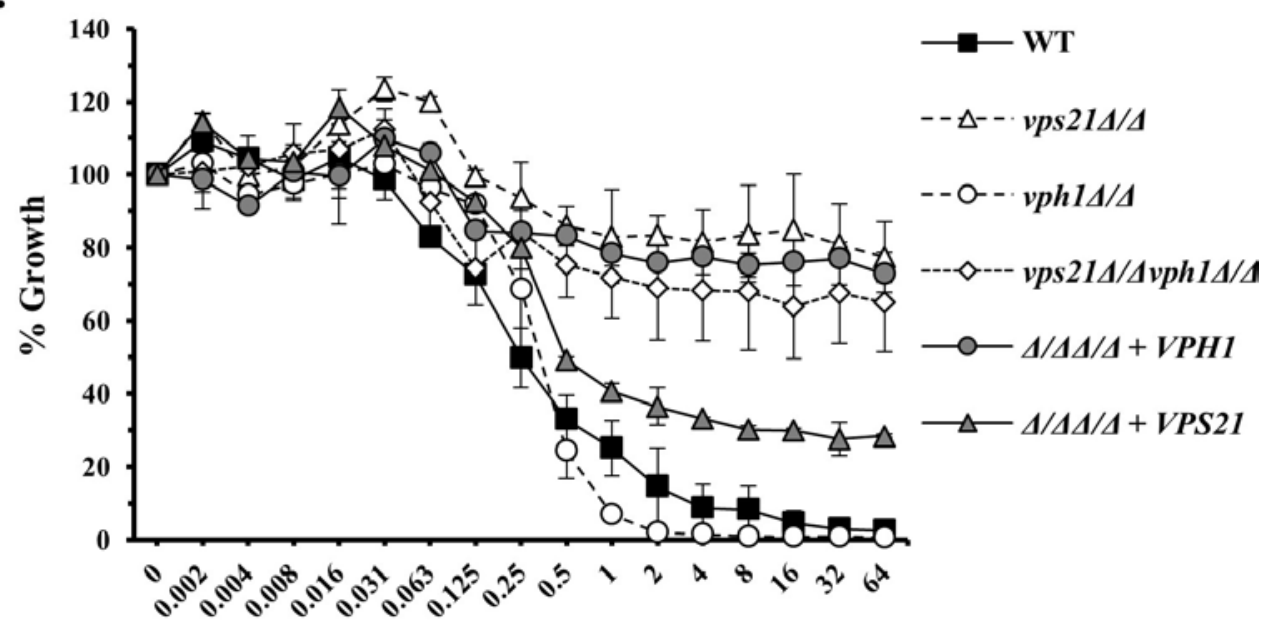

Fluconazole $[\mu \mathrm{g} / \mathrm{mL}]$

Figure 4-2. Deletion of gene encoding for the V-ATPase subunit Vph1p from the $v p s 21 \Delta / \Delta$ mutant does not restore sensitivity to azole antifungals.

(A) Quinacrine uptake in the vps $21 \Delta / \Delta$ mutant and control strains was measured from overnight cultures in YPD. The mean \pm standard deviation of four biological replicates is shown. The means of each group were compared using a two-tailed $t$-test, ${ }^{*}, P<0.005$; $* *, P<0.006$. (B) $V P H 1$, encoding a vacuole specific subunit of the V-ATPase proton pump, was deleted from either wild-type or the C. albicans vps21 $1 \Delta / \Delta$ mutant, and the fluconazole susceptibility of each strain was compared to 'wild-type' (YJB6284), vps $21 \Delta / \Delta$ and 'reconstituted' control strains using the standard CLSI broth microdilution protocol each strain tested using the standard CLSI broth microdilution protocol. Following 48 hours incubation, growth was measured as $\mathrm{OD}_{600 \mathrm{~nm}}$ and expressed as a percentage of the growth in the minus drug (DMSO alone) control wells. The means \pm standard deviations of two biological replicates are shown. No statistically significant differences were found between the growth of the $v p s 21 \Delta / \Delta$ and the $v p s 21 \Delta / \Delta v p h 1 \Delta / \Delta$ mutants. 


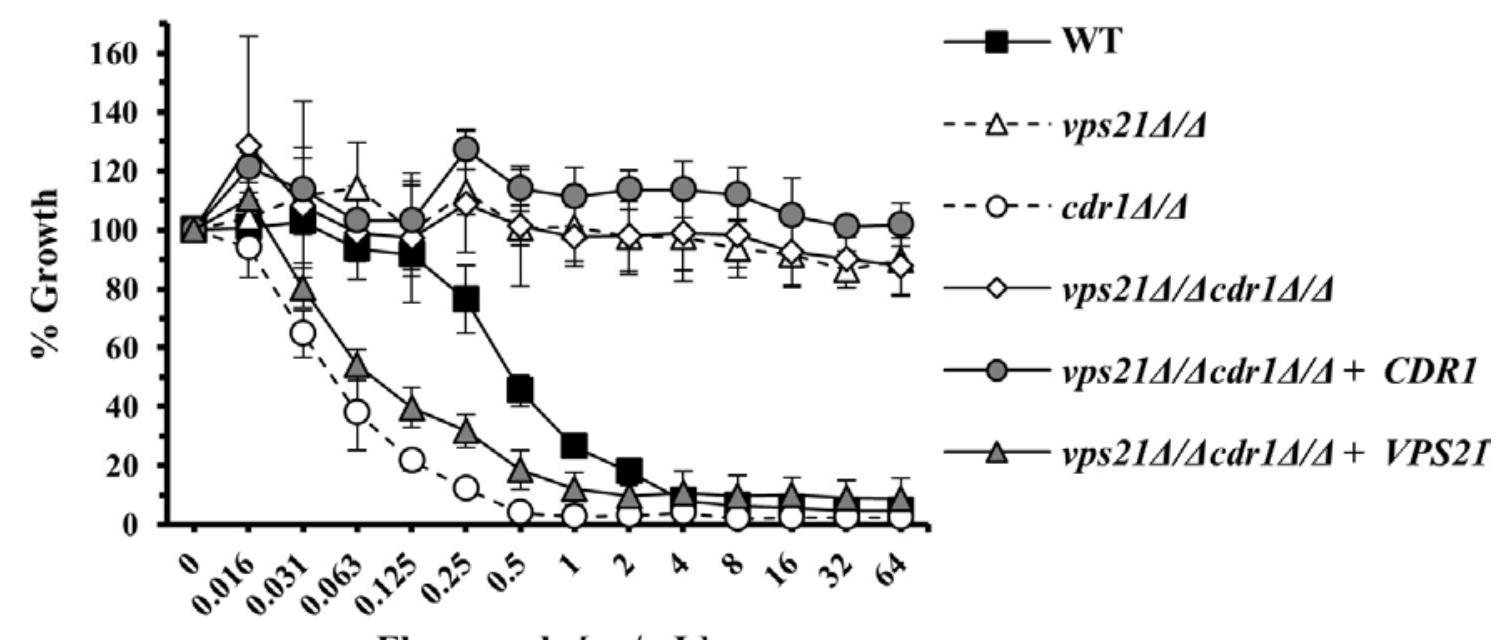

Fluconazole $[\mu \mathrm{g} / \mathrm{mL}]$

Figure 4-3. Growth of the $C$. albicans vps $21 \Delta / \Delta$ mutant in the presence of fluconazole does not depend upon the Cdr1p efflux pump.

Both alleles of the CDR1 gene were deleted from either 'wild-type' (YJB6284) or the $v p s 21 \Delta / \Delta$ mutant and the susceptibility of the resulting strains to fluconazole tested using the standard CLSI broth microdilution protocol. Following 48 hours incubation, growth was measured as $\mathrm{OD}_{600 \mathrm{~nm}}$ and expressed as a percentage of the growth in the minus drug (DMSO alone) control wells. The mean and standard deviation of three independent experiments is presented. No statistically significant differences were found between the growth of the $v p s 21 \Delta / \Delta$ and the $v p s 21 \Delta / \Delta c d r 1 \Delta / \Delta$ mutants. 
mutant impact cellular $\mathrm{Ca}^{2+}$ homeostasis, we measured levels of cytoplasmic calcium with the calcium-sensitive dye Fura 2-AM (232). As expected, the antiarrhythmic drug amiodarone (AMD), known to cause cytoplasmic $\mathrm{Ca}^{2+}$ surges in $S$. cerevisiae (239), increased intracellular free $\mathrm{Ca}^{2+}$ levels in our wild-type control strain (Figure 4-4A). The relative intracellular calcium levels for the vps $21 \Delta / \Delta$ mutant were higher than for the WT controls (Figure 4-4B) as indicated by higher $380 \mathrm{~nm} / 340 \mathrm{~nm}$ fluorescence intensity ratios. This indicates that the $C$. albicans vps $21 \Delta / \Delta$ mutant has a calcium homeostasis defect.

\section{The $C$. albicans vps $21 \Delta / \Delta$ mutant's azole tolerance is dependent on extracellular calcium}

Azole tolerance in C. albicans and other fungi has been shown to be reliant on $\mathrm{Ca}^{2+}$ dependent signaling $(147,237)$, which in turn relies on the availability of both extracellular and intracellular free $\mathrm{Ca}^{2+}(152)$. We therefore examined the dependence of the vps $21 \Delta / \Delta$ mutant's azole tolerance on extracellular $\mathrm{Ca}^{2+}$ levels. Addition of $1 \mathrm{mM}$ $\mathrm{CaCl}_{2}$ to the medium did not significantly enhance the growth of both $v p s 21 \Delta / \Delta$ mutant and control strains in the presence of fluconazole (Figure 4-5A). However, the $\mathrm{Ca}^{2+}$ chelator EGTA reduced the mutant's tolerance to fluconazole (Figure 4-5B and C), establishing a requirement for extracellular $\mathrm{Ca}^{2+}$ for its azole tolerance. Entry of extracellular $\mathrm{Ca}^{2+}$ into the fungal cells depends on several $\mathrm{Ca}^{2+}$ channels located at the plasma membrane including the voltage-gated calcium channel formed by Mid1p/Cch1p $(161,240)$, a high-affinity $\mathrm{Ca}^{2+}$ influx system. Treatment of the $v p s 21 \Delta / \Delta$ mutant with the voltage-gated $\mathrm{Ca}^{2+}$ channel blocker nisoldipine (Figure 4-5E) also reduced its azole tolerance, at concentrations far below that at which nisoldipine is toxic by itself (Figure 4-5D). This further supports the conclusion that the vps $21 \Delta / \Delta$ mutant depends on extracellular $\mathrm{Ca}^{2+}$ in order to tolerate azole exposure.

In further experiments we evaluated the impact of the $\mathrm{Ca}^{2+}$ homeostasis defect of the vps $21 \Delta / \Delta$ mutant by characterizing the effect on azole tolerance of AMD, known to cause cytoplasmic $\mathrm{Ca}^{2+}$ surges due to an increase in $\mathrm{Ca}^{2+}$ fluxes from the media and intracellular stores (239) (Figure 4-5F and G). Surprisingly, under standard MIC testing conditions, AMD dramatically enhanced growth of the WT control strain in the presence of fluconazole (Figure 4-5G). In contrast, growth of the $v p s 21 \Delta / \Delta$ mutant in the presence of fluconazole was largely unresponsive to AMD (Figure 4-5G). This further confirms a $\mathrm{Ca}^{2+}$ homeostasis defect in the vps $21 \Delta / \Delta$ mutant and highlights the role of $\mathrm{Ca}^{2+}$ fluxes in azole tolerance in C. albicans in general.

\section{The $C$. albicans $v p s 21 \Delta / \Delta$ mutant's azole tolerance is dependent upon calcineurin signaling}

Signaling through the $\mathrm{Ca}^{2+}$-responsive phosphatase calcineurin has been shown to be required for azole tolerance in C. albicans (147), as pharmacologic or genetic 
A.

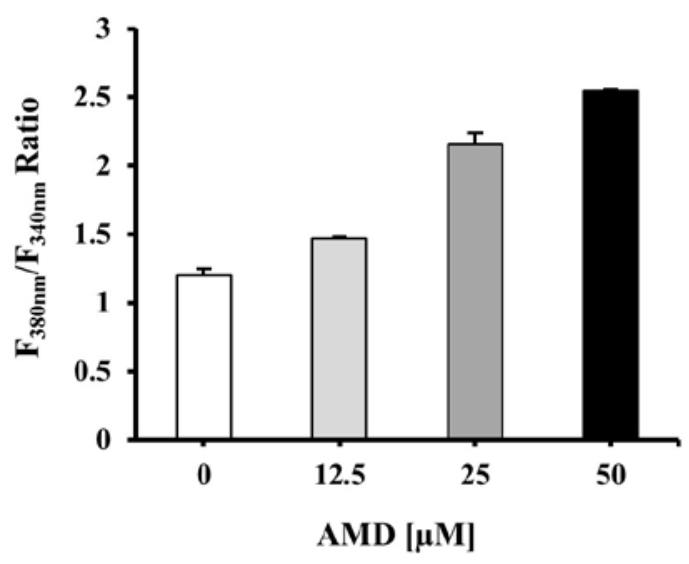

B.

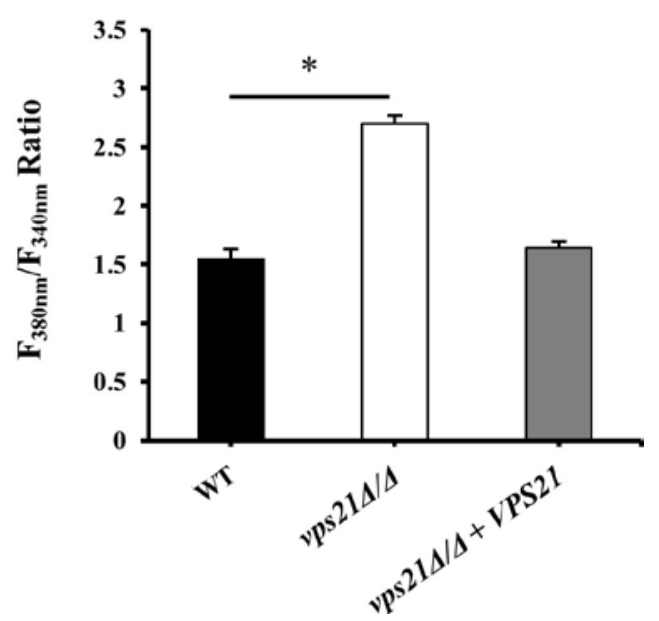

Figure 4-4. The C. albicans vps21 $\Delta / \Delta$ mutant has elevated intracellular levels of $\mathrm{Ca}^{2+}$.

(A) The control strain SC5314 was treated with a range on concentrations of AMD for 2 hours in order to induce $\mathrm{Ca}^{2+}$ fluxes into the cytoplasm, followed by staining with the $\mathrm{Ca}^{2+}$ sensitive dye Fura 2-AM. Fluorescent intensity with dual excitation and $340 \mathrm{~nm}$ and $380 \mathrm{~nm}$ and single emission at $505 \mathrm{~nm}$ was measured in Cytation ${ }^{\mathrm{TM}} 5$ multi-mode reader. Relative $\mathrm{Ca}^{2+}$ levels are expressed as the ratio of the fluorescence intensity at $380 \mathrm{~nm}$ and $340 \mathrm{~nm}$. The mean \pm standard deviation of two experimental replicates from a representative experiment is shown. (B) Free intracellular levels of $\mathrm{Ca}^{2+}$ were measured in the vps $21 \Delta / \Delta$ mutant and control strains using Fura 2-AM. WT is strain YJB6284. Fluorescence was determined as described above. The relative intracellular levels of $\mathrm{Ca}^{2+}$ are expressed as the ratio of the fluorescence intensity upon excitation at $380 \mathrm{~nm}$ and 340 $\mathrm{nm}$. The means \pm standard deviations of four biological replicates are shown. The means of each group were compared using a two-tailed $t$-test, ${ }^{*} P<0.0001$. 
Figure 4-5. The $C$. albicans vps21 $\Delta / \Delta$ mutant's azole tolerance depends upon extracellular calcium.

(A) The C. albicans vps21 $\Delta / \Delta$ mutants' susceptibility to fluconazole was tested after addition of $1 \mathrm{mM}$ of $\mathrm{CaCl}_{2}$ to the media, and compared to wild-type (YJB6284), and reconstituted control strains using the standard CLSI broth microdilution protocol. After 48 hours incubation, growth was measured as $\mathrm{OD}_{600 \mathrm{~nm}}$ and expressed as a percentage of the growth in the minus drug (DMSO alone) control wells. (B and C) Growth of the $C$. albicans vps $21 \Delta / \Delta$ mutant and control strains was compared in the presence of various concentrations of the $\mathrm{Ca}^{2+}$ chelator EGTA without (B) or with $1 \mu \mathrm{g} / \mathrm{mL}$ of fluconazole (C). Growth was determined as above and is expressed as a percentage of the growth in the minus drug (DMSO) control wells. (D and E) The susceptibility of the $v p s 21 \Delta / \Delta$ mutant to the $\mathrm{Ca}^{2+}$ channel blocker nisoldipine (NIS) (D) and its effect on the mutant's tolerance to fluconazole (E) was evaluated. Growth was determined and expressed as above. ( $F$ and $\mathrm{G})$ The effect of the antiarrhythmic drug amiodarone (AMD) on the $v p s 21 \Delta / \Delta$ mutant's azole tolerance was tested. Growth was determined and expressed as above. The means \pm standard deviation of two biological replicates is shown in each panel. The means of each group were compared using a two-tailed $t$-test, ${ }^{*} P<0.05$. 
A.

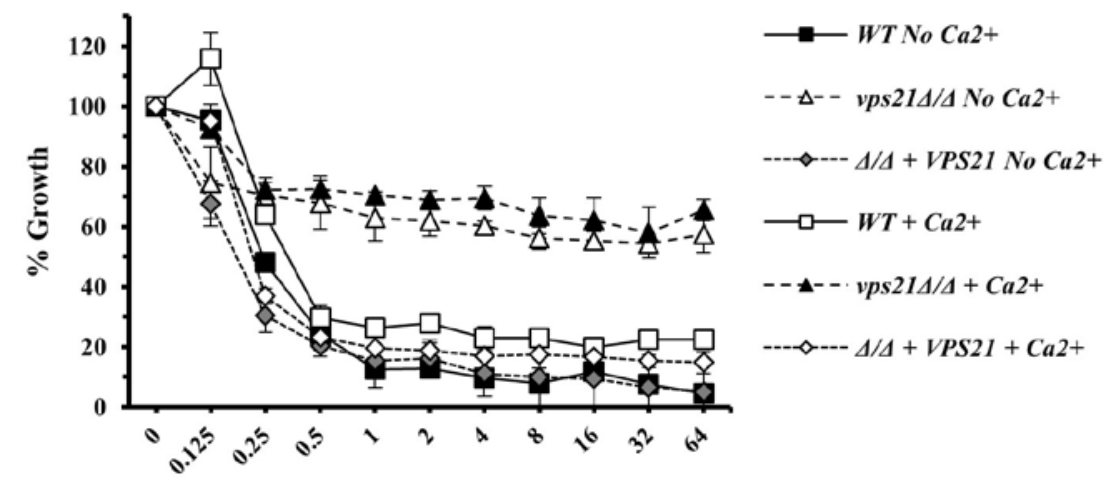

Fluconazole $[\mu \mathrm{g} / \mathrm{mL}]$

B.

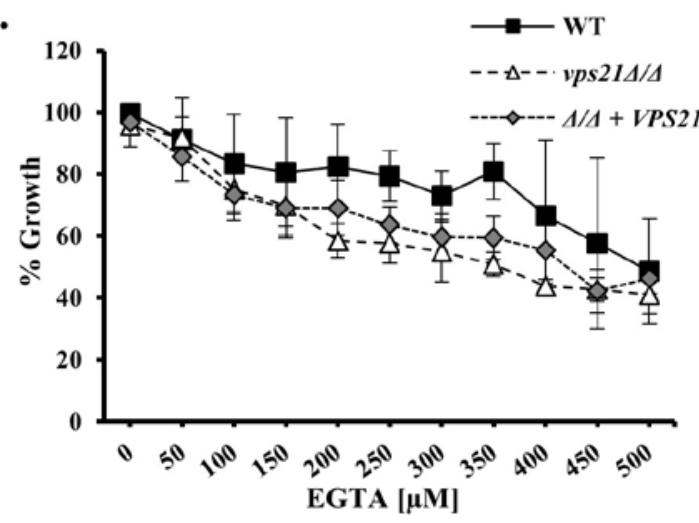

D.

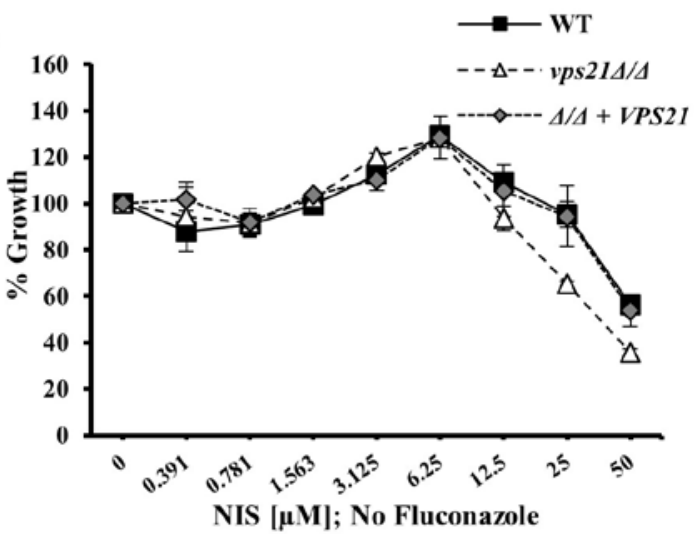

F.

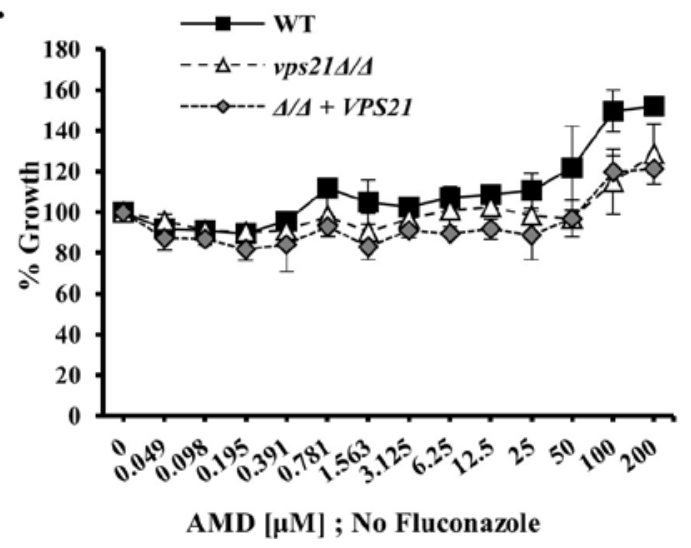

C.

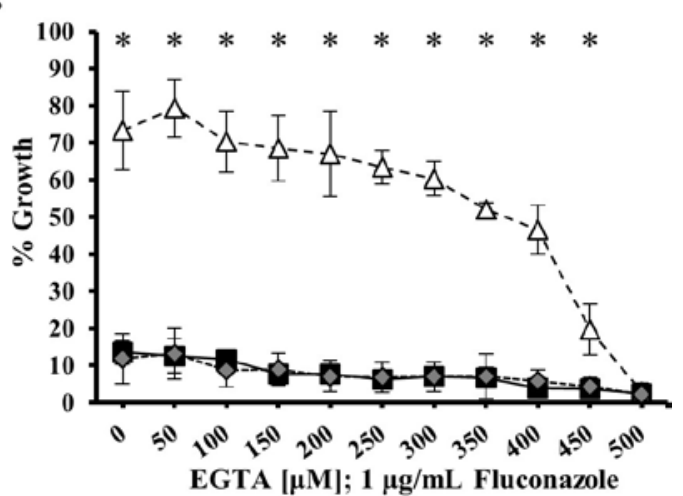

E.

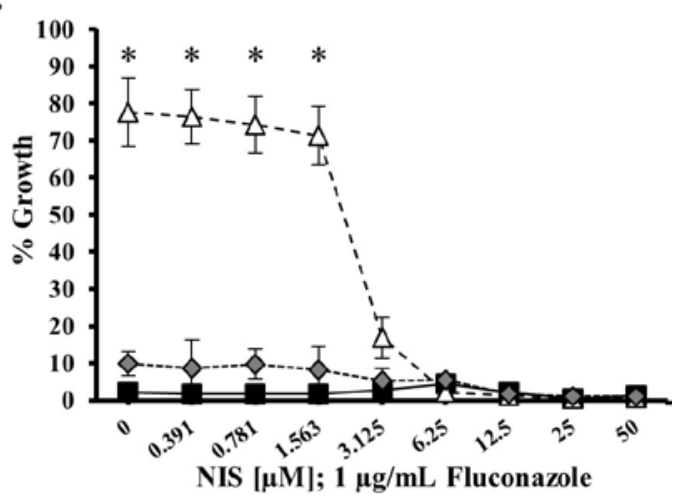

G.

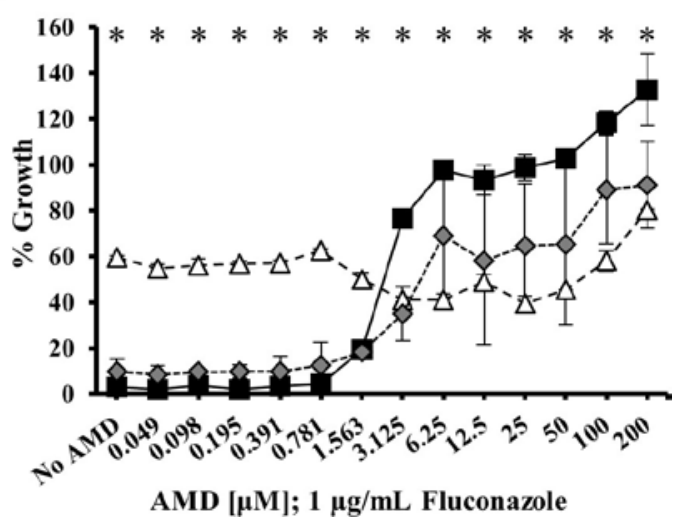


inhibition of this pathway renders the normally fungistatic azoles fungicidal $(147,150$, 241). Upon azole exposure, increased calcineurin activity enhances the transcription of a number of specific genes involved in plasma membrane maintenance and stress response, including RTA2 $(148,242)$. In order to evaluate the state of calcineurin signaling in the $v p s 21 \Delta / \Delta$ mutant, we compared the transcriptional activity of the calcineurin responsive gene RTA2 in mutant and control strains using a GFP $\gamma$ based reporter construct. RTA2 is a stress related gene that encodes a phospholipid translocase whose expression depends on the calcineurin-dependent transcription factor Crz1p $(151,243,244)$ and has been found to be upregulated in experimentally-induced azole resistant isolates (245). As expected, treatment with fluconazole enhanced GFP $\gamma$ expression from the RTA2 promoter in the WT control strain (Figure 4-6A). Furthermore, treatment with the calcineurin inhibitor cyclosporine A suppressed fluconazole induced expression of GFP $\gamma$ (Appendix B, Figure B-1), establishing the responsiveness of this reporter construct to calcineurin activity. Interestingly, treatment of the $v p s 21 \Delta / \Delta$ mutant with fluconazole induced a significantly larger response from the RTA2pr-GFP $\gamma$ reporter than was observed for the WT control strain, indicating an enhanced calcineurin dependent response in the mutant after azole exposure (Figure 4-6A).

In order to further define the role of calcineurin activity in the vps21 $1 / \Delta$ mutant's azole tolerance, we evaluated the impact of pharmacological inhibition of calcineurin signaling on the vps $21 \Delta / \Delta$ mutant and control strains after fluconazole exposure (Figure 4-6B-D). Inhibition of calcineurin signaling with either cyclosporine A (Figure 4-6C) or tacrolimus (FK506) (Appendix B, Figure B-2A-C), eliminated the $v p s 21 \Delta / \Delta$ mutant's azole tolerance. Collectively, these data indicate that the ability of the vps $21 \Delta / \Delta$ mutant to tolerate azole exposure is dependent upon calcineurin activity.

\section{Discussion}

In previous chapters we established that the azole antifungals cause severe disruption of the $C$. albicans vacuole (Chapter 2) and that trafficking through the late endosome/PVC mediated by the Rab GTPase Vps21p is a determining factor of $C$. albicans response to azole exposure. Our vps $21 \Delta / \Delta$ mutant was able to tolerate azole treatment and grow despite ergosterol depletion (Chapter 3), but unexpectedly, the mutant was more sensitive to exposure to other ergosterol biosynthesis inhibitors than the control strains. The growth of the mutant in the presence of azole antifungals resembles the 'trailing' growth phenomenon that is observed for some clinical isolates when treated with triazoles and was independent of established mechanisms of azole resistance. Our results reveal that the $C$. albicans vps $21 \Delta / \Delta$ mutant releases less cytoplasmic content than WT strains following fluconazole treatment at $35^{\circ} \mathrm{C}$, suggesting it sustains less membrane damage. In addition, we determined that the vps $21 \Delta / \Delta$ mutant has elevated levels of intracellular $\mathrm{Ca}^{2+}$ and that its growth in the presence of the azoles depends upon $\mathrm{Ca}^{2+}$ availability. This indicates that trafficking through the PVC mediated by Vps $21 \mathrm{p}$ is not only important in maintaining $\mathrm{Ca}^{2+}$ homeostasis in C. albicans, but that the $\mathrm{Ca}^{2+}$ homeostasis defects that arise from defective endosomal trafficking have substantial 
Figure 4-6. Inhibition of calcineurin signaling suppresses the $v p s 21 \Delta / \Delta$ mutant's azole tolerance.

(A) The RTA2pr-GFPy expression construct was introduced into $C$. albicans strain CAI4 (WT) and vps $21 \Delta / \Delta$ mutant, and the resulting strains were grown in $\mathrm{YNB} \pm 1 \mu \mathrm{g} / \mathrm{mL}$

fluconazole for 24 hours. GFP fluorescence was quantified by excitation at $488 \mathrm{~nm}$ and emission at $507 \mathrm{~nm}$, normalized for the cell density $\left(\mathrm{OD}_{600 \mathrm{~nm}}\right)$ of each strain and expressed as fold fluorescence of the DMSO control. The means \pm standard deviations of four biological replicates are shown. The means of each group were compared using a two-tailed $t$-test: $* P<0.0004$. (B-D) The growth of the $v p s 21 \Delta / \Delta$ mutant, WT (YJB6284) and reconstituted strain in the presence of both the calcineurin activity inhibitor cyclosporine A (CsA) and fluconazole was compared using a checkerboard assay. The growth of WT (B), vps $21 \Delta / \Delta$ mutant (C), and isogenic control strain (D) was determined as $\mathrm{OD}_{600 \mathrm{~nm}}$ and expressed as a percentage of the growth in the minus drug (DMSO) control wells. Data from a representative experiment is shown, but similar results were obtained in a second repeat experiment. 
A.

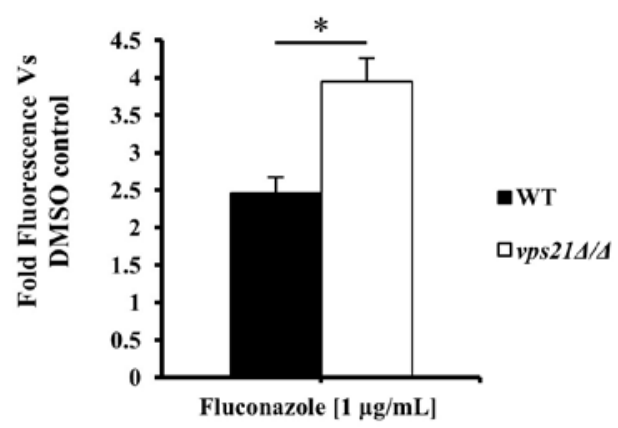

B.

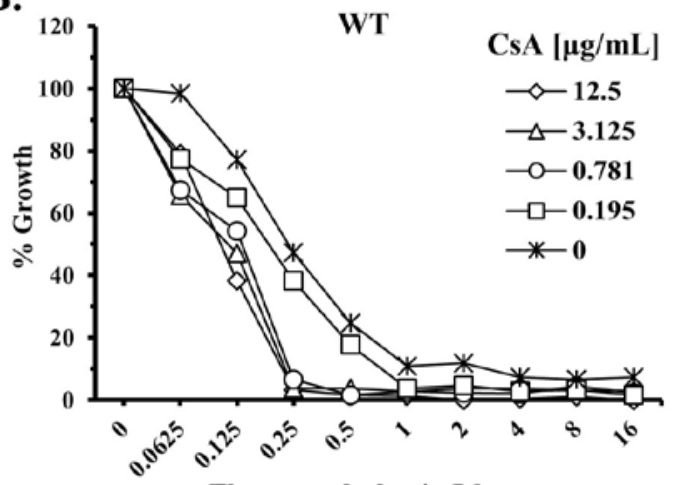

Fluconazole $[\mu \mathrm{g} / \mathrm{mL}]$
C.

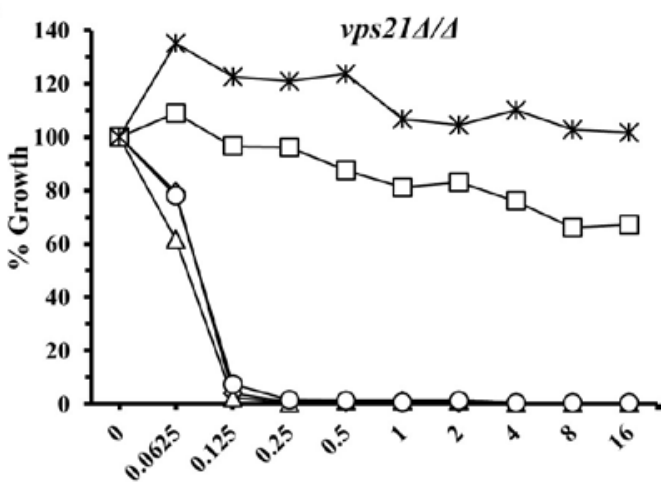

Fluconazole $[\mu \mathrm{g} / \mathrm{mL}]$

D.

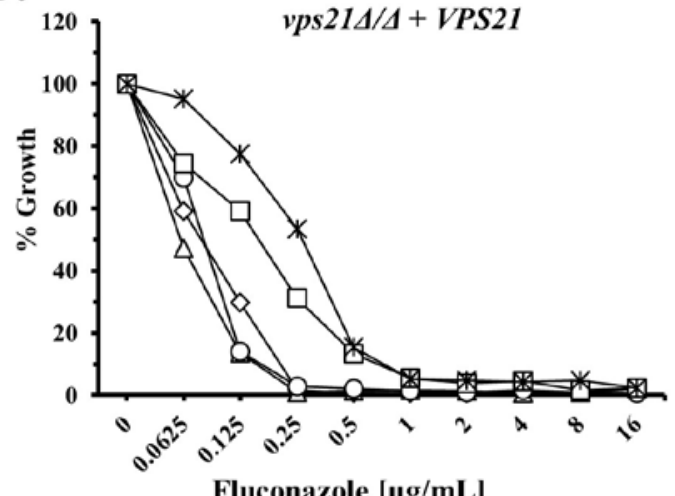

Fluconazole $[\mu \mathrm{g} / \mathrm{mL}]$ 
consequences in the response of $C$. albicans against the most important class of antifungals in clinical use.

We also determined that the ability of the $\operatorname{vps} 21 \Delta / \Delta$ mutant to tolerate azole exposure is dependent upon signaling through the $\mathrm{Ca}^{2+}$-responsive phosphatase calcineurin. In $C$. albicans and other fungi, the role for $\mathrm{Ca}^{2+}$-dependent calcineurin signaling in the cellular response to azole antifungals has been widely described (147). Upregulation of calcineurin signaling enhances the survival of the fungal cell by expressing a multitude of genes involved in the response against membrane stressors, including azole antifungals $(148,242)$. Importantly, genetic or pharmacological inhibition of calcineurin signaling renders the normally static azoles into cidals $(149,150)$. As suggested by our reporter assay in Figure 4-6A, abnormal $\mathrm{Ca}^{2+}$ homeostasis could potentially increase the basal level of calcineurin activity and/or affect the magnitude or duration of calcineurin signaling in the vps $21 \Delta / \Delta$ mutant upon azole exposure. This in turn may increase the chances of the mutant's survival by, for example, minimizing the deleterious effects of azole treatment upon plasma membrane integrity. Consequently, it was not surprising that inhibition of calcineurin signaling with CsA or FK506 rendered the $v p s 21 \Delta / \Delta$ mutant susceptible to fluconazole. Interestingly, previous studies showed that membrane trafficking mutants of $C$. albicans defective in endosome-to-Golgi retrograde transport have constitutive activation of calcineurin signaling and enhanced expression of genes involved in stress responses (222). However, these mutants were hypersensitive to azole treatment. Collectively, these findings suggest that the specific PVC trafficking step affected is a crucial determinant of the physiological impact of the azoles upon the fungal cell. 


\section{CHAPTER 5. THE CANDIDA ALBICANS MUTANT LACKING THE RAB GTPASE VPS21P IS SUSCEPTIBLE TO AZOLE TREATMENT IN VIVO}

\section{Introduction}

In previous chapters we showed that the C. albicans vps $21 \Delta / \Delta$ mutant defective in trafficking through the PVC is tolerant to azole exposure under standard susceptibility testing conditions. This tolerance phenotype resembled the "trailing growth" phenomenon that is observed in a small percentage of clinical isolates of C. albicans, whereby an isolate that appears susceptible to azole treatment at 24 hours, is capable of continued growth and seemingly resistant at the 48 hour time-point $(143,144)$. We also determined that the tolerance of the vps $21 \Delta / \Delta$ mutant to azole antifungals is dependent upon $\mathrm{Ca}^{2+}$ and calcineurin signaling.

The contribution of several mechanisms of azole resistance, such as overexpression of the target enzyme Erg11p and/or efflux pumps Cdr1p and Mdr1p, to the trailing growth phenomenon has been investigated (123). However, their role as causative of this form of azole tolerance has not been conclusive. Although "trailing isolates" are capable of tolerating azole exposure in vitro, they appear to be susceptible when exposed to azoles in mouse models of disseminated C. albicans infection (143, 144). However, the possible interactions between azole resistance mechanisms and the trailing growth phenomenon have not been explored, in part due to the fact that the molecular mechanisms responsible for the trailing growth phenomenon have not been completely defined. The objective of the studies described in this chapter was to define whether defective endosomal trafficking synergizes or antagonizes with known azole resistance mechanisms. In addition, we sought to determine if the azole tolerance of the $v p s 21 \Delta / \Delta$ mutant affects the in vivo antifungal efficacy of the azoles.

\section{Materials and Methods}

\section{Growth and media conditions}

C. albicans was routinely grown in YPD medium (1\% yeast extract, $2 \%$ peptone, $2 \%$ dextrose $)$ at $30^{\circ} \mathrm{C}$, supplemented with uridine $(50 \mu \mathrm{g} / \mathrm{mL})$ when necessary. Transformant selection was carried out on minimal YNB medium $(6.75 \mathrm{~g} / \mathrm{L}$ yeast nitrogen base without amino acids, $2 \%$ dextrose, $2 \%$ Bacto agar), supplemented with the appropriate auxotrophic requirements as described for $S$. cerevisiae (185) or $50 \mu \mathrm{g} / \mathrm{mL}$ uridine. 


\section{Plasmid construction}

All oligonucleotides used in this study are listed in Table 5-1. Plasmid pKE1 was previously described (187). Plasmid pKE4 was constructed by replacing the ACT1 promoter from $\mathrm{pKE} 1$ with the $T E F 1$ promoter which was amplified with primers TEF1pF-KpnI and TEF1pR-SalI from SC5314 genomic DNA (gDNA), and cloned using KpnI and SalI restriction sites of pKE1.

The ERG11 ORF was amplified from SC5314 gDNA with HiFi Platinum Taq (Invitrogen) and primer set ERG11ORFF + ERG11ORFR, and cloned between the SalI and $M l u I$ restriction sites of pKE4 to produce plasmid pKE4ERG11.

Plasmid pKE4CDR1 was produced by amplifying the CDR1 ORF from SC5314 gDNA with HiFi Platinum Taq (Invitrogen) and primer pair CDR1ORFF-SalI +

CDR1ORFR-MluI, and then cloned between the SalI and MluI restriction sites of pKE4.

The CDR2 ORF was amplified from SC5314 gDNA with HiFi Platinum Taq (Invitrogen) and primer pair CDR2ORFF-SalI + CDR2ORFR-MluI, and cloned between the SalI and MluI restriction sites of pKE4 to produce plasmid pKE4CDR2.

For construction of the plasmid pKE4MDR1, the MDR1 ORF was amplified with HiFi Platinum Taq (Invitrogen) and primer pair MDR1ORFF-EagI + MDR1ORFR-MluI from SC5314 gDNA, and cloned between the EagI and MluI restriction sites of pKE4 to produce plasmid pKE4MDR1.

\section{C. albicans strain construction}

Strains used in this study are listed in Table 5-2. Transformation of C. albicans with DNA constructs was performed using the lithium acetate method (217). Gene deletion strains were constructed by the PCR-based approach described by Wilson et al. (190) using the $u r a 3 \Delta / \Delta$ his $1 \Delta / \Delta \arg 4 \Delta / \Delta$ strain BWP17 (kindly provided by Dr. Aaron Mitchell, Carnegie Mellon University). Strain CAI4 was kindly provided by Dr. William Fonzi (Georgetown University). Strain SC5314 has been previously described (216). Control strain YJB6284 (215) was kindly provided by Dr. Judith Berman (Tel Aviv University). The azole resistant strain AR46606 was kindly provided by Dr. Mairi C. Noverr (Louisiana State University Health Sciences Center).

The vps $21 \Delta / \Delta$ and $v p s 21 \Delta / \Delta u r a 3 \Delta / \Delta$ were constructed in previous studies (51). CAI4 and the vps $21 \Delta / \Delta u r a 3 \Delta / \Delta$ were transformed with pKE4, pKE4ERG11, pKE4CDR1, pKE4CDR2, pKE4MDR1. Correct integration of the above plasmids fully restores the URA3 and adjacent IROI loci, and this was confirmed by PCR analysis using primer pair LUXINTDETF + LUXINTDETR which yields a 2199 bp product. 
Table 5-1. List of oligonucleotides used in the study described in Chapter 5.

\begin{tabular}{ll}
\hline \multicolumn{1}{c}{ Primer } & \multicolumn{1}{c}{ Sequence (5'-3') } \\
\hline CDR1ORFF-SalI & TCAGTCGACATGTCAGATTCTAAGATGTCG \\
CDR1ORFR-MluI & TCAACGCGTGACGTTTGAGATACCACCATGTC \\
CDR2ORFF-SalI & TCAGTCGACATGAGTACTGCAAACACGTCT \\
CDR2ORFR-MluI & TCAACGCGTTTGTAAAATAAGACCCCATCC \\
ERG11ORFF & TCAGTCGACATGGCTATTGTTGAAACTGTC \\
ERG11ORFR & TCAACGCGTTGAATCGAAAGAAAGTTGCCG \\
LUXINTDETF & CTGACCTTTAGTCTTTCCTC \\
LUXINTDETR & CAGTAGTACTTGTTGTTGTATCG \\
MDR1ORFF-EagI & TCACGGCCGATGCATTACAGATTTTGAGAG \\
MDR1ORFR-MluI & TCAACGCGTATAGGAAAACAATGACACCTC \\
TEF1pF-KpnI & TCAGGTACCTGCAAATCTGTTTGCTGATGG \\
TEF1pR-SaII & TCAGTCGACGATTGATTATGACTATAATGTG \\
\hline
\end{tabular}


Table 5-2. List of strains used in the study described in Chapter 5.

\begin{tabular}{|c|c|c|}
\hline Strain & Relevant Genotype & Reference \\
\hline SC5314 & Wild-type/clinical isolate & (229) \\
\hline CAI4 & $u r a 3 \Delta / \Delta$ & $(193)$ \\
\hline BWP17 & ura $3 \Delta / \Delta$ his $1 \Delta / \Delta \arg 4 \Delta / \Delta$ & $(190)$ \\
\hline YJB6284 & ura3 $\Delta / \Delta: U R A 3$ his $1 \Delta / \Delta: H I S 1 \arg 4 \Delta / \Delta: A R G 4$ & $(215)$ \\
\hline AR46606 & Azole resistant clinical isolate & Noverr Lab \\
\hline$v p s 21 \Delta / \Delta$ & ura $3 \Delta / \Delta: U R A 3$ his $1 \Delta / \Delta \arg 4 \Delta / \Delta$ vps $21 \Delta: H I S 1 / v p s 21 \Delta: A R G 4$ & $(51)$ \\
\hline $\operatorname{vps} 21 \Delta / \Delta+\operatorname{VPS} 21$ & 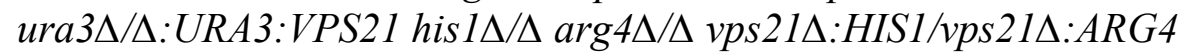 & $(51)$ \\
\hline CAI4 + TEF1prERG11 & 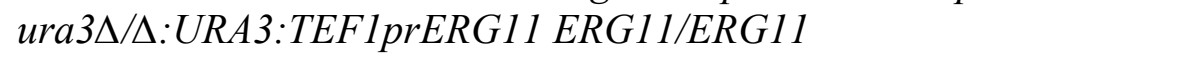 & This study \\
\hline $\operatorname{vps} 21 \Delta / \Delta+T E F 1 p r E R G 11$ & $\begin{array}{l}\text { ura3 } \Delta / \Delta: U R A 3: T E F 1 p r E R G 11 \text { his } 1 \Delta / \Delta \arg 4 \Delta / \Delta \\
\text { vps } 21 \Delta: H I S 1 / v p s 21 \Delta: A R G 4 \text { ERG11/ERG11 }\end{array}$ & This study \\
\hline $\mathrm{CAI} 4+T E F 1 p r C D R 1$ & ura3A/A:URA3:TEF1prCDR1 CDR1/CDR1 & This study \\
\hline$v p s 21 \Delta / \Delta+T E F 1 p r C D R 1$ & $\begin{array}{l}\text { ura3 } \Delta / \Delta: U R A 3: T E F 1 p r C D R 1 \text { his } 1 \Delta / \Delta \arg 4 \Delta / \Delta \\
\text { vps } 21 \Delta: H I S 1 / v p s 21 \Delta: A R G 4 C D R 1 / C D R 1\end{array}$ & This study \\
\hline $\mathrm{CAI} 4+T E F 1 p r C D R 2$ & ura3A/A:URA3:TEF1prCDR2 CDR2/CDR2 & This study \\
\hline$v p s 21 \Delta / \Delta+T E F 1 p r C D R 2$ & $\begin{array}{l}\text { ura3 } \Delta / \Delta: U R A 3: T E F 1 p r C D R 2 \text { his } 1 \Delta / \Delta \arg 4 \Delta / \Delta \\
\text { vps } 21 \Delta: H I S 1 / v p s 21 \Delta: A R G 4 C D R 2 / C D R 2\end{array}$ & This study \\
\hline $\begin{array}{l}\text { CAI4 + TEF1prMDR1 } \\
\text { vps } 21 \Delta / \Delta+\text { TEF1prMDR1 }\end{array}$ & 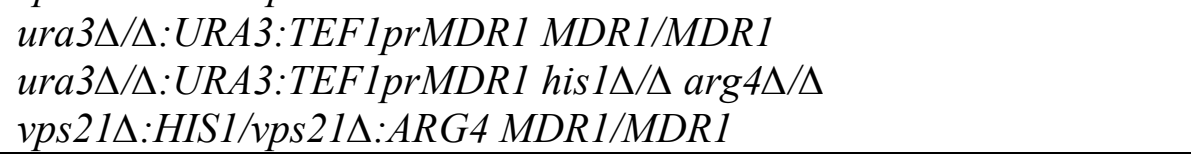 & $\begin{array}{l}\text { This study } \\
\text { This study }\end{array}$ \\
\hline
\end{tabular}




\section{Antifungal susceptibility testing}

Antifungal susceptibility testing of all the strains was performed using the broth micro-dilution method described in the CLSI document M27-A3 (218) in a 96-well plate format. Fluconazole was diluted in DMSO in 2-fold dilutions at 200 times the final concentration. RPMI 1640 medium (Sigma-Aldrich) was prepared according to the CLSI document (218), and the medium was buffered with morpholinepropanesulfonic acid (MOPS) and $\mathrm{pH}$ adjusted to 7.0 using $\mathrm{NaOH}$. Plates were incubated without shaking at $35^{\circ} \mathrm{C}$ for 24 or 48 hours unless otherwise stated. The content of each well was carefully resuspended by pipetting up and down before $\mathrm{OD}_{600 \mathrm{~nm}}$ was measured using a Cytation ${ }^{\mathrm{TM}}$ 5 plate reader (BioTek Instruments, Inc.).

\section{Mouse model of vaginal candidiasis and treatment of the vps21 $\Delta / \Delta$ mutant with fluconazole in vivo}

The murine model of Candida vaginitis has been reported extensively in the literature and was performed as described previously (246). The animals used in this study were housed in AAALAC-approved facilities located at the Louisiana State University Health Sciences Center (LSUHSC) in the School of Dentistry. The LSUHSC Animal Care and Use Committee approved all animals and protocols. Mice were given standard rodent chow and water ad libitum. Mice were monitored daily for signs of distress, including noticeable weight loss and lethargy. C57BL/6 mice were purchased from Jackson Laboratories and housed in isolator cages mounted onto ventilated racks. Mice were administered $0.1 \mathrm{mg}$ of estrogen ( $\beta$-estradiol 17-valerate; Sigma) dissolved in $0.1 \mathrm{~mL}$ sesame oil subcutaneously 72 hours prior to inoculation with $C$. albicans. Estrogen injections were administered weekly thereafter. Stationary-phase cultures of $C$. albicans isolates were washed three times in sterile, endotoxin-free phosphate-buffered saline (PBS) and resuspended in a $0.2 \mathrm{X}$ volume of PBS. Cell suspensions were diluted, counted on a Neubauer hemocytometer, and adjusted to $2.5 \times 10^{8} \mathrm{CFU} \mathrm{mL}^{-1}$ in sterile PBS. Estrogen-treated mice were intravaginally inoculated with $20 \mu \mathrm{L}$ of the standardized cell suspension, generating an inoculum size of $5 \times 10^{6}$ blastoconidia. Animals were infected with the following strains: SC5314 (WT), the vps $21 \Delta / \Delta$ mutant, the complemented strain (vps $21 \Delta / \Delta+V P S 21)$, and the azole resistant vaginal isolate AR46606. For fluconazole treatment, animals infected with each strain were divided into two groups of five mice each. Every group was administered fluconazole at $25 \mathrm{mg} / \mathrm{kg}$ or vehicle at day 4 post inoculation by oral gavage. Vaginal lavage was carried out at day 7 and 10 post inoculation to determine colony forming units (CFUs) as a measure of infection/clearance. 


\section{Results}

The $C$. albicans vps $21 \Delta / \Delta$ mutant is susceptible to azole treatment in a mouse model of vaginal candidiasis

Although trailing isolates are tolerant to azole treatment in vitro, in vivo studies conducted in mouse models of disseminated candidiasis suggest that such isolates are susceptible to azole treatment in vivo $(143,144)$. In order to determine whether the $v p s 21 \Delta / \Delta$ mutant is susceptible or tolerant to azole treatment in vivo, and to establish the clinical significance of endosomal trafficking with respect to azole tolerance, we tested the vps $21 \Delta / \Delta$ mutant's response to fluconazole treatment in a mouse model of vaginal candidiasis. Mice were treated with estrogen in order to establish and maintain $C$. albicans infection. After 4 days post-infection, animals were treated with $25 \mu \mathrm{g} / \mathrm{mL}$ of fluconazole or vehicle, and clearance was determined as CFU from vaginal lavage at day 7 and 10.

The vps $21 \Delta / \Delta$ mutant was able to colonize the mouse vagina at levels similar to the control strains, as indicated by the CFUs of untreated animals at day 7 and 10 post infection (Figure 5-1A and B). Treatment with fluconazole reduced the CFUs of the vps $21 \Delta / \Delta$ mutant at day 7 and to a lesser degree at day 10 post infection (Figure 5-1A), to a similar extent to that observed in the susceptible VPS21+ control strains. This suggests that the $\operatorname{vps} 21 \Delta / \Delta$ mutant is susceptible to azole treatment in vivo, like the susceptible control strains. Thus, the vps $21 \Delta / \Delta$ mutant's azole tolerance in vitro does not seem to translate into reduced susceptibility in vivo or increased fungal persistence upon azole treatment in the mouse vagina.

\section{Overexpression of ERG11 antagonizes azole tolerance in the $C$. albicans vps $21 \Delta / \Delta$ mutant}

The need to explore the role of trailing growth in the development of azole resistance has been suggested previously (123). Azole resistance mechanisms such as overexpression of ERG1 1 as well as $C D R 1, C D R 2$ and $M D R 1$, and amino acid substitutions in Erg $11 \mathrm{p}$ have been described in a multitude of azole resistant clinical isolates of $C$. albicans (81). However, a combination of these mechanisms is usually required to confer high levels of azole resistance (125). Moreover, there are a number of resistant clinical isolates in which no known mechanisms of azole resistance have been identified. Therefore we aimed to determine whether deficient endosomal trafficking may synergize or antagonize with known azole resistance mechanisms in $C$. albicans. To address this, we overexpressed the genes encoding for the target enzyme ERG11, and the efflux pumps $C D R 1, C D R 2$, and $M D R 1$ from the $T E F 1$ promoter in the vps $21 \Delta / \Delta$ mutant and tested the susceptibility of the "overexpressor" strains to fluconazole.

Overexpression of the genes encoding the efflux pumps did not significantly affect the tolerance to fluconazole of the $v p s 21 \Delta / \Delta$ (Figure 5-2A-C), indicating that 
A.

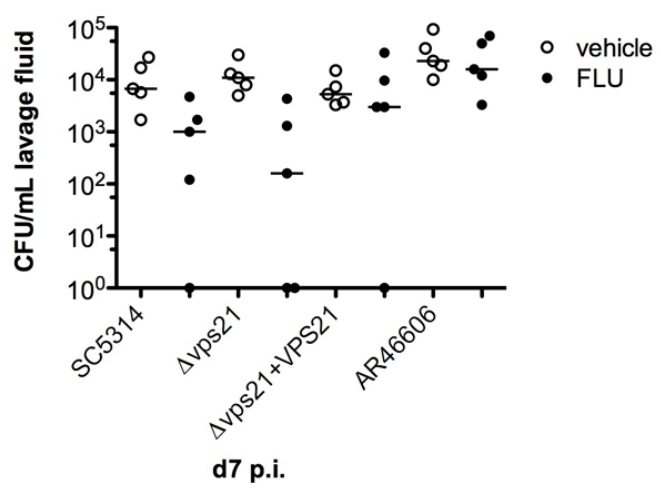

B.

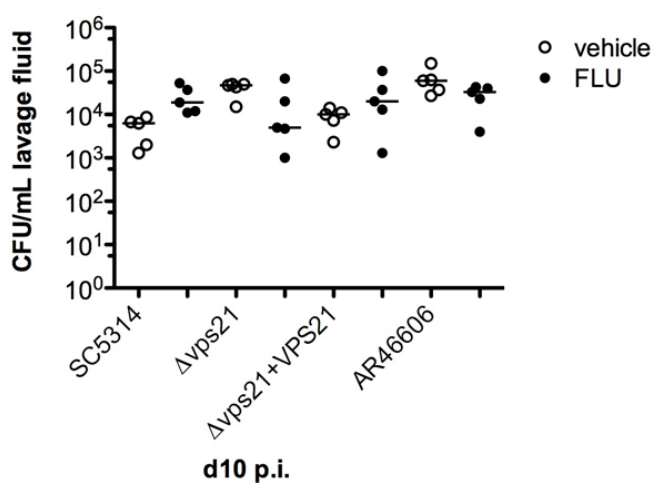

Figure 5-1. The $C$. albicans vps $21 \Delta / \Delta$ mutant is susceptible to azole treatment in a mouse model of vaginal candidiasis.

The susceptibility to fluconazole of the WT (SC5314), vps $21 \Delta / \Delta$ mutant, complemented control strain and the azole resistant clinical isolate AR46606 was compared in a mouse model of vaginal candidiasis. For each strain tested, groups of four animals were treated with $25 \mu \mathrm{g} / \mathrm{mL}$ of fluconazole or vehicle by oral gavage at 4 days post-infection (p.i.). Clearance/infection was determined as colony forming units (CFU) from vaginal lavage carried out at day (A) 7 and (B) 10 p.i. 
Figure 5-2. Overexpression of efflux pumps does not synergize or antagonize azole tolerance in the $C$. albicans vps $21 \Delta / \Delta$ mutant.

The genes encoding for the efflux pumps $M d r 1 p(A), C d r 1 p(B)$, and $C d r 2 p$ were expressed from the TEF1 promoter in CAI4 (WT) and vps $21 \Delta / \triangle$ mutant. The susceptibility to fluconazole of the overexpressing and control strains was compared using the standard CLSI broth microdilution protocol. Following 48 hours incubation, growth was measured as $\mathrm{OD}_{600 \mathrm{~nm}}$ and expressed as a percentage of the growth in the minus drug (DMSO alone) control wells. The means \pm standard deviations of two biological replicates are shown. 
A.

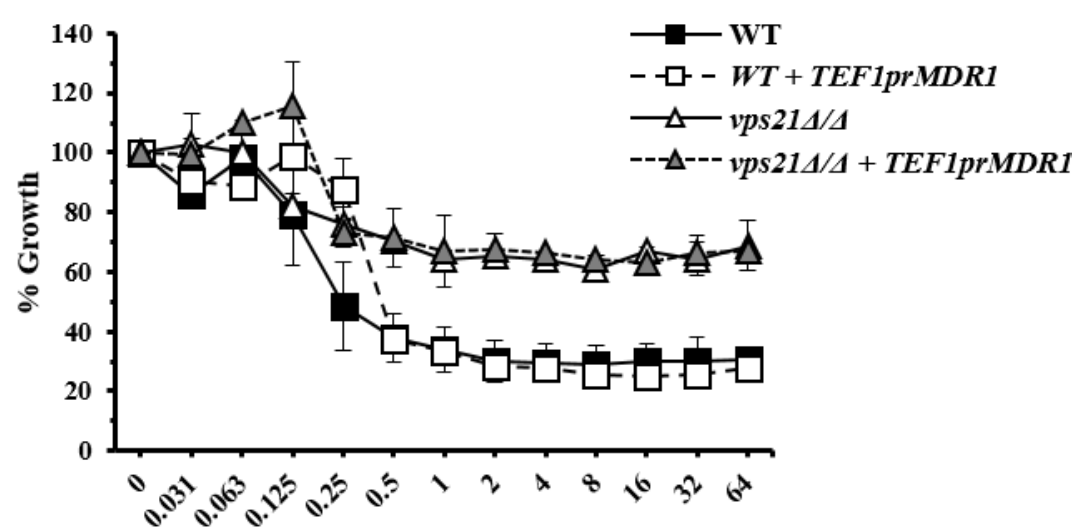

Fluconazole $[\mu \mathrm{g} / \mathrm{mL}]$

B.

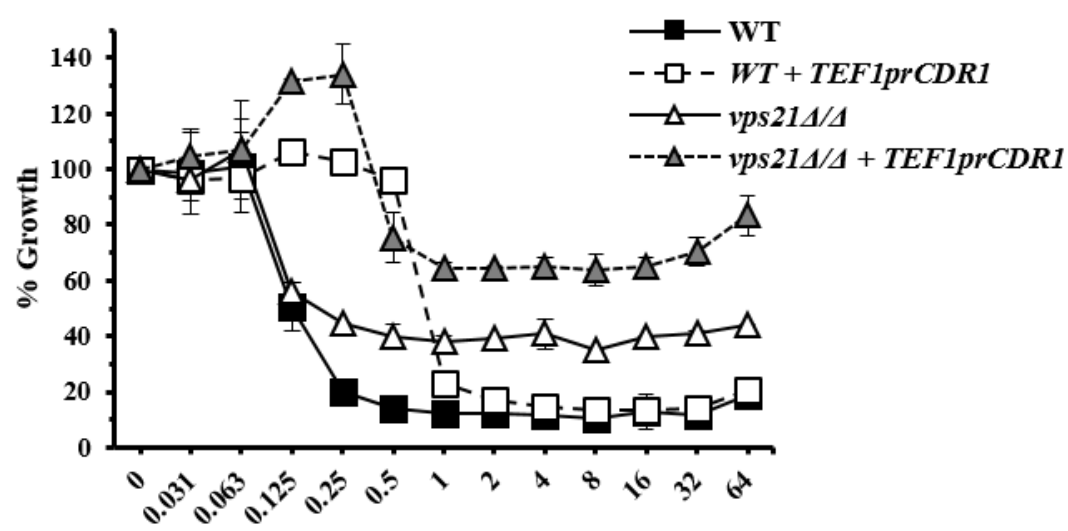

Fluconazole $[\mu \mathrm{g} / \mathrm{mL}]$

C.

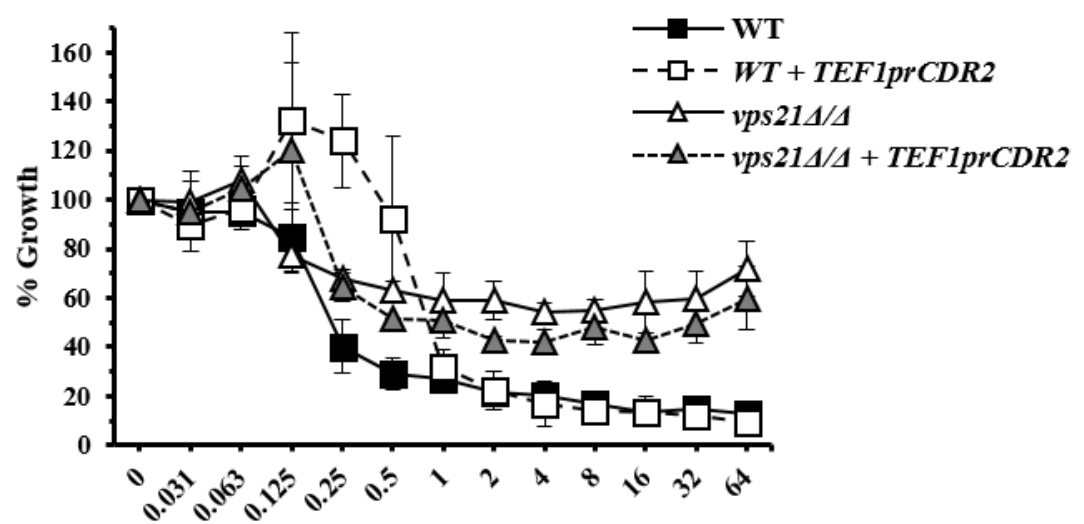

Fluconazole $[\mu \mathrm{g} / \mathrm{mL}]$ 
overexpression of efflux pumps does not synergize or antagonize with endosomal trafficking. Importantly, overexpression of $C D R 1$ in the $v p s 21 \Delta / \Delta$ mutant negatively affected the growth of the resulting strain in the control wells (no drug), but not in the presence of fluconazole as determined by $\mathrm{OD}_{600 \mathrm{~nm}}$ (data not shown). Therefore it seems apparent that in the presence of fluconazole, the percentage growth of the vps $21 \Delta / \Delta$ mutant overexpressing $C D R l$ is elevated with respect to the $v p s 21 \Delta / \Delta$ control strain, although the $\mathrm{OD}_{600 \mathrm{~nm}}$ are similar (data not shown).

Interestingly, overexpression of ERG11 significantly reduced the fluconazole tolerance at the 48 hour time point, despite increasing the MIC of the mutant by 24 hours (Figure 5-3A and B). These suggests that overexpression of ERG11 antagonizes azole tolerance dependent on altered endosomal trafficking.

\section{Discussion}

Thus far we have determined that the $C$. albicans vps $21 \Delta / \Delta$ mutant tolerates azole exposure, and this ability depends upon both $\mathrm{Ca}^{2+}$ and calcineurin signaling. Furthermore, we determined that the pattern of growth of the mutant in the presence of azoles resembled that of "trailing" isolates of $C$. albicans. In order to determine the clinical relevance of these findings, we studied possible interactions between azole tolerance as a result of loss of Vps21p-mediated trafficking through the PVC and known azole resistance mechanisms. No synergism or antagonism seems to occur between loss of Vps21p and overexpression of genes encoding for the efflux pumps Cdr1p, Cdr2p and Mdr1p. However, we found an antagonistic relationship between deletion of VPS 21 and overexpression of the gene encoding the azoles target enzyme Erg11p. Overexpression of ERG11 increased the MIC in both vps $21 \Delta / \Delta$ mutant and wild-type after 24 hours incubation, but it reduced the tolerance of the mutant at the 48 hour time point. This is interesting, since in Chapter 3 we showed that the tolerance phenotype of the $v p s 21 \Delta / \Delta$ mutant was dependent upon Erg11p inhibition, reflecting a complex interaction between the expression of Erg11p and disruption of endosomal trafficking mediated by Vps21p.

We also tested the susceptibility of the vps $21 \Delta / \Delta$ mutant to azole treatment in vivo using a mouse model of vaginal candidiasis. We selected this model based on several considerations. First, the vps $21 \Delta / \Delta$ mutant has been shown to have reduced virulence in a mouse model of disseminated candidiasis, which can complicate the interpretation of results if the susceptibility to fluconazole is tested in this model. Secondly, the mouse vagina has a temperature of around $35^{\circ} \mathrm{C}$ and a neutral $\mathrm{pH}$, similar to those used in the CLSI protocol for susceptibility testing. Several studies performed to date have suggested that the trailing growth phenotype is usually associated with in vivo susceptibility to azole therapy $(143,144)$. In this context, we determined that our vps $21 \Delta / \Delta$ mutant was susceptible to azole treatment in vivo in a mouse model of vaginal candidiasis. These susceptibility results are analogous to those obtained for clinical trailing isolates in mouse models of disseminated candidiasis $(143,144)$. However, whether or not endosomal trafficking defects contribute to the trailing growth phenomenon in clinical isolates is a 
A.

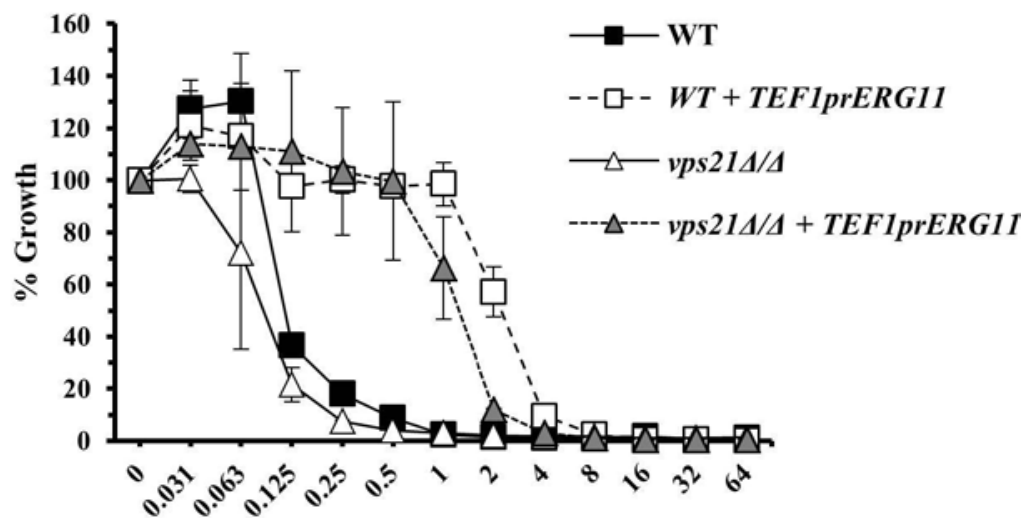

Fluconazole $[\mu \mathrm{g} / \mathrm{mL}] ; 24 \mathrm{~h}$

B.

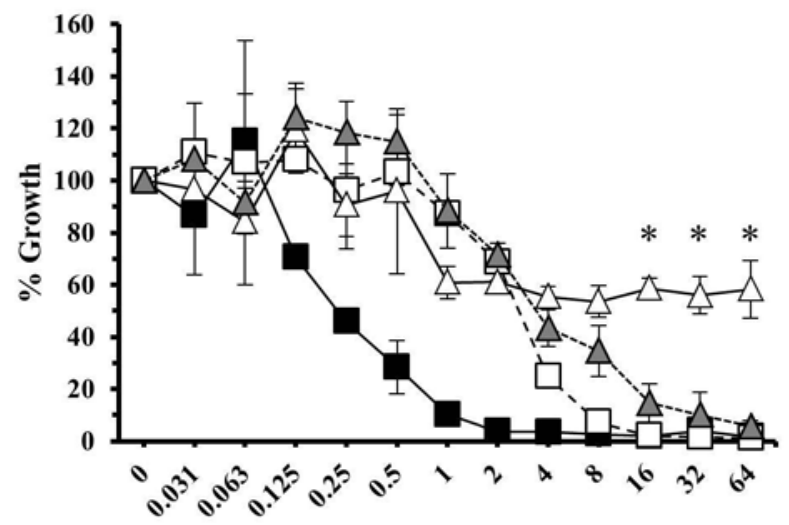

Fluconazole $[\mu \mathrm{g} / \mathrm{mL}] ; 48 \mathrm{~h}$

Figure 5-3. Overexpression of ERG11 reduces azole tolerance in the C. albicans vps21 $\Delta / \Delta$ mutant.

The ERG11 ORF was expressed from the TEF1 promoter in CAI4 (WT) and $v p s 21 \Delta / \Delta$ mutant. The susceptibility to fluconazole of the $E R G 11$-overexpressing and control strains was evaluated using the standard CLSI broth microdilution protocol. Following 24 (A) and 48 hours (B) incubation, growth was measured as $\mathrm{OD}_{600 \mathrm{~nm}}$ and expressed as a percentage of the growth in the minus drug (DMSO alone) control wells. The mean \pm standard deviations of two biological replicates are shown. The means of each group were compared using a two-tailed $t$-test. $* P<0.05$, between the $v p s 21 \Delta / \Delta$ mutant and vps $21 \Delta / \Delta+T E F 1$ prERG11 strain. 
matter that has not been explored to date.

Collectively, these findings highlight the importance of Vps21p-mediated trafficking in determining the outcome of azole treatment in C. albicans both in vitro and in vivo. 


\section{CHAPTER 6. DISCUSSION $\dagger$}

\section{Azole Antifungals and Vacuolar Integrity}

The fungal lipid ergosterol is a major membrane component that plays key roles in supporting plasma membrane function and integrity $(79,80)$. It has been implicated in processes that include endocytosis, homotypic vacuole fusion, pheromone signaling and the function of several proteins $(81,82,169,170)$. The importance of ergosterol is such, that a number of antifungal drugs target its biosynthesis. The most important class of antifungals currently in clinical use is the azoles. They prevent the synthesis of ergosterol by inhibiting the enzyme lanosterol 14-demethylase (Erg11p). In addition, inhibition of Erg $11 \mathrm{p}$ leads to the accumulation of methylated sterols that ultimately insert in the plasma membrane and cause instability and loss of function that result in growth inhibition (92). Loss of plasma membrane function due to both the accumulation of toxic sterol species and ergosterol depletion in the plasma membrane have been widely acknowledged as the main antifungal mechanisms of azole antifungals (196). However, other intracellular membranes contain ergosterol, although in relatively smaller quantities (173). Therefore the integrity and function of intracellular membranes can be compromised due to azole treatment and inhibition of ergosterol biosynthesis. This opens the question of whether the antifungal activity of the azoles is only due to loss of plasma membrane integrity or if it is also associated with the dysfunction of intracellular membranes.

The vacuole is a membrane-bound compartment whose importance in stress responses and pathogenesis has been well defined in fungi $(156,166)$. In this work, we showed that treatment with azole antifungals severely compromise $C$. albicans vacuole integrity, and that this phenomenon is a result of Erg11p inhibition and not an off-target effect of azole treatment. Furthermore, vacuole disruption was shown to occur as an early event following drug exposure, before significant growth inhibition was observed. These findings support the notion that azole antifungals not only disrupt plasma membrane integrity but other intracellular membrane compartments are also affected. In addition to supporting C. albicans resistance to a multitude of different stresses, the vacuole has been shown to support hyphae formation and the ability of $C$. albicans to survive and cause lethality in vivo. C. albicans mutants with severe defects in vacuolar function have reduced growth rates, and are susceptible to multiple stresses, defective in filamentous growth and unable to cause lethality in a mouse model of disseminated candidiasis (49, $51,166)$. Thus it is possible that the impairment of vacuolar function as a result of azole exposure contributes to the antifungal activity of the azoles, in addition to the

$\uparrow$ Modified by permission of American Society for Microbiology. Luna-Tapia A, Tournu H, Peters TL, Palmer GE. 2016. Endosomal trafficking defects can induce calcium dependent azole tolerance in Candida albicans. Antimicrob Agents Chemother 60:000 000. doi:10.1128/AAC.01034-16. 
consequences on plasma membrane integrity. In this regard, recent work suggested that loss of ergosterol upon azole treatment affects the function of the V-ATPase responsible for vacuolar acidification, which is necessary for the function of hydrolytic enzymes and homotypic vacuole fusion (79). The function of a number of other vacuolar membrane proteins may also be affected by azole treatment, including amino acid exchangers, transporters, ion pumps and channels, among others.

Whether vacuole disruption following azole treatment is mediated by ergosterol depletion, by the accumulation of toxic sterol species in the membrane or a combination of both factors is less clear. Several methylated sterol species accumulate in the plasma membrane as a consequence of Erg $11 \mathrm{p}$ inhibition and results in increased permeability, defective membrane fluidity, and ultimately, growth inhibition (141). Among those, $14 \alpha-$ methylergosta-8,24(28)-dien- 3 $\beta, 6 \alpha$-diol amasses in large quantities in the plasma membrane in cells exposed to azoles and it is thought to play a major role in the mechanism of action of these antifungals (141). This sterol is a metabolic product of Erg3p after inhibition of ergosterol biosynthesis at the Erg11p step $(81,141)$.

Interestingly, deletion of $E R G 3$ ameliorated the impact of azole treatment on the integrity of the C. albicans vacuole, suggesting that the activity of Erg3p and presumably the production of toxic methylated sterols contribute at least in part to the deleterious effects of azole treatment on the integrity of the fungal vacuole.

\section{Vacuolar Trafficking as a Determinant of the Antifungal Efficacy of Ergosterol Biosynthesis Inhibitors}

Another major finding of this work is the impact that vacuolar dysfunction can have on the antifungal efficacy of the azoles. Normal vacuolar function depends on several trafficking pathways (201), each contributing to different extents to processes such as hyphal growth, stress resistance and virulence $(50,51,182)$. Due to the critical role of the vacuole in survival and pathogenesis of $C$. albicans and the extensive clinical use of azoles, it was of significance to evaluate how vacuolar dysfunction affects azole susceptibility in the context of vacuolar membrane trafficking. In this regard, we found that deficiency in autophagy or ALP trafficking did not cause significant changes in azole susceptibility. However, lack of the Rab GTPase Vps21p, which controls membrane fusion events at the pre-vacuolar compartment, enhanced C. albicans survival after azole exposure. Interestingly, Vps $21 p$ seems to not only impact fungal susceptibility to azoles antifungals but also other ergosterol biosynthesis inhibitors like terbinafine and amorolfine, which inhibit alternate steps along the ergosterol biosynthetic pathway. Interestingly, the effect of Vps21p on azole tolerance seems to be particular to the trafficking step that it mediates, since the azole tolerance phenotype was also observed in a pep $12 \Delta / \Delta$ mutant. Moreover, we also found that membrane fusion events at the vacuolar membrane mediated by another Rab GTPase, Ypt72p, can affect C. albicans susceptibility to azole antifungals, but to a lesser degree than Vps21p. This further highlights the importance of vacuolar function and dysfunction, and specifically vacuolar trafficking pathways, on the antifungal activity of the azoles and other ergosterol biosynthesis inhibitors. 
The mechanism by which the C. albicans vps $21 \Delta / \Delta$ mutant was tolerant to azole treatment, but more hypersensitive to other ergosterol biosynthesis inhibitors is not clear. However, by targeting alternate steps along the ergosterol biosynthetic pathway, treatment with each class of inhibitor creates a particular sterol profile that depends on the enzyme(s) being inhibited. Since intracellular sterol trafficking depends on both vesicle and non-vesicle mediated transport (247), disruption of a particular trafficking step could affect the distribution of the sterols accumulated upon drug treatment, thus enhancing or avoiding the effects that the buildup of a particularly toxic sterol has on the membranes where they are delivered. For the vps $21 \Delta / \Delta$ mutant, disruption of trafficking at the PVC could prevent the toxic sterols accumulated upon Erg11p inhibition from reaching the PVC itself or another target membrane for which the PVC is an intermediate step. However, this effect seems to be deleterious when Erg1p or Erg2p and Erg24p are targeted and presumably different sterols profiles are produced.

The tolerance phenotype of the $v p s 21 \Delta / \Delta$ mutant resembles the trailing growth phenomenon that is observed during standard azole susceptibility testing in $\sim 18 \%$ of $C$. albicans clinical isolates (145). "Trailing" isolates appear to be susceptible to azole treatment at 24 hours but can appear as azole resistant strains at the 48 hour time-point (144). The vps $21 \Delta / \Delta$ mutant not only "mimicked" the 24 vs 48 hours pattern of growth but also displayed some other features that "trailing" clinical isolates present such as enhanced growth despite ergosterol depletion and elimination of the trailing phenotype in acidic medium or upon incubation at $25^{\circ} \mathrm{C}$ or $42^{\circ} \mathrm{C}(124,143,219,221)$. Moreover, the $v p s 21 \Delta / \Delta$ mutant was susceptible to azole treatment in vivo, a classic feature of "trailing" isolates. These observations open the question of whether clinical isolates that display trailing growth have altered or abnormal endosomal trafficking. This question can be addressed by performing phenotypic analysis on trailing isolates in order to identify phenotypes similar to that of the $v p s 21 \Delta / \Delta$ mutant in response to stressors such as hypersensitivity to SDS and Congo red, delayed endocytosis $(50,51)$ and differential responses to ergosterol biosynthesis inhibitors. Nevertheless, the mechanisms underlying trailing growth in clinical isolates are far from being completely understood.

\section{Connecting the Dots: Vacuolar Trafficking, $\mathrm{Ca}^{2+}$ Homeostasis and Azole Tolerance}

The relationship between $\mathrm{Ca}^{2+}$ and membrane trafficking has been previously recognized, exemplified by the role for $\mathrm{Ca}^{2+}$ in the early stages of vesicular trafficking (248) as well as in the context of endosomal membrane trafficking and yeast homotypic vacuole fusion $(249,250)$. Two important findings of this work are that disruption of endosomal trafficking mediated by Vps $21 \mathrm{p}$ creates a $\mathrm{Ca}^{2+}$ homeostasis defect characterized by elevated levels of intracellular $\mathrm{Ca}^{2+}$, and that the tolerance of the vps $21 \Delta / \Delta$ mutant is dependent upon $\mathrm{Ca}^{2+}$ and $\mathrm{Ca}^{2+}$-dependent signaling via the serine/threonine protein phosphatase calcineurin. $\mathrm{Ca}^{2+}$ dependent signaling has been widely linked to azole tolerance in fungi (147). In addition, the vacuole is the major storage site of intracellular calcium in fungi and several proteins that regulate the $\mathrm{Ca}^{2+}$ fluxes required for calcineurin signaling are located on the vacuolar membrane (161). These include the $\mathrm{Ca}^{2+}$ pump Pmclp, the $\mathrm{Ca}^{2+} / \mathrm{H}^{+}$exchanger Vcxlp and the $\mathrm{Ca}^{2+}$ channel 
Yvc1p (161). Disruption of endosomal trafficking could potentially cause abnormal delivery of these proteins to the vacuolar membrane, thus altering $\mathrm{Ca}^{2+}$ homeostasis and contributing to azoles tolerance. However, we have determined that deletion of either $V C X 1$ or $P M C l$ (see Appendix $\mathbf{C}$ for a list of strains) does not affect C. albicans azole susceptibility or induce trailing growth under standard testing conditions (Figure 6-1A and B). This suggests that if defects in trafficking lead to a lack of Pmc1p or Vcx1p in the vacuolar membrane, they may not be sufficient to alter azole susceptibility.

Another possibility is that the mechanisms that regulate $\mathrm{Ca}^{2+}$ pumps and channels on the plasma membrane are affected by the trafficking defects of the vps $21 \Delta / \Delta$ mutant and, this in turn, leads to the $\mathrm{Ca}^{2+}$ homeostasis defects observed. The $S$. cerevisiae secretory pathway, responsible for the transport of a large number of plasma membrane and extracellular proteins, require the $\mathrm{Ca}^{2+}$ pump Pmrlp (251). Pmrlp was shown to be regulated by ESCRT components involved in membrane sorting at the PVC, including Vps28p. Mutants lacking Pmrlp or components of vesicle-mediated trafficking (161) exhibit higher rates of $\mathrm{Ca}^{2+}$ uptake due to constitutive activation of a high-affinity $\mathrm{Ca}^{2+}$ influx system (HACS) (161) formed by Cch1p and Mid1p (252), the yeast homologous of the mammalian L-type $\mathrm{Ca}^{2+}$ channel. Cch1p/Mid1p appear to ensure the uptake of $\mathrm{Ca}^{2+}$ needed by Pmrlp to sustain the secretory pathway (252). Thus, alterations in PVC membrane trafficking could potentially alter $\mathrm{Ca}^{2+}$ homeostasis via several mechanisms. Although we did not establish the mechanism responsible for the increased levels of cytoplasmic $\mathrm{Ca}^{2+}$ in the mutant, we found that the adjunctive treatment with $\mathrm{Ca}^{2+}$ channel blocker nisoldipine rendered the $v p s 21 \Delta / \Delta$ mutant sensitive to fluconazole. These results highlight the need of the mutant for an active $\mathrm{Ca}^{2+}$ channel in order tolerate azole treatment, presumably Cch $1 \mathrm{p} / \mathrm{Mid} 1 \mathrm{p}$.

We also found that calcineurin dependent gene expression in the $v p s 21 \Delta / \Delta$ mutant appears to be enhanced upon azole exposure, as indicated by the reporter assay using GFP $\gamma$ under the promoter of the calcineurin responsive gene RTA2. As expected, inhibition of calcineurin signaling with CsA or FK506 rendered the vps21 $\Delta / \Delta$ mutant susceptible to fluconazole. The role of calcineurin signaling in the mutant's azole tolerance could be explained in the models outlined in Figure 6-2 and Figure 6-3. In a "wild-type" strain (Figure 6-2), azole treatment (step 1) causes a release of $\mathrm{Ca}^{2+}$ from intracellular stores and influx from the medium (step 2). This in turn leads to activation of calcineurin signaling (step 3), followed by dephosphorylation of a calcineurin-dependent transcription factor such as Crzlp (step 4) and the expression of genes involved in stress response (step 5) and azole tolerance (step 6).

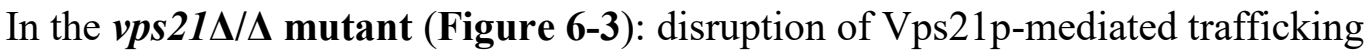
(step 1) creates a calcium homeostasis defect (i.e., elevated basal levels of cytoplasmic $\mathrm{Ca}^{2+}$ ) that may rely on a plasma membrane channel (perhaps Cch1p/Mid1p, step 2). This may affect the basal level of calcineurin activity and/or affect the magnitude or duration of calcineurin signaling (step 3) and downstream events (step 4). This could lead to enhanced expression of genes involved in stress response (step 5), which promote the mutant's survival (step 6) once it is exposed to azole antifungals (step 7). 
A.

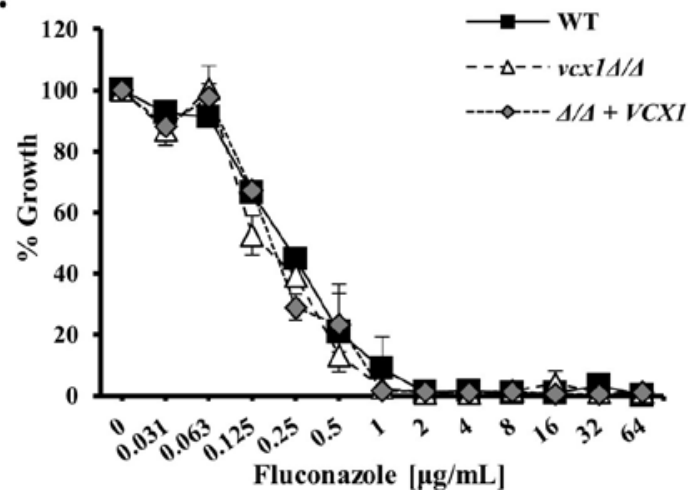

B.

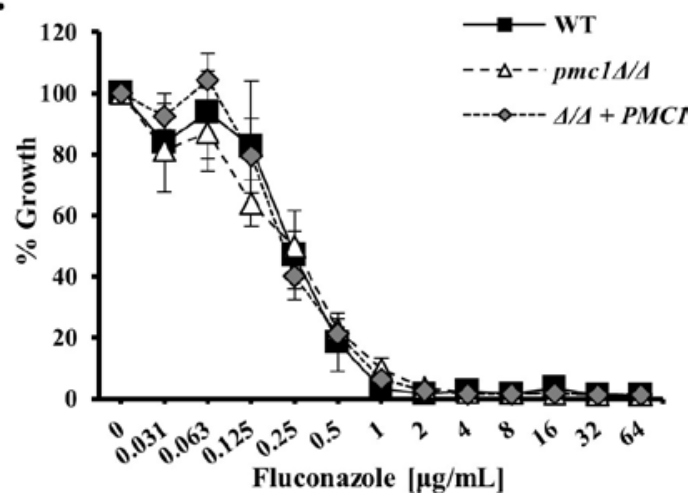

Figure 6-1. The vacuolar calcium pump Pmc1p and the $\mathrm{Ca}^{2+} / \mathrm{H}^{+}$exchanger Vex1p do not impact $C$. albicans susceptibility to fluconazole.

The susceptibility to fluconazole of the $C$. albicans $v c x l \Delta / \Delta$ (A) and $p m c l \Delta / \Delta$ mutants (B) were compared to WT (YJB6284), and 'reconstituted' control strain using the standard CLSI broth microdilution protocol. Growth is expressed as a percentage of the growth in the minus drug (DMSO) control wells. The means \pm standard deviation of two biological replicates is shown for each panel. 


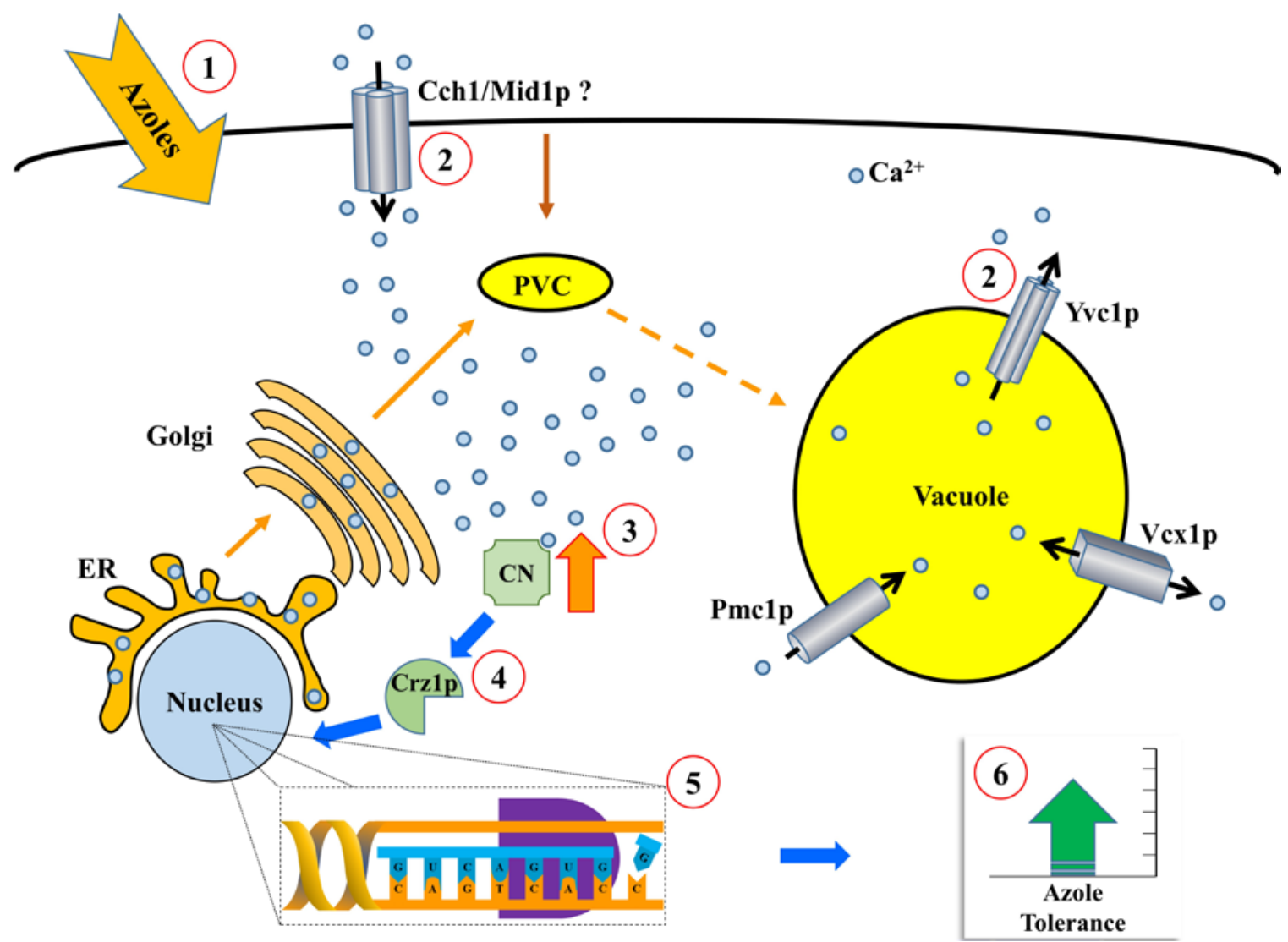

Figure 6-2. Accepted model of $\mathrm{Ca}^{2+}$ dependent response to azole antifungals in $C$. albicans.

Azole exposure (1) induces rapid fluxes of $\mathrm{Ca}^{2+}$ from the extracellular space and its release from intracellular stores into the cytoplasma (2). The elevated $\mathrm{Ca}^{2+}$ levels allow activation of the calcineurin signaling pathway ( 3 and 4 ) and the expression of number of genes (5) involved in stress response (5) and survival to azoles (6). 


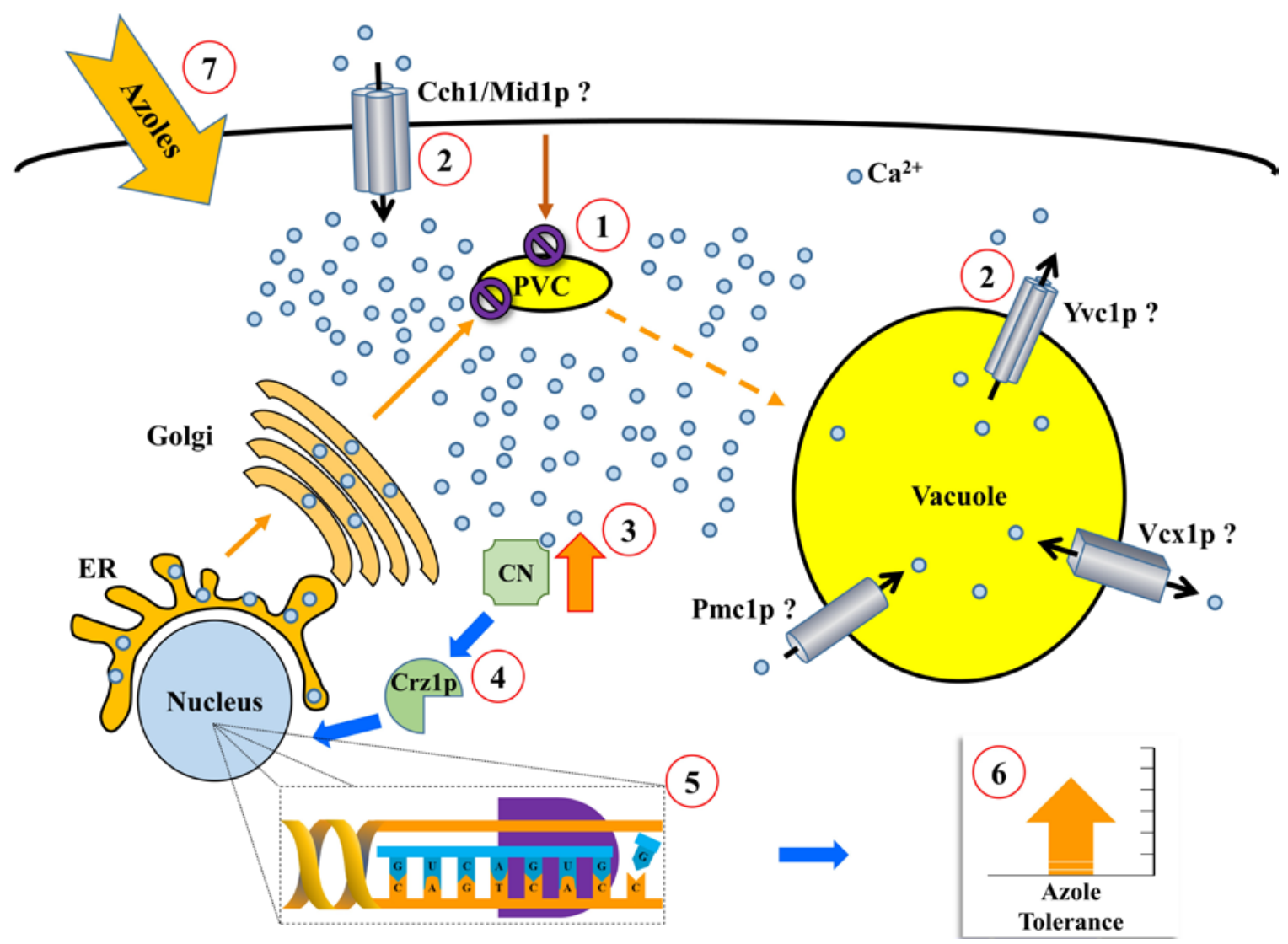

Figure 6-3. Proposed model for the $\mathrm{Ca}^{2+}$ dependent azole tolerance in the $C$. albicans vps $21 \Delta / \Delta$ mutant.

Disruption of membrane trafficking mediated by the Rab GTPase Vps21p (1) causes a $\mathrm{Ca}^{2+}$ homeostasis defect (2) represented by elevated levels of intracellular $\mathrm{Ca}^{2+}$ that may alter the intensity of calcineurin activity (3). Calcineurin-dependent transcription factors (4) then drive the enhanced expression of genes involved in stress responses (5) that support the survival of the vps21 $1 / \Delta$ mutant upon azole exposure (6 and 7). 
Although loss of Vps28p, an ESCRT component involved in MVB biogenesis at the PVC, affects $\mathrm{Ca}^{2+}$ homeostasis leading to activation of calcineurin signaling in $S$. cerevisiae, our results indicate that $\mathrm{Vps} 28 \mathrm{p}$ does not impact azole tolerance in $C$. albicans. Furthermore, it was previously reported that a $C$. albicans mutant defective in retrograde trafficking from the PVC back to the Golgi is highly sensitive to the azoles despite having upregulated calcineurin signaling (222). Taken together, these findings indicate that azole tolerance in membrane trafficking mutants does not only depend on activation of calcineurin signaling, but it is also determined by the trafficking steps blocked. In support of this notion deletion of APS3 (see Appendix $\mathbf{C}$ for a list of strains), which controls trafficking through the ALP pathway, abolished the $C$. albicans vps $21 \Delta / \Delta$ mutant's ability to withstand azole exposure (Figure 6-4) despite having no effect on the susceptibility of the "wild-type" (Figure 3-2B). Thus, the vps $21 \Delta / \Delta$ mutant's azole tolerance is not only dependent on $\mathrm{Ca}^{2+}$ and calcineurin signaling, but also relies on membrane trafficking, specifically from the Golgi to the vacuole. This further highlights the significance of membrane trafficking in the antifungal activity of azole antifungals in C. albicans.

\section{Concluding Remarks}

The work described herein revealed that a $C$. albicans vps $21 \Delta / \Delta$ mutant displays enhanced growth in response to azole treatment, resembling an exaggerated form of the trailing growth phenomenon. However, it is unclear how, or if the azole tolerant phenotype of the vps $21 \Delta / \Delta$ mutant relates to that of trailing clinical isolates, or if these clinical isolates have abnormal endosomal trafficking. This could be investigated by testing trailing clinical isolates for phenotypes indicative of endosomal trafficking defects. This would include sensitivity to SDS, Congo red, allylamines and morpholines, and delayed endocytosis $(50,51,186)$.

While deletion of VPS21 alone was not sufficient to confer a survival advantage upon $C$. albicans following azole treatment in the mouse model of vaginal candidiasis, several important questions remain. First, the azole susceptibility of the vps $21 \Delta / \Delta$ mutant could be tested in a mouse model disseminated infection. This may be important because several $C$. albicans mutants that are avirulent in the disseminated model have no apparent defects in pathogenicity in the vaginal model of infection. For example, we have recently reported that $C$. albicans lacking ERG2 or ERG24, which encode enzymes involved in ergosterol biosynthesis, are pathogenic in a mouse model of vaginal infection but not in the mouse model of disseminated candidiasis (172). Second, as the fungistatic azoles depend upon the host's immune defenses to clear fungal infections, it will be important to determine if the $C$. albicans vps $21 \Delta / \Delta$ mutant have a survival advantage over the wildtype following azole treatment in immunocompromised hosts. This can be tested by conducting similar experiments to those described herein but using immunosuppressed mice. Third, it is crucial to determine if cells that persist following azole exposure, such as the $v p s 21 \Delta / \Delta$ mutant and trailing isolates, are more predisposed to develop azole resistance overtime. This can be tested by exposing both wild-type and vps $21 \Delta / \Delta$ mutant or trailing isolates to increasing concentrations of azole antifungals and comparing the 
frequency of appearance of azole resistance in each passage. Finally, it was also revealed that the C. albicans vps $21 \Delta / \Delta$ mutant has elevated basal levels of $\mathrm{Ca}^{2+}$ and enhanced calcineurin-dependent transcriptional response following azole exposure. It will also be important to determine if differences in basal levels of intracellular free $\mathrm{Ca}^{2+}$ and azoleinduced $\mathrm{Ca}^{2+}$-dependent signaling contribute to variations in azole susceptibility in clinical isolates. This can be tested by using several of the methodologies described in this work. 


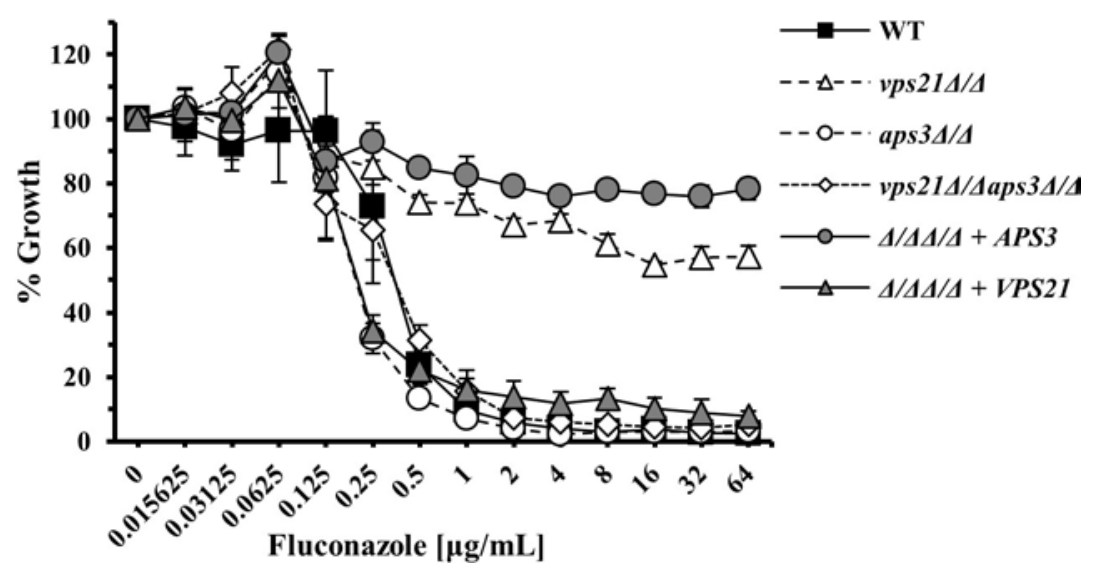

Figure 6-4. Disruption of the ALP trafficking pathway in the $C$. albicans vps21 $\Delta / \Delta$ mutant eliminates azole tolerance.

The susceptibility to fluconazole of the vps21 $1 / \Delta$ aps $3 \Delta / \Delta$ double mutant was compared to that of the vps $21 \Delta / \Delta$ mutant, aps $3 \Delta / \Delta$, YJB6284 (WT), and 'reconstituted' control strains using the standard CLSI broth microdilution protocol. Growth was measured as OD600nm and expressed as a percentage of the DMSO control wells. The mean \pm standard deviation of two biological replicates is shown. 


\section{LIST OF REFERENCES}

1. Hawksworth DL. 2001. The magnitude of fungal diversity: the 1.5 million species estimate revisited. Mycol Res 105:1422-1432.

2. Garcia-Solache MA, Casadevall A. 2010. Global Warming Will Bring New Fungal Diseases for Mammals. mBio 1.

3. Taylor LH, Latham SM, Woolhouse ME. 2001. Risk factors for human disease emergence. Philos Trans R Soc Lond B Biol Sci 356:983-989.

4. Pfaller MA, Diekema DJ. 2007. Epidemiology of invasive candidiasis: a persistent public health problem. Clin Microbiol Rev 20:133-163.

5. Vandeputte P, Ferrari S, Coste AT. 2012. Antifungal resistance and new strategies to control fungal infections. Int J Microbiol 2012:713687.

6. Brown GD, Denning DW, Gow NA, Levitz SM, Netea MG, White TC. 2012. Hidden killers: human fungal infections. Sci Transl Med 4:165rv113.

7. Casadevall A. 2012. Fungi and the rise of mammals. PLoS Pathog 8:e1002808.

8. Romani L. 2011. Immunity to fungal infections. Nat Rev Immunol 11:275-288.

9. Ho KM, Cheng TS. 2010. Common Superficial Fungal Infections - a Short Review. Hong Kong Med D 15:23-27.

10. Elewski BE. 1998. Onychomycosis: Pathogenesis, Diagnosis, and Management. Clin Microbiol Rev 11:415-429.

11. Thomas J, Jacobson GA, Narkowicz CK, Peterson GM, Burnet H, Sharpe C. 2010. Toenail onychomycosis: an important global disease burden. J Clin Pharm Ther 35:497-519.

12. Pang KR, Wu JJ, Huang DB, K. Tyring S. 2004. Subcutaneous fungal infections. Dermatol Ther 17:523-531.

13. Menzin J, Meyers JL, Friedman M, Perfect JR, Langston AA, Danna RP, Papadopoulos G. 2009. Mortality, length of hospitalization, and costs associated with invasive fungal infections in high-risk patients. Am J Health Syst Pharm 66:1711-1717.

14. Barber GR, Brown AE, Kiehn TE, Edwards FF, Armstrong D. 1993. Catheter-related Malassezia furfur fungemia in immunocompromised patients. Am J Med 95:365-370.

15. Kabangila R, Semvua K, Rambau P, Jackson K, Mshana SE, Jaka H, Peck RN. 2011. Pulmonary histoplasmosis presenting as chronic productive cough, fever, and massive unilateral consolidation in a 15-year-old immune-competent boy: a case report. J Med Case Rep 5:374.

16. Petrikkos G, Skiada A, Lortholary O, Roilides E, Walsh TJ, Kontoyiannis DP. 2012. Epidemiology and clinical manifestations of mucormycosis. Clin Infect Dis 54 Suppl 1:S23-34.

17. Kontoyiannis DP, Marr KA, Park BJ, Alexander BD, Anaissie EJ, Walsh TJ, Ito J, Andes DR, Baddley JW, Brown JM, Brumble LM, Freifeld AG, Hadley S, Herwaldt LA, Kauffman CA, Knapp K, Lyon GM, Morrison VA, Papanicolaou G, Patterson TF, Perl TM, Schuster MG, Walker R, Wannemuehler KA, Wingard JR, Chiller TM, Pappas PG. 2010. Prospective surveillance for invasive fungal infections in hematopoietic stem cell transplant 
recipients, 2001-2006: overview of the Transplant-Associated Infection Surveillance Network (TRANSNET) Database. Clin Infect Dis 50:1091-1100.

18. Kullberg BJ, Arendrup MC. 2015. Invasive Candidiasis. N Engl J Med 373:1445-1456.

19. Enoch DA, Ludlam HA, Brown NM. 2006. Invasive fungal infections: a review of epidemiology and management options. J Med Microbiol 55:809-818.

20. Barnes PD, Marr KA. 2006. Aspergillosis: spectrum of disease, diagnosis, and treatment. Infect Dis Clin North Am 20:545-561, vi.

21. Denning DW, O'Driscoll BR, Hogaboam CM, Bowyer P, Niven RM. 2006. The link between fungi and severe asthma: a summary of the evidence. Eur Respir J 27:615-626.

22. Menzies D, Holmes L, McCumesky G, Prys-Picard C, Niven R. 2011. Aspergillus sensitization is associated with airflow limitation and bronchiectasis in severe asthma. Allergy 66:679-685.

23. Denning DW, Pleuvry A, Cole DC. 2013. Global burden of allergic bronchopulmonary aspergillosis with asthma and its complication chronic pulmonary aspergillosis in adults. Med Mycol 51:361-370.

24. Botts MR, Hull CM. 2010. Dueling in the lung: how Cryptococcus spores race the host for survival. Curr Opin Microbiol 13:437-442.

25. Lin X. 2009. Cryptococcus neoformans: morphogenesis, infection, and evolution. Infect Genet Evol 9:401-416.

26. Park BJ, Wannemuehler KA, Marston BJ, Govender N, Pappas PG, Chiller TM. 2009. Estimation of the current global burden of cryptococcal meningitis among persons living with HIV/AIDS. AIDS 23:525-530.

27. Teles F, Seixas J. 2015. The future of novel diagnostics in medical mycology. J Med Microbiol 64:315-322.

28. Chandrasekar P. 2010. Diagnostic challenges and recent advances in the early management of invasive fungal infections. Eur J Haematol 84:281-290.

29. Arvanitis M, Anagnostou T, Fuchs BB, Caliendo AM, Mylonakis E. 2014. Molecular and nonmolecular diagnostic methods for invasive fungal infections. Clin Microbiol Rev 27:490-526.

30. Lu Y, Su C, Liu H. 2014. Candida albicans hyphal initiation and elongation. Trends Microbiol 22:707-714.

31. Berman J. 2006. Morphogenesis and cell cycle progression in Candida albicans. Curr Opin Microbiol 9:595-601.

32. Calderone RA, Fonzi WA. 2001. Virulence factors of Candida albicans. Trends Microbiol 9:327-335.

33. Hurley R, De Louvois J. 1979. Candida vaginitis. Postgrad Med J 55:645-647.

34. Sobel JD. 2007. Vulvovaginal candidosis. Lancet 369:1961-1971.

35. Pappas PG, Rex JH, Sobel JD, Filler SG, Dismukes WE, Walsh TJ, Edwards JE. 2004. Guidelines for Treatment of Candidiasis. Clin Infect Dis 38:161-189.

36. Sobel JD, Faro S, Force RW, Foxman B, Ledger WJ, Nyirjesy PR, Reed BD, Summers PR. 1998. Vulvovaginal candidiasis: Epidemiologic, diagnostic, and therapeutic considerations. Am J Obstet Gynecol 178:203-211.

37. Samaranayake LP, Keung Leung W, Jin L. 2009. Oral mucosal fungal infections. Periodontol 2000 49:39-59. 
38. Grohskopf LA, Andriole VT. 1996. Systemic Candida infections. Yale J Biol Med 69:505-515.

39. Morgan J. 2005. Global trends in candidemia: Review of reports from 19952005. Curr Infect Dis Rep 7:429-439.

40. Wisplinghoff H, Bischoff T, Tallent SM, Seifert H, Wenzel RP, Edmond MB. 2004. Nosocomial bloodstream infections in US hospitals: analysis of 24,179 cases from a prospective nationwide surveillance study. Clin Infect Dis 39:309317.

41. Miller LG, Hajjeh RA, Edwards JE. 2001. Estimating the Cost of Nosocomial Candidemia in the United States. Clin Infect Dis 32:1110.

42. Mayer FL, Wilson D, Hube B. 2013. Candida albicans pathogenicity mechanisms. Virulence 4:119-128.

43. Romani L, Bistoni F, Puccetti P. 2003. Adaptation of Candida albicans to the host environment: the role of morphogenesis in virulence and survival in mammalian hosts. Curr Opin Microbiol 6:338-343.

44. Sudbery P, Gow N, Berman J. 2004. The distinct morphogenic states of Candida albicans. Trends Microbiol 12:317-324.

45. Sudbery PE. 2011. Growth of Candida albicans hyphae. Nat Rev Microbiol 9:737-748.

46. Saville SP, Lazzell AL, Monteagudo C, Lopez-Ribot JL. 2003. Engineered Control of Cell Morphology In Vivo Reveals Distinct Roles for Yeast and Filamentous Forms of Candida albicans during Infection. Eukaryot Cell 2:10531060.

47. Berman J, Sudbery PE. 2002. Candida albicans: a molecular revolution built on lessons from budding yeast. Nat Rev Genet 3:918-930.

48. Lu Y, Su C, Wang A, Liu H. 2011. Hyphal development in Candida albicans requires two temporally linked changes in promoter chromatin for initiation and maintenance. PLoS Biol 9:e1001105.

49. Palmer GE, Cashmore A, Sturtevant J. 2003. Candida albicans VPS11 Is Required for Vacuole Biogenesis and Germ Tube Formation. Eukaryot Cell 2:411-421.

50. Palmer GE. 2010. Endosomal and AP-3-dependent vacuolar trafficking routes make additive contributions to Candida albicans hyphal growth and pathogenesis. Eukaryot Cell 9:1755-1765.

51. Johnston DA, Eberle KE, Sturtevant JE, Palmer GE. 2009. Role for endosomal and vacuolar GTPases in Candida albicans pathogenesis. Infect Immun 77:2343-2355.

52. Lo H-J, Köhler JR, DiDomenico B, Loebenberg D, Cacciapuoti A, Fink GR. 1997. Nonfilamentous C. albicans Mutants Are Avirulent. Cell 90:939-949.

53. Murad AMA, Leng P, Straffon M, Wishart J, Macaskill S, MacCallum D, Schnell N, Talibi D, Marechal D, Tekaia F, d'Enfert C, Gaillardin C, Odds FC, Brown AJP. 2001. NRG1 represses yeast-hypha morphogenesis and hyphaspecific gene expression in Candida albicans. EMBO J 20:4742-4752.

54. Cleary IA, Reinhard SM, Lazzell AL, Monteagudo C, Thomas DP, LopezRibot JL, Saville SP. 2016. Examination of the pathogenic potential of Candida 
albicans filamentous cells in an animal model of haematogenously disseminated candidiasis. FEMS Yeast Res 16.

55. Lionakis MS, Lim JK, Lee CC, Murphy PM. 2011. Organ-specific innate immune responses in a mouse model of invasive candidiasis. J Innate Immun 3:180-199.

56. Naglik JR, Challacombe SJ, Hube B. 2003. Candida albicans Secreted Aspartyl Proteinases in Virulence and Pathogenesis. Microbiol Mol Biol Rev 67:400-428.

57. Monod M, Togni G, Hube B, Sanglard D. 1994. Multiplicity of genes encoding secreted aspartic proteinases in Candida species. Mol Microbiol 13:357-368.

58. Monod M, Hube B, Hess D, Sanglard D. 1998. Differential regulation of SAP8 and $S A P 9$, which encode two new members of the secreted aspartic proteinase family in Candida albicans. Microbiology 144:2731-2737.

59. de Repentigny L, Aumont F, Bernard K, Belhumeur P. 2000. Characterization of Binding of Candida albicans to Small Intestinal Mucin and Its Role in Adherence to Mucosal Epithelial Cells. Infect Immun 68:3172-3179.

60. Kaminishi H, Miyaguchi H, Tamaki T, Suenaga N, Hisamatsu M, Mihashi I, Matsumoto H, Maeda H, Hagihara Y. 1995. Degradation of humoral host defense by Candida albicans proteinase. Infect Immun 63:984-988.

61. Rüchel R. 1986. Cleavage of immunoglobulins by pathogenic yeasts of the genus Candida. Microbiol Sci 3:316-319.

62. Pericolini E, Gabrielli E, Amacker M, Kasper L, Roselletti E, Luciano E, Sabbatini S, Kaeser M, Moser C, Hube B, Vecchiarelli A, Cassone A. 2015. Secretory Aspartyl Proteinases Cause Vaginitis and Can Mediate Vaginitis Caused by Candida albicans in Mice. mBio 6:e00724.

63. Beauséjour A, Grenier D, Goulet J-P, Deslauriers N. 1998. Proteolytic Activation of the Interleukin-1 $\beta$ Precursor by Candida albicans. Infect Immun 66:676-681.

64. Hube B, Sanglard D, Odds FC, Hess D, Monod M, Schäfer W, Brown AJ, Gow NA. 1997. Disruption of each of the secreted aspartyl proteinase genes $S A P 1, S A P 2$, and $S A P 3$ of Candida albicans attenuates virulence. Infect Immun 65:3529-3538.

65. Sanglard D, Hube B, Monod M, Odds FC, Gow NA. 1997. A triple deletion of the secreted aspartyl proteinase genes SAP4, SAP5, and SAP6 of Candida albicans causes attenuated virulence. Infect Immun 65:3539-3546.

66. Schaller M, Schäfer W, Korting HC, Hube B. 1998. Differential expression of secreted aspartyl proteinases in a model of human oral candidosis and in patient samples from the oral cavity. Mol Microbiol 29:605-615.

67. Schaller M, Korting HC, Schäfer W, Bastert J, Chen W, Hube B. 1999. Secreted aspartic proteinase (Sap) activity contributes to tissue damage in a model of human oral candidosis. Mol Microbiol 34:169-180.

68. De Bernardis F, Arancia S, Morelli L, Hube B, Sanglard D, Sch, xe, fer W, Cassone A. 1999. Evidence That Members of the Secretory Aspartyl Proteinase Gene Family, in Particular SAP2, Are Virulence Factors for Candida Vaginitis. J Infect Dis 179:201-208. 
69. Cassone A, Bernardis FD, Mondello F, Ceddia T, Agatensi L. 1987. Evidence for a Correlation Between Proteinase Secretion and Vulvovaginal Candidosis. J Infect Dis 156:777-783.

70. Agatensi L, Franchi F, Mondello F, Bevilacqua RL, Ceddia T, De Bernardis F, Cassone A. 1991. Vaginopathic and proteolytic Candida species in outpatients attending a gynaecology clinic. J Clin Pathol 44:826-830.

71. de Bernardis F, Boccanera M, Rainaldi L, Guerra CE, Quinti I, Cassone A. 1992. The secretion of aspartyl proteinase, a virulence enzyme, by isolates of Candida albicans from the oral cavity of HIV-infected subjects. Eur J Epidemiol 8:362-367.

72. Bernardis FD, Agatensi L, Ross IK, Emerson GW, Lorenzini R, Sullivan PA, Cassone A. 1990. Evidence for a Role for Secreted Aspartate Proteinase of Candida albicans in Vulvovaginal Candidiasis. J Infect Dis 161:1276-1283.

73. Leidich SD, Ibrahim AS, Fu Y, Koul A, Jessup C, Vitullo J, Fonzi W, Mirbod F, Nakashima S, Nozawa Y, Ghannoum MA. 1998. Cloning and Disruption of caPLB1, a Phospholipase B Gene Involved in the Pathogenicity of Candida albicans. J Biol Chem 273:26078-26086.

74. Niewerth M, Korting HC. 2001. Phospholipases of Candida albicans. Mycoses 44:361-367.

75. Schaller M, Borelli C, Korting HC, Hube B. 2005. Hydrolytic enzymes as virulence factors of Candida albicans. Mycoses 48:365-377.

76. Mukherjee PK, Seshan KR, Leidich SD, Chandra J, Cole GT, Ghannoum MA. 2001. Reintroduction of the PLB1 gene into Candida albicans restores virulence in vivo. Microbiology 147:2585-2597.

77. Ghannoum MA. 2000. Potential Role of Phospholipases in Virulence and Fungal Pathogenesis. Clin Microbiol Rev 13:122-143.

78. Georgopapadakou NH, Walsh TJ. 1996. Antifungal agents chemotherapeutic targets and immunologic strategies. Antimicrob Agents Chemother 40:279-291.

79. Zhang YQ, Gamarra S, Garcia-Effron G, Park S, Perlin DS, Rao R. 2010. Requirement for ergosterol in V-ATPase function underlies antifungal activity of azole drugs. PLoS Pathog 6:e1000939.

80. Abe F, Usui K, Hiraki T. 2009. Fluconazole modulates membrane rigidity, heterogeneity, and water penetration into the plasma membrane in Saccharomyces cerevisiae. Biochemistry 48:8494-8504.

81. Lupetti A, Danesi R, Campa MDT, Mario, Kelly S. 2002. Molecular basis of resistance to azole antifungals. Trends Mol Med 8:76-81.

82. Umebayashi K, Nakano A. 2003. Ergosterol is required for targeting of tryptophan permease to the yeast plasma membrane. J Cell Biol 161:1117-1131.

83. Sanglard D, White TC. 2006. Molecular Principles of Antifungal Drug Resistance, p 197-212. In Heitman J, Filler SG, Edwards JE, Mitchell AP (ed), Molecular Principles of Fungal Pathogenesis. ASM Press.

84. Altmann K, Westermann B. 2005. Role of essential genes in mitochondrial morphogenesis in Saccharomyces cerevisiae. Mol Biol Cell 16:5410-5417.

85. Kobayashi D, Kondo K, Uehara N, Otokozawa S, Tsuji N, Yagihashi A, Watanabe N. 2002. Endogenous Reactive Oxygen Species Is an Important 
Mediator of Miconazole Antifungal Effect. Antimicrob Agents Chemother 46:3113-3117.

86. Portillo F, Gancedo C. 1984. Mode of action of miconazole on yeasts: inhibition of the mitochondrial ATPase. Eur J Biochem 143:273-276.

87. Zhang Y-Q, Rao R. 2010. Beyond ergosterol: Linking $\mathrm{pH}$ to antifungal mechanisms. Virulence 1:551-554.

88. Mercer EI. 1991. Morpholine antifungals and their mode of action. Biochem Soc Trans 19:788-793.

89. Polak AM. 1992. Preclinical data and mode of action of amorolfine. Clin Exp Dermatol 17:8-12.

90. Ghannoum MA, Rice LB. 1999. Antifungal Agents: Mode of Action, Mechanisms of Resistance, and Correlation of These Mechanisms with Bacterial Resistance. Clin Microbiol Rev 12:501-517.

91. Odds FC, Brown AJP, Gow NAR. 2003. Antifungal agents: mechanisms of action. Trends Microbiol 11:272-279.

92. Sheehan DJ, Hitchcock CA, Sibley CM. 1999. Current and Emerging Azole Antifungal Agents. Clin Microbiol Rev 12:40-79.

93. Maertens JA. 2004. History of the development of azole derivatives. Clin Microbiol Infect 10:1-10.

94. Rajendran R, Mowat E, McCulloch E, Lappin DF, Jones B, Lang S, Majithiya JB, Warn P, Williams C, Ramage G. 2011. Azole resistance of Aspergillus fumigatus biofilms is partly associated with efflux pump activity. Antimicrob Agents Chemother 55:2092-2097.

95. Zonios DI, Bennett JE. 2008. Update on Azole Antifungals. Semin Respir Crit Care Med 29:198-210.

96. Gray KC, Palacios DS, Dailey I, Endo MM, Uno BE, Wilcock BC, Martin BD. 2012. Amphotericin primarily kills yeast by simply binding ergosterol. Proc Natl Acad Sci U S A 109:2234-2239.

97. te Welscher YM, Jones L, van Leeuwen MR, Dijksterhuis J, de Kruijff B, Eitzen G, Breukink E. 2010. Natamycin inhibits vacuole fusion at the priming phase via a specific interaction with ergosterol. Antimicrob Agents Chemother 54:2618-2625.

98. te Welscher YM, ten Napel HH, Balague MM, Souza CM, Riezman H, de Kruijff B, Breukink E. 2008. Natamycin blocks fungal growth by binding specifically to ergosterol without permeabilizing the membrane. J Biol Chem 283:6393-6401.

99. Chen WC, Chou D-L, Feingold DS. 1978. Dissociation Between Ion Permeability and the Lethal Action of Polyene Antibiotics on Candida albicans. Antimicrob Agents Chemother 13:914-917.

100. De Kruijff B, Gerritsen WJ, Oerlemans A, Van Dijck PWM, Demel RA, Van Deenen LLM. 1974. Polyene antibiotic-sterol interactions in membranes of Acholeplasma laidlawii cells and lecithin liposomes. II. Temperature dependence of the polyene antibiotic-sterol complex formation. Biochim Biophys Acta 339:44-56.

101. Bolard J. 1986. How do the polyene macrolide antibiotics affect the cellular membrane properties? Biochim Biophys Acta 864:257-304. 
102. Loeffler J, Stevens DA. 2003. Antifungal Drug Resistance. Clin Infect Dis 36:S31-41.

103. Laniado-Laborin R, Cabrales-Vargas MN. 2009. Amphotericin B: side effects and toxicity. Rev Iberoam Micol 26:223-227.

104. Patterson TF. 2007. The role of echinocandins, extended-spectrum azoles, and polyenes to treat opportunistic moulds and Candida. Curr Infect Dis Rep 1:5-11.

105. Nishiyama Y, Uchida K, Yamaguchi H. 2002. Morphological changes of Candida albicans induced by micafungin (FK463), a water-soluble echinocandinlike lipopeptide. J Electron Microsc 51:247-255.

106. Chandrasekar PH, Sobel JD. 2006. Micafungin: A New Echinocandin. Clin Infect Dis 42:1171-1178.

107. Kanafani ZA, Perfect JR. 2008. Resistance to Antifungal Agents: Mechanisms and Clinical Impact. Clin Infect Dis 46:120-128.

108. Denning DW. 2003. Echinocandin antifungal drugs. Lancet 362:1142-1151.

109. Tawara S, Ikeda F, Maki K, Morishita Y, Otomo K, Teratani N, Goto T, Tomishima M, Ohki H, Yamada A, Kawabata K, Takasugi H, Sakane K, Tanaka H, Matsumoto F, Kuwahara S. 2000. In Vitro Activities of a New Lipopeptide Antifungal Agent, FK463, against a Variety of Clinically Important Fungi. Antimicrob Agent Chemother 44:57-62.

110. Uchida K, Nishiyama Y, Yokota N, Yamaguchi H. 2000. In vitro antifungal activity of a novel lipopeptide antifungal agent, FK463, against various fungal pathogens. J Antibiot 53:1175-1181.

111. Nakai T, Uno J, Otomo K, Ikeda F, Tawara S, Goto T, Nishimura K, Miyaji M. 2002. In vitro Activity of FK463, a Novel Lipopeptide Antifungal Agent, against a Variety of Clinically Important Molds. Chemotherapy 48:78-81.

112. Chen SC, Slavin MA, Sorrell TC. 2011. Echinocandin Antifungal Drugs in Fungal Infections: A Comparison. Drugs 71:11-41.

113. Déry M, Hasbun R. 2011. Fluconazole-Resistant Candida: Mechanisms and Risk Factor Identification. Curr Fungal Infect Rep 5:23-28.

114. Rex JH, Rinaldi MG, Pfaller MA. 1995. Resistance of Candida Species to Fluconazole. Antimicrob Agent Chemother 39:1-8.

115. Alves SH, Lopes JO, Costa JM, Klock C. 1997. Development of Secondary Resistance to Fluconazole in Cryptococcus neoformans Isolated from a Patient with AIDS. Rev Inst Med Trop Sao Paulo 39:359-362.

116. Alanio A, Cordonnier C, Bretagne S. 2011. Azole Resistance in Aspergillus fumigatus-Current Epidemiology and Future Perspectives. Curr Fungal Infect Rep 5:168-178.

117. Papon N, Courdavault V, Clastre M, Bennett RJ. 2013. Emerging and Emerged Pathogenic Candida Species: Beyond the Candida albicans Paradigm. PLoS Pathog 9:1-4.

118. Chakrabarti A, Chatterjee SS, Rao KL, Zameer MM, Shivaprakash MR, Singhi S, Singh R, Varma SC. 2009. Recent experience with fungaemia: change in species distribution and azole resistance. Scand J Infect Dis 41:275-284.

119. Verweij PE, Mellado E, Melchers WJG. 2007. Multiple-Triazole-Resistant Aspergillosis. N Engl J Med 356:1481-1483. 
120. Verweij PE, Snelders E, Kema GHJ, Mellado E, Melchers WJG. 2009. Azole resistance in Aspergillus fumigatus: a side-effect of environmental fungicide use? Lancet Infect Dis 9:789-795.

121. Lopez-Ribot JL, McAtee RK, Lee LN, Kirkpatrick WR, White TC, Sanglard D, Patterson TF. 1998. Distinct Patterns of Gene Expression Associated with Development of Fluconazole Resistance in Serial Candida albicans Isolates from Human Immunodeficiency Virus-Infected Patients with Oropharyngeal Candidiasis. Antimicrob Agent Chemother 42:2932-2937.

122. White TC, Holleman S, Dy F, Mirels LF, Stevens DA. 2002. Resistance Mechanisms in Clinical Isolates of Candida albicans. Antimicrob Agent Chemother 46:1704-1713.

123. Lee MK, Williams LE, Warnock DW, Arthington-Skaggs BA. 2004. Drug resistance genes and trailing growth in Candida albicans isolates. J Antimicrob Chemother 53:217-224.

124. Marr KA, Rustad TR, Rex JH, White TC. 1999. The Trailing End Point Phenotype in Antifungal Suceptibility Testing is $\mathrm{pH}$ Dependent. Antimicrob Agent Chemother 43:1383-1386.

125. White TC. 1997. Increased mRNA levels of ERG16, CDR, and MDR1 correlate with increases in azole resistance in Candida albicans isolates from a patient infected with human immunodeficiency virus. Antimicrob Agent Chemother 41:1482-1487.

126. Pao SS, Paulsen IT, Saier MH. 1998. Major Facilitator Superfamily. Microbiol Mol Biol Rev 62:1-34.

127. Driessen AJM, Rosen BP, Konings WN. 2000. Diversity of transport mechanisms: common structural principles. Trends Biochem Sci 25:397-401.

128. Perea S, Lopez-Ribot JL, Kirkpatrick WR, McAtee RK, Santillan RA, Martinez M, Calabrese D, Sanglard D, Patterson TF. 2001. Prevalence of molecular mechanisms of resistance to azole antifungal agents in Candida albicans strains displaying high-level fluconazole resistance isolated from human immunodeficiency virus-infected patients. Antimicrob Agent Chemother 45:2676-2684.

129. Vanden Bossche H, Dromer F, Improvisi I, Lozano-Chiu M, Rex JH, Sanglard D. 1998. Antifungal drug resistance in pathogenic fungi. Med Mycol 36 Suppl 1:119-128.

130. Lyons CN, White TC. 2000. Transcriptional Analyses of Antifungal Drug Resistance in Candida albicans. Antimicrob Agent Chemother 44:2296-2303.

131. Coste A, Turner V, Ischer F, Morschhauser J, Forche A, Selmecki A, Berman J, Bille J, Sanglard D. 2006. A mutation in Tac1p, a transcription factor regulating $C D R 1$ and $C D R 2$, is coupled with loss of heterozygosity at chromosome 5 to mediate antifungal resistance in Candida albicans. Genetics 172:2139-2156.

132. Morschhauser J, Barker KS, Liu TT, Bla BWJ, Homayouni R, Rogers PD. 2007. The transcription factor Mrrlp controls expression of the MDRl efflux pump and mediates multidrug resistance in Candida albicans. PLoS Pathog 3:e164. 
133. Lohberger A, Coste AT, Sanglard D. 2014. Distinct Roles of Candida albicans Drug Resistance Transcription Factors TAC1, MRR1, and UPC2 in Virulence. Eukaryot Cell 13:127-142.

134. Henry KW, Nickels JT, Edlind TD. 2000. Upregulation of $E R G$ Genes in Candida Species by Azoles and Other Sterol Biosynthesis Inhibitors. Antimicrob Agent Chemother 44:2693-2700.

135. MacPherson S, Akache B, Weber S, De Deken X, Raymond M, Turcotte B. 2005. Candida albicans zinc cluster protein Upc2p confers resistance to antifungal drugs and is an activator of ergosterol biosynthetic genes. Antimicrob Agents Chemother 49:1745-1752.

136. Silver PM, Oliver BG, White TC. 2004. Role of Candida albicans transcription factor Upc2p in drug resistance and sterol metabolism. Eukaryot Cell 3:13911397.

137. Dunkel N, Liu TT, Barker KS, Homayouni R, Morschhauser J, Rogers PD. 2008. A gain-of-function mutation in the transcription factor Upc2p causes upregulation of ergosterol biosynthesis genes and increased fluconazole resistance in a clinical Candida albicans isolate. Eukaryot Cell 7:1180-1190.

138. Heilmann CJ, Schneider S, Barker KS, Rogers PD, Morschhauser J. 2010. An A643T mutation in the transcription factor Upc2p causes constitutive ERG11 upregulation and increased fluconazole resistance in Candida albicans. Antimicrob Agents Chemother 54:353-359.

139. Warrilow AG, Martel CM, Parker JE, Melo N, Lamb DC, Nes WD, Kelly DE, Kelly SL. 2010. Azole binding properties of Candida albicans sterol 14alpha demethylase (CaCYP51). Antimicrob Agents Chemother 54:4235-4245.

140. Franz R, Kelly SL, Lamb DC, Kelly DE, Ruhnke M, Morschhäuser J. 1998. Multiple Molecular Mechanisms Contribute to a Stepwise Development of Fluconazole Resistance in Clinical Candida albicans Strains. Antimicrob Agent Chemother 42:3065-3072.

141. Kelly SL, Lamb DC, Corran AJ, Baldwin BC, Kelly DE. 1995. Mode of Action and Resistance to Azole Antifungals Associated with the Formation of 14 $\alpha$-Methylergosta-8,24(28)-dien-3 $\beta, 6 \alpha$-diol. Biochem Biophys Res Commun 207:910-915.

142. Martel CM, Parker JE, Bader O, Weig M, Gross U, Warrilow AG, Rolley N, Kelly DE, Kelly SL. 2010. Identification and characterization of four azoleresistant erg3 mutants of Candida albicans. Antimicrob Agents Chemother 54:4527-4533.

143. Arthington-Skaggs BA, Warnock DW, Morrison CJ. 2000. Quantitation of Candida albicans Ergosterol Content Improves the Correlation between In Vitro Antifungal Susceptibility Test Results and In Vivo Outcome. Antimicrob Agent Chemother 44:2081-2085.

144. Rex JH, Nelson PW, Paetznick VL, Lozano-Chiu M, Espinel-Ingroff A, Anaissie EJ. 1998. Optimizing the Correlation between Results of Testing In Vitro and Therapeutic Outcome In Vivo for Fluconazole by Testing Critical Isolates in a Murine Model of Invasive Candidiasis. Antimicrob Agent Chemother 42:129-134. 
145. Arthington-Skaggs BA, Lee-Yang W, Ciblak MA, Frade JP, Brandt ME, Hajjeh RA, Harrison LH, Sofair AN, Warnock aDW. 2002. Comparison of Visual and Spectrophotometric Methods of Broth Microdilution MIC End Point Determination and Evaluation of a Sterol Quantitation Method for In Vitro Susceptibility Testing of Fluconazole and Itraconazole against Trailing and Nontrailing Candida Isolates. Antimicrob Agent Chemother 46:2477-2481.

146. Reedy JL, Filler SG, Heitman J. 2010. Elucidating the Candida albicans calcineurin signaling cascade controlling stress response and virulence. Fungal Genet Biol 47:107-116.

147. Sanglard D, Ischer F, Marchetti O, Entenza J, Bille J. 2003. Calcineurin A of Candida albicans: involvement in antifungal tolerance, cell morphogenesis and virulence. Mol Microbiol 48:959-976.

148. Sugiura R, Sio SO, Shuntoh H, Kuno T. 2002. Calcineurin phosphatase in signal transduction: lessons from fission yeast. Genes Cells 7:619-627.

149. Marchetti O, Entenza JM, Sanglard D, Bille J, Glauser MP, Moreillon P. 2000. Fluconazole plus Cyclosporine: a Fungicidal Combination Effective against Experimental Endocarditis Due to Candida albicans. Antimicrob Agent Chemother 44:2932-2938.

150. Cruz MC, Goldstein AL, Blankenship JR, Del Poeta M, Perfect JR, McCusker JH, Heitman J. 2002. Calcineurin is essential for survival during membrane stress in Candida albicans. EMBO J 21:546-559.

151. Karababa M, Valentino E, Pardini G, Coste AT, Bille J, Sanglard D. 2006. CRZ1, a target of the calcineurin pathway in Candida albicans. Mol Microbiol 59:1429-1451.

152. Edlind T, Smith L, Henry K, Katiyar S, Nickels J. 2002. Antifungal activity in Saccharomyces cerevisiae is modulated by calcium signalling. Mol Microbiol 46:257-268.

153. Cowen LE, Singh SD, Kohler JR, Collins C, Zaas AK, Schell WA, Aziz H, Mylonakis E, Perfect JR, Whitesell L, Lindquist S. 2009. Harnessing Hsp90 function as a powerful, broadly effective therapeutic strategy for fungal infectious disease. Proc Natl Acad Sci U S A 106:2818-2823.

154. Alves de Castro P, Chiaratto J, Winkelstroter LK, Pedro VL, Zambelli LN, Goldman MS, Brown NA, Goldman GH. 2014. The Involvement of the Mid1/Cch1/Yvc1 Calcium Channels in Aspergillus fumigatus Virulence. PLoS One 9.

155. Richards A, Gow NA, Veses V. 2012. Identification of vacuole defects in fungi. J Microbiol Methods 91:155-163.

156. Klionsky DJ, Herman PK, Emr SD. 1990. The fungal vacuole: composition, function, and biogenesis. Microbiol Rev 54:266-292.

157. Veses V, Richards A, Gow NA. 2008. Vacuoles and fungal biology. Curr Opin Microbiol 11:503-510.

158. Finbow ME, Harrison MA. 1997. The vacuolar H+-ATPase: a universal proton pump of eukaryotes. Biochem J 324:697-712.

159. Cyert MS, Philpott CC. 2013. Regulation of cation balance in Saccharomyces cerevisiae. Genetics 193:677-713.

160. Pittman JK. 2011. Vacuolar Ca2+ uptake. Cell Calcium 50:139-146. 
161. Cunningham KW. 2011. Acidic calcium stores of Saccharomyces cerevisiae. Cell Calcium 50:129-138.

162. Cagnac O, Aranda-Sicilia MN, Leterrier M, Rodriguez-Rosales MP, Venema K. 2010. Vacuolar cation/H+ antiporters of Saccharomyces cerevisiae. J Biol Chem 285:33914-33922.

163. Ramsay LM, Gadd GM. 1997. Mutants in Saccharomyces cerevisiae defective in vacuolar function confirm a role for the vacuole in toxic metal ion detoxification. FEMS Microbiol Lett 152:296-298.

164. Li Z-S, Lu Y-P, Zhen R-G, Szczypka M, Thiele DJ, Rea PA. 1997. A new pathway for vacuolar cadmium sequestration in Saccharomyces cerevisiae: YCF1-catalyzed transport of bis(glutathionato)cadmium. Proc Natl Acad Sci U S A 94:42-47.

165. Bode H-P, Dumschat M, Garotti S, Fuhrmann GF. 1995. Iron Sequestration by the Yeast Vacuole. Eur J Biochem 228:337-342.

166. Palmer GE, Kelly MN, Sturtevant JE. 2005. The Candida albicans vacuole is required for differentiation and efficient macrophage killing. Eukaryot Cell 4:1677-1686.

167. Veses V, Richards A, Gow NA. 2009. Vacuole inheritance regulates cell size and branching frequency of Candida albicans hyphae. Mol Microbiol 71:505-519.

168. Veses V, Gow NA. 2008. Vacuolar dynamics during the morphogenetic transition in Candida albicans. FEMS Yeast Res 8:1339-1348.

169. Heese-Peck A, Pichler H, Zanolari B, Watanabe R, Daum G, Riezman H. 2002. Multiple functions of sterols in yeast endocytosis. Mol Biol Cell 13:26642680.

170. Kato M, Wickner WT. 2001. Ergosterol is required for the Sec18/ATPdependent priming step of homotypic vacuole fusion. EMBO J 20:4035-4040.

171. Seeley ES, Kato M, Margolis N, Wickner W, Eitzen G. 2002. Genomic analysis of homotypic vacuole fusion. Mol Biol Cell 13:782-794.

172. Luna-Tapia A, Peters BM, Eberle KE, Kerns ME, Foster TP, Marrero L, Noverr MC, Fidel PL, Jr., Palmer GE. 2015. ERG2 and ERG24 Are Required for Normal Vacuolar Physiology as Well as Candida albicans Pathogenicity in a Murine Model of Disseminated but Not Vaginal Candidiasis. Eukaryot Cell 14:1006-1016.

173. Zinser E, Paltauf E, Daum G. 1993. Sterol composition of yeast organelle membranes and subcellular distribution of enzymes involved in sterol metabolism. J Bacteriol 175:2853-2858.

174. Estrada AF, Muruganandam G, Prescianotto-Baschong C, Spang A. 2015. The ArfGAP2/3 Glo3 and ergosterol collaborate in transport of a subset of cargoes. Biol Open 4:792-802.

175. Schmidt CL, Grey M, Schmidt M, Brendel M, Henriques JAP. 1999. Allelism of Saccharomyces cerevisiae genes PSO6, involved in survival after 3-CPs+UVA induced damage, and $E R G 3$, encoding the enzyme sterol C-5 desaturase. Yeast 15:1503-1510.

176. Pesti M, Campbell JM, Peberdy JF. 1981. Alteration of ergosterol content and chitin synthase activity in Candida albicans. Curr Microbiol 5:187-190. 
177. Bagnat M, Keranen S, Shevchenko A, Shevchenko A, Simons K. 2000. Lipid rafts function in biosynthetic delivery of proteins to the cell surface in yeast. Proc Natl Acad Sci U S A 97:3254-3259.

178. Jin H, McCaffery JM, Grote E. 2008. Ergosterol promotes pheromone signaling and plasma membrane fusion in mating yeast. J Cell Biol 180:813-826.

179. Deng L, Nagasawa J, Ono Y, Ishikawa Y, Kakihara T, Fukuda R, Ohta A. 2008. Manipulation of major membrane lipid synthesis and its effects on sporulation in Saccharomyces cerevisiae. Biosci Biotechnol Biochem 72:23622368.

180. Ha KC, White TC. 1999. Effects of Azole Antifungal Drugs on the Transition from Yeast Cells to Hyphae in Susceptible and Resistant Isolates of the Pathogenic Yeast Candida albicans. Antimicrob Agent Chemother 43:763-768.

181. Kenna S, Bligh HFJ, Watson PF, Kelly SL. 1989. Genetic and physiological analysis of azole sensitivity in Saccharomyces cerevisiae. J Med Vet Mycol 27:397-406.

182. Palmer GE. 2011. Vacuolar trafficking and Candida albicans pathogenesis. Commun Integr Biol 4:1-3.

183. Patenaude C, Zhang Y, Cormack B, Kohler J, Rao R. 2013. Essential Role for Vacuolar Acidification in Candida albicans Virulence. J Biol Chem 288:2625626264.

184. Raines SM, Rane HS, Bernardo SM, Binder JL, Lee SA, Parra KJ. 2013. Deletion of vacuolar proton-translocating ATPase V(o)a isoforms clarifies the role of vacuolar $\mathrm{pH}$ as a determinant of virulence-associated traits in Candida albicans. J Biol Chem 288:6190-6201.

185. Burke D, Dawson D, Stearns T, Laboratory CSH. 2000. Methods in Yeast Genetics: A Cold Spring Harbor Laboratory Course Manual. Cold Spring Harbor Laboratory Press.

186. Luna-Tapia A, Kerns ME, Eberle KE, Jursic BS, Palmer GE. 2015. Trafficking through the Late Endosome Significantly Impacts Candida albicans Tolerance of the Azole Antifungals. Antimicrob Agent Chemother 59:2410-2420.

187. Johnston DA, Luna-Tapia A, Eberle KE, Palmer GE. 2013. Three prevacuolar compartment Rab GTPases impact Candida albicans hyphal growth. Eukaryot Cell 12:1039-1050.

188. Mukhtar M, Logan DA, Kaufer NF. 1992. The carboxypeptidase Y-encoding gene from Candida albicans and its transcription during yeast-to-hyphae conversion. Gene 121:173-177.

189. Gerami-Nejad M, Berman J, Gale CA. 2001. Cassettes for PCR-mediated construction of green, yellow, and cyan fluorescent protein fusions in Candida albicans. Yeast 18:859-864.

190. Wilson RB, Davis D, Mitchell AP. 1996. Rapid hypothesis testing with Candida albicans through gene disruption with short homology regions. J Bacteriol 181:1868 - 1874 .

191. Nakayama H, Mio T, Nagahashi S, Kokado M, Arisawa M, Aoki Y. 2000. Tetracycline-regulatable system to tightly control gene expression in the pathogenic fungus Candida albicans. Infect Immun 68:6712-6719. 
192. Boeke JD, La Croute F, Fink GR. 1984. A positive selection for mutants lacking orotidine-5'-phosphate decarboxylase activity in yeast: 5-fluoro-orotic acid resistance. Mol Gen Genet 197:345-346.

193. Fonzi WA, Irwin MY. 1993. Isogenic strain construction and gene mapping in Candida albicans. Genetics 134:717-728.

194. Cope JE. 1980. Mode of Action of Miconazole on Candida albicans: Effect on Growth, Viability and K+ release. J Gen Microbiol 119:245-251.

195. Thevissen K, Ayscough KR, Aerts AM, Du W, De Brucker K, Meert EM, Ausma J, Borgers M, Cammue BP, Francois IE. 2007. Miconazole induces changes in actin cytoskeleton prior to reactive oxygen species induction in yeast. $\mathrm{J}$ Biol Chem 282:21592-21597.

196. François IEJA, Cammue BPA, Borgers M, Ausma J, Dispersyn GD, Thevissen K. 2006. Azoles: Mode of Antifungal Action and Resistance Development. Effect of Miconazole on Endogenous Reactive Oxygen Species Production in Candida albicans. Antiinfect Agents Med Chem 5:3-13.

197. Bacia K, Schwille P, Kurzchalia T. 2005. Sterol structure determines the separation of phases and the curvature of the liquid-ordered phase in model membranes. Proc Natl Acad Sci U S A 102:3272-3277.

198. Bayer MJ, Reese C, Buhler S, Peters C, Mayer A. 2003. Vacuole membrane fusion: V0 functions after trans-SNARE pairing and is coupled to the Ca2+releasing channel. J Cell Biol 162:211-222.

199. Baars TL, Petri S, Peters C, Mayer A. 2007. Role of the V-ATPase in regulation of the vacuolar fission-fusion equilibrium. Mol Biol Cell 18:38733882.

200. Palanisamy SK, Ramirez MA, Lorenz M, Lee SA. 2010. Candida albicans PEP12 is required for biofilm integrity and in vivo virulence. Eukaryot Cell 9:266-277.

201. Bowers K, Stevens TH. 2005. Protein transport from the late Golgi to the vacuole in the yeast Saccharomyces cerevisiae. Biochim Biophys Acta 1744:438454.

202. Becherer KA, Rieder SE, Emr SD, Jones EW. 1996. Novel syntaxin homologue, Pep12p, required for the sorting of lumenal hydrolases to the lysosome-like vacuole in yeast. Mol Biol Cell 7:579-594.

203. von Mollard GF, Stevens TH. 1999. The Saccharomyces cerevisiae v-SNARE Vtilp Is Required for Multiple Membrane Transport Pathways to the Vacuole. Mol Biol Cell 10:1719-1732.

204. Horazdovsky BF, Busch GR, Emr SD. 1994. VPS21 encodes a rab5-like GTP binding protein that is required for the sorting of yeast vacuolar proteins. EMBO J 13:1297-1309.

205. Hama H, Tall GG, Horazdovsky BF. 1999. Vps9p Is a Guanine Nucleotide Exchange Factor Involved in Vesicle-mediated Vacuolar Protein Transport. J Biol Chem 274:15284-15291.

206. Peterson MR, Burd CG, Emr SD. 1999. Vac1p coordinates Rab and phosphatidylinositol 3-kinase signaling in Vps45p-dependent vesicle docking/fusion at the endosome. Curr Biol 9:S1-162. 
207. Gerrard SR, Bryant NJ, Stevens TH. 2000. VPS21 Controls Entry of Endocytosed and Biosynthetic Proteins into the Yeast Prevacuolar Compartment. Mol Biol Cell 11:613-626.

208. Cebollero E, Reggiori F. 2009. Regulation of autophagy in yeast Saccharomyces cerevisiae. Biochim Biophys Acta 1793:1413-1421.

209. Mizushima N, Levine B. 2010. Autophagy in mammalian development and differentiation. Nat Cell Biol 12:823-830.

210. Noda T, Kim J, Huang W-P, Baba M, Tokunaga C, Ohsumi Y, Klionsky DJ. 2000. Apg9p/Cvt7p Is an Integral Membrane Protein Required for Transport Vesicle Formation in the Cvt and Autophagy Pathways. J Cell Biol 148:465-480.

211. Wickner W. 2010. Membrane fusion: five lipids, four SNAREs, three chaperones, two nucleotides, and a Rab, all dancing in a ring on yeast vacuoles. Annu Rev Cell Dev Biol 26:115-136.

212. Noda T, Fujita N, Yoshimori T. 2009. The late stages of autophagy: how does the end begin? Cell Death Differ 16:984-990.

213. Ramon AM, Fonzi WA. 2003. Diverged Binding Specificity of Rim101p, the Candida albicans Ortholog of PacC. Eukaryot Cell 2:718-728.

214. Palmer GE, Kelly MN, Sturtevant JE. 2007. Autophagy in the pathogen Candida albicans. Microbiology 153:51-58.

215. Bensen ES, Filler SG, Berman J. 2002. A Forkhead Transcription Factor Is Important for True Hyphal as well as Yeast Morphogenesis in Candida albicans. Eukaryot Cell 1:787-798.

216. Gillum AM, Tsay EY, Kirsch DR. 1984. Isolation of the Candida albicans gene for orotidine-5'-phosphate decarboxylase by complementation of $S$. cerevisiae ura3 and E. coli pyrF mutations. Molecular and General Genetics 198:179-182.

217. Gietz D, St. Jean A, Woods RA, Schiestl RH. 1992. Improved method for high efficiency transformation of intact yeast cells. Nucleic Acids Res 20:1425.

218. CLSI. 2008. Reference Method for Broth Dilution Antifungal Suceptibility Testing of Yeasts; Approved Standar-Third Edition. CLSI document M27-A3. Clinical and Laboratory Standards Institute, Wayne, PA.

219. Arthington-Skaggs BA, Jradi H, Desai T, Morrison CJ. 1999. Quantitation of ergosterol content novel method for determination of fuconazole susceptibility of Candida albicans. J Clin Microbiol 37:3332-3337.

220. Xu W, Smith FJ, Jr., Subaran R, Mitchell AP. 2004. Multivesicular bodyESCRT components function in $\mathrm{pH}$ response regulation in Saccharomyces cerevisiae and Candida albicans. Mol Biol Cell 15:5528-5537.

221. Agrawal D, Patterson TF, Rinaldi MG, Revankar SG. 2007. Trailing endpoint phenotype of Candida spp. in antifungal susceptibility testing to fluconazole is eliminated by altering incubation temperature. J Med Microbiol 56:1003-1004.

222. Liu Y, Solis NV, Heilmann CJ, Phan QT, Mitchell AP, Klis FM, Filler SG. 2014. Role of retrograde trafficking in stress response, host cell interactions, and virulence of Candida albicans. Eukaryot Cell 13:279-287.

223. Cornet M, Gaillardin C, Richard ML. 2006. Deletions of the endocytic components VPS28 and VPS32 in Candida albicans lead to echinocandin and azole hypersensitivity. Antimicrob Agent Chemother 50:3492-3495. 
224. Prinz WA. 2007. Non-vesicular sterol transport in cells. Prog Lipid Res 46:297314.

225. Baumann NA, Sullivan DJ, Ohvo-Rekila H, Simonot C, Pottekat A, Klaassen Z, Beh CT, Menon AK. 2005. Transport of newly synthesized sterol to the sterol-enriched plasma membrane occurs via nonvesicular equilibration. Biochemistry 44:5816-5826.

226. Schulz TA, Prinz WA. 2007. Sterol transport in yeast and the oxysterol binding protein homologue (OSH) family. Biochim Biophys Acta 1771:769-780.

227. Smith WL, Edlind TD. 2002. Histone Deacetylase Inhibitors Enhance Candida albicans Sensitivity to Azoles and Related Antifungals: Correlation with Reduction in $C D R$ and $E R G$ Upregulation. Antimicrob Agent Chemother 46:3532-3539.

228. Luna-Tapia A, Tournu H, Peters TL, Palmer GE. 2016. Endosomal trafficking defects can induce calcium dependent azole tolerance in Candida albicans. Antimicrob Agent Chemother 60:00-00.

229. Gillum AM, Tsay EYH, Kirsch DR. 1984. Isolation of the Candida albicans gene for orotidine-5'-phosphate decarboxylase by complementation of $S$. cerevisiae ura3 and E. coli pyrF mutations. Mol Gen Genet 198:179-182.

230. Wilson RB, Davis D, Enloe BM, Mitchell AP. 2000. A recyclable Candida albicans URA3 cassette for PCR product-directed gene disruptions. Yeast 16:6570.

231. Hall MP, Unch J, Binkowski BF, Valley MP, Butler BL, Wood MG, Otto P, Zimmerman K, Vidugiris G, Machleidt T, Robers MB, Benink HA, Eggers CT, Slater MR, Meisenheimer PL, Klaubert DH, Fan F, Encell LP, Wood KV. 2012. Engineered luciferase reporter from a deep sea shrimp utilizing a novel imidazopyrazinone substrate. ACS Chemical Biology 7:1848-1857.

232. Kmit A, van Kruchten R, Ousingsawat J, Mattheij NJ, Senden-Gijsbers B, Heemskerk JW, Schreiber R, Bevers EM, Kunzelmann K. 2013. Calciumactivated and apoptotic phospholipid scrambling induced by Ano6 can occur independently of Ano6 ion currents. Cell Death Dis 4:e611.

233. Baggett JJ, Shaw JD, Sciambi CJ, Watson HA, Wendland B. 2001. Fluorescent Labeling of Yeast, Current Protocols in Cell Biology doi:10.1002/0471143030.cb0413s20. John Wiley \& Sons, Inc.

234. Sambade M, Alba M, Smardon AM, West RW, Kane PM. 2005. A genomic screen for yeast vacuolar membrane ATPase mutants. Genetics 170:1539-1551.

235. Zhang C, Konopka JB. 2010. A photostable green fluorescent protein variant for analysis of protein localization in Candida albicans. Eukaryot Cell 9:224-226.

236. Gerrard SR, Levi BP, Stevens TH. 2000. Pep12p is a Multifunctional Yeast Syntaxin that Controls Entry of Biosynthetic, Endocytic and Retrograde Traffic into the Prevacuolar Compartment. Traffic 1:259-269.

237. Onyewu C, Wormley FL, Perfect JR, Heitman J. 2004. The Calcineurin Target, Crz1, Functions in Azole Tolerance but Is Not Required for Virulence of Candida albicans. Infect Immun 72:7330-7333.

238. Kaur R, Castano I, Cormack BP. 2004. Functional Genomic Analysis of Fluconazole Susceptibility in the Pathogenic Yeast Candida glabrata: Roles of 
Calcium Signaling and Mitochondria. Antimicrob Agent Chemother 48:16001613.

239. Gupta SS, Ton VK, Beaudry V, Rulli S, Cunningham K, Rao R. 2003. Antifungal activity of amiodarone is mediated by disruption of calcium homeostasis. J Biol Chem 278:28831-28839.

240. Paidhungat M, Garrett S. 1997. A homolog of mammalian, voltage-gated calcium channels mediates yeast pheromone-stimulated $\mathrm{Ca} 2+$ uptake and exacerbates the cdc1(Ts) growth defect. Mol Cell Biol 17:6339-6347.

241. Uppuluri P, Nett J, Heitman J, Andes D. 2008. Synergistic effect of calcineurin inhibitors and fluconazole against Candida albicans biofilms. Antimicrob Agent Chemother 52:1127-1132.

242. Jia Y, Tang RJ, Wang L, Zhang X, Wang Y, Jia XM, Jiang YY. 2012. Calcium-activated-calcineurin reduces the In vitro and In vivo sensitivity of fluconazole to Candida albicans via Rta2p. PLoS One 7:e48369.

243. Jia XM, Wang Y, Jia Y, Gao PH, Xu YG, Wang L, Cao YY, Cao YB, Zhang LX, Jiang YY. 2009. RTA2 is involved in calcineurin-mediated azole resistance and sphingoid long-chain base release in Candida albicans. Cell Mol Life Sci 66:122-134.

244. Thomas E, Sircaik S, Roman E, Brunel J-M, Johri AK, Pla J, Panwar SL. 2015. The activity of RTA2, a downstream effector of the calcineurin pathway, is required during tunicamycin-induced ER stress response in Candida albicans. FEMS Yeast Res 15:fov095.

245. Barker KS, Crisp S, Wiederhold N, Lewis RE, Bareither B, Eckstein J, Barbuch R, Bard M, Rogers PD. 2004. Genome-wide expression profiling reveals genes associated with amphotericin B and fluconazole resistance in experimentally induced antifungal resistant isolates of Candida albicans. $\mathbf{J}$ Antimicrob Chemother 54:376-385.

246. Peters BM, Palmer GE, Nash AK, Lilly EA, Fidel PL, Noverr MC. 2014. Fungal Morphogenetic Pathways Are Required for the Hallmark Inflammatory Response during Candida albicans Vaginitis. Infect Immun 82:532-543.

247. Schnabl M, Daum G, Pichler H. 2005. Multiple lipid transport pathways to the plasma membrane in yeast. Biochim Biophys Acta 1687:130-140.

248. Beckers CJ, Balch WE. 1989. Calcium and GTP: essential components in vesicular trafficking between the endoplasmic reticulum and Golgi apparatus. J Cell Biol 108:1245-1256.

249. Colombo MI, Beron W, Stahl PD. 1997. Calmodulin Regulates Endosome Fusion. J Biol Chem 272:7707-7712.

250. Peters C, Mayer A. 1998. Ca2+/calmodulin signals the completion of docking and triggers a late step of vacuole fusion. Nature 396:575-580.

251. Antebi A, Fink GR. 1992. The yeast $\mathrm{Ca}(2+)$-ATPase homologue, PMR1, is required for normal Golgi function and localizes in a novel Golgi-like distribution. Mol Biol Cell 3:633-654.

252. Locke EG, Bonilla M, Liang L, Takita Y, Cunningham KW. 2000. A Homolog of Voltage-Gated $\mathrm{Ca}^{2+}$ Channels Stimulated by Depletion of Secretory $\mathrm{Ca}^{2+}$ in Yeast. Mol Cell Biol 20:6686-6694. 


\section{APPENDIX A. SUPPLEMENTAL FIGURES FOR CHAPTER 3}

Figure A-1. The C. albicans Vps21p paralogs Ypt52p and Ypt53p do not significantly affect susceptibility to miconazole.

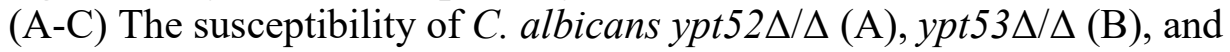
$y p t 52 \Delta / \Delta y p t 53 \Delta / \Delta(\mathrm{C})$ mutants to miconazole was tested using the standard CLSI broth microdilution protocol. After 48 hours incubation, growth was measured as $\mathrm{OD}_{600 \mathrm{~nm}}$ and expressed as a percentage of the growth in the minus drug (DMSO alone) control wells. (D-F) The susceptibility of C. albicans ypt52 $\Delta / \Delta y p t 53 \Delta / \Delta$ (D), vps $21 \Delta / \Delta y p t 52 \Delta / \Delta$ (E) and vps $21 \Delta / \Delta y p t 53 \Delta / \Delta$ (F) double mutants was tested as described above. The mean of at least two independent experimental replicates is shown for all panels. 
A.

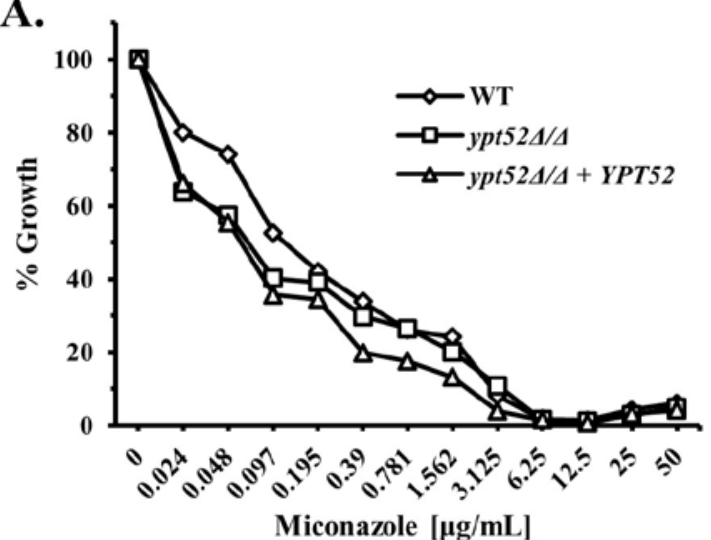

C.

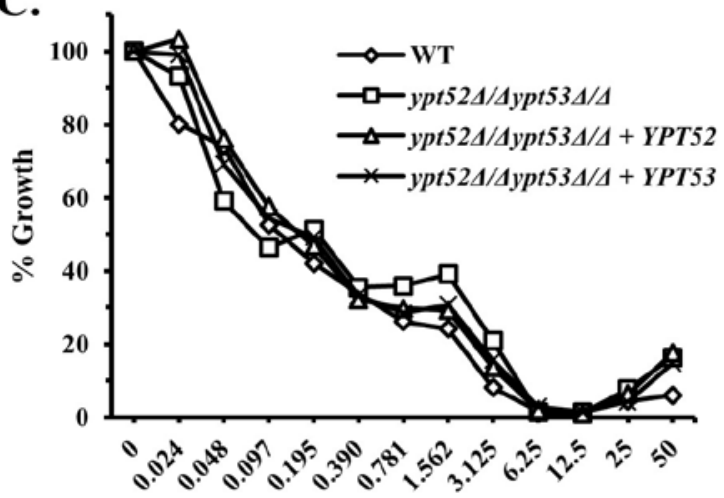

Miconazole $[\mu \mathrm{g} / \mathrm{mL}]$
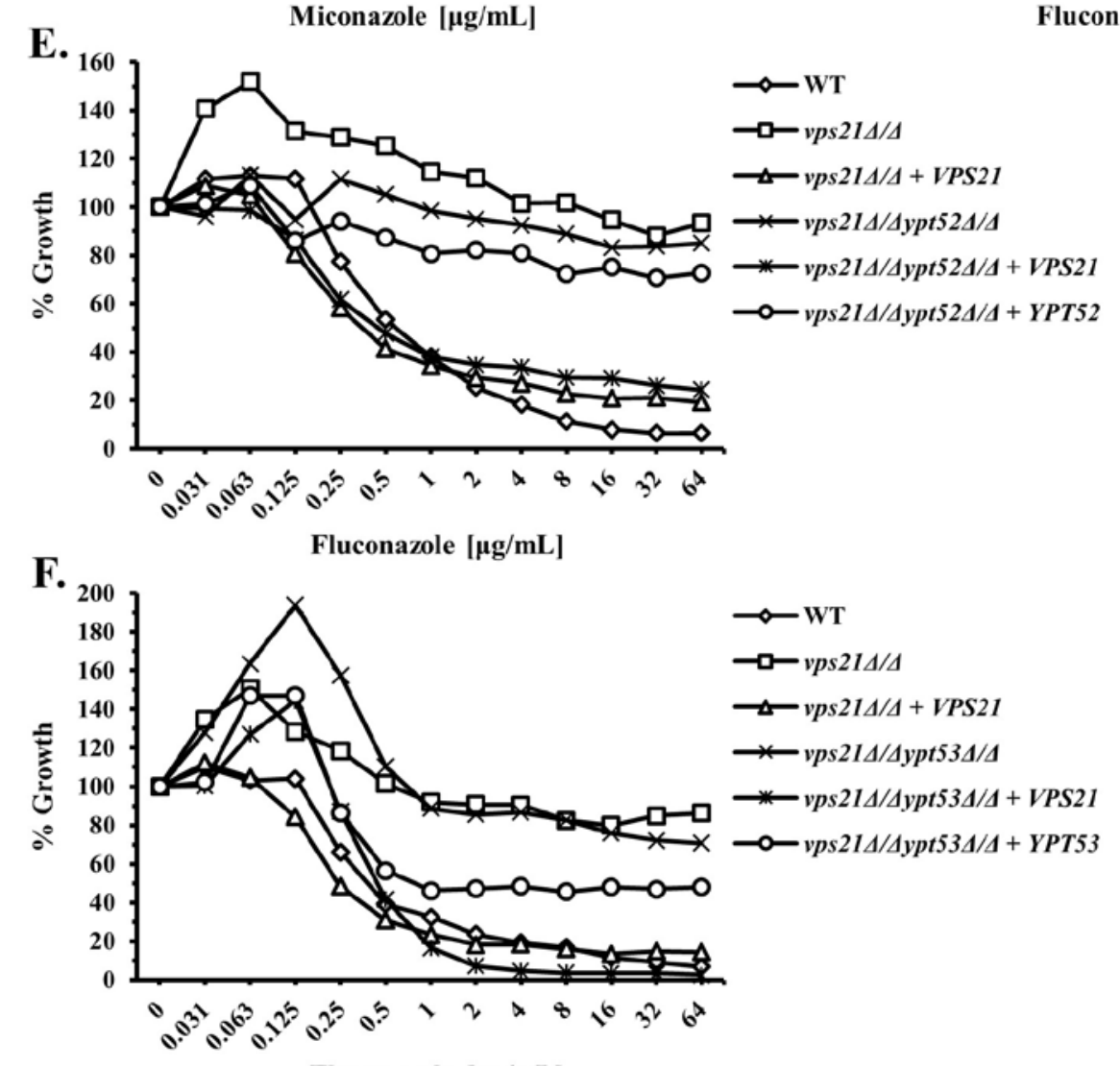

Fluconazole $[\mu \mathrm{g} / \mathrm{mL}]$

\footnotetext{
$\infty$ wT
}

$\rightarrow-v p s 214 / 4$

$\longrightarrow v p s 214 / 4+V P S 21$

— $v p s 214 / 4 y p t 524 / 4$

* vps214/4ypt524/4 + VPS21
$\multimap-v p s 214 / 4 y p t 524 / 4+Y P T 52$

$\multimap$ - vps214/Aypt524/A + YPT52

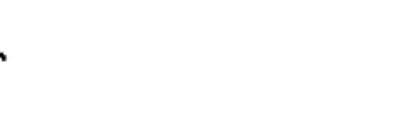

B.

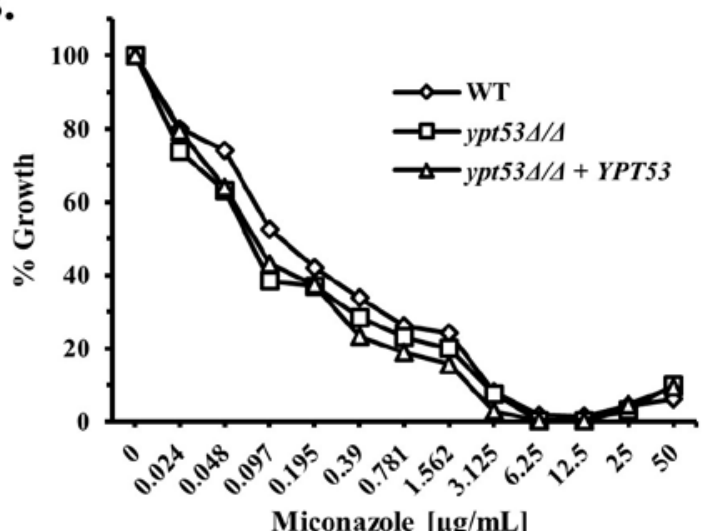

D.

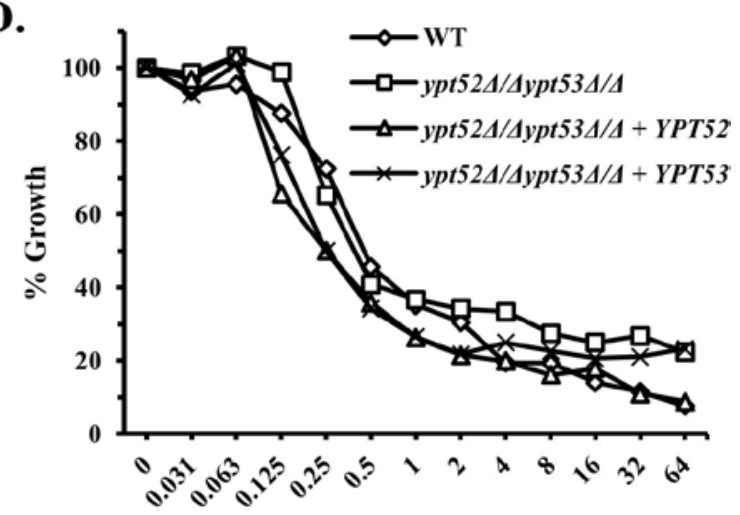

Fluconazole $[\mu \mathrm{g} / \mathrm{mL}]$

Cluconate $\{$ g 


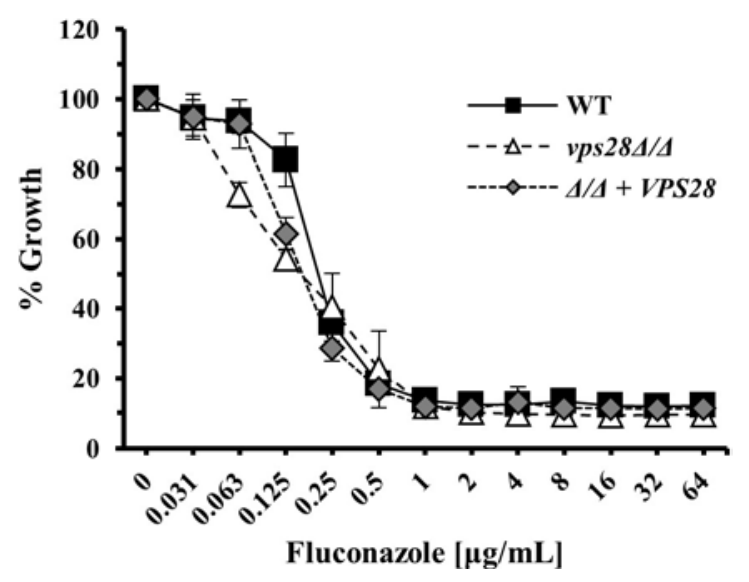

Figure A-2. Deletion of VPS28 does not affect $C$. albicans susceptibility to fluconazole.

The susceptibility of the $C$. albicans vps $28 \Delta / \Delta$ mutant and control strains to fluconazole was evaluated using the standard CLSI broth microdilution protocol. After 48 hours incubation, growth was measured as $\mathrm{OD}_{600 \mathrm{~nm}}$ and expressed as a percentage of the growth in the minus drug (DMSO alone) control wells. The means \pm standard deviations of three biological replicates are shown. 
A.

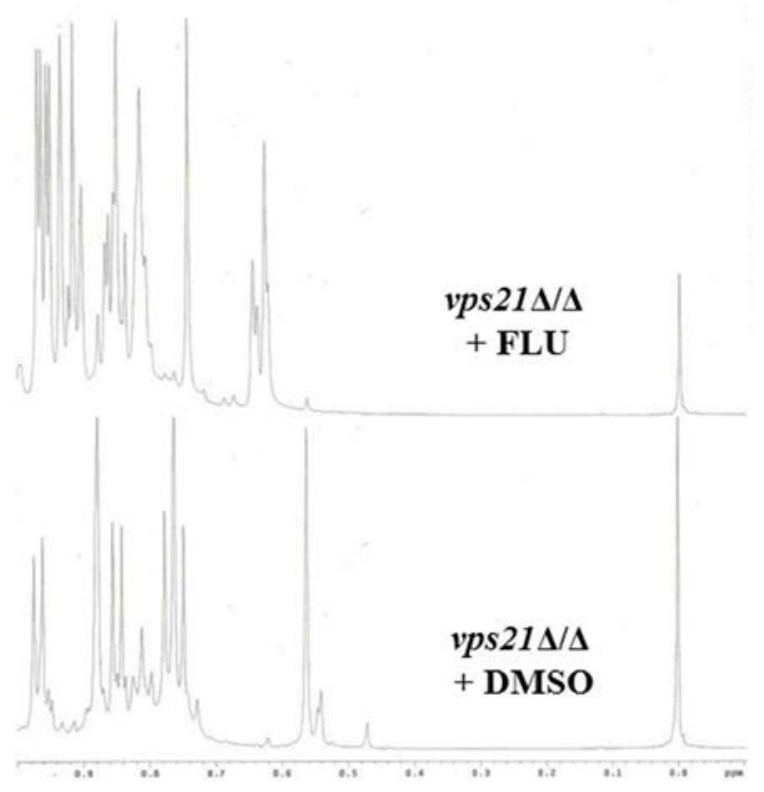

B.

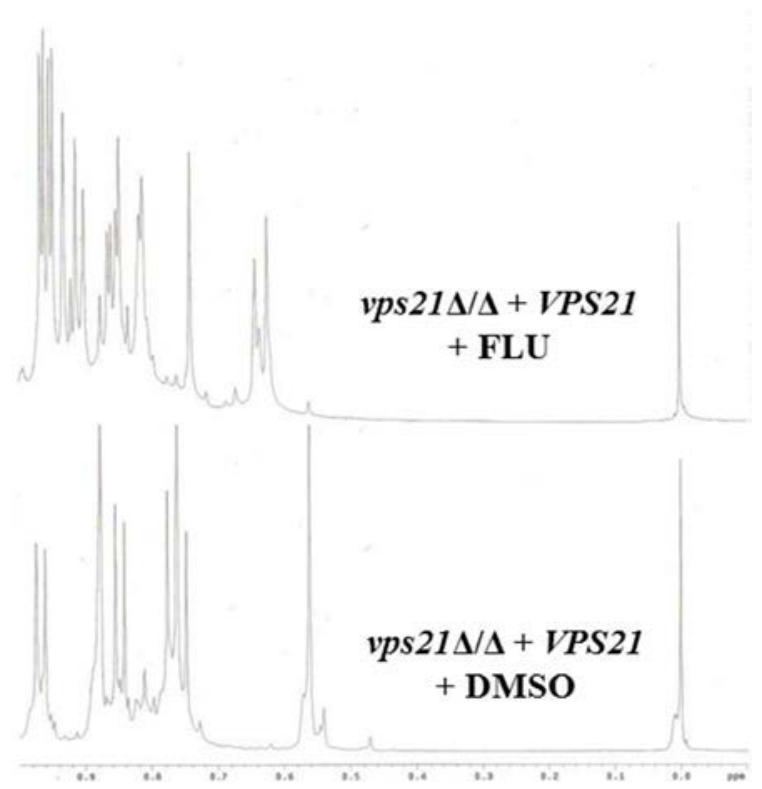

Figure A-3. The composition of sterols extracted from fluconazole-treated $v p s 21 \Delta / \Delta$ mutant is similar to that of the isogenic control.

The $C$. albicans vps $21 \Delta / \Delta$ mutant and its isogenic control strain were grown in YNB broth with $4 \mu \mathrm{g} / \mathrm{mL}$ fluconazole (A and B top panel) or 0.5\% DMSO (A and B bottom panel) for 24 hours at $35^{\circ} \mathrm{C}$, total cellular sterols extracted using n-heptane and analyzed by NMR analysis. 


\section{APPENDIX B. SUPPLEMENTAL FIGURES FOR CHAPTER 4}

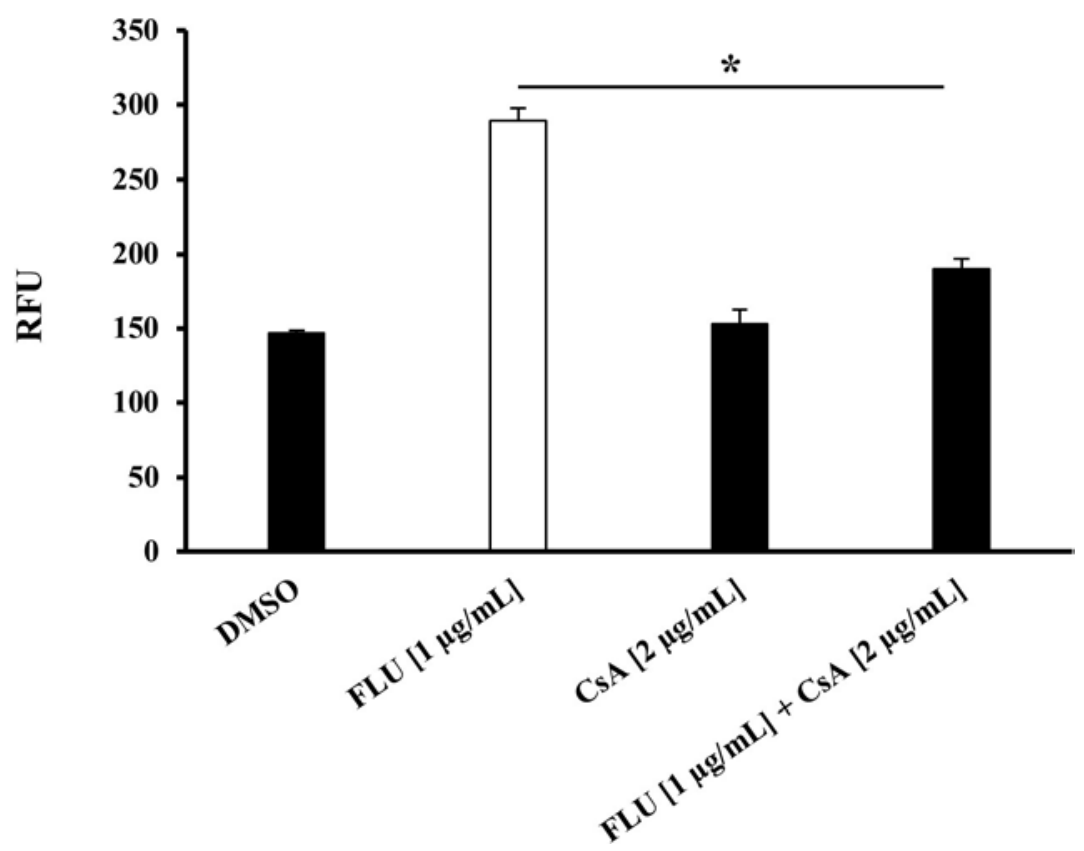

Figure B-1. Fluconazole induced expression of GFP from the RTA2 promoter is calcineurin dependent.

The RTA2pr-GFP $\gamma$ expression construct was introduced into C. albicans strain CAI4, and the resulting strain cultured in YNB medium with either $1 \mu \mathrm{g} / \mathrm{mL}$ fluconazole (FLU), 2 $\mu \mathrm{g} / \mathrm{mL}$ cyclosporine $\mathrm{A}(\mathrm{CsA})$, a combination of both drugs, or $0.5 \%$ DMSO. Cells were incubated for 24 hours at $30^{\circ} \mathrm{C}$. Fluorescence intensity was determined by excitation at $488 \mathrm{~nm}$ and emission at $507 \mathrm{~nm}$, normalized for cell density $\left(\mathrm{OD}_{600 \mathrm{~nm}}\right)$, and expressed as relative fluorescence units (RFU). The mean \pm standard deviation of two biological replicates is shown. A two-tailed $t$-test was used to compare the mean of each group; * $P$ $<0.006$. 
A.

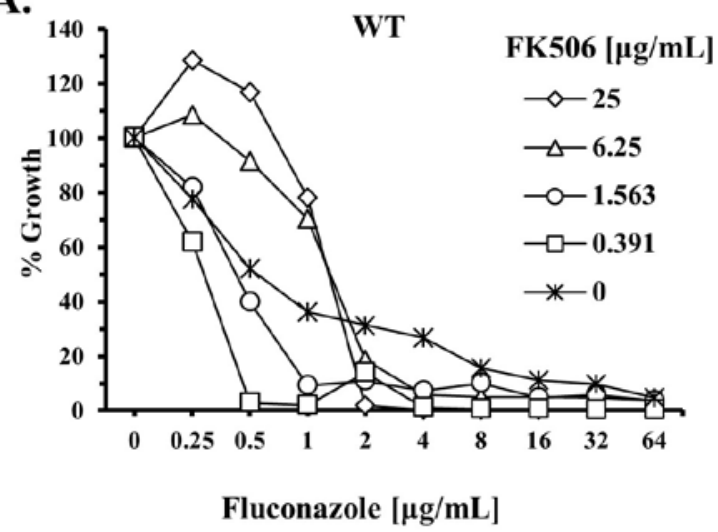

B.

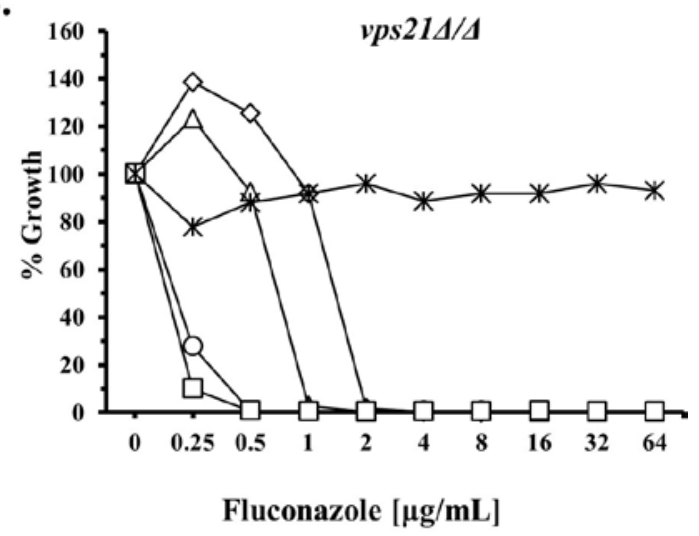

\section{C.}

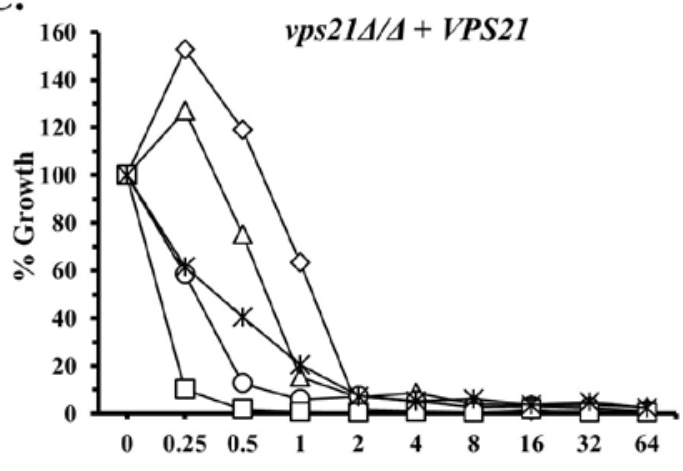

Fluconazole $[\mu \mathrm{g} / \mathrm{mL}]$

Figure B-2. The calcineurin signaling inhibitor FK506 eliminates the $C$. albicans $v p s 21 \Delta / \Delta$ mutant's azole tolerance.

The effect of calcineurin signaling inhibition with FK506 on azole tolerance in the WT (A), vps $21 \Delta / \Delta$ mutant (B) and isogenic control strain (C) was evaluated using a checkerboard assay using the standard CLSI broth microdilution protocol. Growth was determined as a percentage of the minus drug (DMSO) control. A representative experiment is shown, but similar results were obtained in a second repeat experiment. 
APPENDIX C. LIST OF STRAINS DESCRIBED IN CHAPTER 6

\begin{tabular}{|c|c|c|}
\hline Strain & Relevant Genotype & Reference \\
\hline YJB6284 & ura3 $\Delta / \Delta: U R A 3$ his $1 \Delta / \Delta: H I S 1$ arg $4 \Delta / \Delta: A R G 4$ & $(215)$ \\
\hline $\operatorname{vps} 21 \Delta / \Delta$ & 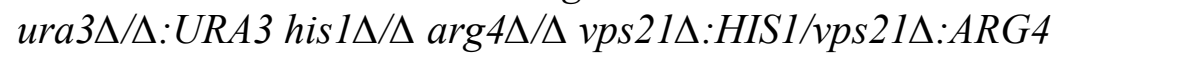 & $(51)$ \\
\hline$v p s 21 \Delta / \Delta+V P S 21$ & ura $3 \Delta / \Delta: U R A 3: V P S 21$ his $1 \Delta / \Delta \arg 4 \Delta / \Delta$ vps $21 \Delta: H I S 1 / v p s 21 \Delta: A R G 4$ & $(51)$ \\
\hline $\operatorname{aps} 3 \Delta / \Delta$ & ura $3 \Delta / \Delta: U R A 3$ his $1 \Delta / \Delta \arg 4 \Delta / \Delta$ aps $3 \Delta: H I S 1 / a p s 3 \Delta: A R G 4$ & $(50)$ \\
\hline aps $3 \Delta / \Delta+A P S 3$ & ura $3 \Delta / \Delta: U R A 3: A P S 3$ his $1 \triangle / \Delta \arg 4 \Delta / \triangle$ aps $3 \Delta: H I S 1 / a p s 3 \Delta: A R G 4$ & $(50)$ \\
\hline $\operatorname{vps} 21 \Delta / \Delta \operatorname{aps} 3 \Delta / \Delta$ & $\begin{array}{l}\text { ura3 } 3 / \Delta: U R A 3 \text { his } 1 \Delta / \Delta \arg 4 \Delta / \Delta \text { vps } 21 \Delta: H I S 1 / v p s 21 \Delta: d p l 200 \\
\text { aps } 3 \Delta: A R G 4 / a p s 3 \Delta: d p l 200\end{array}$ & $(50)$ \\
\hline vps $21 \Delta / \Delta a p s 3 \Delta / \Delta+V P S 21$ & $\begin{array}{l}\text { ura } 3 \Delta / \Delta: U R A 3: V P S 21 \text { his } 1 \Delta / \Delta \text { arg } 4 \Delta / \Delta \text { vps } 21 \Delta: H I S 1 / v p s 21 \Delta: d p l 200 \\
\text { aps } 3 \Delta: A R G 4 / \text { aps } 3 \Delta: d p l 200\end{array}$ & $(50)$ \\
\hline vps $21 \Delta / \triangle a p s 3 \Delta / \Delta+A P S 3$ & 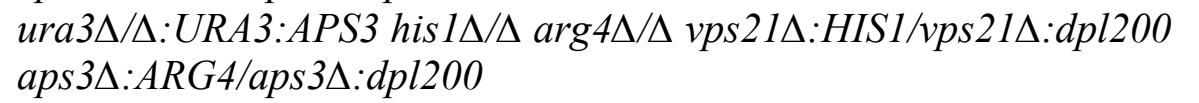 & $(50)$ \\
\hline$v c x 1 \Delta / \Delta$ & ura3 $\Delta / \Delta: U R A 3$ his $1 \Delta / \Delta \arg 4 \Delta / \Delta v c x 1 \Delta: H I S 1 / v c x 1 \Delta: A R G 4$ & This study \\
\hline$v c x 1 \Delta / \Delta+V C X 1$ & ura3 $\Delta / \Delta: U R A 3: V C X 1$ his $1 \Delta / \Delta \arg 4 \Delta / \Delta$ vex $1 \Delta: H I S 1 / v c x 1 \Delta: A R G 4$ & This study \\
\hline$p m c 1 \Delta / \Delta$ & 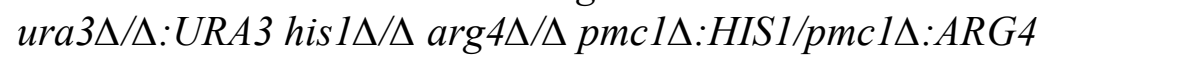 & This study \\
\hline$p m c 1 \Delta / \Delta+P M C 1$ & 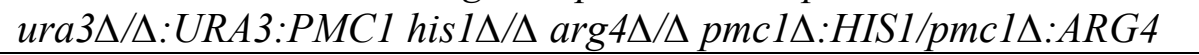 & This study \\
\hline
\end{tabular}




\section{VITA}

Arturo L. Luna, son of Luis Luna and Virgilia Tapia, was born in the town of Flor del Monte, Department of Sucre - Colombia in 1984. After graduating from high school at his hometown, he moved to the Department's capital Sincelejo to pursue college education, earning a Bsc in Biology in 2008 from the Universidad de Sucre. In 2010, he was awarded a Fulbright scholarship to pursue Graduate education in the United States. He entered the Microbiology, Immunology and Parasitology Graduate Program at LSU Health Sciences Center New Orleans in the Fall semester of 2011. In 2015 he moved with his advisor to Memphis, and transferred to The University of Tennessee Health Science Center to continue his graduate education in the Microbiology, Immunology and Biochemistry track of the Integrated Program in Biomedical Sciences. He expects to complete his doctoral degree by December 2016. 